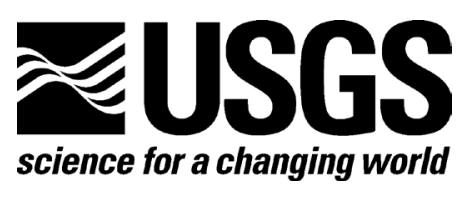

\title{
HAWAIIAN VOLCANO OBSERVATORY 1978 Annual Administrative Report
}

INTRODUCTORY NOTE BY THOMAS L. WRIGHT AND JENNIFER S. NAKATA

COMPILED BY JENNIFER S. NAKATA

SUMMARY 78, PART 1

SEISMIC DATA, JANUARY TO DECEMBER 1978

By Fred W. KLein, JenNifer S. NAKAta, And Robert Y. Koyanagi

CHRONOLOGICAL SUMMARY

BY RICHARD B. MOORE

OPEN-FILE REPORT 2007-1338

U.S. DEPARTMENT OF THE INTERIOR

U.S. GEOLOGICAL SURVEY 


\section{U.S. Department of the Interior DIRK KEMPTHORNE, Secretary \\ U.S. Geological Survey Mark D. Myers, Director}

\section{U.S. Geological Survey, Reston, Virginia 2007}

For product and ordering information:

World Wide Web: http://www.usgs.gov/pubprod

Telephone: 1-888-ASK-USGS

For more information on the USGS - the Federal source for science about the Earth, its natural and living resources, natural hazards, and the environment: World Wide Web: http://www.usgs.gov

Telephone: 1-888-ASK-USGS

Any use of trade, product, or firm names is for descriptive purposes only and does not imply endorsement by the U.S. Government.

Although this report is in the public domain, permission must be secured from the individual copyright owners to reproduce any copyrighted material contained within this report. 


\section{INTRODUCTORY NOTE}

The Hawaiian Volcano Observatory Summaries have been published in the current format since 1956. The Quarterly Summaries (1956 through 1973) and the Annual Summaries (1974 through 1985) were originally published as Administrative Reports. These reports have been compiled and published as U.S. Geological Survey Open-File Reports. The quarterly reports have been combined and published as one annual summary. All the summaries from 1956 to the present are now available as .pdf files at http://www.usgs.gov/pubprod.

The earthquake summary data are presented as a listing of origin time, depth, magnitude, and other location parameters. Network instrumentation, field station sites, and location algorithms are described. Tilt and other deformation data are included until Summary 77, January to December 1977. From 1978, the seismic and deformation data are published separately, due to differing schedules of data reduction.

There are eight quarters - from the fourth quarter of 1959 to the third quarter of 1961 — that were never published. Two of these ( $4^{\text {th }}$ quarter $1959,1^{\text {st }}$ quarter 1960) have now been published, using handwritten notes of Jerry Eaton (HVO seismologist at the time) and his colleagues. The seismic records for the remaining six summaries went back to California in 1961 with Jerry Eaton. Other responsibilities intervened, and the seismic summaries were never prepared.

\section{Chronology}

The following Kìlauea eruption chronology covers the two recent reports and the six missing quarters:

\begin{tabular}{|l|l|l|l|}
\hline Location & Beginning Date & Ending Date & Comment \\
\hline Kìlauea Iki crater (Kîlauea's summit) & $11 / 14 / 1959$ & $12 / 20 / 1959$ & 19 eruptive episodes \\
\hline Kapoho (lower east rift zone) & $1 / 13 / 1960$ & $2 / 18 / 1960$ & 4 eruption stages \\
\hline Halemaumau (Kīlauea's summit) & $2 / 24 / 1961$ & $2 / 24 / 1961$ & $\begin{array}{l}\text { Intermittent activity during } \\
\text { uninterrupted inflation fol- } \\
\text { lowing the 1960 eruption }\end{array}$ \\
\hline Halemaumau (Kīlauea's summit) & $3 / 22 / 1961$ & $3 / 25 / 1961$ & Same as above. \\
\hline Halemaumau (Kīlauea's summit) & $7 / 10 / 1961$ & $7 / 17 / 1961$ & Same as above. \\
\hline Heiheiahulu (middle east rift zone) & $9 / 22 / 1961$ & $9 / 25 / 1961$ & $\begin{array}{l}\text { First historical east rift erup- } \\
\text { tion at this location }\end{array}$ \\
\hline
\end{tabular}

The 1959-1960 eruptions were among two of the most spectacular Kilauea eruptions. The HVO staff was kept busy with acquisition of unusually high quantities of instrumental data and observations of the two sequences, which were separated by less than one month. Even with a year's interval before the beginning of the summit-east rift sequence in 1961, the staff never caught up, and the seismic records were set aside for later study.

A total of 1,672 earthquakes-1,106 for 1960 and 566 for 1961-are part of HVO's cataloged database. The annual listings have been appended to the $1^{\text {st }}$ Quarter Report of 1960 and to the $4^{\text {th }}$ Quarter Report for 1961 . The number of earthquakes is probably low, biased toward the larger magnitudes. The entire HVO catalog, including 1960 and 1961, is accessible from the ANSS CATALOG SEARCH site at http://www.ncedc.org/anss/catalog-search. 


\section{UNITED STATES DEPARTMENT OF THE INTERIOR GEOLOGICAL SURVEY}

HAWAIIAN VOLCANO OBSERVATORY SUMMARY 78, PART 1

SEISMIC DATA, JANUARY TO DECEMBER 1978

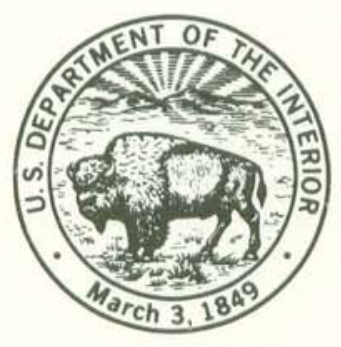

This report is preliminary and has not been edited or reviewed for conformity with Geological Survey standards and nomenclature Menlo Park, California 
UNITED STATES

DEPARTMENT OF THE INTERIOR

GEOLOGICAL SURVEY

HAWAIIAN VOLCANO OBSERVATORY

SUMMARY 78, PART 1

SEISMIC DATA, JANUARY TO DECEMBER 1978

BY

FRED W. KLEIN, JENNIFER S. NAKATA, ROBERT Y. KOYANAGI

CHRONOLOGICAL SUMMARY

BY

RICHARD B. MOORE

OBSERVATORY STAFF

\section{GEOLOGY}

JANE M. BUCHANAN-BANKS

NORMAN G. BANKS

DANIEL DZURISIN

GORDON P. EATON (SCIENTIST-IN-CHARGE)

JOHN P. LOCKWOOD

RICHARD B. MOORE

DONALD W. PETERSON

(ACTING SCIENTIST-IN-CHARGE)

\section{GEOPHYSICS}

LENNART A. ANDERSON

ROBERT K. CESSARO

KENNETH T. HONMA

DALLAS B. JACKSON

FRED W. KLEIN

GEORGE KOJ IMA

ROBERT Y. KOYANAGI

JENNIFER S. NAKATA

ARNOLD T. OKAMURA

GARY S. PUNIWAI

IRENE S. TAKAYESU

\section{GEOCHEMISTRY}

THOMAS J. CASADEVALL REGINALD T. OKAMURA

(CHIEF OF OPERATIONS)
SUPPORT

ALEX N. BITTENBINDER

THOMAS T. ENGLISH

JOHN C. FORBES

WILLIAM E. FRANCIS

JIM D. GRIGGS

JACK E. HARRIS

GAIL E. HILL

MARIE S. ONOUYE

MAURICE K. SAKO

AKIRA YAMAMOTO

KENNETH M. YAMASHITA 


\section{CONTENTS}

\section{Page}

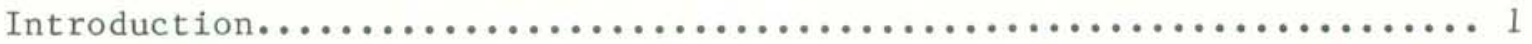

Chronological Summary..................................

Seismic Instrumentation.................................

Figure 1 Map of Hawaii showing geographic and geologic features................................4

Figure 2 Map of Hawaii showing active seismic stations and geographic regions........................ 5

Table 1 Seismic stations on Hawaii operated by the USGS....... 6

Table 2 Seismic instrumentation types in use by HVO......... 7

Figure 3 System response curve of the four basic seismograph types in use by HVO................... 8

Seismic Data Processing..................................

Figure 4 Relationship between duration time and local magnitude.................................

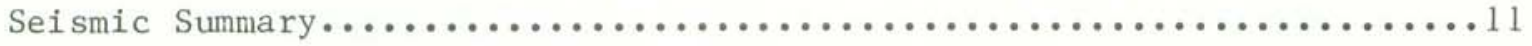

Table 3 Number of earthquakes and minutes of tremor recorded on seismographs around Kilauea.............. 12

Figure 5 Epicenter map of Hawaii showing only magnitudes

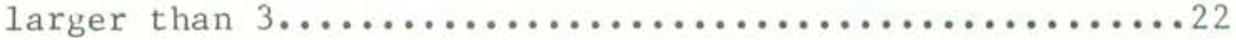

Figure 6 Epicenter map of Hawaii showing all earthquakes........23

Figure 7 Epicenter map of the Kilauea/Mauna Loa region showing only magnitudes larger than $3 \ldots \ldots \ldots \ldots \ldots \ldots \ldots \ldots$

Figure 8 Epicenter map of the Kilauea/Mauna Loa region

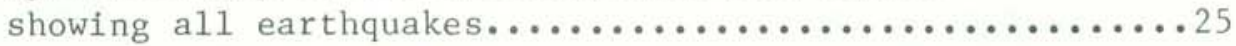

Figure 9 Epicenter map of Kilauea caldera.....................

Table 4 Coordinates of named regions used for classifying

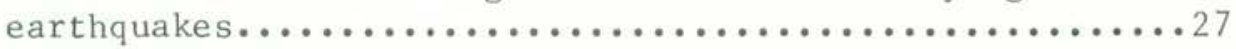

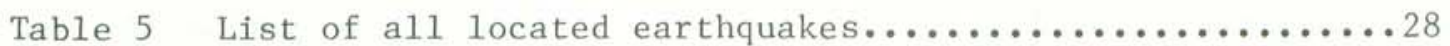

Table 6 List of located earthquakes of magnitude 3.0 or larger.................................... 54 


\section{INTRODUCTION}

The Hawaiian Volcano Observatory (HVO) summaries present data gathered during the year together with a chronological narrative intended to describe in geologic terms the volcanic activity associated with the seismic events and tilt data included. Beginning with this volume, the summaries will be issued in two parts. Seismic data will appear in Part 1, and water tube tilt and other deformation data will appear in Part 2. The seismic and chronological summaries are offered without interpretation as a source of preliminary data. The seismic summary is complete in the sense that all data routinely gathered by the observatory are included. The emphasis in collection of tilt and deformation data has recently shifted from quarterly measurements at a few water-tube tilt stations ("wet" tilt) to a larger number of continuously recording borehole tiltmeters, repeated measurements at numerous spirit-level tilt stations ("dry" tilt), and surveying of level and trilateration networks. Because of the large quantity of deformation data now gathered and differing schedules of data reduction, the seismic and deformation summaries will be published separately.

The HVO summaries have been published in various formats since 1956. Summaries prior to 1974 were issued quarterly, but cost, convenience of preparation and distribution, and the large quantities of data dictated an annual format beginning with summary 74 for the year 1974. Summary 74 includes an extensive description of the seismic instrumentation, calibration and processing used in recent years. The present summary includes enough background information on the seismic network and processing to use the data and understand the essentials of how it was gathered.

A report tabulating the instrumentation, calibration and recording history of each seismic station in the network is in preparation by Klein and Koyanagi as a USGS open file report. It is designed as a reference for users of seismograms and phase data, and so includes and goes a bit beyond the information in the station table in this summary. 
CHRONOLOGICAL SUMMARY - 1978

by

Richard B. Moore

Neither Kilauea nor Mauna Loa erupted during 1978, only the second such quiet year since 1966. Deflation of the southern summit area near Ahua associated with the September 1977 east rift eruption continued at a gradually diminishing rate until March 1978. A secondary center on the north side of the caldera, however, began to inflate in October 1977, shortly after the eruption ended. Both centers inflated during the rest of 1978, and by year's end about $75 \%$ of the level of inflation on September 12, 1977, just prior to the last eruption, had been regained. In October-November 1978, a slow summit deflation that totaled about 15 microradians may have resulted in an inflation documented by try-tilt measurements in the central east rift zone near the 1977 vents. Seismicity remained at moderately high levels in both the summit and east rift zone.

On Mauna Loa, geodimeter and dry-tilt measurements documented the continued slow inflation of the summit and northeast rift zone during the first half of the year. Borehole tiltmeter data showed a slight deflation of the latter area late in 1978. Seismicity remained generally low with no major earthquake swarms or single earthquakes larger than magnitude 4.5 .

Reoccupation of the Mauna Loa-Hualalai-Mauna Kea "Super Triangle" geodimeter lines showed horizontal changes lower than instrumental error among the three volcanoes. Geodimeter lines near the summit of Hualalai showed insignificant changes over two years. 


\section{SEISMIC INSTRUMENTATION}

The network. The Hawaiian Volcano Observatory has installed and maintains an extensive telemetering seismometer network on the island of Hawaii. In December 1978 the seismometer network consisted of 41 stations spread over an area with a diameter of 125 kilometers on the island of Hawaii (Figs. 1 and 2). Of these 41 stations, two are low-gain multicomponent stations (optical), six are two-component, three are three-component, and twenty-nine are vertical only. The coverage is most complete on and around the main center of seismic and volcanic activity, Kilauea Volcano. Other stations in the network are part of a larger net located on other volcanoes of the island of Hawaii. With the exception of HIL, all seismometer signals from the short period network are telemetered to the observatory for recording.

Figure 1 is a map of selected geographic and geologic features, and Figure 2 shows the seismic stations which were operated during the year. Table 1 lists all seismic stations operated by the U.S. Geological Survey in Hawaii during 1978. Listed are station name, three letter code, coordinates in degrees and minutes, elevation in meters, and other data described below.

Instrumentation and recording. Each telemetering station has a voltage controlled oscillator (VCO) for FM multiplex transmission to HVO via either hardwire or VHF radio. These telemetering stations are now all of Type 1, the NCER standard system used in USGS seismic networks (see Table 2 for details). After discrimination, the analog signals from thirty-six stations are recorded on two Develocorders using $16 \mathrm{~mm}$ microfilm. Smoked paper and helicorder drums additionally record a number of critical stations for convenience in rapid assessment of seismicity. FM signals from the telemetering network are also recorded directly on one inch magnetic tape. Selected larger events are copied onto condensed library tapes which are currently archived in Menlo Park.

Develocorder records are read on a film viewer with $20 x$ magnification. Arrivals are read to the nearest 0.05 second. The recorded arrival times, amplitudes (where readable), and other key data are routinely processed on an Eclipse computer which arrived at HVO in November 1978.

In addition to the standard stations, optical seismographs are maintained at Uwekahuna (HVO), Hilo, Maui, and on Oahu (Kipapa station operated by the Pacific Tsunami Warning Center). The less sensitive short period records are used primarily for amplitude measurements for magnitude calculations to supplement readings from $16 \mathrm{~mm}$ film. Optical seismographs listed in Table 1 are of four types. Types numbered three and four are electro-mechanical systems of high and low gain respectively. Hilo and Haleakala are each equipped with two lowgain Wood-Anderson torsion seismographs. Long period Press-Ewing seismographs record in three components in the Uwekahuna vault. The paper records as well as the $16 \mathrm{~mm}$ develocorder microfilm are archived at HVO.

Seismograph response and calibration. Displacement response curves for the four short-period seismograph types in use are given in Figure 3. Types three and four are electro-mechanical systems recorded on paper records. The Type 1 curve gives the displacement magnification of the standard NCER system from ground motion at the seismometer to the seismic trace as seen on a $20 \mathrm{x}$ Develocorder film viewer. The curves plot the unit response which should be multiplied by the factors CAL listed in Table 1 to get the response for an 
to the peak-to-peak amplitude measured in $\mathrm{mm}$ on the 20x Develocorder viewer of a 10 microvolt $5 \mathrm{~Hz}$ signal introduced to the preamp/VCO in place of the geophone. Calibration is normally done each time a station is visited, and major changes in attenuation or recalibrations are listed in Table 1 along with the dates they took place. Minor changes in CAL factors may not be listed if they are less than $20 \%$.

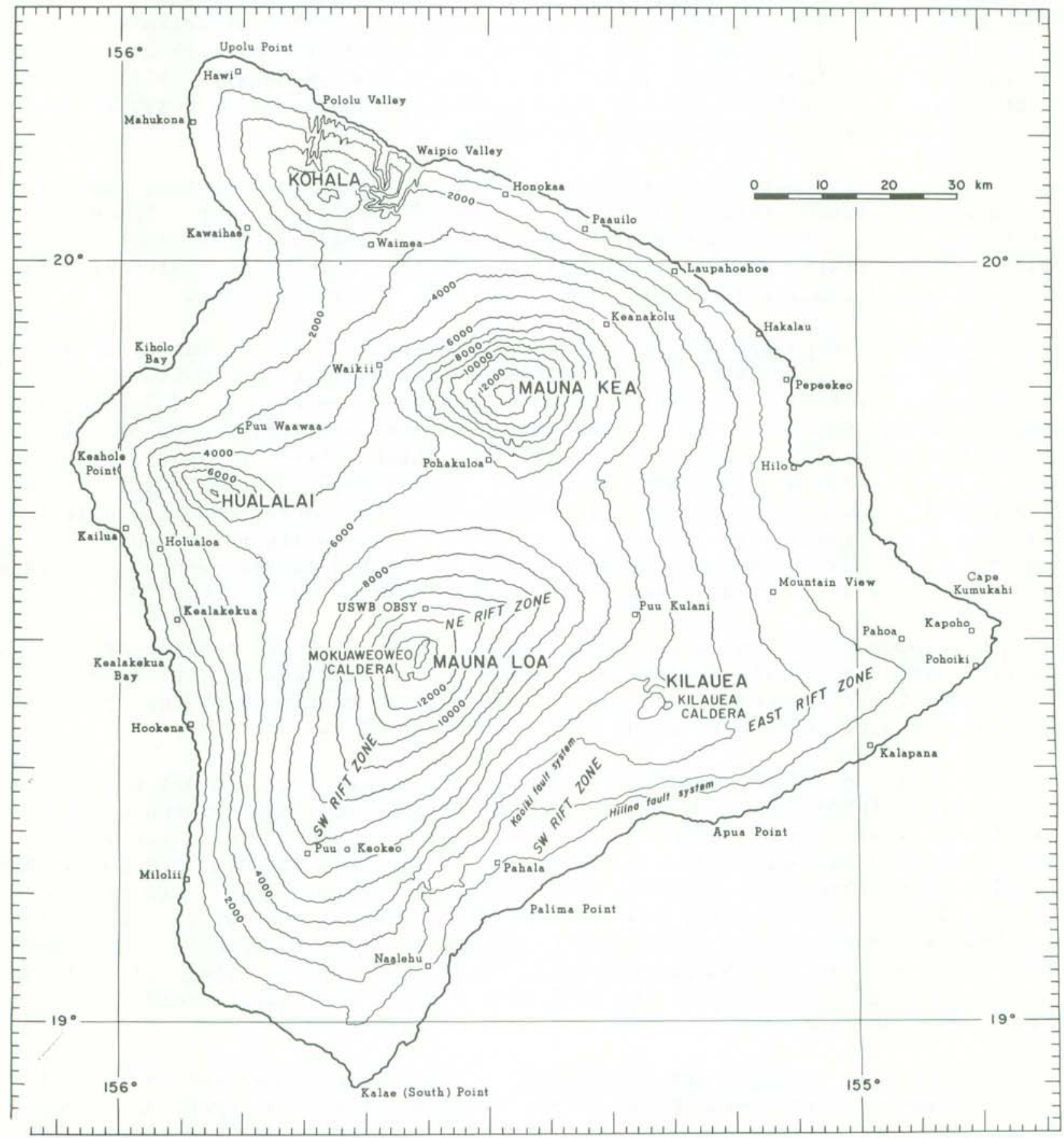

Figure 1. Map of the island of Hawaii showing principal settlements and selected geographic and geologic features. 


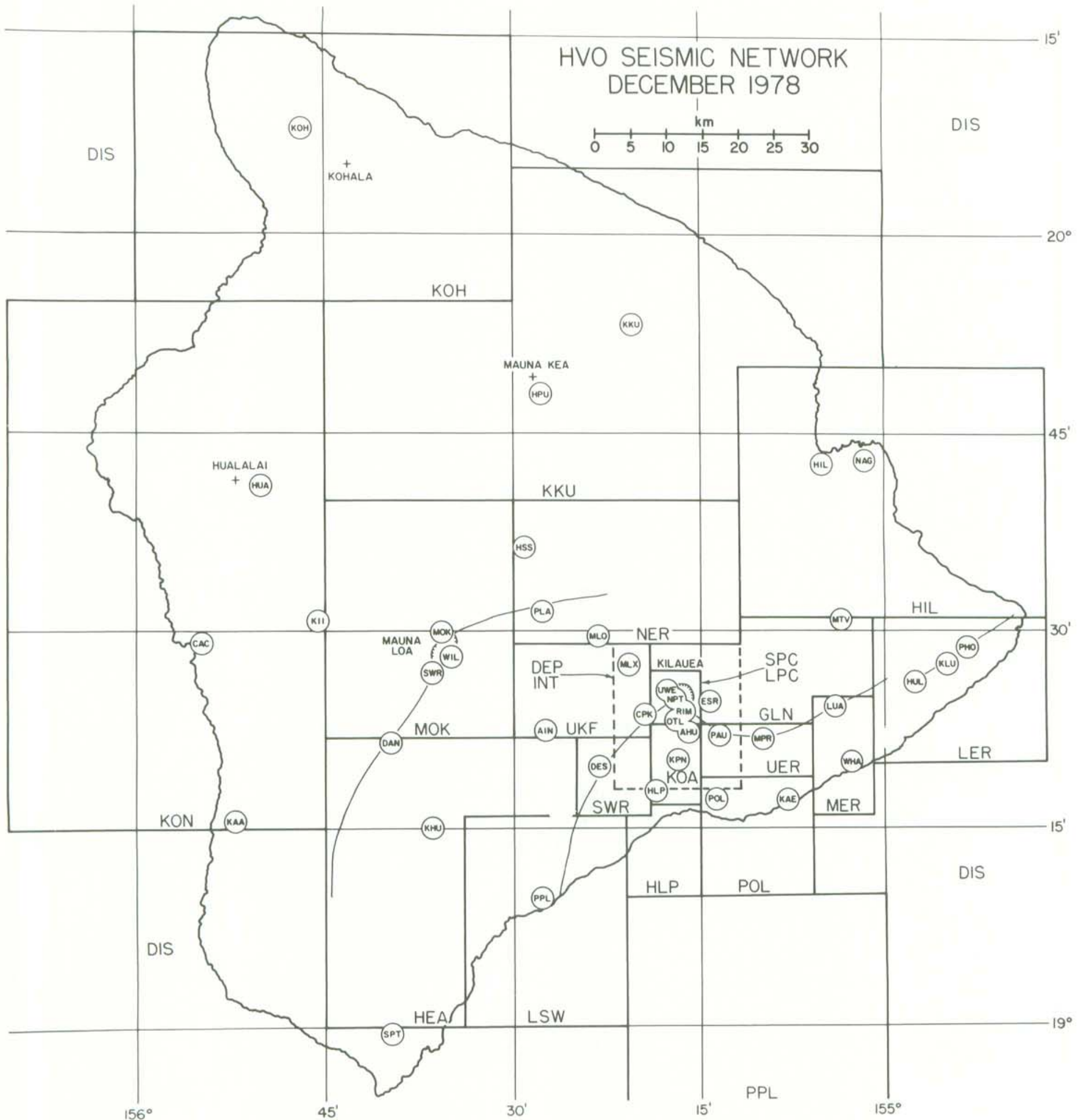

Figure 2. Map of the island of Hawaii showing seismic stations (three letter codes inside circles) and geographic regions (three letter codes inside heavy line boxes). 
Table 1. Seismometer stations in Hawaii operated by the U.S. Geological Survey, 1978.

Telemetered type 1 seismographs.

\begin{tabular}{|c|c|c|c|c|c|c|c|c|c|c|c|}
\hline Station Name ${ }^{1}$ & Code & LAT & {$[-\mathrm{N}$} & LON- & & Elev & $\begin{array}{c}\text { Delay } \\
1\end{array}$ & $\begin{array}{c}\text { Delay } \\
2\end{array}$ & $\begin{array}{l}\text { Old } \\
\mathrm{Cal}\end{array}$ & $\begin{array}{l}\text { Date of } \\
\text { Change }\end{array}$ & $\begin{array}{l}\text { New } \\
\mathrm{Cal}\end{array}$ \\
\hline AHUA & AHU & 19 & 22.40 & 155 & 15.90 & 1070 & -.10 & -.13 & $4 \cdot 3$ & & \\
\hline AINAPO (3) & AIN & 19 & 22.50 & 155 & 27.62 & 1524 & .13 & .17 & 8.5 & $1 / 10 / 78$ & 4.8 \\
\hline CAPTAIN COOK & CAC & 19 & 29.29 & 155 & 55.09 & 323 & .00 & -.16 & 11.0 & & \\
\hline CONE PEAK (2) & CPK & 19 & 23.70 & 155 & 19.70 & 1030 & -.26 & -.07 & 4.6 & $10 / 17 / 78$ & 6.0 \\
\hline DANDELION & DAN & 19 & 21.42 & 155 & 40.04 & 3003 & -.27 & .03 & 7.0 & $6 / 07 / 78$ & 6.0 \\
\hline DESERT & DES & 19 & 20.20 & 155 & 23.30 & 815 & -.29 & -.13 & 4.4 & & \\
\hline ESCAPE ROAD & ESR & 19 & 24.68 & 155 & 14.33 & 1177 & -.17 & -.19 & 1.2 & $11 / 28 / 78$ & 1.0 \\
\hline HALE POHAKU & HPU & 29 & 46.85 & 155 & 27.50 & 3396 & .31 & .17 & 3.3 & $10 / 13 / 78$ & 5.2 \\
\hline HE IHE IAHULU & HUL & 19 & 25.13 & 155 & 58.72 & 369 & -.17 & -.16 & 3.0 & $3 / 20 / 78$ & 2.2 \\
\hline HILINA PALI & HLP & 19 & 17.96 & 155 & 18.63 & 707 & .02 & .07 & 4.0 & & \\
\hline HUALALAI & HUA & 19 & 41.25 & 155 & 50.32 & 2189 & .67 & .38 & 3.8 & & \\
\hline HUMUULA (3) & HSS & 19 & 36.31 & 155 & 29.13 & 2445 & .20 & .35 & 8.4 & & \\
\hline KAAPUNA & KAA & 19 & 15.98 & 155 & 52.28 & 524 & -.12 & -.01 & 5.6 & & \\
\hline KAENA & $\mathrm{KAE}$ & 19 & 17.35 & 155 & 7.95 & 37 & -.01 & .06 & 1.3 & & \\
\hline KAHUKU & $\mathrm{KHU}$ & 19 & 14.90 & 155 & 37.10 & 1939 & .03 & -.03 & 3.2 & $8 / 23 / 78$ & 6.3 \\
\hline KALALUA & LUA & 19 & 24.55 & 155 & 04.25 & 622 & -.25 & -.30 & 4.6 & $7 / 05 / 78$ & 4.4 \\
\hline KALIU & KLU & 19 & 27.48 & 154 & 55.26 & 271 & -.17 & -.30 & -- & & \\
\hline KANEKII (3) & KII & 19 & 30.56 & 155 & 45.90 & 1841 & .15 & .37 & 5.5 & & \\
\hline KEANAKOLU & KKU & 19 & 53.39 & 155 & 20.58 & 1863 & .68 & .24 & 4.8 & $2 / 10 / 78$ & 2.0 \\
\hline KIPUKA NENE & KPN & 19 & 20.10 & 155 & 17.40 & 924 & -.11 & -.08 & 6.4 & & \\
\hline KOHALA & $\mathrm{KOH}$ & 20 & 7.69 & 155 & 46.77 & 1166 & -.03 & -.17 & 2.2 & & \\
\hline MAUNA LOA (2) & MLO & 19 & 29.80 & 155 & 23.30 & 2010 & .03 & .08 & 9.2 & & \\
\hline MAUNA LOA X (2) & MLX & 19 & 27.60 & 155 & 20.70 & 1474 & .06 & .15 & 2.1 & & \\
\hline MAKAOPUHI & MPR & 19 & 22.07 & 155 & 9.85 & 881 & -.17 & -.20 & 1.6 & $3 / 13 / 78$ & 1.3 \\
\hline MOKUAWEOWEO & MOK & 19 & 29.28 & 155 & 35.98 & 4104 & .15 & .16 & 8.0 & & \\
\hline MOUNTAIN VIEW & MTV & 19 & 30.25 & 155 & 3.75 & 409 & -.02 & .01 & 5.2 & & \\
\hline NATIONAL GUARD & NAG & 19 & 42.12 & 155 & 1.72 & 18 & .54 & .30 & $2.4 ?$ & $1 / 05 / 78$ & 11.0 \\
\hline NORTH PIT & NPT & 19 & 24.90 & 155 & 17.00 & 1115 & -.30 & -.18 & 5.2 & & \\
\hline OUTLET (2) & OTL & 19 & 23.40 & 155 & 16.80 & 1084 & -.19 & -.18 & 4.9 & & \\
\hline PAUAHI (2) & PAU & 19 & 22.62 & 155 & 13.10 & 994 & -.21 & -.24 & 6.2 & $10 / 15 / 78$ & 4.0 \\
\hline POLIOKEAWE PALI & POL & 19 & 17.02 & 155 & 13.47 & 169 & -.02 & .03 & 4.2 & $7 / 05 / 78$ & 2.5 \\
\hline PUU HOHUAULA & $\mathrm{PHO}$ & 19 & 28.90 & 154 & 53.4 & 215 & -.09 & -.24 & 4.2 & & \\
\hline PUU PILI & PPL & 19 & 9.50 & 155 & 27.87 & 35 & -.15 & -.15 & 2.4 & & \\
\hline PUU ULAULA & PLA & 19 & 32.00 & 155 & 27.67 & 2992 & -.03 & .13 & 7.4 & $6 / 07 / 78$ & 6.8 \\
\hline RIM (2) & RIM & 19 & 23.90 & 15 & 16.6 & 1128 & -.21 & -.13 & 7.3 & & \\
\hline SOUTH POINT & SPT & 18 & 58.91 & 155 & 39.92 & 244 & -.17 & -.22 & 3.6 & & \\
\hline SOUTHWEST RIFT & SWR & 19 & 27.26 & 155 & 36.30 & 4048 & .01 & .04 & 4.7 & $4 / 27 / 78$ & 9.0 \\
\hline WAHAULA & WHA & 19 & 19.90 & 155 & 2.92 & 29 & -.10 & -.04 & 2.3 & & \\
\hline WILKES CAMP & WIL & 19 & 28.15 & 155 & 35.02 & 4037 & .22 & .17 & 4.0 & $6 / 07 / 78$ & 3.7 \\
\hline
\end{tabular}

$1_{\text {Numbers }}$ in parentheses indicate the total number of seismometers operating if more than a vertical component. 
Table 1. (continued)

Optical Seismographs

\begin{tabular}{|c|c|c|c|c|c|c|}
\hline Station Name & Code & LAT $-\mathrm{N}$ & LON-W & Elev & Type & $\mathrm{Cal}$ \\
\hline HALEAKALA Z & HAL & 2046.00 & 15615.00 & 2090 & 3 & 0.71 \\
\hline HALEAKALA EW & HAE & 2046.00 & 15615.00 & 2090 & WA & 1.0 \\
\hline HALEAKALA NS & HAN & 2046.00 & 15615.00 & 2090 & WA & 1.0 \\
\hline HILO Z & HIL & 1943.20 & $155 \quad 5.30$ & 20 & 3 & 1.0 \\
\hline HILO EW & HIE & 1943.20 & 5.30 & 20 & WA & 1.0 \\
\hline HILO NS & HIN & 1943.20 & 5.30 & 20 & WA & 1.0 \\
\hline KIPAPA & KIP & 2125.40 & $158 \quad .90$ & 76 & 3 & 0.56 \\
\hline UWEKAHUNA EW & UWH & 1925.40 & 15517.60 & 1240 & 3 & 0.7 \\
\hline UWEKAHUNA Z & USZ & 1925.40 & $155 \quad 17.60$ & 1240 & 4 & 1.0 \\
\hline UWEKAHUNA EW & USE & 1925.40 & $155 \quad 17.60$ & 1240 & 4 & 1.0 \\
\hline UWEKAHUNA & PEZ & 1925.40 & $155 \quad 17.60$ & 1240 & $\mathrm{PE}$ & \\
\hline UWEKAHUNA & PEE & 1925.40 & $155 \quad 17.60$ & 1240 & PE & \\
\hline UWEKAHUNA & PEN & 1925.40 & $155 \quad 17.60$ & 1240 & $\mathrm{PE}$ & \\
\hline
\end{tabular}

Table 2. -- Seismic Instrumentation Types

Type 1. Consists of:

a) Geophone - Electrotech EV-17 or Mark Products L4C $1.0 \mathrm{sec}$. period vertical component seismometer or horizontal component adjusted for an output of 0.5 volts $/ \mathrm{cm} / \mathrm{sec}$. and 0.8 critically damped.

b) Preamp/VCo - Develco Model 6202 voltage controlled oscillator or a USGS/NCER Model J202 or J302. $3 \mathrm{db}$ points for bandpass filter at $0.1 \mathrm{~Hz}$ and $30 \mathrm{~Hz}$. Signals are transmitted on audio FM carrier over cable or FM radio link to HVO.

Type 3. Consists of:

Electrotech EV-17 or observatory-built $0.8 \mathrm{sec}$. period moving coil seismometer with HVO-built solid state seismic preamplifier, galvanometer driver, and $2 \mathrm{~Hz}$ galvanometer. Peak magnification approximately 40,000 at $4 \mathrm{~Hz}$.

Type 4. Consists of:

Sprengnether short period vertical and horizontal seismometers (E-W) with $1.5 \mathrm{sec}$. galvanometers, coupling factor $=0.25,2 \mathrm{X}$ critically damped. Peak magnification approximately $1500 \mathrm{X}$ at $2 \mathrm{~Hz}$.

Type 2 instruments have been discontinued. 


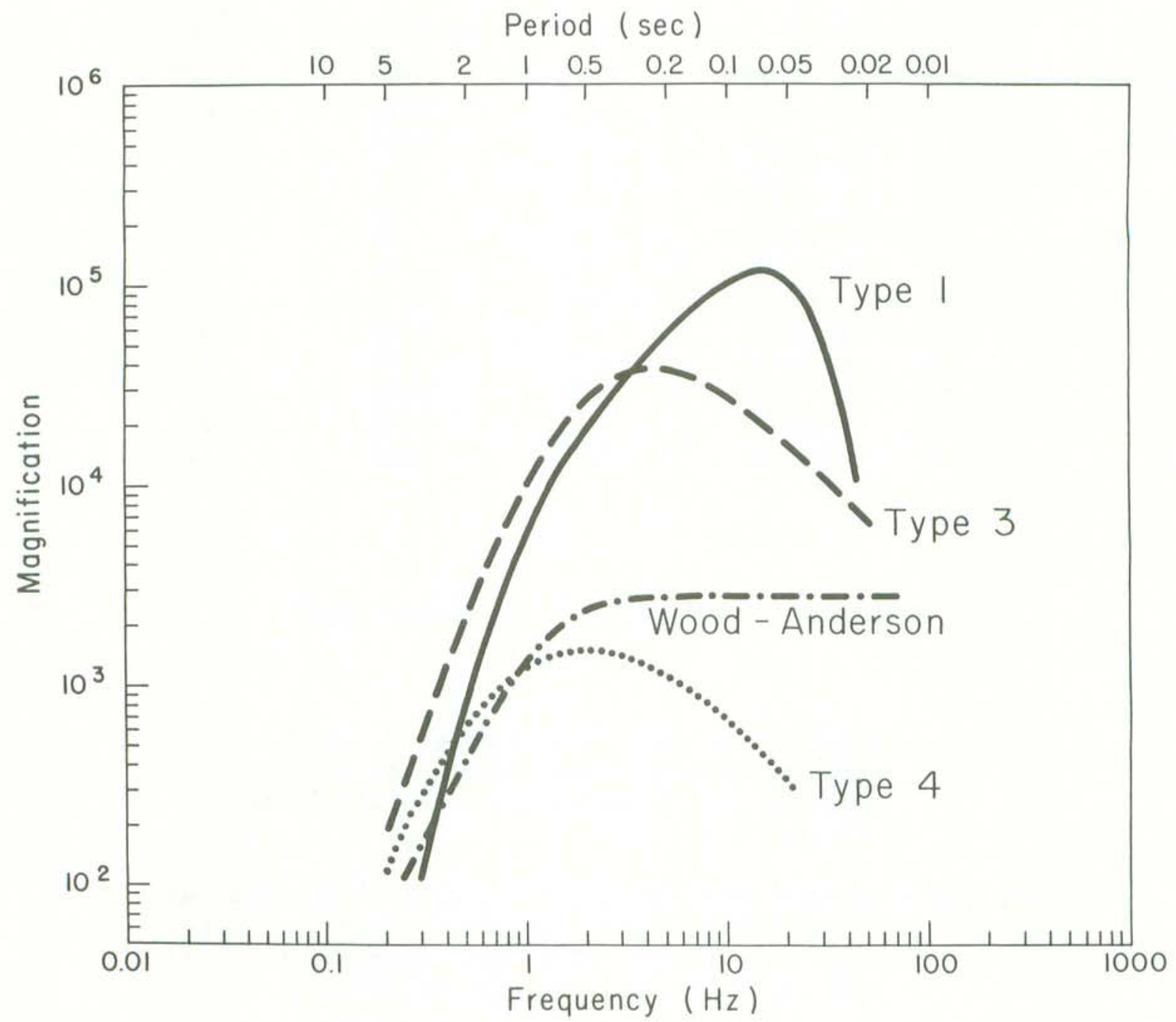

Figure 3. System response curves for the Wood-Anderson torsion seismograph and for the 3 different types of seismometers in use by the Hawaiian Volcano Observatory. Types 3 and 4 are electro-mechanical seismographs recorded optically on photographic paper. Type 1 is the standard NCER seismometer recorded on Develocorder film and magnetic tape. The curve for Type 1 includes response of the geophone, all electronics including telemetry, Develocorder galvanometer, and projection of film by a $20 x$ viewer. The curves plot the unit response which should be multiplied by the factors CAi listed in Table 1 to get the response for an individual station. 


\section{SEISMIC DATA PROCESSING}

Earthquakes are located by the combined efforts of HVO and NCER in Menlo Park. Develocorder films are read at HVO, and $P$ and $S$ arrival times, event amplitude and duration, clock correction, etc. are input and located on the Eclipse computer at HVO. Computer locations are made using the program HYPOINVERSE (Klein, 1978), and problem events are reread and rerun. Magnetic tape copies of all arrival time and output summary data are kept in Menlo Park and HVO.

The crustal model used is specified by velocities at 4 depth points. Velocity at any depth is given by linear interpolation between points and uses a homogeneous half-space below.

\begin{tabular}{cc}
$\begin{array}{c}\text { VELOCITY } \\
(\mathrm{km} / \mathrm{sec})\end{array}$ & DEPTH $(\mathrm{km})$ \\
\cline { 2 - 2 } 1.9 & 0.0 \\
6.5 & 4.6 \\
6.9 & 15.0 \\
8.3 & 16.5
\end{tabular}

Two empirical sets of station delays or corrections were used in the locations, and are given in Table 1. Delay model 1 is used for events on Kilauea and its south flank, and delay model 2 applies to the rest of the island and offshore earthquakes. The delay models are in fact separated by a circle of radius $34 \mathrm{~km}$ centered at $19^{\circ} 22^{\prime} \mathrm{N}$ and $155^{\circ} 10^{\prime} \mathrm{W}$.

Magnitudes for most events were computed using both recorded amplitudes on low gain or Wood-Anderson stations and signal or coda duration on short-period vertical stations. Amplitudes read from other than Wood-Anderson instruments are corrected to an equivalent Wood-Anderson amplitude using the curves of Figure 3 and CAL factors of Table 1. Amplitude magnitudes larger than 2.5 are generally based on the Wood-Anderson instruments in Hilo or Type 4 seismographs at Uwekahuna. Smaller events may occasionally include amplitude readings from stations AHU, KAA, OTL, PPL, KHU, PHO, or WIL.

Duration magnitudes are determined from the length of signal in seconds read from the Develocorder viewer. This time, also called the "F-P time" is measured from the first $P$ arrival to the point where the earthquake signal has decayed nearly to the noise level. A plot of log (F-P time) versus local (amplitude) magnitude appears in Figure 4. The bilinear relation shown in the figure is an appropriate fit to the data sample and is used to compute all duration magnitudes. Duration times are only read from Type 1 seismographs. Because duration magnitudes are relatively insensitive to station response and can be determined using the high-gain short-period stations, it is felt that duration magnitudes are more accurate and complete at the lower magnitudes (below 2). 


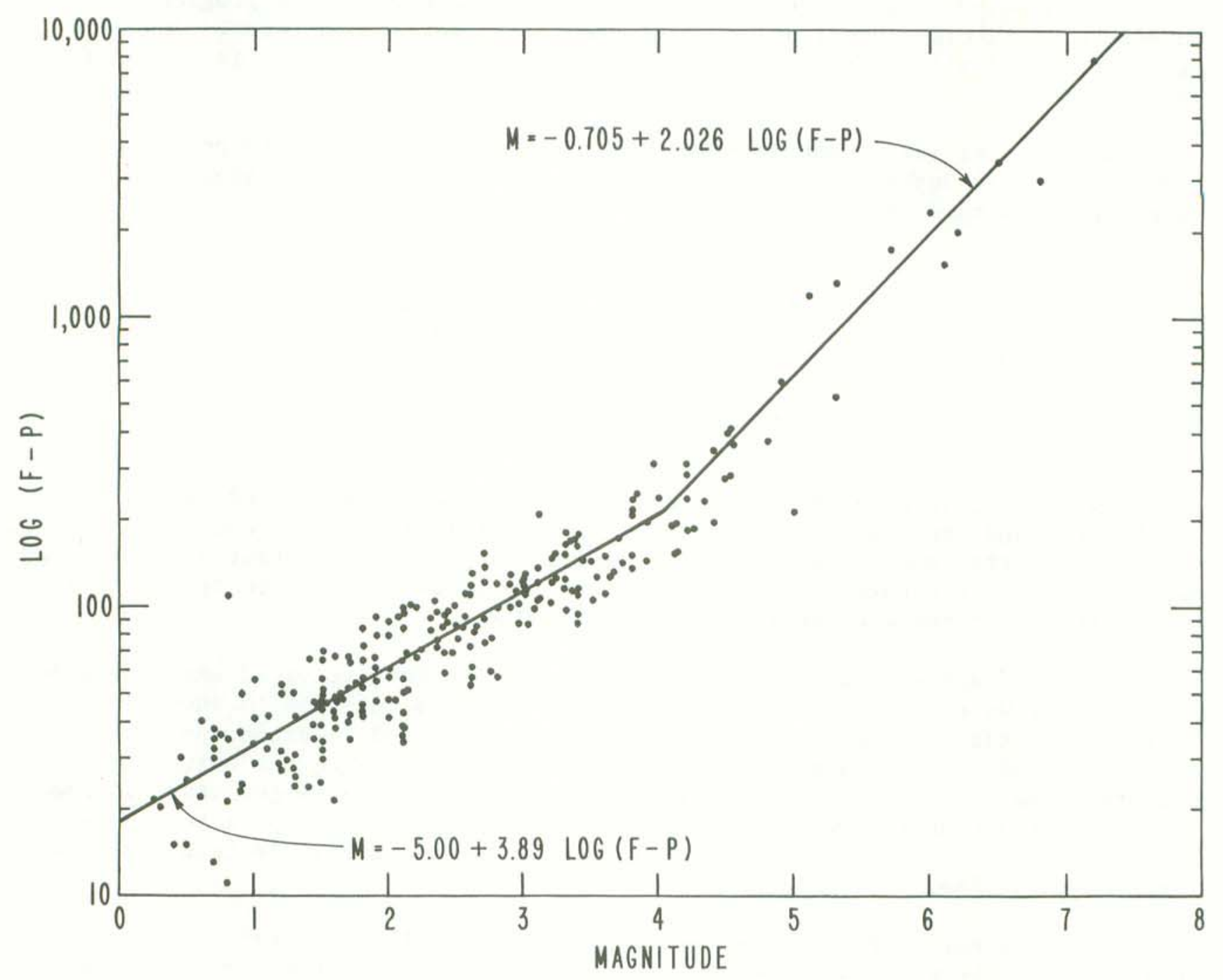

Figure 4. Relationship between signal duration (F-P time) and local magnitude for a large number of earthquakes which occurred during 1975 and 1976. Local magnitude is determined from amplitudes read on Wood-Anderson and other calibrated seismographs. The dual linear relationship between magnitude and $\log (\mathrm{F}-\mathrm{P})$ appears to hold over a magnitude range of 7 units. The equations used in magnitude determination are:

$$
\begin{aligned}
& \text { duration }<210 \mathrm{sec} \quad M=-5.2+3.89 \log (\mathrm{F}-\mathrm{P})+.013 \mathrm{z}+.0037 \mathrm{D} \\
& \text { duration }>210 \mathrm{sec} \quad M=-.905+2.026 \log (\mathrm{F}-\mathrm{P})+.013 \mathrm{z}+.0037 \mathrm{D} \\
& \text { where } z \text { and } D \text { are the depth and epicentral distance in km. }
\end{aligned}
$$


The emphasis in both station coverage and detailed data analysis is on the highly active south flank of the island of Hawaii. Hundreds of earthquakes too small to locate are counted daily, and the set of located earthquakes in the Kilauea region is nearly complete above magnitude about 2.0. Many smaller events are located also. Substantial effort is made to locate earthquakes elsewhere on the island and within about $150 \mathrm{~km}$ of the island. Such coverage cannot be as complete as on Kilauea, but nearly all events above magnitude 3.0 to 3.5 are located.

Data presented in the seismic summary is in four parts. Table 3 gives duration of harmonic tremor and numbers of earthquakes (most too small to locate) from several source regions around Kilauea. The source region is determined visually from signal character and pattern of arrival times at key stations. Maps showing computer located epicenters of all depths are given in Figures 5, 6, 7, 8 and 9. The epicenter maps are on different scales, and show both all located earthquakes and large events only.

The list of computer locations constitutes the bulk of this summary, and is given in Table 5. Each earthquake in the list is assigned a three-letter code based on its location and in some cases on its depth. Figure 2 is a map of the regions used to assign the location codes. The latitude and longitude limits of rectangular regions are listed in Table 4. When the listed coordinates imply an overlap, precedence is given according to Figure 2. Table 6 relists the events in Table 5 for which either duration or amplitude magnitude is 3.0 or larger. It is felt that this list is a more objective measure of large earthquakes than a list of felt earthquakes. 
Table 3. Number of earthquakes and minutes of tremor recorded on seismographs around Kilauea.

Earthquake categories are:

1) Kilauea summit, short period caldera (SPC): shallow earthquakes beneath the caldera.

2) Kilauea summit, long period caldera (LPC): earthquakes characterized by low frequency signitures, often originating $5-10 \mathrm{~km}$ below the caldera.

3) Kilauea summit $30 \mathrm{~km}$ (DEP): from about $30 \mathrm{~km}$ depth.

4) Kaoiki and southwest rift (UKF/SWR): southwest rift of Kilauea and adjacent Kaoiki fault system.

5) Upper east rift zone of Kilauea including the Koae faults (UER).

6) Lower east rift zone of Kilauea (LER).

7) Offshore PPL: earthquakes from offshore areas south of the Puu Pili station, including Loihi seamount (PPL).

8) Mauna Loa long period (MLP): low frequency events near Mauna Loa summit.

9) Mauna Loa short period (MOK): shallow earthquakes in the Mauna Loa caldera region.

Tremor is separated into four categories: shallow, intermediate and deep Kilauea, and Mauna Loa. Depth is inferred on the basis of relative amplitudes on seismographs in the Kilauea summit region.

Dates of Station Outage or Conditions Affecting Seismic Count

Date

$\underline{(\mathrm{Y}-\mathrm{M}-\mathrm{D})}$

$78-5-9$

$78-5-20$

$78-7-18 / 21$

$78-11-6 / 8$

$78-11-13$

$78-12-20$

$78-12-25 / 26$
Nature of Disturbance

High winds, stations out--HUL, PHO, PAU, PLA

Electrical storm, stations out--WHA, ESR, AHU

High winds

High winds, electrical storm, many stations out Film recorder 4-hour outage

High winds

Film recorder jammed-15 hours
Count Category

Affected

SPC, UKF/SWR,

UER

UER

SPC, UER, UKF / SWR

All counts at

Kilauea

Al1 counts

SPC

Al1 counts 
TABLE 3 .

KILAUEA FLANK

MdUNA 100 TREMOR (MINUTES)

\begin{tabular}{|c|c|c|c|c|c|c|c|c|c|c|c|c|c|c|c|c|c|}
\hline \multicolumn{2}{|l|}{ I } & \multicolumn{2}{|c|}{ ISHORT } & LONG & \multirow{2}{*}{30} & \multicolumn{2}{|c|}{ I $K A O$. } & \multirow{2}{*}{$\begin{array}{l}\text { UP. } \\
\text { FAST }\end{array}$} & LOW. & OFF - & \multicolumn{2}{|c|}{ I } & & $T$ & KILAUEA & \multicolumn{2}{|c|}{ MAUNII } \\
\hline DAT & TE & $I$ & PER. & PFP. & & I & $\& S W$ & & EAST & SHORE & $E I L$. & 17 & SHก DT & Y & INiT. & LOA & I \\
\hline 197 & 78 & 1 & CALI & DFRA & $K M$ & $\mathrm{I}$ & $R$ IF T & R IF T & PIFT & $P P L$ & IPF & r. & PFP. & ! & SHAL. & DEEP & \\
\hline \multicolumn{18}{|c|}{ 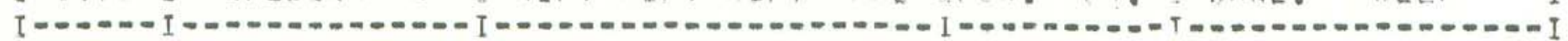 } \\
\hline \multicolumn{18}{|c|}{ IJAN 1 I $124 \quad 4 \quad$ I $45 \quad 152$ L $15 \quad$ I } \\
\hline & 2 & $\mathrm{~T}$ & 242 & 15 & 1 & $\mathrm{~T}$ & 41 & 225 & 5 & & I & & & T & 5 & 14 & \\
\hline & 3 & I & 259 & 20 & 1 & 1 & 28 & 220 & 20 & & I & & 1 & 1 & 7 & 2 & \\
\hline & 4 & I & 251 & 24 & 2 & I & 47 & 253 & 14 & & I & 1 & 3 & 1 & 12 & 25 & \\
\hline & 5 & I & 2.21 & 3 & 1. & I & 45 & 151 & 23 & & 1 & ? & 2 & $T$ & 11 & 5 & \\
\hline & $\infty-$ & $\mathrm{I}$ & & & & -1 . & $=-$ & $-\infty$ & $\ldots$ & & $I$ & & $--0 .-\infty$ & & $-\infty-=--=-\infty$ & $-----m$ & \\
\hline & 6 & I & 234 & 29 & & I & 42 & 190 & 13 & & I & & 1 & T & 5 & 3 & \\
\hline & 7 & I & ટટટ & 9 & & I & 42 & 193 & 5 & & I & h & & 1 & 3 & & \\
\hline & $n$ & I & 260 & 18 & & I & 46 & 2दी & 12 & & I & 3 & & $r$ & 34 & & \\
\hline & 9 & I & 188 & 3 & 1 & I & $4 h$ & 203 & 12 & & I & 4 & 2 & ! & ¿1 & & \\
\hline & 10 & I & 188 & 11 & & I & 51 & 276 & 23 & & I & & & ? & & & \\
\hline$-\infty$ & --- & $\mathrm{I}$ & ---- & $=-$ & $-\infty$ & & $=-\infty$ & $=--\infty$ & & & $-I=$. & & $-0-0$ & & $-\infty$ & $=--$. & \\
\hline & 11 & I & 132 & ? & ? & $\mathrm{I}$ & 42 & 147 & 9 & & I & & & 1 & 10 & & \\
\hline & $1 ?$ & I & 251 & 9 & 4 & I & 54 & 241 & 18 & & I & & & r & & & \\
\hline & 13 & I & 180 & 16 & 1 & I & 18 & 125 & 34 & & I & ?? & 1 & T & & 5 & \\
\hline & 14 & I & 163 & 6 & 1 & I & 23 & $9 n$ & 41 & & I & 15 & $?$ & $T$ & 6 & & \\
\hline & 15 & $\tau$ & 276 & 6 & 1 & I & 52 & 218 & 17 & & I & & & $r$ & 7 & & \\
\hline & $=--$ & I - & $=-\infty$ & & $=$. & $-I$ & $=-\infty$ & 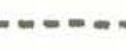 & & & -1 & $=-$ & $-\infty-$ & & & - & \\
\hline & 16 & I & 259 & 3 & 1 & $\tau$ & 38 & 191 & 8 & & I & 1 & 1 & I & 3 & & \\
\hline & 17 & I & 78 & ? & & I & 28 & 115 & 5 & & I & & & ? & & & \\
\hline & 18 & I & 189 & 3 & 1 & I & 34 & 149 & 5 & & I & 2 & 15 & 1 & ? & & \\
\hline & 19 & I & $19 ?$ & 1 & ? & I & 33 & 140 & 10 & & I & 1 & $2 ! 1$ & $T$ & 26 & & \\
\hline & 20 & I & 295 & 7 & & I & 53 & 164 & 9 & & I & & ง & 1 & 5 & 4 & \\
\hline & $\ldots-\infty$ & 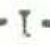 & $=-\infty$ & & & & $=-$ & $=-\infty$ & & & $-I^{2}$ & & $\ldots$ & & & & \\
\hline & 21 & I & 301 & 14 & & T & 60 & 192 & \& & & $I$ & & & T & 2 & 2 & \\
\hline & 22 & I & 304 & 16 & 3 & I & 53 & 210 & 17 & & I & & 1 & T & & & \\
\hline & 23 & I & 180 & 5 & 3 & I & 37 & 95 & 5 & & I & 2 & \& & $T$ & 8 & & \\
\hline & 24 & I & 300 & $1 ?$ & ट & T & 51 & 156 & 19 & & I & 2 & 1 & I & 5 & & \\
\hline & 25 & $T$ & 235 & 4 & & I & 36 & 128 & 22 & & $\mathrm{I}$ & 1 & & T & 2 & & \\
\hline & $=--$ & $\mathrm{I}$ & 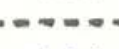 & & & $-I$ & $=-\infty$ & $--\infty-\infty$ & & & $-I$ & & $-\infty$ & T - & & & \\
\hline & 26 & I & 198 & 2 & 1 & 1 & 38 & 159 & 21 & & I & & 3 & $T$ & & & \\
\hline & २.7 & I & 219 & 4 & & 1 & 45 & 178 & 26 & & I & & $>$ & $T$ & & & \\
\hline & 28 & 1 & 225 & 8 & & I & 57 & 166 & 15 & & I & & 5 & $T$ & 7 & & \\
\hline & 29 & I & 269 & 29 & 3 & I & 46 & 184 & 11 & & I & 3 & 2 & $T$ & & & \\
\hline & 30 & $\mathrm{~T}$ & 300 & 6 & 2 & I & $\Delta 9$ & 237 & 17 & & I & 1 & $?$ & I & & & \\
\hline & $-\infty-$ & I & & & & & $\infty$ & $=-\infty$ & & & & & $=-\infty$ & & 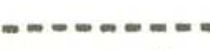 & & \\
\hline T 3 & 31 & $\tau$ & 288 & 14 & 1 & I & 54 & 194 & 15 & & 1 & 4 & ? & $T$ & 5 & & \\
\hline [FEP & 1. & I & 310 & 5 & & I & 37 & 170 & 12 & & I & & 1 & & & & \\
\hline$\tau$ & 2 & [ & 351 & 15 & 5 & $T$ & 37 & $15 ?$ & 12 & & I & & 3 & $T$ & 2 & & \\
\hline & 3 & I & 265 & 5 & & I & 43 & 169 & 18 & & I & & ? & 1 & 30 & & \\
\hline & 4 & I & 145 & 9 & & I & 17 & 93 & उกิ & & I & $?$ & 3 & 1 & in & & \\
\hline$\tau=-\infty$ & -- & 1 & $--=-$. & & & & $--\infty$ & $=-\infty$ & $--\infty$ & & $-I-$ & &..$---n$ & & $10-0-0=0$ & $--\infty--$ & \\
\hline & 5 & I & 276 & 2 & 4 & I & 37 & 183 & 19 & & $\mathrm{I}$ & & 1 & $T$ & 6 & & \\
\hline & 6 & I & 250 & 3 & & I & 59 & 170 & 17 & & I & $?$ & ? & $T$ & & & \\
\hline & 7 & I & 265 & 5 & 3 & I & G2 & 181 & 15 & & I & & & $T$ & & 4 & \\
\hline & 8 & I & 96 & 7 & & I & 34 & 104 & 8 & & I & 1 & $?$ & T & & & \\
\hline 1 & 9 & $T$ & 124 & 5 & 4 & I & 24 & 118 & 10 & & I & & 3 & T & 14 & & \\
\hline & $-m-$ & - I - & $-\infty$ & & & & $=-\infty-\infty$ & $-----\infty$ & $---\infty$ & & & & - - - - = & & ten & $--n-0$ & \\
\hline & 10 & $T$ & 240 & 12 & 1 & $\mathrm{I}$ & 28 & 150 & 19 & & I & & 1 & I & 15 & 2 & \\
\hline & 11 & I & 222 & Q & 5 & I & 26 & 156 & 7 & & 1 & & 3 & I & 17 & & \\
\hline 1 & 12 & I & 257 & 16 & & I & 28 & 172 & 9 & & I & 3 & 1 & + & & 93 & \\
\hline 1 & 13 & I & 214 & 5 & & $\tau$ & 41 & 155 & 3 & & $\tau$ & 1 & 1 & 1 & 38 & & \\
\hline & 14 & I & 270 & 21 & 5 & I & 35 & 184 & 12 & & $\mathcal{L}$ & 5 & 1 & $T$ & 3 & & \\
\hline
\end{tabular}


KILAUEA SUMMIT KILAUEA FLANK

\begin{tabular}{|c|c|c|c|c|c|c|c|c|c|c|c|c|c|c|c|c|}
\hline I & Is & BHORT & I.ONG & & 1 & KAO. & UP• & LOW. & $O F F=$ & I & & & I & KIL $\triangle U E A$ & & $J N \Delta I$ \\
\hline I DATE & I & PER. & PER. & 30 & I & $\& S$ S & EAST & EAST & SHORE & IL & $N G$ & SHOLT & $T$ & INT & & A I \\
\hline 1978 & I & CALD & ERA & $\mathrm{KM}$ & I & RIF T & R IF T & RIF T & PPL & IPr & : & PER. & I & SHAL. D & DEFP & \\
\hline & & & & $=0$ & & $1--m-2$ & $0-\infty-\infty$ & $=-0-\infty$ & & $1=$ & $=-\infty$ & $--\infty-\infty$ & & $--------\infty-\infty$ & $-\infty-\infty$ & $=-$ \\
\hline IFEB 15 & I & 264 & 15 & 3 & I & 46 & 197 & 13 & & I & 8 & & T & 7 & 9 & \\
\hline I 16 & I & 214 & 13 & & I & 36 & 943 & 9 & & I & 3 & 3 & $\top$ & 18 & & \\
\hline 17 & I & 268 & 2 & & I & 41 & 164 & 27 & & I & 3 & $?$ & $T$ & & & \\
\hline 18 & I & 309 & 26 & & I & 33 & 202 & 19 & & I & 2? & ? & $T$ & & & \\
\hline 19 & I & 269 & 5 & 2 & I & 63 & 210 & 15 & & t & 21 & 5 & I & & & 7 \\
\hline$\infty-\infty$ & - I . & $-\infty=-\infty$ & & $\infty$ & - I. & $-\infty=-\infty$ & 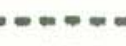 & 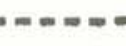 & & $1=$. & $\cdots$ & $-\infty+\infty=$ & & $---\infty$. & & \\
\hline 20 & I & 335 & 1 & & I & 44 & 155 & 15 & & I & $?$ & 15 & 1 & 13 & & \\
\hline 21 & I & 342 & 3 & & I & 44 & 245 & 11 & & I & 26 & 12 & Y & 5 & & \\
\hline 22 & I & 242 & & & I & 36 & 152 & 15 & & I & 1 & 15 & 1 & & & \\
\hline 23 & I & 254 & 2 & & I & 39 & 216 & h & & I & 14 & 1 & $\mathrm{~T}$ & & 32 & \\
\hline 24 & I & 279 & 4 & & I & 48 & 170 & 19 & & r & 1 & 5 & T & 7 & & \\
\hline$-\infty-\infty-\infty$ & $-I$. & $0-\infty$ & $-\infty$ & $-=-$ & $I$ * & $=0-\infty$ & $-\infty-\infty$ & $\infty$ & & $I \ldots$ & $=-\infty$ & $0=-\infty$. & T - & $-\infty-\infty$ & & $-\infty$ \\
\hline 25 & I & 157 & 3 & 1 & I & 29 & 99 & 30 & & I & 8 & 4 & $T$ & 3 & & 5 \\
\hline 26 & I & 305 & 14 & & I & 62 & 229 & 20 & & I & 9 & 3 & I & & & \\
\hline 27 & I & 297 & 5 & & I & 40 & 225 & 19 & & $\mathrm{~T}$ & & & 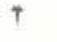 & & & \\
\hline 28 & I & 251 & 1 & & I & 34 & 263 & 31 & & I & & & T & 3 & & \\
\hline MAR 1 & I & 201 & 4 & & I & 35 & 246 & 6 & & I & & & I & 4 & & \\
\hline$\infty-\infty-\infty$ & $-I$ & $=-\infty-0$ & & $-\infty$ & I & $--\infty-\infty$ & $-----m$ & $=--\infty$ & & $I=$ & $-\infty$ & $-\infty-\infty$ & & $-m------\infty-\infty$ &.- & $-\infty$ \\
\hline 2 & I & 225 & 3 & & I & 54 & 183 & 9 & & I & 1 & & $\mathrm{~T}$ & 15 & & \\
\hline 3 & I & 202 & 4 & & I & 31 & 150 & 8 & & I & 3 & ? & $T$ & 21 & & \\
\hline 4 & I & 270 & 5 & & I & 49 & 175 & 23 & & I & & 3 & $T$ & & & \\
\hline 5 & I & 313 & 10 & 6 & I & 42 & 209 & 14 & & I & 2 & 7 & $\mathrm{~T}$ & 18 & & 7 \\
\hline 6 & I & 304 & 7 & 4 & I & 52 & 174 & 6 & & $\mathrm{I}$ & & 1 & T & 8 & & \\
\hline$--=-$ & - I. & $--\infty-\infty-2$ & $-\infty-\infty$ & $-\infty$ & $I$ & $\ldots-\infty$ & $-\infty-\infty$ & $=-\infty$ & & $1=0$ & $-\infty$ & $-\infty=-\infty$ & $1=$ & 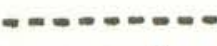 & $-\infty$ & $-\infty$ \\
\hline 7 & I & 244 & 14 & & I & 34 & 163 & 9 & & $I$ & & 1 & $T$ & 2. & 6 & \\
\hline 8 & I & 183 & 1 & 2 & I & 37 & 124 & 12 & & I & 1 & 2 & 1 & & & \\
\hline 9 & I & 259 & & & I & 34 & 149 & 7 & & I & & 1 & 1 & h & & \\
\hline 10 & I & 219 & 2 & & I & 17 & 141 & 7 & & 1 & & $?$ & I & 9 & & \\
\hline 11 & I & 249 & 9 & 1 & $\mathrm{~J}$ & 27 & 143 & 6 & & 1 & & & $T$ & & & \\
\hline 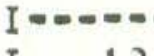 & I. & $-=-\infty$ & & $-\infty$ & $\mathrm{I}-$ & $-\infty$ & $=-\infty$ & $-\infty$ & & & $-\infty-$ & $-0-0 .-$ & $1-$ & $--------\infty$ & $---\infty$ & \\
\hline 12 & I & 214 & 9 & & I & 31 & 126 & 3 & & I & & 1 & $T$ & & & \\
\hline 13 & I & 324 & 11 & 3 & I & 26 & 184 & 3 & & I & & & I & & ? & \\
\hline 14 & I & 263 & 1 & 1 & I & 39 & 173 & 2 & & I & & $?$ & T & & 6 & \\
\hline 15 & I & 131 & 2 & & I & 38 & 129 & 3 & & I & 2 & 1 & $T$ & & & \\
\hline $16^{\circ}$ & I & 98 & 4 & & I & 13 & 134 & 5 & & I & & 1 & I & 2 & & \\
\hline$-0=-5$ & I - & $-0-1-2$ & & $-\infty$ & - I - & $--=-$ & $-2=-$ & $--\infty-\infty$ & & & $0=-\infty$ & $---\infty$ & & $---\infty-0=-0$ & $-\infty-$ & \\
\hline 17 & I & 182 & 2 & & I & 2.6 & 139 & 14 & & I & & 1 & 1 & 23 & & \\
\hline 18 & I & 70 & & 1 & I & $1 ?$ & 79 & 5 & & 1 & & & $T$ & 8 & & \\
\hline 19 & I & 275 & 14 & 4 & I & 32 & 159 & 5 & & I & & 1 & 1 & & 14 & \\
\hline 20 & I & 317 & 10 & 3 & I & 40 & 143 & 11 & & I & & & $T$ & & & \\
\hline 21 & I & 345 & 28 & 1 & I & 39 & 186 & 7 & & I & & 2 & i & & 15 & \\
\hline----0. & I & $-\infty-\infty$ & $--\infty$ & $-\infty$ & I - & 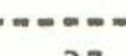 & $---m$ & & & I - & $=-$ & ----- & I - & - & $-\infty$ & \\
\hline 22 & I & 270 & 5 & 1 & I & 27 & 115 & H & & I & 1 & 2 & $i$ & 7 & & \\
\hline 23 & I & 249 & 4 & & I & 30 & 118 & 6 & & I & & ? & $r$ & & & \\
\hline 24 & I & 226 & 4 & & I & 30 & 135 & 22 & & $T$ & 1 & ? & $\tau$ & 13 & & \\
\hline 25 & I & 218 & 2 & & I & 24 & 134 & 19 & & 1 & & & $\uparrow$ & & & \\
\hline 26 & I & 350 & 9 & 4 & I & 46 & 201 & 10 & & 1 & & & 1 & & 29 & \\
\hline---5 & & 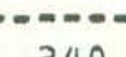 & $=-\infty$ & & $\mathrm{I} \cdot$ & $---\infty$ & $-x$ & $-\infty$ & $---n$ & $1=$ & - & $-\infty-\infty$ & & 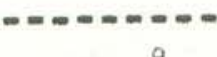 & 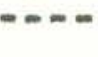 & \\
\hline 27 & I & 240 & 13 & & I & 34 & 122 & 21 & & I & & 1 & $T$ & 8 & & \\
\hline 28 & I & 263 & 8 & & I & 25 & 182 & 10 & & I & & ? & $T$ & & h? & \\
\hline 29 & I & 144 & 2 & & I & 19 & 88 & 8 & & I & & 1 & 1 & & & \\
\hline 30 & I & 186 & 4 & & I & 20 & 112 & 14 & & I & & & T & & & \\
\hline 31 & I & 159 & 16 & & I & 19 & 127 & 10 & & I & & 1 & I & 3 & & \\
\hline
\end{tabular}




\begin{tabular}{|c|c|c|c|c|c|c|c|c|c|c|c|c|c|c|c|c|c|c|}
\hline \multicolumn{2}{|l|}{ I } & \multicolumn{2}{|c|}{ ISHORT } & \multicolumn{2}{|l|}{ LONG } & $\mathrm{I}$ & KAO. & \multirow{2}{*}{$\begin{array}{l}\text { UP. } \\
\text { EAST }\end{array}$} & LOW. & \multirow{2}{*}{$\begin{array}{l}\text { DFF - } \\
\text { SHURF }\end{array}$} & \multicolumn{2}{|l|}{$I$} & \multicolumn{2}{|c|}{$I$} & \multicolumn{2}{|c|}{ KILAUEA } & \multicolumn{2}{|c|}{ MAUNAI } \\
\hline I & DATE & I & PER. & $P F R$. & 30 & I & $\& \mathrm{SW}$ & & EAST & & ILO & I & SHORT & $T$ & INT. & & LOA & I \\
\hline I & 1978 & I & CALD & ERA & $K M$ & I & RIF T & RIF T & RIFT & $P P L$ & IPF & . & PFR. & $I$ & SHAL. $\quad$ D & EEP & & I \\
\hline & $=--\infty=$ & $I$. &.----- & $=-\infty-\infty$ & $=-\infty$ & $I=$ & $-\infty=-\infty$ & $--\infty=--$ & $=--\infty$ & $---0--=$ & $I=-$ & $=-\infty$ & $=---\infty$ & $I=$ & $m---\infty-\infty=-\infty$ & $=-\infty$ & $=-\infty$ & \\
\hline & PR 1 & I & 360 & 19 & 1 & I & 37 & 169 & 8 & & I & & ? & $I$ & & & & $I$ \\
\hline I & 2 & I & 280 & 15 & & I & 25 & 196 & 3 & & I & 1 & & T & & & & I \\
\hline & 3 & I & 257 & 2 & & I & 38 & 159 & 3 & & I & 1 & 3 & I & & & & I \\
\hline & 4 & I & 340 & 2 & & I & 54 & 108 & 3 & & I & & 5 & I & & & & I \\
\hline l & 5 & I & 143 & 1 & & I & 31 & 89 & 10 & & $I$ & & $?$ & $T$ & & & & I \\
\hline & 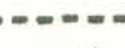 & $I$ & $-\infty-\infty$ & $--\infty-\infty$ & $=-$ & $\mathrm{I}-$ & $=-\infty=-$ & $-\infty-\infty-\infty$ & $m--\infty=$ & $-\infty-\infty-\infty$ & $I=-$ & $=$ & $-0--\infty$ & $I=$ & $-\infty-\infty-\infty--\infty$ & $-\infty=$ & $=-\infty$. & \\
\hline & 6 & I & 180 & 6 & & I & 26 & 95 & 7 & & $I$ & & 3 & $I$ & 8 & & & $I$ \\
\hline & 7 & I & 216 & 8 & & I & 28 & 108 & $B$ & & I & & 1 & I & 35 & & & I \\
\hline 1 & 8 & $T$ & 246 & 4 & 1 & I & 18 & 95 & 15 & & $I$ & h & ? & $T$ & 4 & & & I \\
\hline & 9 & I & ?.9? & 1 & 1 & I & 33 & 160 & 4 & & I & & 4 & $T$ & & & & I \\
\hline & 10 & $I$ & 315 & 5 & 1 & $\mathrm{I}$ & 25 & 156 & 4 & & $I$ & & 5 & t & & & & I \\
\hline & $---m-$ & $I$ & $-\infty-\infty=$ & $=---$ & $=-\infty$ & I $=$ & $=-\infty-\infty$ & $---\infty=-$ & $-\infty-\infty=$ & $---\infty-\infty$ & $I=-$ & $=-\infty$ & $-=-\infty-\infty$ & $\mathrm{I}=$ & $=-\infty-\infty=-\infty$ & $-\infty=$ & $-\infty-\infty$ & $-I$ \\
\hline 1. & 11 & I & 341 & R & & I & 37 & 169 & 16 & & I & 2 & 5 & $I$ & 14 & 36 & 2 & $I$ \\
\hline 1 & 12 & I & 188 & 9 & & I & 29 & 108 & 15 & & I & 1 & h & $\mathrm{T}$ & 2 & & & I \\
\hline [ & 13 & I & 154 & 4 & 2 & I & 33 & 127 & 16 & & l & $?$ & 4 & I & & & & I \\
\hline I & 14 & $I$ & 160 & 4 & & I & 23 & 104 & 19 & & $I$ & ? & a & $t$ & 6 & & & I \\
\hline I & 15 & $I$ & 148 & 8 & & $I$ & 29 & 106 & 20 & & I & 1 & 5 & $T$ & & & & $I$ \\
\hline & $=---$ & $I$ & $-0-\infty-\infty$ & $-=----$ & $=-\infty$ & $I-$ & $-=-\infty=0$ & $--=-\infty$ & $-\infty-\infty=-$ & $---\infty=-$ & $1=-$ & $=-\infty$ & $-\infty-\infty$ & $I=$ & $-\infty-\infty=-\infty-\infty=-\infty$ & $-\infty-$ & $=--=0$ & \\
\hline I & 16 & I & 317 & 6 & 1 & $I$ & 39 & 190 & 14 & & I & 3 & 1 & $I$ & 2 & & & I \\
\hline T & 17 & I & 282 & 1 & & I & 46 & 174 & 12 & & I & & 1 & T & & & & I \\
\hline I & 18 & $\mathrm{I}$ & 256 & 4 & & I & 46 & 146 & 8 & & I & 1 & 2 & $T$ & & 68 & & $I$ \\
\hline I & 19 & I & 278 & 5 & 2 & I & 36 & 1199 & 16 & & I & & 3 & I & & 41 & & I \\
\hline I & 20 & $I$ & 177 & & & I & 38 & 99 & 10 & & I & & 5 & $T$ & 6 & & & I \\
\hline & $=-\infty$ & $\mathrm{I}$ & $-\infty-\infty$ & $=\infty$ & & I - & $=-\infty-\infty$ & $=-m=-$ & $-\infty-\infty=$ & $-=---=$ & $=I=-$ & $-\infty$ & $-\infty=-\infty=$ & $I=$ & $-0 .-\infty=-\infty-\infty$ & $-\infty=$ & $=-\infty$ & \\
\hline I & 21 & $I$ & 176 & 5 & & I & 42 & 133 & 11 & & $I$ & 1 & 1 & $T$ & & & & I \\
\hline I & 22 & I & 345 & 4 & 4 & I & 34 & 142 & 8 & & I & & 3 & I & & 33 & 2 & I \\
\hline I & 23 & I & 252 & 1 & 4 & I & 48 & 156 & 18 & & I & & 4 & I & & 4 & & I \\
\hline T & 24 & I & 208 & 1 & 1 & I & 33 & 142 & 13 & & I & & 2 & I & & & & I \\
\hline I & 25 & I & 262 & 1 & 1 & $I$ & 36 & 156 & 7 & & $I$ & & 3 & $I$ & & 13 & & I \\
\hline & $-\infty-\infty$ & $I$ & $=-\infty$ & $---\infty$ & $-\infty$ & I - & $-\infty-\infty$ & $-\infty-\infty$ & $-0 .-10$ & $-0-\infty$ & $I=-$ & $-\infty$ & $-\infty-\infty$ & $I=$ & $-\infty-\infty-\infty-\infty-\infty$ & $=-\infty$ & $--\infty$ & \\
\hline I & 26 & I & 163 & & 2 & I & 27 & $9 ?$ & 6 & & $I$ & & & $T$ & & & & I \\
\hline I & 27 & I & 206 & 1 & 1 & I & 14 & 91 & 13 & & I & 4 & 7 & I & 33 & & & I \\
\hline$I$ & 28 & I & 187 & 3 & & I & 45 & 138 & 11 & & I & & & I & & & & I \\
\hline$\tau$ & 29 & I & 167 & 4 & & I & 12 & 83 & 18 & & I & 5 & & $T$ & 3 & & & I \\
\hline I & 30 & $I$ & 355 & 20 & 1 & $\mathrm{~T}$ & 33 & 165 & 17 & & $\mathrm{I}$ & 1 & 5 & $I$ & & & & I \\
\hline & $-\infty-\infty$ & $\mathrm{I}$ & $=---\infty$ & $--\infty-\infty$ & $-m-$ & $I=$ & $=-\infty=$ & $0-0-\infty$ & $-0-0-\infty$ & $-=-\infty-\infty$ & $I=-$ & $-\infty$ & $--\infty=-0$ & $I=$ & $---0=--\infty-\infty$ & $-=-$ & $=-\infty$ & \\
\hline & AAY 1 & I & 435 & 19 & 6 & I & 29 & 155 & 10 & & $I$ & 2 & & $\mathrm{I}$ & 10 & & & I \\
\hline I & 2 & I & 477 & 6 & 1 & I & 34 & $1 \Delta a$ & 9 & & $I$ & & ? & I & & & & I \\
\hline$I$ & 3 & I & 268 & 12 & & $T$ & 26 & 152 & 15 & & $I$ & & & $\mathrm{~T}$ & 6 & & & I \\
\hline I & 4 & I & 228 & 7 & 1 & I & 31 & 158 & 22 & & I & 1 & 2 & $?$ & 4 & & & I \\
\hline T & 5 & I & 234 & 6 & & I & 26 & 114 & 29 & & I & & 2 & I & & & & I \\
\hline & $-=-\infty$ & $\mathrm{I}$ & $-0-\infty=-$ & $-\infty-\infty$ & & $I=$ & $\infty=-\infty$ & $-\infty-\infty$ & $=-\infty-\infty$ & $=-\infty=-\infty$ & $J=-$ & $-\infty$ & $-=-\infty=-m$ & $I=$ & $-\infty+\infty-\infty-\infty$ & $=-\infty$ & $=--=0$ & \\
\hline I & 6 & I & 221 & 1 & & I & 28 & 132 & 15 & & I & 1 & 4 & $T$ & 26 & & 1 & I \\
\hline I & 7 & I & 2.39 & 4 & & I & 32 & 149 & 20 & & I & 1 & & $T$ & & & 3 & I \\
\hline I & $B$ & I & 231 & & 2 & I & 43 & 117 & 5 & & I & & 2 & T & & & & I \\
\hline T & 9 & $\mathrm{I}$ & 90 & 1 & & $\mathrm{~J}$ & 16 & 79 & 4 & & I & & & $T$ & & & & I \\
\hline$T$ & 10 & I & 174 & & & 1 & 22 & 135 & 19 & & $I$ & & & $T$ & 9 & & & I \\
\hline & $-\infty-\infty$ & $\mathrm{I}$. & $-\infty-\infty-\infty$ & $-\infty$ & & - I - & $=-\infty-\infty$ & $-\infty=-\infty$ & $--\infty-\infty$ & $-0-\infty-\infty$ & $-I=-$ & & $-m=-$ & $I=$ & $m-\infty=-\infty-\infty$ & $=-\infty$ & $=-\infty$ & \\
\hline$I$ & 11 & I & 254 & 7 & 1 & $I$ & 37 & 150 & 5 & & 1 & 1 & & 1 & 5 & & & I \\
\hline I & 12 & I & 264 & 7 & & I & 27 & 170 & 14 & & I & 2 & $?$ & I & & & & I \\
\hline I & 13 & I & 359 & 1 & & I & 28 & 143 & 13 & & $I$ & & 5 & $T$ & & & & I \\
\hline I & 14 & I & 163 & 3 & & $\mathrm{~J}$ & 32 & 113 & 7 & & I & 2 & 3 & 1 & 5 & & & I \\
\hline I & 15 & I & 407 & ?. & 4 & I & 24 & 155 & ? & & I & & 1 & $T$ & 9 & 11 & 2 & $I$ \\
\hline
\end{tabular}




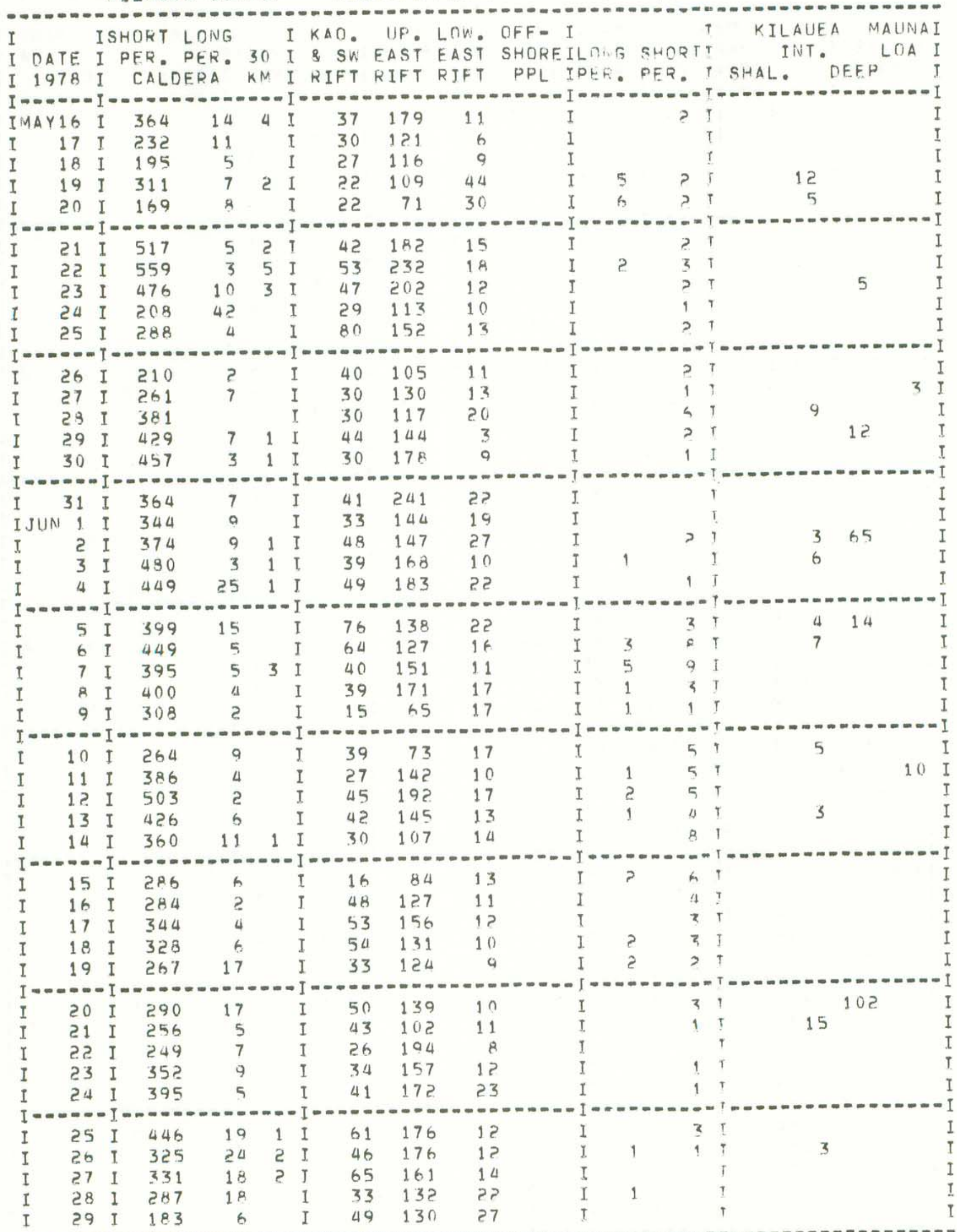




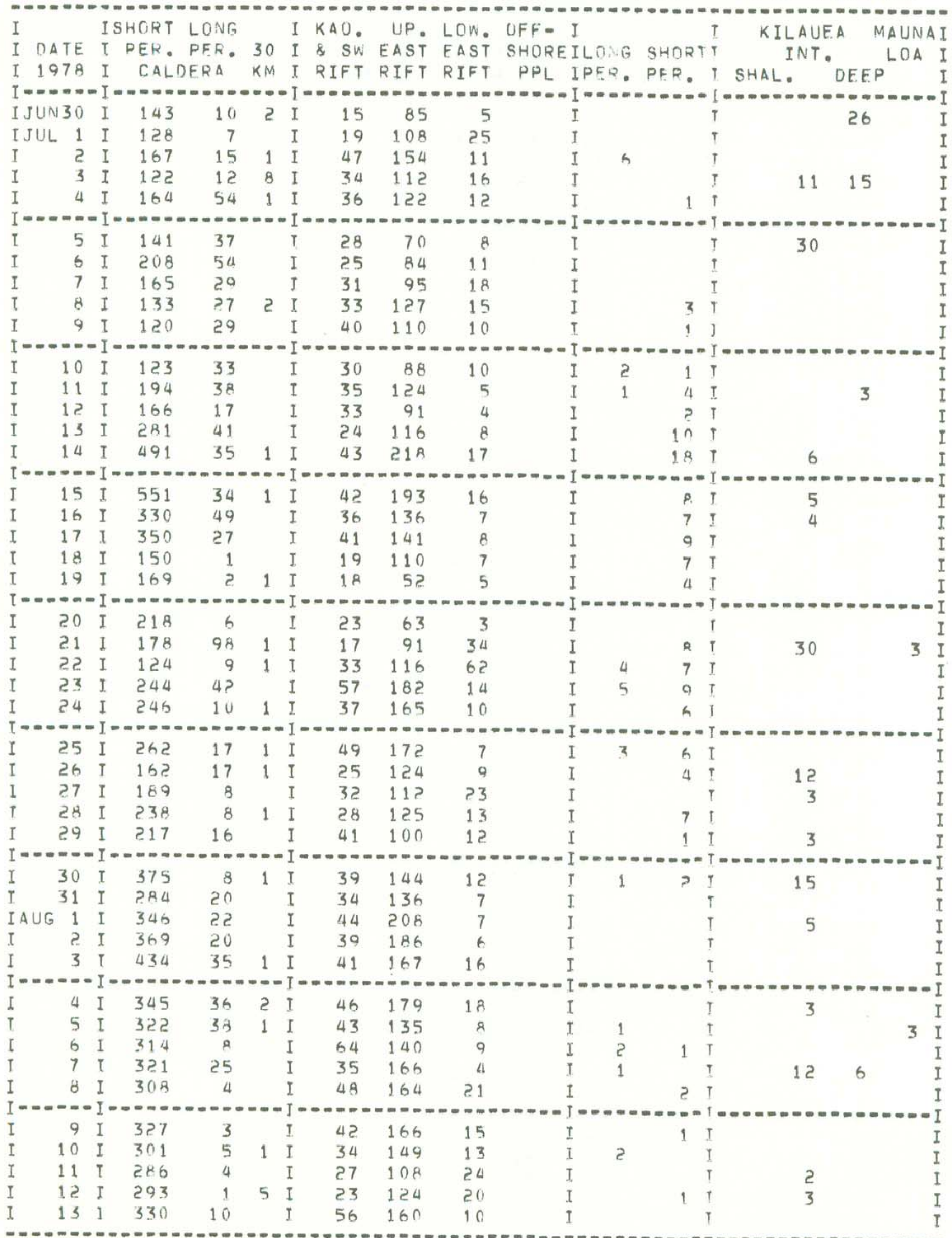




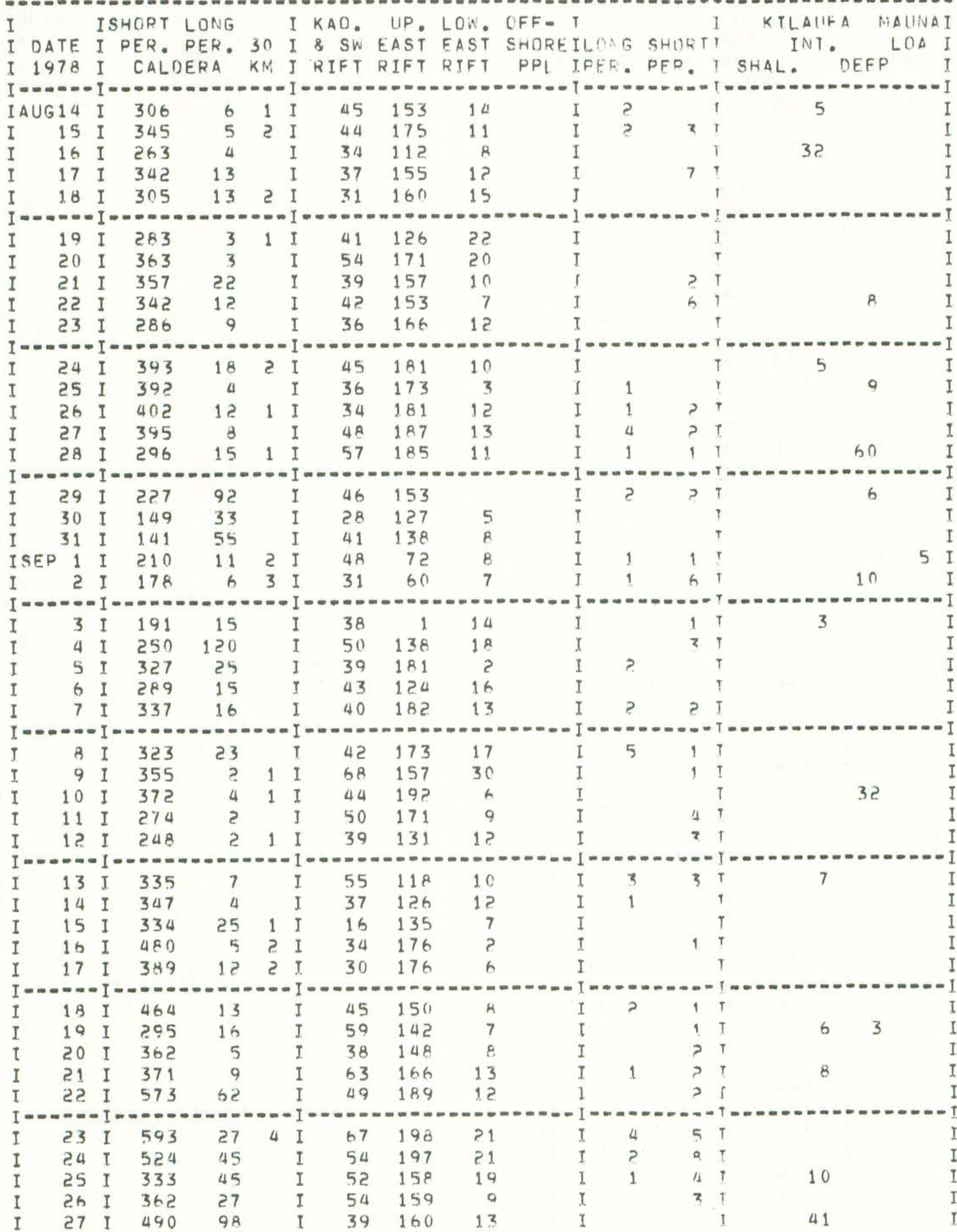




\begin{tabular}{|c|c|c|c|c|c|c|c|c|c|c|c|c|c|c|c|c|}
\hline I & \multicolumn{2}{|c|}{ ISHORT } & \multicolumn{2}{|l|}{ LONG } & \multicolumn{2}{|c|}{$I K A O$. } & UP. & LOW. & UFF - & \multicolumn{2}{|l|}{ I } & \multicolumn{2}{|r|}{ I } & KILAUIEA & \multicolumn{2}{|c|}{ MAUNAI } \\
\hline DATE & I & PER. & $P E R$. & 30 & I & $\& S W$ & FAST & EAST & SHORF & ILO & Nis & SHIRT & & INT. & & LOA I \\
\hline $197 \%$ & I & CALD & ERA & Kin & I & RIF T & R IF T & RIF T & PPL & IPE & E. & DFD. & $T$ & SHAL. $\quad D$ & DEEP & $T$ \\
\hline \multicolumn{17}{|c|}{ 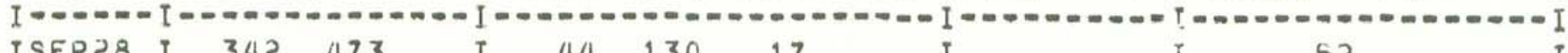 } \\
\hline SEP 8 & I & 342 & 473 & & I & 44 & 130 & 17 & & I & & & I & & & \\
\hline ?9 & I & $2 ? 8$ & 69 & 2 & I & 27 & 43 & 25 & & I & & $?$ & I & 5 & & \\
\hline 30 & I & 307 & 111 & & I & 28 & 61 & 17 & & I & & & T & 25 & & \\
\hline DCT 1 & I & 416 & 63 & 2 & I & 37 & 139 & 8 & & I & & ? & r & & & \\
\hline$a$ & I & 398 & 35 & & $\tau$ & 45 & 150 & 6 & & I & & 1 & T & & & \\
\hline & I & 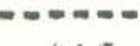 & & - & $I$ & $--\infty$ & $-\infty-\infty$ & $-\infty$ & $-\pi$ & $I=$ & & $-0-\infty-\infty-\infty$ & & $-\infty-\infty=-\infty-\infty-\infty$ & $-=-\infty$ & $\cdots-\infty$ \\
\hline 3 & I & 415 & 23 & 1 & I & 52 & 184 & 6 & & I & & & $\uparrow$ & & & \\
\hline 4 & I & 343 & 15 & 1 & I & 60 & 168 & 8 & & J & 4 & 3 & $T$ & & & \\
\hline 5 & I & 481. & 9 & & I & 40 & 143 & 11 & & T & 2 & 5 & $T$ & & & \\
\hline 6 & I & 457 & 3 & & I & 54 & 139 & 14 & & I & & $?$ & I & & & \\
\hline 7 & I & 460 & 22 & 1 & I & 45 & 174 & 19 & & I & & & I & & & \\
\hline$-\infty$ & I & $--\infty-\infty$ & $=-\infty$ & & I & $--\infty$ & $=-\infty$ & 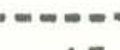 & $-\infty-\infty=-\infty$ & $I=-$ & $=-\infty$ & $-\infty-\infty-\infty$ & & $\Rightarrow-\infty---\infty=0$ & $-\infty-\infty$ & $-\infty-\infty$ \\
\hline 8 & I & 365 & 60 & & I & $\Delta 1$ & 140 & 15 & & I & & ? & $T$ & 7 & & \\
\hline c & I & 359 & 27 & & I & 45 & 142 & 9 & & I & 1 & & I & & & \\
\hline 10 & I & 388 & 14 & & I & 65 & 197 & Q & & I & & & $\tau$ & & & \\
\hline 11 & I & 351 & 118 & & I & 31 & 140 & 7 & & I & 3 & 1 & i & & & \\
\hline 12 & I & 340 & 66 & ? & I & 60 & 175 & 8 & & I & & 1 & I & 44 & & \\
\hline 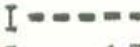 & 1 & $--\infty-\infty .-\infty$ & $-\infty$ & 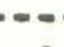 & I. & $=-\infty$ & $=-\infty$ & $-\infty$ & $-\infty$ & $I=$ & $-\infty$ & $-0 .-0$ & & 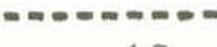 & $--\infty-\infty$ & $--\infty-1$ \\
\hline 13 & I & 284 & 93 & 2 & I & 51 & 81 & 16 & & I & 3 & & $\mathrm{~T}$ & 62 & 28 & \\
\hline 14 & I & 438 & 50 & 5 & I & 36 & 60 & 24 & & โ & 5 & & $T$ & 4 & & \\
\hline 15 & I & 318 & 5 & & I & 31 & 154 & 13 & & I & & & I & 6 & 2 & \\
\hline 16 & I & 311 & 10 & & I & 35 & 144 & 13 & & I & & 1 & $T$ & 3 & 12 & \\
\hline 17 & I & 263 & 12 & & I & 49 & 167 & 18 & & I & ? & 3 & r & & 13 & \\
\hline-0 & 1 & $--\infty$ & $\infty$ & & $I$ & $=-\infty$ & $=-\infty$ & $-\infty$ & & $I-$ & $-\infty$ & $-\infty-\infty$ & & $--0-0-0.0-0$ & $-\infty-\infty$ & $--\infty-1$ \\
\hline 18 & I & 295 & 20 & & I & 56 & 124 & 7 & & I & 1 & & $!$ & & & \\
\hline 19 & I & 353 & 14 & & I & 60 & 130 & ? & & I & ? & & $\mathrm{T}$ & 4 & & \\
\hline 20 & I & 2.24 & 9 & & I & 77 & 186 & 13 & & I & 1 & 1 & 1 & & & \\
\hline 21 & I & 201 & 5 & & 1 & $4 n$ & 127 & 11 & & I & & & 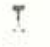 & & & . \\
\hline 22 & I & 354 & 12 & & I & 50 & 143 & 11 & & I & & & $T$ & & & \\
\hline$-\infty$ & 1 & $---\infty=0$ & $-\infty$ & - & $\mathrm{I}$ & $=-$ & $=-\infty$ & $-\infty=$ & & I - & $-\infty$ & $-\infty-\infty$ & & $=--m-\infty-\infty-\infty$ & $-\infty-\infty$ & $-\ldots-I$ \\
\hline 23 & I & 146 & 5 & 1 & I & 32 & 137 & 18 & & I & 1 & & t & & & \\
\hline 24 & I & 254 & 1 & 1 & I & 40 & 148 & 14 & & I & 2 & 2 & $T$ & & & \\
\hline 25 & I & 154 & 14 & 3 & I & 50 & 140 & 9 & & I & ? & 1 & I & & & \\
\hline 26 & I & 190 & 9 & 2 & I & 54 & 136 & 6 & & I & 1 & 1 & $?$ & 20 & & t \\
\hline 27 & I & 157 & 5 & & I & 30 & 140 & 13 & & I & 3 & 1 & $T$ & & & 1 \\
\hline$--\infty$ & I & $=--x-0$ & & $+\infty$ & I & $=-\infty$ & $=-\infty$ & $-\infty$ & 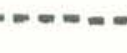 & $I=-$ & & $--=--=$ & $I=$ & $-\infty-\infty$ & $-\infty-\infty$ & $-\infty-1$ \\
\hline 28 & I & 160 & 5 & & I & 53 & 144 & 17 & & I & & 1 & $\mathrm{i}$ & & & 8 \\
\hline 29 & I & 123 & 3 & 1 & I & 51 & 179 & 9 & & I & & $?$ & I & 4 & & J \\
\hline 30 & I & 101 & 3 & 2 & I & 57 & 117 & 21 & & I & & 1 & T & 6 & & \\
\hline 31 & I & 211 & 8 & & I & 68 & 114 & 9 & & I & 12 & is & $\mathrm{T}$ & & & 2 \\
\hline I NOV 1 & I & 113 & 14 & 1 & I & 39 & 112 & 10 & & I & 3 & 34 & $T$ & & & \\
\hline$-=-x$ & & $---\infty$ & & & I. & $-\infty$ & $=-$ & s. & $0-\infty$ & $I=-$ & $=-1$ & 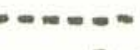 & to & $-\infty$ & $=--$ & $=--1$ \\
\hline$a$ & 1 & 273 & 14 & & I & 48 & 142 & 8 & & I & 18 & 2 & $\tau$ & & & 1 \\
\hline 3 & T & 157 & 1 & 1 & I & 17 & 43 & 46 & & $I$ & 28 & 3 & $\mathrm{t}$ & & 10 & 5 \\
\hline 4 & I & 87 & 2 & & I & 29 & 57 & 35 & & I & Q & 1 & T & & & \\
\hline 5 & T & 153 & 6 & & I & 2.2 & 113 & 9 & & I & 21 & & $T$ & 4 & & 1 \\
\hline 6 & I & 47 & 1 & 1 & I & 13 & 86 & 7 & & I & 2 & & $T$ & & & I \\
\hline & & 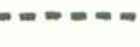 & & 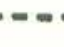 & 10 & & 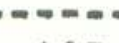 & &.$--=-$ & $l=-$ & $-\infty$ & $-0-0=-$ & & & $=-$ & $=-\infty$ \\
\hline 7 & I & & 1 & & I & 23 & 103 & 27 & & I & 36 & 3 & $T$ & & & \\
\hline 8 & I & 69 & & & I & 42 & 115 & 18 & & I & 41 & & $\stackrel{T}{1}$ & & & \\
\hline ? & I & 51 & 2 & 1 & I & 49 & 115 & 13 & & I & 23 & & ? & & & \\
\hline 10 & I & 39 & 8 & 1 & I & 45 & 111 & 25 & & I & 6 & 3 & $\tau$ & & & \\
\hline 11 & I & 32 & 1 & & I & 51 & 69 & in & & I & 10 & 1 & $T$ & & 96 & \\
\hline
\end{tabular}


I ISHORT LONG I KAO. UP. LOW. OFF - I

I DATE I PER PER 30 I \& SW EAST EAST SHOREILONOS SHORT

I 1978 I CALDERA KM I RIFT RIFT RIFT PPL TPFR. PFR, T SHAL. DEEP I

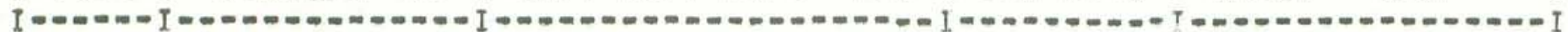
INOV15I 53

I 13 I

I 14 I

I $15 \mathrm{I}$

I 16 I

$I=-\infty=-10$

I

I

I

I I

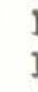
I

$$
\text { I }
$$

$$
\text { I }
$$

\section{I}

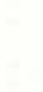




\section{KILAUEA SUMMIT KILAUEA FLANIK}

MALINA LOCA

TREMOR (MINUTES)

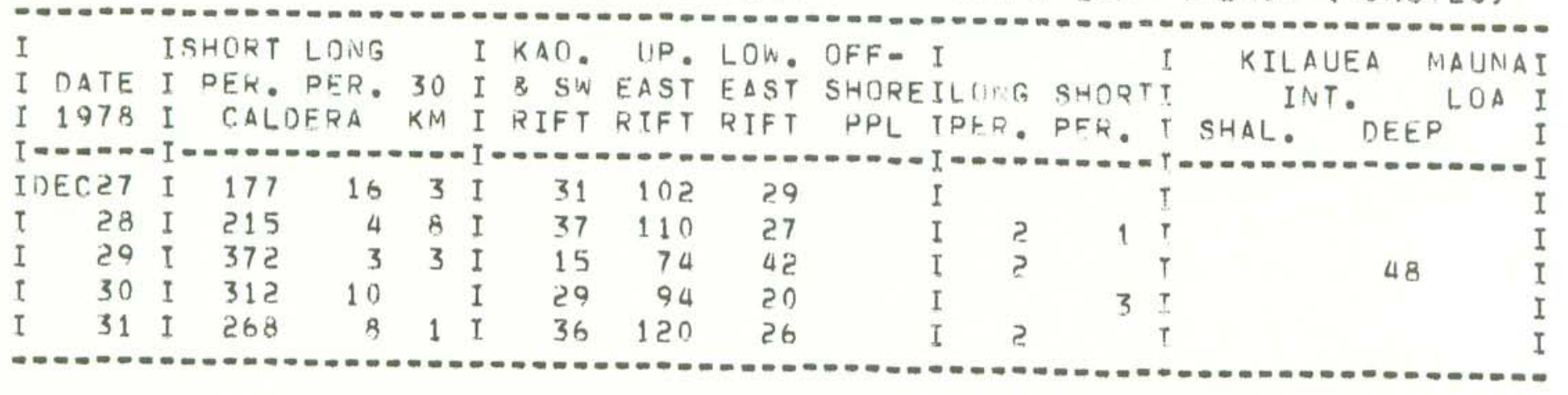




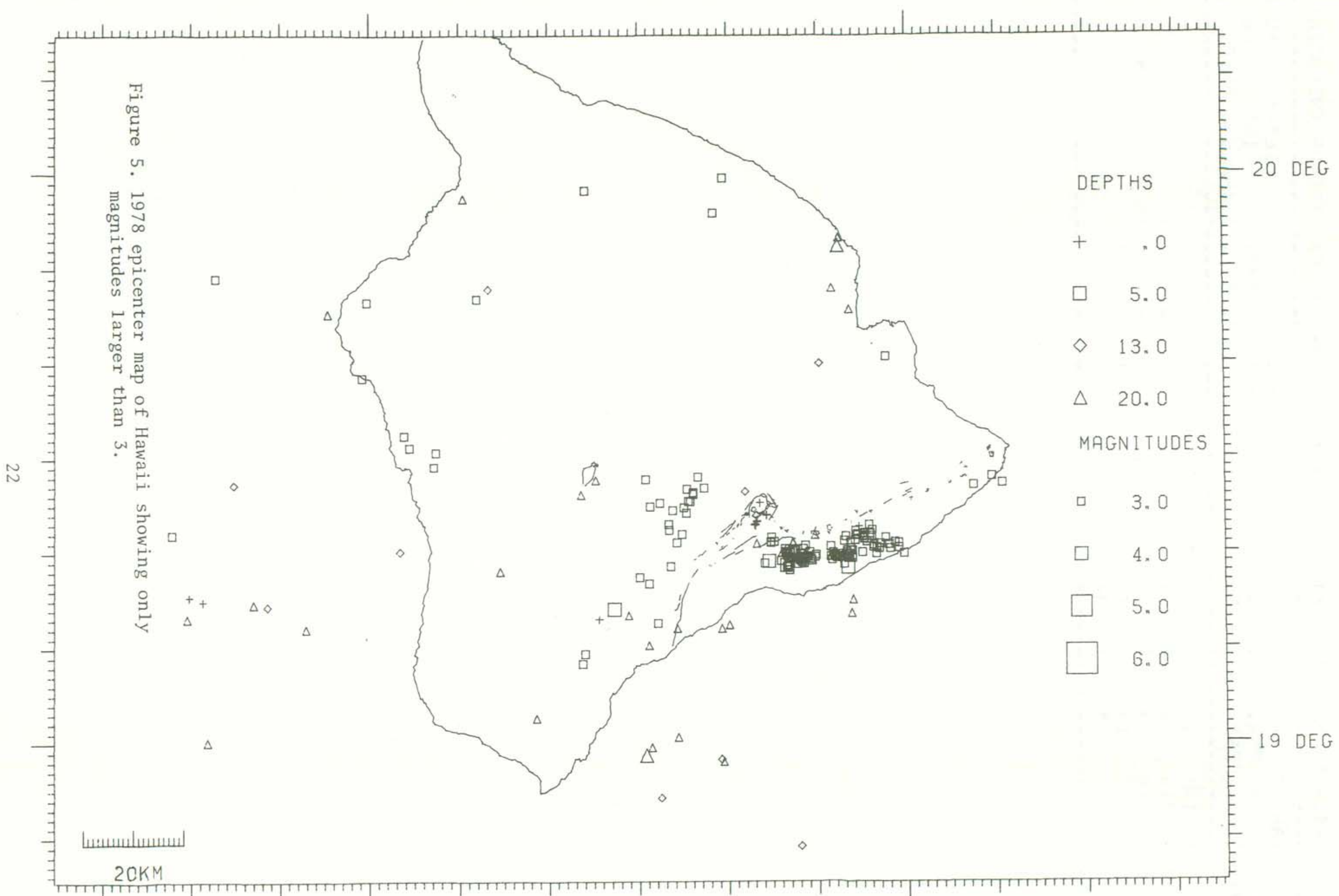

20KM

156 DEG

155 DEG 


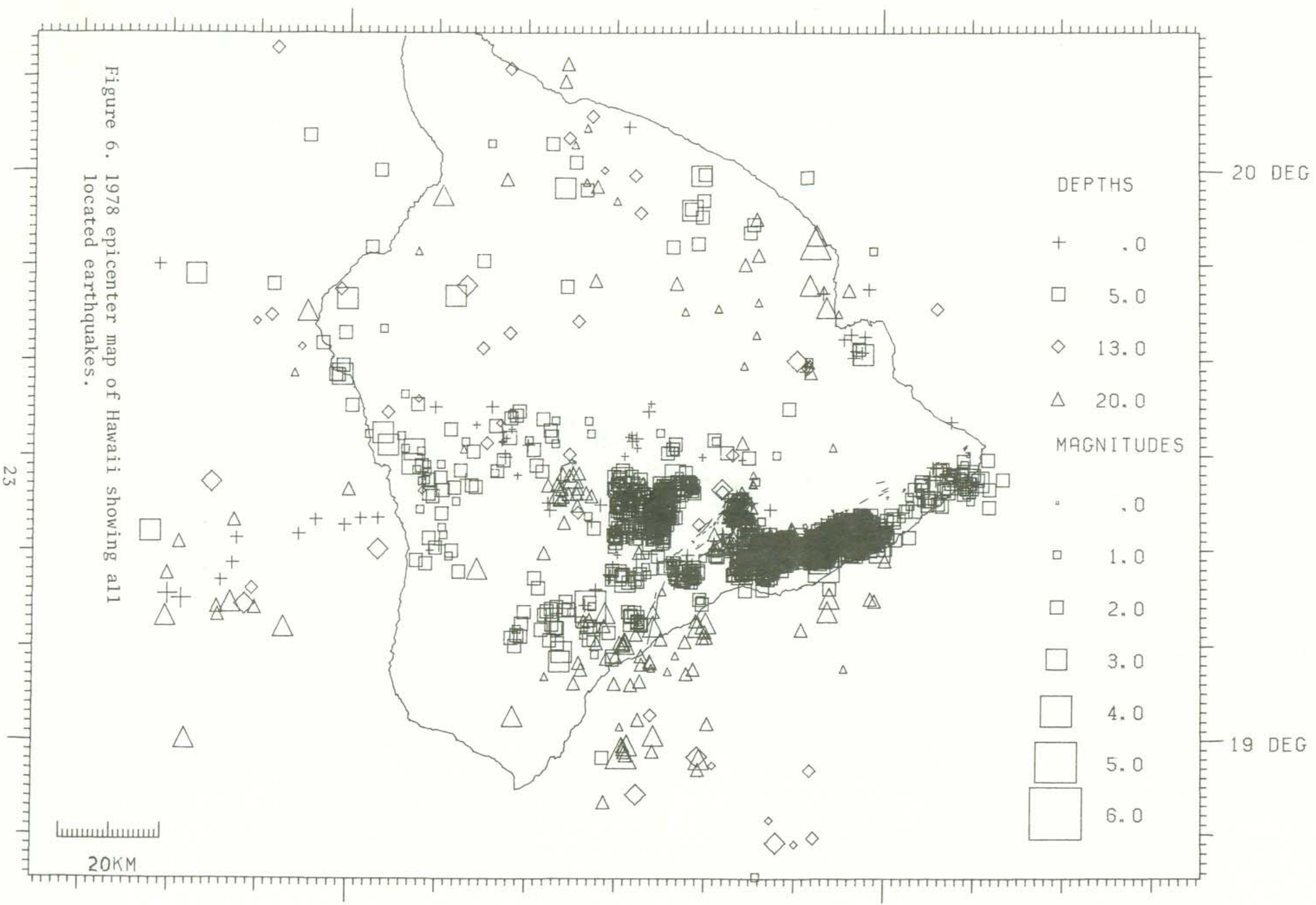




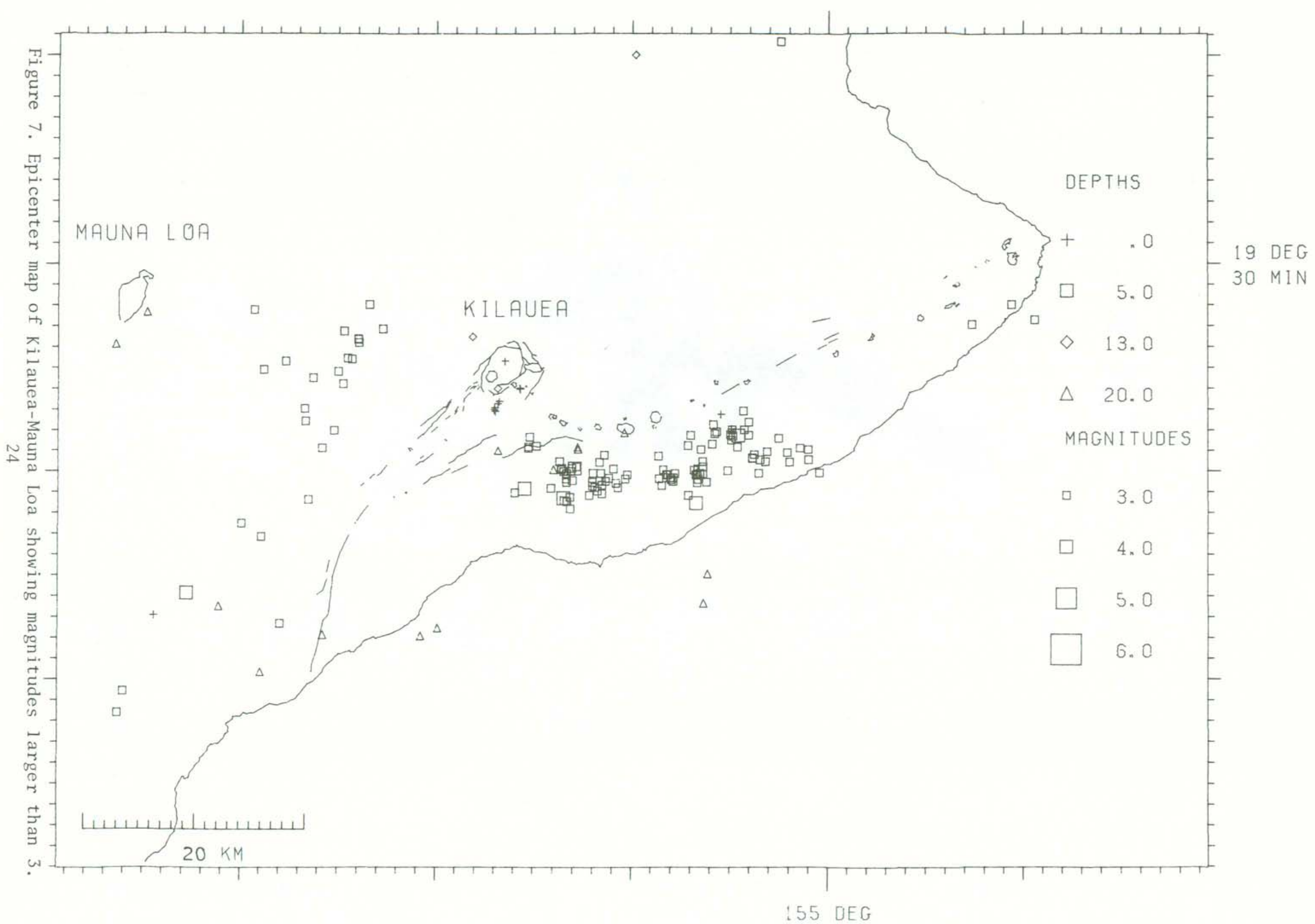




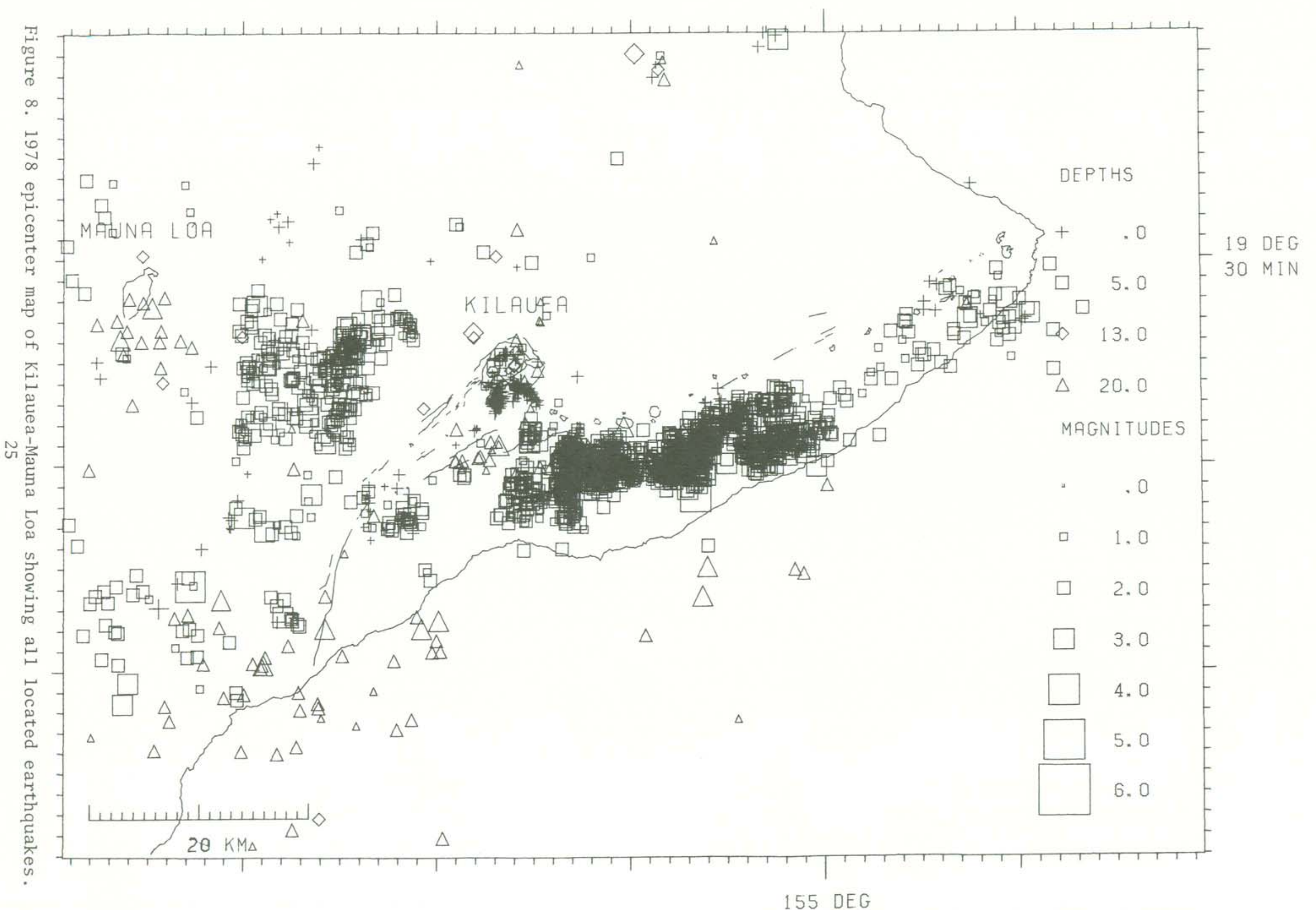




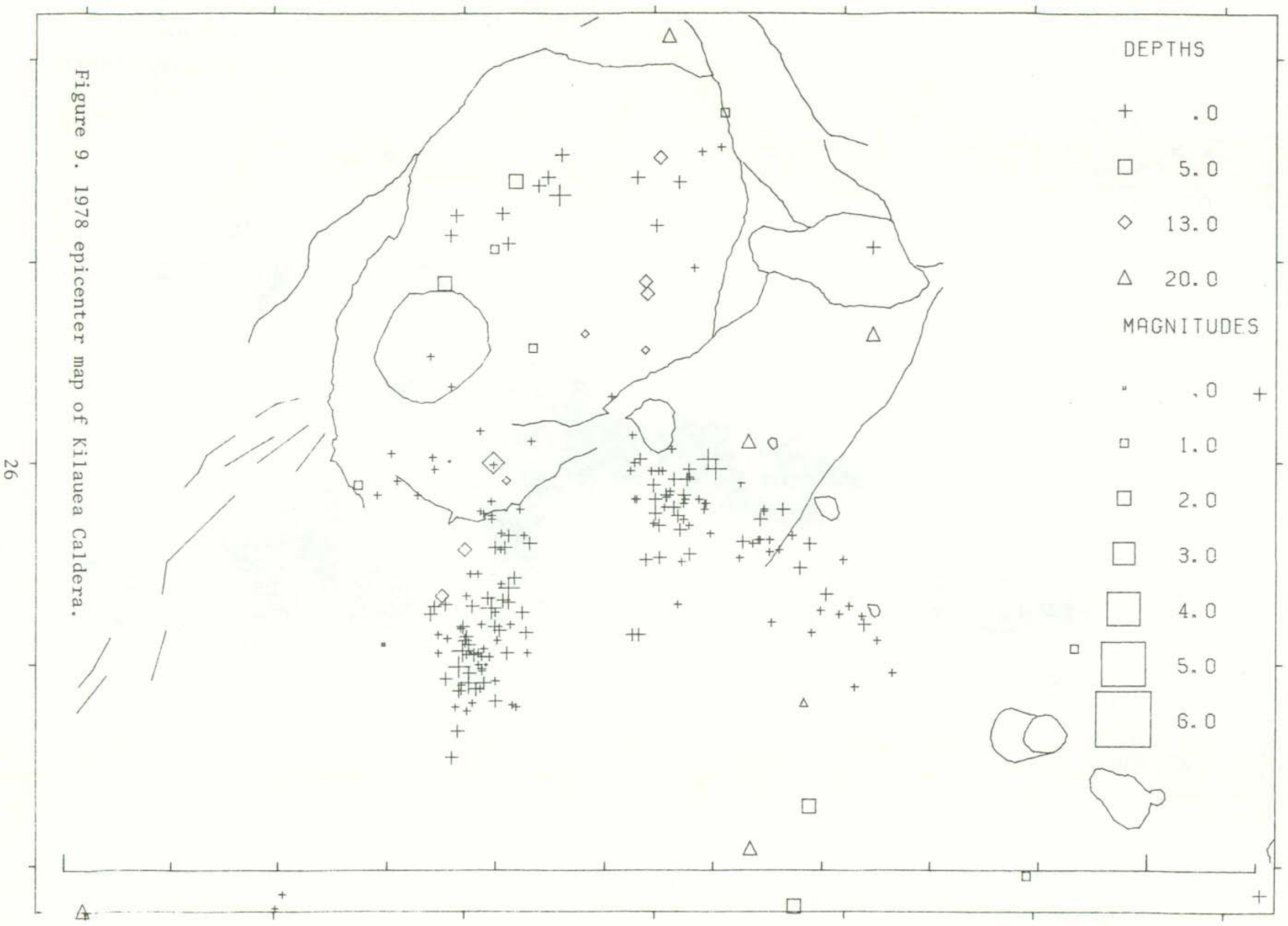


Table 4. Coordinates of named regions.

\begin{tabular}{|c|c|c|c|c|c|c|c|c|c|c|}
\hline \multirow{2}{*}{ Name } & \multicolumn{2}{|c|}{ NORTH } & \multicolumn{2}{|c|}{ SOUTH } & \multicolumn{2}{|c|}{ EAST } & \multicolumn{2}{|c|}{ WEST } & \multirow{2}{*}{\multicolumn{2}{|c|}{$\begin{array}{c}\text { DEPTHS } \\
\mathrm{km}\end{array}$}} \\
\hline & $\mathrm{D}$ & M & $\mathrm{D}$ & M & $\mathrm{D}$ & M & $\mathrm{D}$ & M & & \\
\hline SPC & 19 & 27 & 19 & 23 & 155 & 15 & 155 & 19 & 0 & -5.5 \\
\hline LPC & 19 & 27 & 19 & 23 & 155 & 15 & 155 & 19 & 5.5 & -11 \\
\hline INT & 19 & 29 & 19 & 18 & 155 & 22 & 155 & 12 & 11 & -22 \\
\hline DEP & 19 & 29 & 19 & 18 & 155 & 22 & 155 & 12 & 22 & -70 \\
\hline UER & 19 & 23 & 19 & 19 & 155 & 6 & 155 & 15 & & \\
\hline $\mathrm{K} \cap \mathrm{A}$ & 19 & 23 & 19 & 17 & 155 & 15 & 155 & 19 & & \\
\hline SWR & 19 & 22 & 19 & 16 & 155 & 19 & 155 & 25 & & \\
\hline IIKF & 19 & 29 & 19 & 27 & 155 & 19 & 155 & 30 & & \\
\hline MF.R & 19 & 25 & 19 & 16 & 155 & 1 & 155 & 6 & & \\
\hline LER & 19 & 31 & 19 & 20 & 154 & 47 & 155 & 1 & & \\
\hline POL & 19 & 19 & 19 & 10 & 155 & 6 & 105 & 15 & & \\
\hline LSW & 19 & 16 & 19 & 0 & 155 & 21 & 155 & 34 & & \\
\hline PPL & 19 & 10 & 18 & 30 & 155 & 0 & 155 & 21 & & \\
\hline HLP & 19 & 17 & 19 & 10 & 155 & 15 & 155 & 21 & & \\
\hline MOK & 19 & 40 & 19 & 22 & 155 & 30 & 155 & 45 & & \\
\hline GLN & 19 & 31 & 19 & 23 & 155 & 1 & 155 & 19 & & \\
\hline KON & 19 & 55 & 19 & 15 & 155 & 45 & 156 & 10 & & \\
\hline HEA & 19 & 22 & 19 & 0 & 155 & 25 & 155 & 45 & & \\
\hline $\mathrm{KOH}$ & 20 & 15 & 19 & 55 & 155 & 30 & 156 & 0 & & \\
\hline NER & 19 & 40 & 19 & 29 & 155 & 12 & 155 & 30 & & \\
\hline HIL & 19 & 50 & 19 & 31 & 154 & 47 & 155 & 12 & & \\
\hline KKU & 20 & 5 & 19 & 40 & 155 & 0 & 155 & 45 & & \\
\hline DIS & F.VF & PLA & ELSE & & & & & & & \\
\hline
\end{tabular}

When coordinates imblv an overlap, precedence is given as shown in Figure 2. 
Table 5 is a chronological listing of successfully located earthquakes. For each event the following data are presented:

Origin time in Hawaiian Standard Time: date, hour (HR), minute (MN), and second $(\mathrm{SEC})$.

Epicenter in degrees and minutes of north latitude (LAT N) and west longitude (LON W).

DEPTH - Depth of focus in $\mathrm{km}$.

AMP MAG - Amplitude magnitude, if determined.

DUR MAG - Duration magnitude, if determined.

NR - Number of arrivals (P or S) used for solution.

NS - Number of S arrivals used for solution.

GAP DEG - Largest azimuthal separation in degrees between stations.

RMS SEC - Root mean square error of time residuals in sec.

$$
\mathrm{RMS}=\left(\sum \mathrm{R}_{i}^{2} / \mathrm{NR}\right)^{1 / 2}
$$

MIN DIS - Epicentral distance in $\mathrm{km}$ to the third nearest station.

ERH $\mathrm{km}$ - Standard error of the epicenter in $\mathrm{km}$.

ERZ $\mathrm{km}$ - Standard error of depth of focus in $\mathrm{km}$.

REMK - Remarks, three letter code for geographic location of event. See Figure 2 for location of mnemonic code. Additional one letter codes have the following meanings:

F - felt

L - long period character

T - associated with harmonic tremor

B - quarry or other blast

* - the location program had a convergence problem, which usually means that the depth may be unreliable.

Table 5 lists all events located during 1978. Table 6 lists only events of magnitude 3.0 or larger. 
ORIGIN TIME LATN LON W DEPTH AMP OHR GAP RMS MIN ERH ERZ NO YEAR MON OA HRMN SEC DEG MIN DEG MIN KM MAE MAG NR NS OEG SEC OIS KM KM FM REMK $\begin{array}{lllllllll}1978 \text { JAN } & 2 & 8 & 2 & 38.01 & 19 & 21.11 & 155 & 1.21 \\ 2 & 1234 & 23.54 & 19 & 17.56 & 155 & 15.78\end{array}$ 2 $162913.751927 .10 \quad 15522.55$

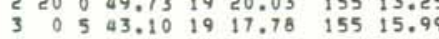
$\begin{array}{llllllll}3 & 127 & 59.15 & 19 & 19.02 & 155 & 12.93 \\ 3 & 136 & 8.06 & 19 & 23.16 & 155 & 2.26\end{array}$ 3 2 $6 \begin{array}{lllll}16.39 & 19 & 19.09 & 155 & 10.6\end{array}$ $\begin{array}{rrrrrrr}3 & 730 & 2.34 & 19 & 21.45 & 155 & 24.68 \\ 3 & 1031 & 12.80 & 19 & 20.40 & 155 & 7.18\end{array}$ $\begin{array}{rrrrrrrr}3 & 16 & 6 & 8.41 & 19 & 20.03 & 155 & 11.78 \\ 3 & 1857 & 18.65 & 19 & 21.55 & 155 & 2.58\end{array}$ 4. $842 \quad 27.33 \quad 19 \quad 19.43 \quad 155 \quad 10.83$ $4846 \quad 11.77$ i9 20.65 155 11.26

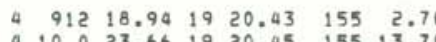
4 1 $10023.661920 .45 \quad 15513.70$ $41137 \quad 37.99$ is $58.25 \quad 155 \quad 31.41$ $4114231.15 \quad 1920.65 \quad 155 \quad 6.92$ a $1343 \quad 31.51 \quad 1920.38 \quad 155 \quad 13.52$

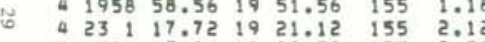
$\begin{array}{rrrrrrr}5 & 353 & 13.26 & 19 & 20.30 & 155 & 9.52 \\ 5 & 358 & 46.70 & 19 & 18.96 & 155 & 15.97\end{array}$ $\begin{array}{lllllll}5 & 1213 & 56.02 & 19 & 19.03 & 154 & 59.91\end{array}$ 5 1529 $16.431922 .81 \quad 15525.16$

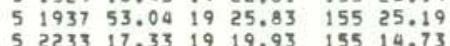

$5 \quad 2255 \quad 23.87 \quad 19 \quad 20.83 \quad 155 \quad 10.53$

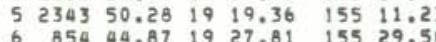
$\begin{array}{lllllll}6 & 1033 & 23.56 & 19 & 10.39 & 155 & 32.11 \\ 6 & 1755 & 48.98 & 19 & 21.72 & 155 & 5.80\end{array}$

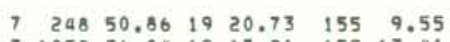

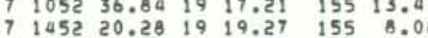
$\begin{array}{lllllll}7 & 1455 & 11.32 & 19 & 20.29 & 155 & 6.10 \\ 7 & 19 & 96.40 & 19 & 27.89 & 154 & 54.91\end{array}$ $72030 \quad 35.92 \quad 1959.60 \quad 155 \quad 20.50$ 721
7 $257.43 \quad 1925.61 \quad 15524.86$ $\begin{array}{lllllll}7 & 2235 & 1.51 & 19 & 19.74 & 155 & 10.25\end{array}$

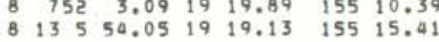

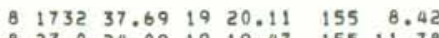

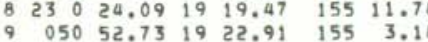

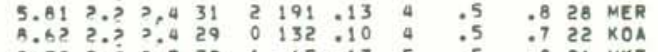

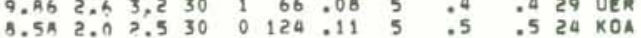

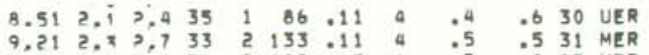

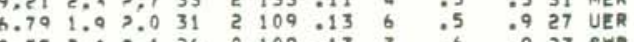
$\begin{array}{llllllllllll}8.77 & 2.0 & 7.1 & 26 & 0 & 109 & 13 & 3 & .6 & .9 & 23 & \text { SWR } \\ 7.4 h & 2.7 & 7.6 & 18 & 2 & 199 & 08 & 6 & .7 & 1.4 & 14 & \text { UER }\end{array}$

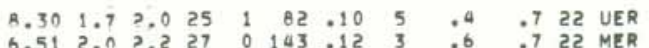

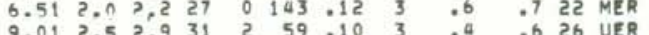

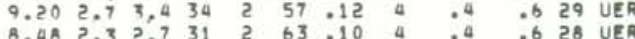
$12.71 \quad 2.52 .334 \quad 25262.1943 \quad 2.0 \quad 3.3330$ OIS

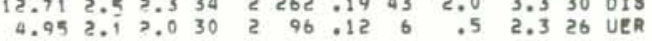
$\begin{array}{llllllllllllll}9.76 & 2.5 & 2.7 & 33 & 2 & 66 & .11 & 4 & .4 & .5 & 28 & \text { UER }\end{array}$

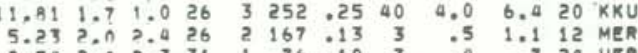

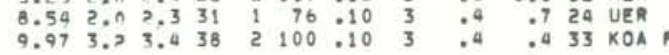
$\begin{array}{llllllllllll}44.07 & 2.4 & 3.0 & 34 & 2 & 220 & .10 & 6 & 1.1 & 1.9 & 29 & 018\end{array}$

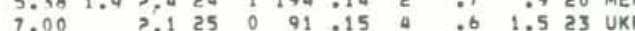

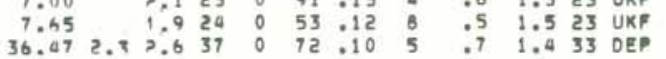
$\begin{array}{llllllllllll}7.89 & 2.0 & 2.1 & 29 & 0 & 72 & 110 & 3 & .4 & .7 & 27 & \text { UER }\end{array}$

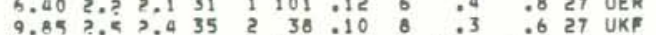

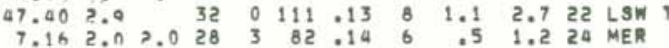
$6.901 .91 .928 \quad 2 \quad 69.11 \quad 3 \quad .451 .025$ UER

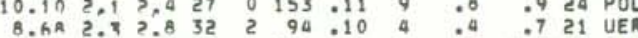

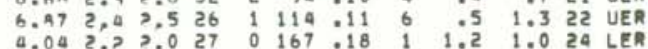
$\begin{array}{llllllllllll}9.71 & 2.0 & \times .2 & 33 & 2 & 274 & .13 & 27 & 1.9 & .8 & 28 & \mathrm{KKU} F\end{array}$

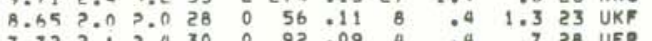

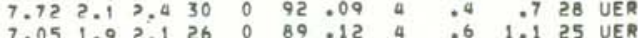

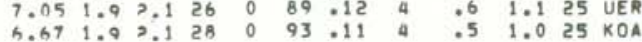
$\begin{array}{llllllllllll}7.59 & 2.7 & 2.6 & 32 & 0 & 79 & .10 & 4 & .5 & .7 & 31 & \text { UER }\end{array}$

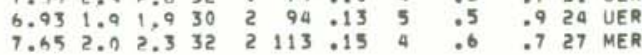

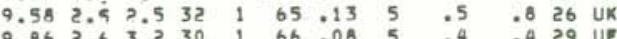

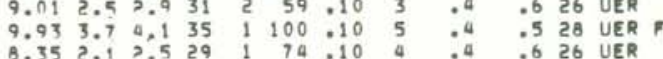
$\begin{array}{llllllllllll}5.59 & 2.3 & 2.1 & 28 & 2 & 159 & .13 & 1 & .5 & 1.0 & 23 & \text { MER }\end{array}$
ORIGIN TIME LATN LON W TEA MON OA HRMN SEC DEG MIN DEG MIN 1978 JAN $91127 \quad 16.211919 .44 \quad 155$ 9.31

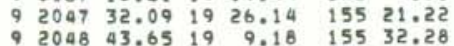
$\begin{array}{rrrrrrr}9048 & 43.65 & 19 & 9.18 & 155 & 32.28 \\ 10 & 352 & 19.23 & 19 & 27.94 & 154 & 51.33\end{array}$ $\begin{array}{lllllll}10 & 140 & 7.29 & 19 & 18.96 & 155 & 6.85\end{array}$ $\begin{array}{llrllll}10 & 140 & 7.29 & 19 & 18.96 & 155 & 6.85 \\ 10 & 1440 & 57.30 & 19 & 20.67 & 155 & 4.35\end{array}$

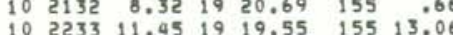
$102238 \quad 4.02 \quad 1920.13 \quad 155 \quad 12.54$

$\begin{array}{llllllll}10 & 23 & 9 & 54.40 & 19 & 19.63 & 155 & 13.37\end{array}$

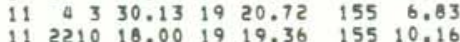
$12 \quad 63529.841919 .64 \quad 15599.70$ $\begin{array}{lllllll}12 & 2047 & 49.03 & 19 & 19.63 & 155 & 11.28\end{array}$

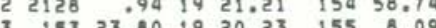
$\begin{array}{llllllll}13 & 1040 & 10.72 & 19 & 18.98 & 155 & 12.25\end{array}$ $\begin{array}{lllllll}13 & 1240 & 2.27 & 19 & 20.78 & 155 & 1.90\end{array}$

$\begin{array}{llllllll}13 & 15 & 15.11 & 19 & 26.84 & 154 & 51.00\end{array}$ $1319928.061920 .72 \quad 15513.06$

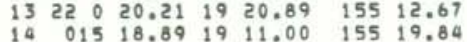

$\begin{array}{lllllll}14 & 016 & 55.67 & 19 & 10.97 \quad 155 & 20.26\end{array}$

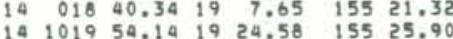
$\begin{array}{llllllll}14 & 11 & 1 & 6.11 & 19 & 24.81 & 155 & 25.89 \\ 14 & 13 & 5 & 2.38 & 19 & 20.33 & 155 & 13.14\end{array}$

$\begin{array}{lllllll}14 & 2154 & 58.44 & 19 & 31.51 & 155 & 46.81\end{array}$ $\begin{array}{lllllll}15 & 341 & 24.57 & 19 & 20.70 & 155 & 10.98 \\ 15 & 537 & 14.04 & 19 & 20.74 & 155 & 11.12\end{array}$

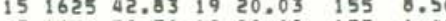
$15 \quad 1838 \quad 50.501920 .08 \quad 155 \quad 6.48$ $15201828.051918 .86 \quad 155 \quad 13.51$ $16 \quad 8 \quad 456.101921 .43 \quad 15457.23$ $16 \quad 1338 \quad 9.91 \quad 1919.07 \quad 15511.49$

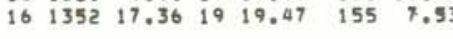
$\begin{array}{lllllll}16 & 1427 & 53.32 & 19 & 20.36 & 155 & 0.65\end{array}$ $\begin{array}{lllllll}16 & 1603 & 46.56 & 19 & 20.88 & 155 & 6.65\end{array}$

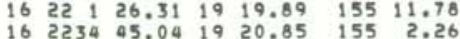
$17174130.031925 .62 \quad 15528.74$ $\begin{array}{rrrrrrrr}18 & 0 & 9 & 43.35 & 19 & 23.02 & 155 & 2.18 \\ 18 & 4 & 1 & .17 & 19 & 24.75 & 154 & 53.52\end{array}$ $1816859.85 \quad 1923.74 \quad 155 \quad 5.58$
PEPTH AMP OUR NR GAP RMS MIN ERH ERZ NO
KM MAF UAG NR NS DEG SEC OIS KM KM FM REMK $\begin{array}{lllllllllll}5.90 & 1 . h & 2.0 & 27 & 0 & 91 & .12 & 5 & .6 & 1.0 & 27 \\ 0 & 5 E R\end{array}$

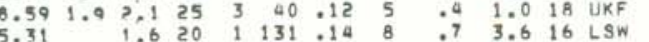

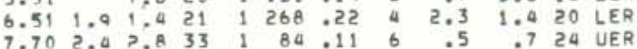
$\begin{array}{lllllllllllllll}8.52 & 2.4 & 2.7 & 32 & 2 & 139 & .11 & 4 & .5 & .7 & 27 & \text { POL }\end{array}$

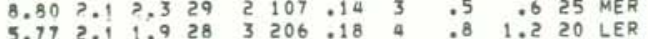

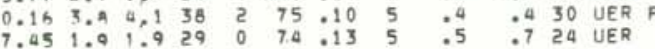

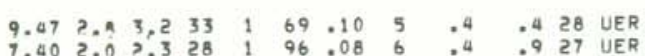

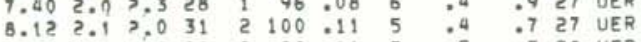

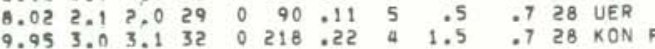
$\begin{array}{llllllllllll}7.98 & 1.9 & 2.2 & 32 & 1 & 94 & .13 & 5 & .5 & .8 & 29 & \text { UER }\end{array}$

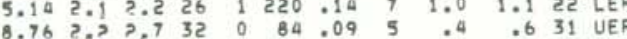

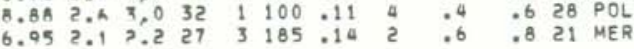
\begin{tabular}{lllllllllll}
8.36 & 2.1 & 2.5 & 31 & 4 & 272 & .19 & 6 & 1.3 & .8 & 27 \\
\hline
\end{tabular}

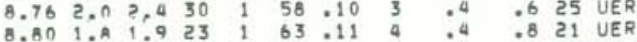

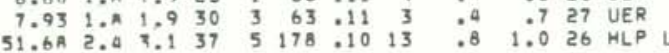
$99.85 \quad 2.220 .1 \quad 32 \quad 5 \quad 177 \quad .13 \quad 13 \quad .9 \quad 1.120$ HLP L

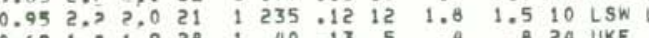
\begin{tabular}{lllllllllll}
0.69 & $1 . A$ & 1.9 & 28 & 1 & 40 & .13 & 5 & .4 & .8 & 24 \\
\hline
\end{tabular}

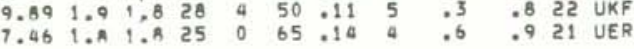
$\begin{array}{llllllllllll}9.76 & 1.6 & 2.3 & 25 & 5 & 87 & .13 & 2 & .5 & .5 & 24 & K O N\end{array}$

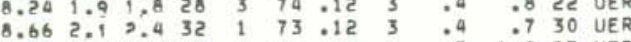

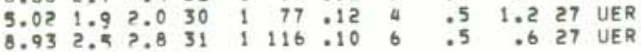
$\begin{array}{llllllllllll}0.34 & 2.1 & 2,5 & 32 & 0 & 73.14 & 3 & .5 & .6 & 26 & \text { POL }\end{array}$

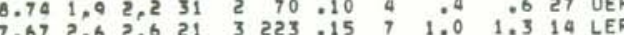

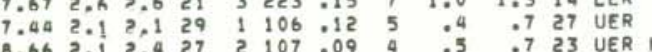

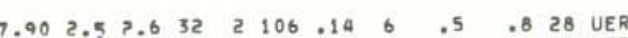

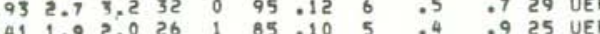

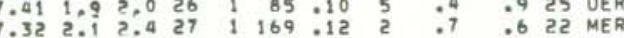

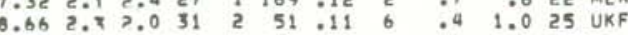

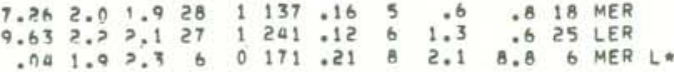


ORIGIN TIME LAT N LON W OEPTH AMP OIIR GAP RMS MIN ERH ERZ NO

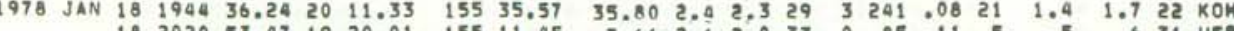

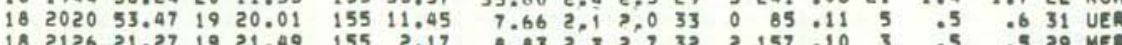

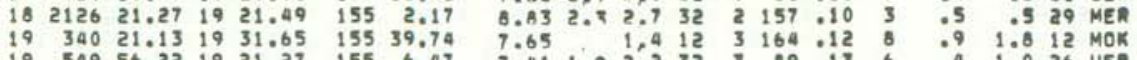
$\begin{array}{lllllllllllllll}19 & 623 & 1.78 & 19 & 1.87 & 155 & 19.71 & 38.17 & 2.3 & 0.0 & 32 & 2 & 220 & .05 & 20\end{array}$

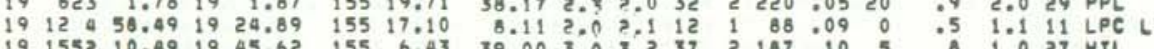

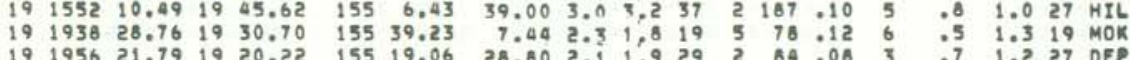

$\begin{array}{lllllllllllllllllll}20 & 340 & 15.74 & 19 & 20.30 & 155 & 4.05 & 7.40 & 2.1 & 1.9 & 31 & 1 & 122 & .12 & 2 & .7 & .7 & 29 & \text { MER }\end{array}$

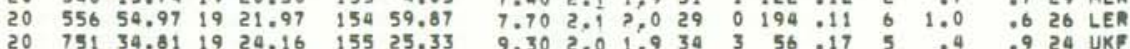

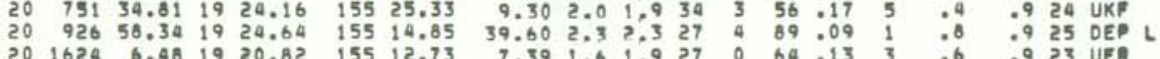

$20 \quad 2032 \quad 25.42 \quad 1924.60 \quad 154 \quad 48.22$

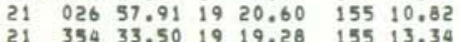
$21 \quad 548 \quad 39.62$ 19 $19.80 \quad 155510.44$ $21 \quad 12226.211921,37 \quad 155 \quad 2,91$ $\begin{array}{llllllll}21 & 15 & 9 & 18.06 & 19 & 22.60 & 155 & 28.18\end{array}$

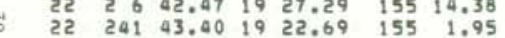
$\begin{array}{lllllll}22 & 423 & 24.93 & 19 & 20.74 & 155 & 2.04 \\ 22 & 541 & 32.82 & 19 & 18.84 & 155 & 7.13\end{array}$

$\begin{array}{lllllll}22 & 1349 & 30.34 & 19 & 32.82 & 155 & 48.47\end{array}$ $22 \quad 1522 \quad 32.111927 .84 \quad 155 \quad 51.42$

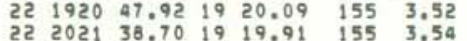

$\begin{array}{lllllll}22 & 2026 \quad 15.80 \quad 19 & 20.10 & 155 & 3.42\end{array}$ (1) $30.65 \quad 155 \quad 3.87$ $22 \quad 204439.79 \quad 1920.68 \quad 155 \quad 3.68$

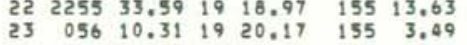

$\begin{array}{llllllll}23 & 128 & 8.88 & 19 & 17.61 & 155 & 27.25\end{array}$ $23528 \quad 10.651919 .16 \quad 155 \quad 16.38$ $\begin{array}{rrrrrrr}23 & 817 & 35.54 & 19 & 16.93 & 155 & 6.44 \\ 23 & 1034 & 21.12 & 19 & 19.05 & 155 & 52.35\end{array}$

$\begin{array}{llllllll}23 & 21 & 9 & 41.73 & 19 & 30.90 & 155 & 27.62\end{array}$ $24 \quad 332 \quad 36.771921 .24 \quad 155 \quad .78$

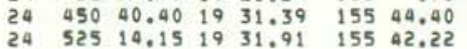

$\begin{array}{lllllll}24 & 557 \quad 1.66 \quad 19 & 25.26 & 155 & 26.07\end{array}$ $\begin{array}{lllllll}24 & 849 & 1.92 & 19 & 19.08 & 155 & 6.62 \\ 24 & 1518 & 17.04 & 19 & 20.44 & 155 & 6.41\end{array}$

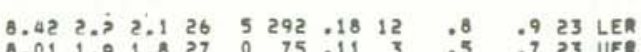
$\begin{array}{llllllllllll}0.03 & 2.4 & 2.5 & 33 & 0 & 61 & .12 & 1 & .4 & .7 & 29 & \text { UKF }\end{array}$

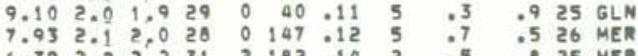

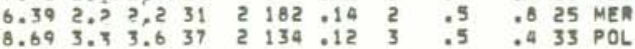
$\begin{array}{lllllllllllll}9.76 & 2,2 & 15 & 2 & 108 & .13 & 6 & .7 & .9 & 14 & \text { KON }\end{array}$

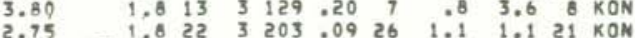

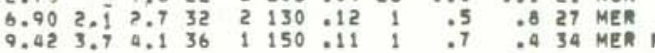
$\begin{array}{llllllllllllllll}7.36 & 2 . j & 0.4 & 32 & 2 & 126 & .13 & 1 & .6 & .7 & 29 & \text { MER }\end{array}$

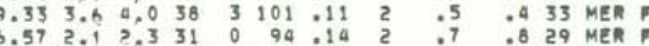

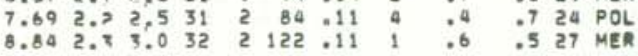

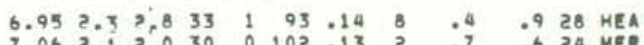

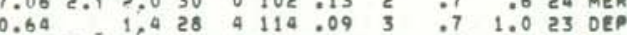

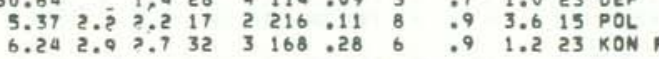

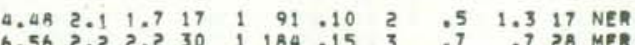

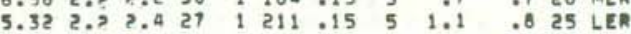

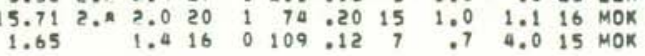

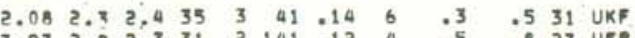

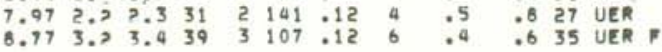

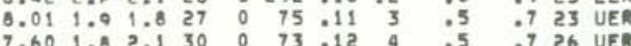

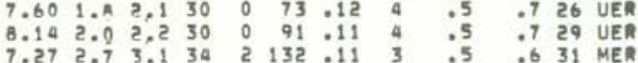

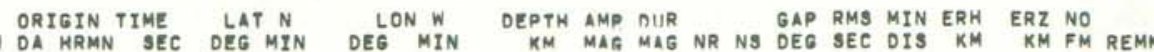

1078 JAN 24105232.031925 .5115527 .96 $25 \quad 3831.541920 .81 \quad 155 \quad 7.16$ $25516 \quad 41.36$ 19 $23.29 \quad 15517.16$ $25 \quad 55917.75 \quad 19 \quad 19.46 \quad 155 \quad 13.10$ 2573011.721819 .0515517 .08

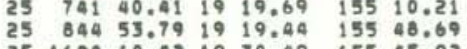
$25 \quad 1628 \quad 10,82 \quad 19 \quad 30.49 \quad 155 \quad 45.92$

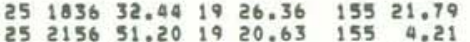

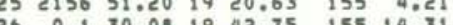
26 124 $43.261926 .86 \quad 15521.37$ $\begin{array}{llllllll}26 & 10 & 3 & 3.39 & 19 & 24.03 & 155 & 17.17\end{array}$

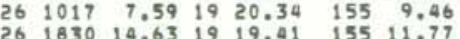

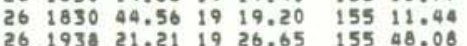
$\begin{array}{lllllll}27 & 10 & 5 & 34.95 \quad 19 & 17.20 \quad 155 & 21.35\end{array}$ $27 \quad 1629 \quad 36.02 \quad 1920.29 \quad 155 \quad 2.81$

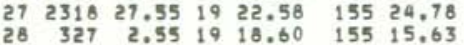

$\begin{array}{lllllll}28 & 7 & 4 & 6.99 & 19 & 56.92 \quad 155 \quad 20.24\end{array}$ $28183420.78 \quad 1919.77 \quad 155 \quad 10.24$ $\begin{array}{lllllll}28 & 1952 & 22.03 & 19 & 20.69 & 155 & 11.66 \\ 28 & 2212 & 25.72 & 19 & 27.82 & 154 & 52.75\end{array}$ $\begin{array}{lllllll}28 & 2230 & 54.37 & 19 & 22.73 & 155 & 4.42\end{array}$ $\begin{array}{llllll}818 & 56.79 & 19 & 21.51 & 155 \quad .46\end{array}$ $\begin{array}{rrrrrrr}30 & 038 & 2.57 & 19 & 22.00 & 156 & 22.19 \\ 30 & 18 & 41.34 & 19 & 17.61 & 155 & 21.83\end{array}$ $\begin{array}{lllllll}30 & 451 & 8.22 & 19 & 31.17 & 155 & 39.92\end{array}$ $\begin{array}{lllllll}30 & 918 & 51.54 & 19 & 20.34 & 155 & 4.22\end{array}$ 30124136.941921 .25 155 6.05

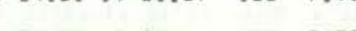
$\begin{array}{lllllll}30 & 18 & 88.42 & 19 & 20.68 & 155 & 2.39\end{array}$ $\begin{array}{lllllll}31 & 539 & 15.03 & 19 & 23.92 & 155 & 15.03\end{array}$ $\begin{array}{lllllll}31 & 1030 & 42.58 & 19 & 20.42 & 155 & 2.50 \\ 31 & 1637 & 59.47 & 19 & 19.15 & 155 & 6.13\end{array}$ $\begin{array}{lllllll}31 & 2219 & 45.73 \quad 19 & 26.94 & 155 & 27.49\end{array}$

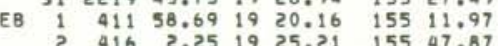

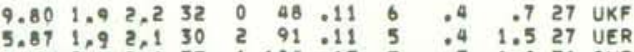

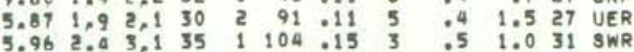

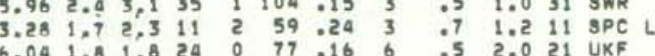

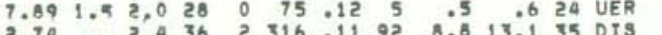

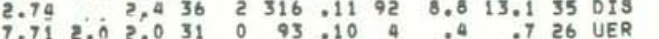

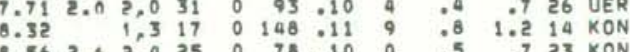
$\begin{array}{rrrrrrrrrrrr}8.92 & 1.9 & 1,7 & 23 & 2 & 44 & 014 & 6 & .4 & 1.0 & 21 & \text { UKF } \\ 6.12 & 2.1 & 2,1 & 29 & 0 & 108 & 0.13 & 3 & .7 & 1.0 & 25 & \text { MER }\end{array}$

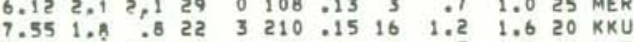

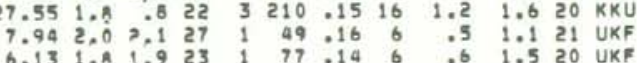
$\begin{array}{llllllllll}1.17 & 1 & 53 & .12 & 2 & .3 & .5 & 13 & \mathrm{SPC}\end{array}$

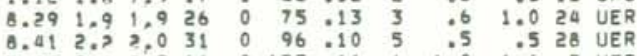

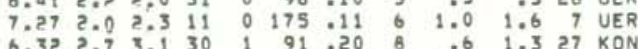

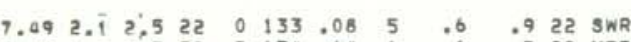

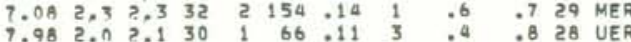
$\begin{array}{rrrrrrrrrrrr}9.94 & 2.1 & 1.8 & 33 & 1 & 56 & 12 & 5 & .5 & .7 & 29 & \text { UKF } \\ 7.14 & 1.9 & 2.0 & 27 & 0 & 131 & .09 & 4 & .5 & .8 & 24 & \text { KOA }\end{array}$ $\begin{array}{lllllllllllll}9.92 & 2.6 & 2.8 & 28 & 2 & 267 & .09 & 23 & 1.1 & .6 & 24 & k K U\end{array}$

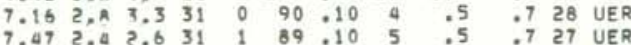

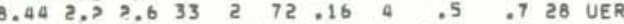
$6.892 .72 .326 \quad 0261.17$ 2 2.3 .8 26 LER $\begin{array}{llllllllllll}7.62 & 2.0 & 2.0 & 28 & 0 & 87 & .10 & 3 & .5 & .8 & 26 & \text { MER }\end{array}$

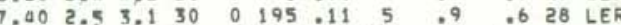

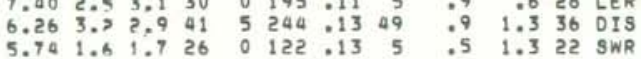

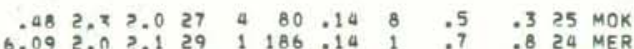
$\begin{array}{lllllllllll}0.37 & 2.4 & 2.7 & 33 & 2 & 121 & .11 & 2 & .5 & .6 & 31 \\ 0.9 E R\end{array}$

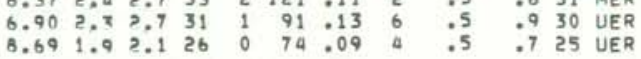
$\begin{array}{llllllllllll}6.91 & 2.1 & 2.2 & 29 & 1 & 170 & .13 & 2 & .6 & .6 & 24 & \text { MER }\end{array}$

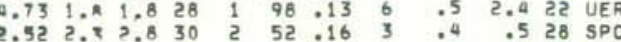
$\begin{array}{llllllllllll}6.17 & 2.4 & 2.6 & 30 & 3 & 175 & .17 & 1 & .7 & .8 & 57 & \text { MER }\end{array}$

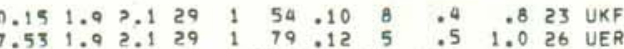

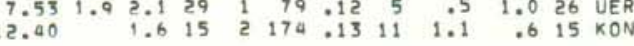


ORIGIN TIME LATN LON W OEPTH AMP OUR
DA HRMN SEC OEG MIN DEG MIN RMS MIN ERH ERZ NO
KM MAG MAG NR NS DEG SEC OIS KM KM FM REMK

1978 FEB $2 \quad 7 \quad 5 \quad 28.78 \quad 1920.68 \quad 155 \quad 3.65$ $\begin{array}{lllllll}1014 & 6.84 & 19 & 18.57 \quad 155 & 23.65\end{array}$

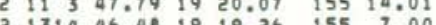

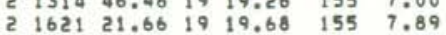

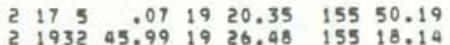
$2327 \quad 53.001920 .26 \quad 15511.91$

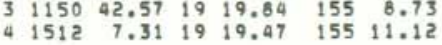
$\begin{array}{lllllll}4 & 1826 & 39.67 & 19 & 18.94 & 155 & 15.55 \\ 4 & 2053 & 26.80 & 20 & 2.94 & 155 & 44.01\end{array}$

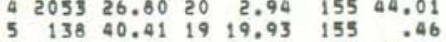
$\begin{array}{lllllll}5 & 138 & 40.41 & 19 & 19.93 & 155 & .46 \\ 5 & 144 & 4.17 & 19 & 20.87 & 155 & 12.98\end{array}$ $5 \quad 1629 \quad 54.07 \quad 19 \quad 20.06 \quad 155 \quad 7.92$ $\begin{array}{lllllll}5 & 1829 & 54.07 & 19 & 20.06 & 155 & 7.92 \\ 5 & 1845 & 25.94 & 19 & 24.80 & 155 & 28.95\end{array}$ $2034 \quad 42.791918 .26 \quad 15516.53$

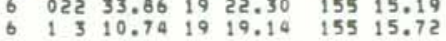
$\begin{array}{lllllll}6 & 128 & 20.17 & 19 & 19.35 & 155 & 15.43\end{array}$

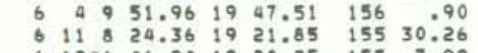
$\begin{array}{lllllll}6 & 1241 & 46.94 & 19 & 20.25 & 155 & 7.99 \\ 6 & 1525 & 11.99 & 19 & 19.85 & 155 & 11.70\end{array}$ $\begin{array}{llllllll}6 & 2159 & 19.36 & 19 & 23.37 & 155 & 30.01\end{array}$ $2257 \quad 45.281923 .59 \quad 155$
06.75 \begin{tabular}{l}
$01525.781921 .09 \quad 155 \quad 3.60$ \\
\hline
\end{tabular}

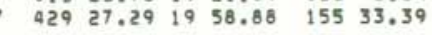

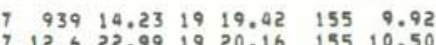
$123826801921.69 \quad 1552.19$

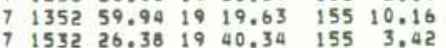
$\begin{array}{lllllll}7 & 1717 \quad 1.32 \quad 19 & 10.55 \quad 155 & 22.23\end{array}$

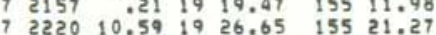
$\begin{array}{lllllll}8 & 328 & 42.46 & 19 & 20.65 & 155 & 8.07 \\ 8 & 553 & 12.50 & 19 & 33.62 & 154 & 52.47\end{array}$ $\begin{array}{llllllll}8 & 6 & 6 & 10.51 & 19 & 10.68 & 155 & 13.58\end{array}$ $8 \quad 1851 \quad 54.52 \quad 19 \quad 18.31 \quad 155 \quad 21.25$ 9 5 $559.56 \quad 1923.15 \quad 155 \quad 16.09$

$9 \quad 643 \quad 1.521918 .23 \quad 155 \quad 13.53$ $10 \quad 347 \quad 49.64$ 19 $19.92 \quad 155 \quad 13.22$ $10 \quad 625 \quad 58.171920 .21 \quad 15512.50$

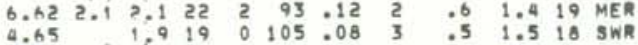

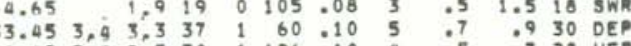

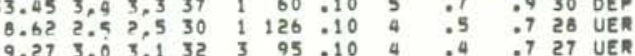
$\begin{array}{rrrrrrrrrrrr}5.78 & 2.6 & 1.9 & 22 & 0 & 176 & .12 & 9 & .9 & 1.3 & 18 & \text { KON } \\ 15.45 & 3.5 & 7.5 & 37 & 1 & 58 & .11 & 2 & .9 & .3 & 30 & \text { INT F }\end{array}$ $9.392 .32 .228 \quad 2 \quad 78.1150 .5$.7 26 UER

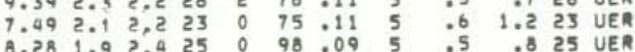
$\begin{array}{llllllllllllll}7.88 & 1.0 & 1.6 & 20 & 0 & 119 & .08 & 4 & .5 & .9 & 13 & K O A\end{array}$ $\begin{array}{llllllllllll}1.70 & 1.8 & 2.0 & 24 & 5 & 153 & -12 & 10 & 1.1 & 1.3 & 19 & \mathrm{KOH}\end{array}$

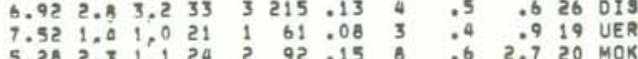

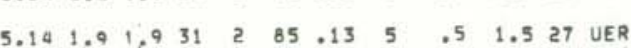
$\begin{array}{rlllllllllll}5.14 & 1.9 & .9 & 31 & 2 & 85 & .13 & 5 & .5 & 1.5 & 27 & \text { UER } \\ 8.04 & 1.8 & 1.9 & 28 & 0 & 67 & .11 & 5 & .6 & 1.3 & 23 & \text { UKF }\end{array}$

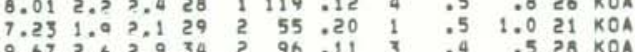
$\begin{array}{llllllllllllll}9.34 & 2.0 & 2.7 & 33 & 2 & 88 & .15 & 4 & .5 & .730 & 30 A\end{array}$

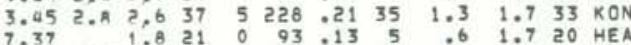
$\begin{array}{llllllllllllll}8.98 & 2.7 & 3.1 & 31 & 0 & 86 & .10 & 5 & .5 & .6 & 30 & \text { UER } \\ 7.27 & 1.7 & 1.6 & 21 & 0 & 86 & .10 & 5 & .6 & 1.2 & 20 & \text { UER }\end{array}$ $\begin{array}{rrrrrrrr}9.22 & 2.3 & 2.3 & 33 & 0 & 55 & .11 & 5 \\ 3 & .4 & .930 & 30 K & \text { MOK }\end{array}$

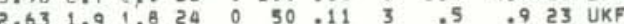

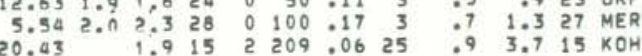
$\begin{array}{lllllllllll}8.33 & 2.03 .0 & 33 & 0 & 97 & .09 & 5 & .5 & .6 & 29 & \text { UER }\end{array}$

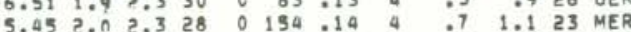

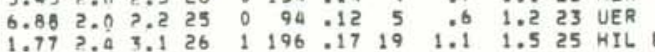
$\begin{array}{lllllllllllll}5.73 \quad 2.2 & 23 & 2 & 185 & .11 & 15 & 1.3 & 1.2 & 19 & \text { LSW L }\end{array}$

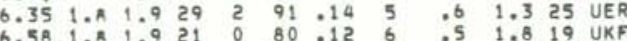
$\begin{array}{lll:lllllllll}5.97 & 1.9 & 8 & 25 & 0 & 118 & : 12 & 4 & .6 & 1.4 & 25 & \text { UER } \\ 3.87 & 2.1 & 1.9 & 26 & 5 & 242 & .34 & 9 & 2.3 & 4.9 & 23 & \text { HIL }\end{array}$ $\begin{array}{llllllllllll}7.77 & 2.0 & 2.5 & 28 & 0 & 73 & -13 & 3 & .6 & .8 & 27 & P O L\end{array}$

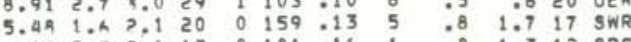

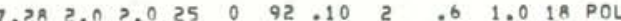

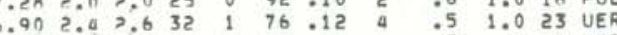

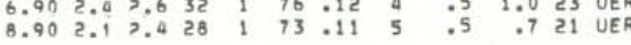

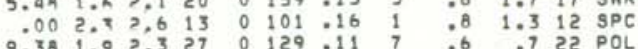
ORIGIN TIME LAR MON DA HRMN SEC OEG MIN DEG MON W OEPTH AMP RUR N GAP RMS MIN ERH ERZ NO
KM MAR MAG NR NS DEG SEC OIS KM KM FM REMK

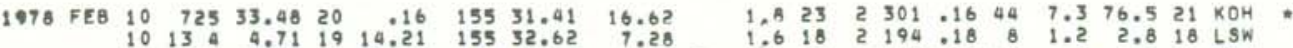

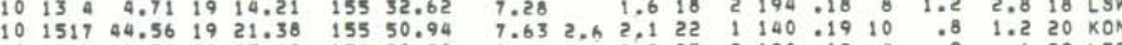

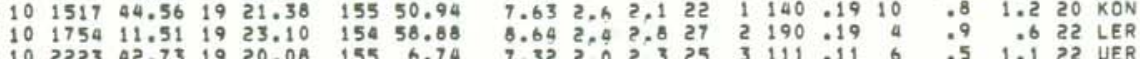
$\begin{array}{llllllllllllllllllll}10 & 23 & 3 & 39.24 & 19 & 21.10 & 155 & 24.57 & 8.97 & 2 . ? & 2.3 & 37 & 2 & 71 & .14 & 3 & .4 & .6 & 28 & \text { SWR }\end{array}$

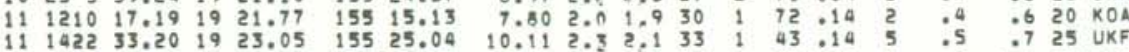

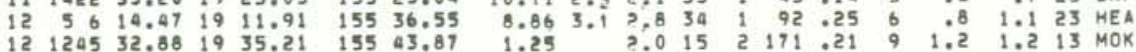
$\begin{array}{llllllllllllllllllll}12 & 14 & 3 & 16.19 & 19 & 8.14 & 155 & 27.07 & 49.11 & 2.6 & 2.9 & 30 & 0 & 171 & .07 & 22 & 1.0 & 2.5 & 26 & \mathrm{LSW} L\end{array}$

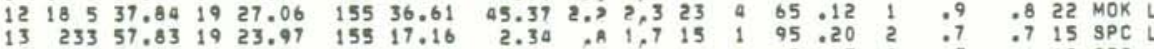

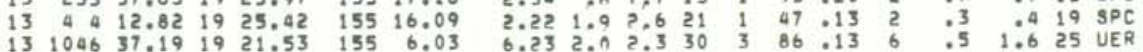

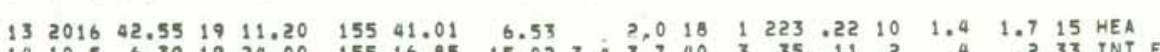

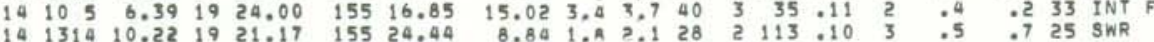

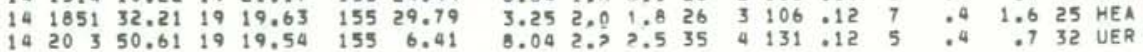
$\begin{array}{lllllllllllllllllll}14 & 2335 & 47.80 & 19 & 26.12 & 155 & 15.92 & 21.75 & 2.1 & 2.3 & 38 & 5 & 39 & .12 & 3 & .5 & .7 & 28 & \text { INT }\end{array}$

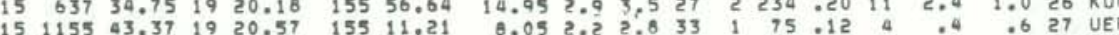

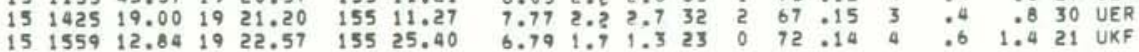

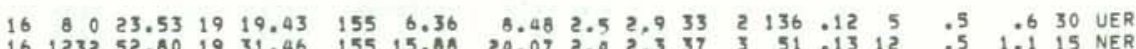

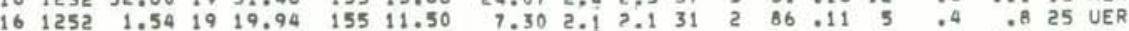

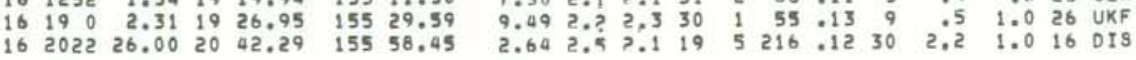
$\begin{array}{llllll}16 & 2034 & 25.39 & 19 & 20.37 \quad 155 & 12.02\end{array}$ $0.191920 .80 \quad 155 \quad 6.09$ $17 \quad 2120.16 \quad 1919.92 \quad 155 \quad 7.68$

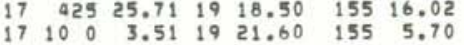
$\begin{array}{lll}17 & 1334 & 21.24 \quad 1923.64 \quad 155 \quad 24.35\end{array}$ 172059

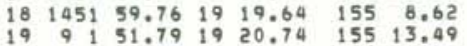
$\begin{array}{llllllllllllllllllll}19 & 1149 & 47.68 & 19 & .63 & 155 & 25.72 & 41.20 & 3.7 & 3.1 & 37 & 1 & 215 & .06 & 17 & 1.0 & 2.0 & 35 & \text { LSW } & \end{array}$

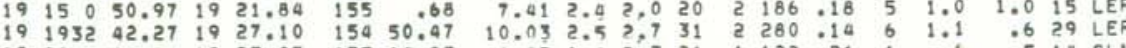

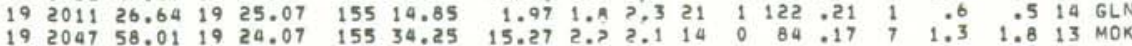

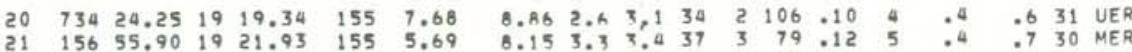

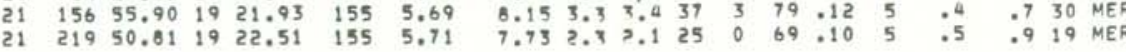




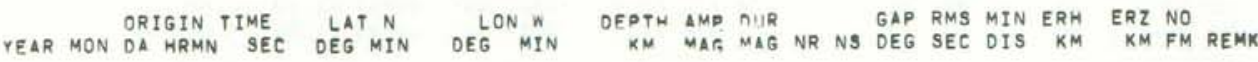

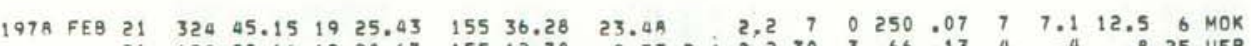

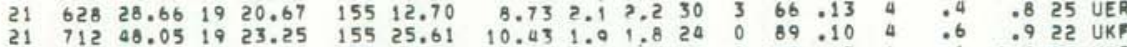

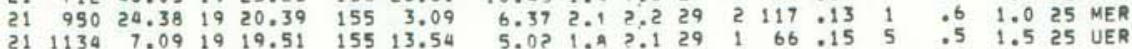

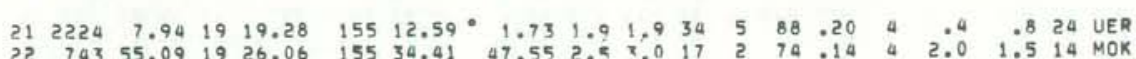

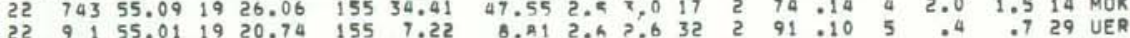

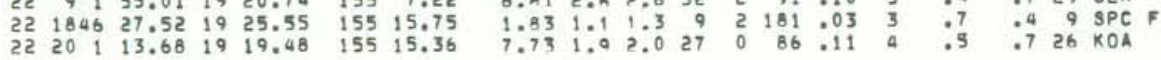
$22 \quad 2056 \quad 15.44 \quad 1929.64 \quad 15515.91 \quad 4.741 .4 \quad 1.1 \quad 6 \quad 1326.17 \quad 9 \quad 3.831 .9 \quad 6$ GLN *

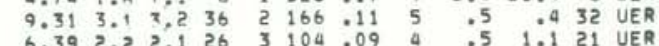

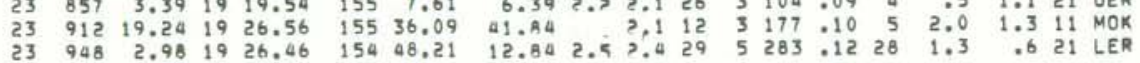
$\begin{array}{llllllllllllllllll}23 & 1337 & 41.10 & 19 & 25.40 & 155 & 16.73 & 5.97 & 2.0 & 2.3 & 13 & 1 & 140 & .11 & 2 & .6 & .6 & 12 \\ \text { LPC }\end{array}$

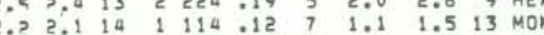

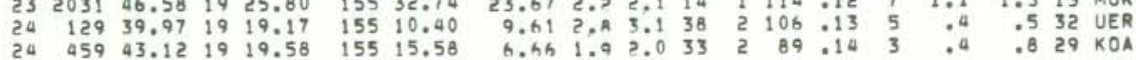

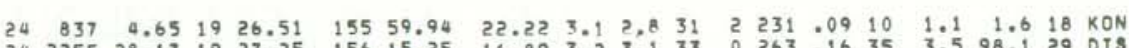

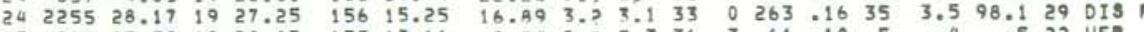

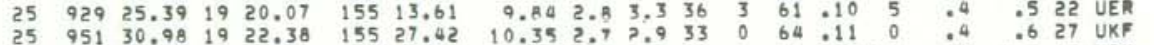

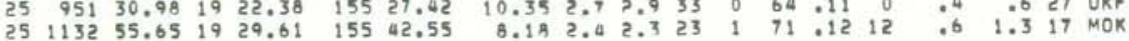

$\begin{array}{lllllllllllllllllll}25 & 1146 & 4.59 & 19 & 26.23 & 155 & 18.11 & 14.49 & 2.1 & 2.4 & 37 & 2 & 58 & .09 & 2 & .4 & .3 & 31 & \text { INT }\end{array}$

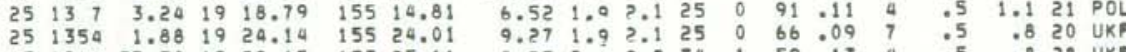

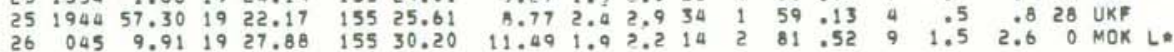

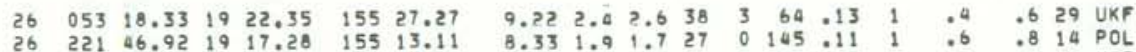

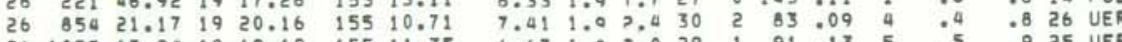

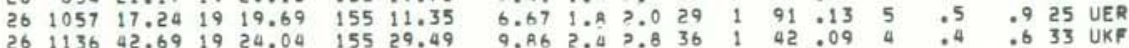
$\begin{array}{lllllllllllllllllll}26 & 1213 & 05.55 & 19 & 11.79 & 155 & 38.38 & 8.65 & 2 . ? & 2.1 & 30 & 2 & 101 & .23 & 6 & .8 & 1.2 & 24 & \text { HEA } \\ 26 & 1255 & 50.18 & 19 & 18.46 & 155 & 14.51 & 6.98 & 1.9 & 1.7 & 24 & 0 & 116 & .12 & 3 & .6 & .9 & 21 & \text { POL }\end{array}$

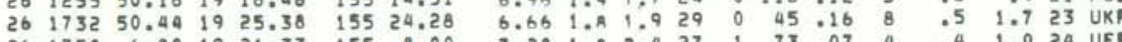

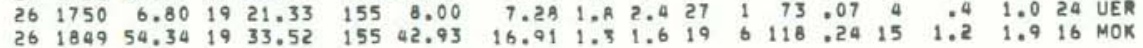

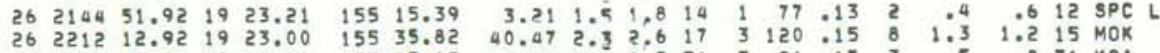

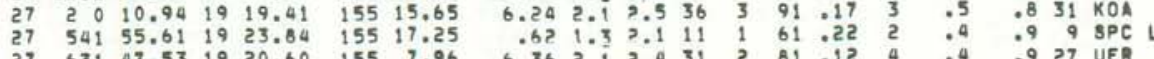

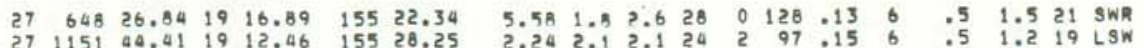

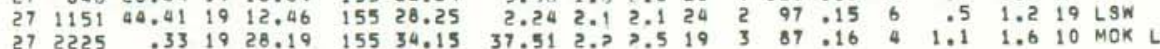

YEAR MON ORIGIN TIME 1978 FEB $28 \quad 0.40 \quad 49.81 \quad 1920.73 \quad 155 \quad 13.28$ 2822433.02 19 18,80 155 15.63

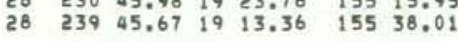

$28 \quad 255 \quad 23.32 \quad 1926.55 \quad 15524.10$ $28 \quad 1928 \quad 51.35$ 19 $20.86 \quad 155 \quad 24.34$

MAR $\quad \begin{array}{lrrrrrr}256 & 43.06 & 19 & 18.90 & 155 & 13.58 \\ 1 & 1438 & 49.92 & 19 & 25.31 & 154 & 55.01\end{array}$

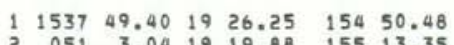
74513.7619 2 $1225 \quad 35.66 \quad 1925.13 \quad 15455.88$ $\begin{array}{lllllll}2 & 1843 & 8.60 & 19 & 23.58 & 155 & 2.26 \\ 2 & 1920 & 15.35 & 19 & 19.28 & 155 & 6.23\end{array}$ $22018 \quad 51.691958 .03 \quad 15533.38$

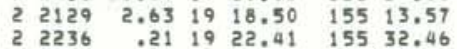

$\begin{array}{lllllll}3 & 1451 & 51.39 & 19 & 21.57 \quad 155 & 15.02\end{array}$

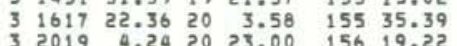

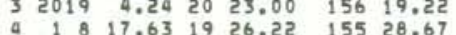
a $188 \quad 17.63 \quad 1926.22 \quad 15528.67$

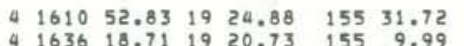

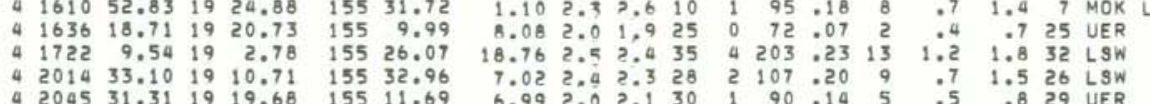

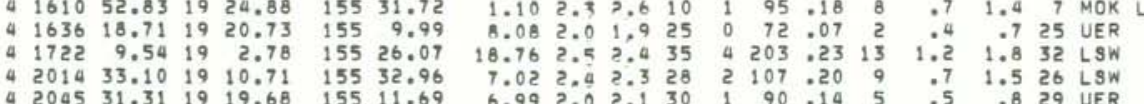

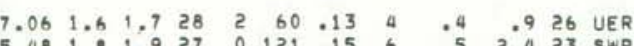

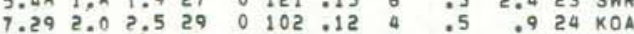
$\begin{array}{llllllllllll}2.26 & 1 . ? & 1.6 & 16 & 3 & 100 & .14 & 3 & .3 & .5 & 12 & \text { SPC } \\ 7.44 & 2.9 & 2.3 & 31 & 1 & 94 & .22 & 3 & .8 & 1.2 & 24 & \text { HEA }\end{array}$ $\begin{array}{lllllllllllll}7.71 & 1.7 & 1.9 & 28 & 1 & 54 & 17 & 6 & .5 & 1.7 & 26 & \text { UKF }\end{array}$ 10.792 .32 .1257 0 $49.09 \quad 4 \quad$ :5 1.025 UKF .41 .122 POL

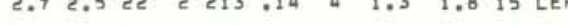
$\begin{array}{rrrrrrrrrrrr}8.54 & 2.6 & 2.3 & 21 & 1 & 275 & .14 & 7 & 1.5 & .9 & 17 & \text { LER } \\ 9.72 & 3.7 & 4.0 & 30 & 3 & 67 & .10 & 5 & .4 & .6 & 22 & \text { UER F }\end{array}$ 7.412 .72 .231 \% 82,125 . 5 .5 926 UER

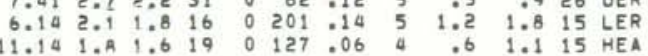
$\begin{array}{lllllllllllll}8.97 & 2.1 & 1.9 & 21 & 0 & 125 & -11 & 4 & .6 & 1.0 & 21 & \text { MER }\end{array}$

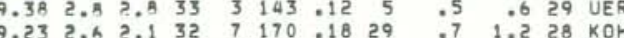

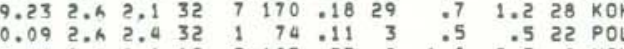

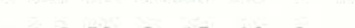
$\begin{array}{rlllllllllll}5.35 & 0.5 & ? .9 & 30 & 2 & 63 & .10 & 2 & .4 & .5 & 27 & \mathrm{KOA}\end{array}$

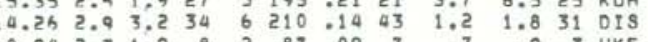

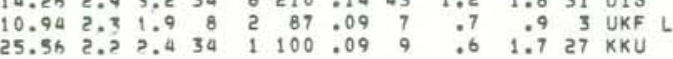

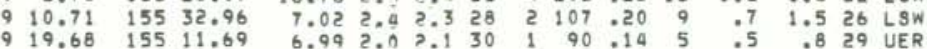
$\begin{array}{llllllllllllllllllll}5 & 028 & 48.93 & 19 & 20.36 & 155 & 13.37 & 8.02 & 2.0 & 2.2 & 25 & 3 & 63 & .12 & 4 & .4 & .9 & 20 & \text { UER } \\ 5 & 218 & 56.05 & 19 & 24.81 & 155 & 34.35 & 27.34 & 2.7 & 2.4 & 9 & 1 & 92 & .08 & 6 & 1.6 & 5.1 & 8 & \text { MOK L }\end{array}$

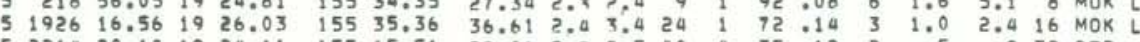

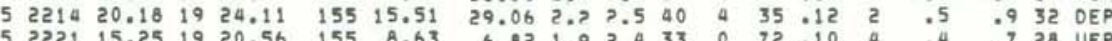

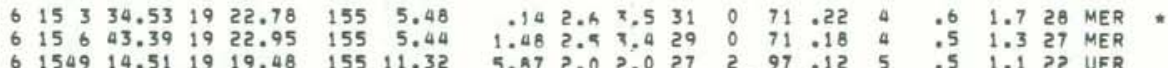

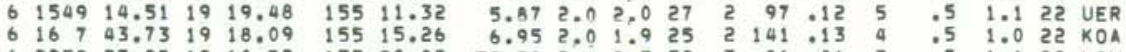

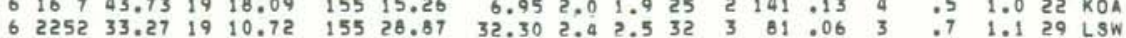

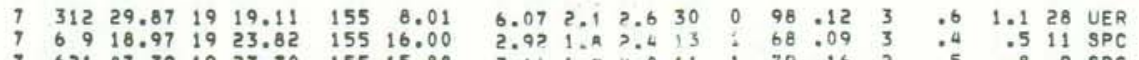

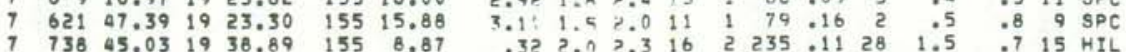

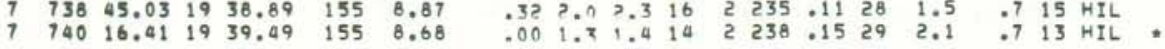

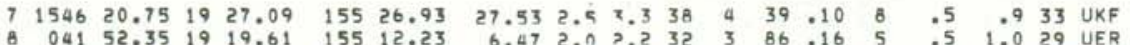

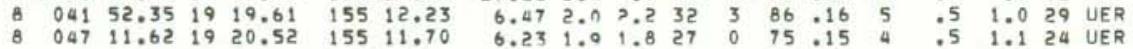




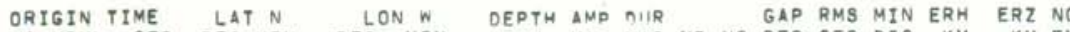
YEAR MON OA HRMN SEC DEG MIN DEG MIN KM MAG MAG NR NS DEG SEC DIS KM KM FM REMK

197 B MAR

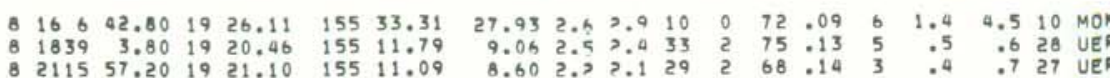

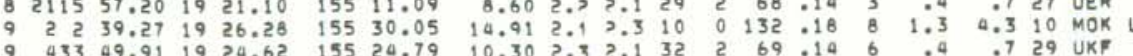

$9752 \quad 36.12 \quad 19 \quad 18.86 \quad 15513.62$ Q $115919.901917 .51 \quad 15513.64$

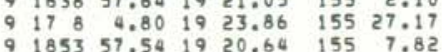

$\begin{array}{lllllll}9 & 2316 & 16.99 & 19 & 20.21 & 155 & 8.57\end{array}$

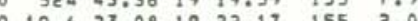
$102017 \quad 49.50 \quad 1925.74 \quad 155 \quad 15.6330$ $10 \quad 215551.22 \quad 1926.14 \quad 155 \quad 36.43$ $11 \quad 014 \quad 22.00 \quad 1922.75 \quad 155 \quad .44$

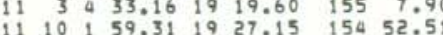
$\begin{array}{rrrrrrr}11 & 1529 & 24.84 & 19 & 19.04 & 155 & 13.52 \\ 12 & 046 & 47.09 & 19 & 19.29 & 155 & 12.06\end{array}$ $\begin{array}{llllllll}12 & 12 & 0 & 34.04 & 19 & 15.99 & 155 & 32.22\end{array}$

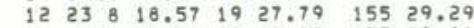
$\begin{array}{llllllll}13 & 846 & 24.08 & 19 & 19.88 & 155 & 12.06 \\ 13 & 936 & 13.84 & 19 & 31.89 & 155 & 27.70\end{array}$ $\begin{array}{llllllll}13 & 1115 & 27.10 & 19 & 20.58 & 155 & 10.35\end{array}$

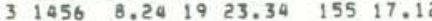
$13 \quad 183025.33 \quad 19 \quad 10.16 \quad 155 \quad 29.07$ $13 \quad 1947 \quad 46.87 \quad 1923.15 \quad 155 \quad 16.12$ $\begin{array}{lllllll}14 & 337 & 19.16 & 19 & 18.00 & 155 & 12.99\end{array}$

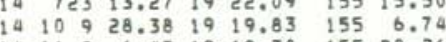
$\begin{array}{rrrrrrr}14 & 11 & 1.47 & 19 & 19.30 & 155 & 20.26 \\ 14 & 1129 & 51.15 & 19 & 19.88 & 155 & 6.78\end{array}$ $\begin{array}{lllllll}14 & 1512 & 24.54 & 19 & 22.18 & 155 & 4.52\end{array}$ 14 19 $46.18 \quad 1921.77 \quad 155 \quad 5.62$ $\begin{array}{rrrrrrr}14 & 2222 & 6.97 & 19 & 21.94 & 155 & .33 \\ 15 & 155 & 45.50 & 19 & 18.32 & 155 & 21.20\end{array}$

$\begin{array}{lllllll}15 & 226 & 11.74 & 19 & 24.27 & 155 & 26.28\end{array}$ $\begin{array}{lllllll}15 & 42 & 14.61 & 19 & 32.55 & 155 & 42.67 \\ 15 & 430 & 1.16 & 19 & 24.00 & 155 & 25.20\end{array}$ $\begin{array}{lllllll}15 & 430 & 30.93 & 19 & 24.01 & 155 & 25.17\end{array}$ $\begin{array}{lllllll}15 & 1130 & 49.87 & 19 & 20.99 & 155 & 1.88\end{array}$ $\begin{array}{lllllll}16 & 1043 & 50.85 & 19 & 20.51 & 155 & 6.57 \\ 16 & 1351 & 58.40 & 19 & 20.67 & 155 & 7.01\end{array}$

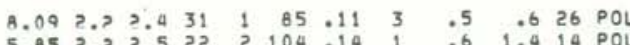

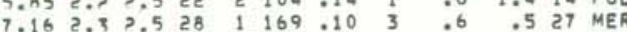
$\begin{array}{llllllllllll}9.06 & 2.7 & 2.1 & 34 & 1 & 64 & .13 & 3 & .4 & .8 & 32 & \text { UKF } \\ 9.05 & 2.5 & 2.6 & 31 & 1 & 85 & .12 & 4 & .4 & .7 & 29 & \text { UER }\end{array}$ $\begin{array}{llllllllllll}8.17 & 2.7 & 2.6 & 31 & 0 & 75 & .09 & 4 & .5 & .7 & 27 & \text { UER } \\ 9.55 & 3.5 & 3.3 & 37 & 3 & 93 & .10 & 4 & .4 & .5 & 30 & \text { UER }\end{array}$

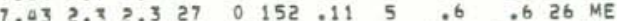

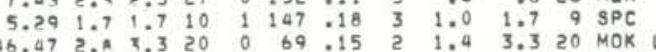
\begin{tabular}{llllllllllll}
7.11 & 2.1 & 0.2 & 25 & 0 & 173 & .19 & 5 & 1.0 & 1.1 & 24 \\
\hline & LER
\end{tabular}

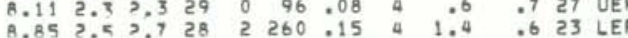

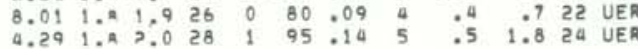
$\begin{array}{llllllllllllll}1.05 & 2.2 & 0.0 & 30 & 2 & 118 & .18 & 9 & .5 & .7 & 26 & \text { LSW }\end{array}$

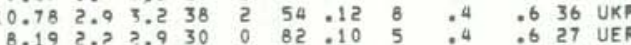

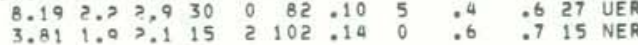
$\begin{array}{lllllllllllll}0.07 & 1.0 & 2.1 & 26 & 2 & 125 & .10 & 3 & .5 & .9 & 24 & \text { UER }\end{array}$

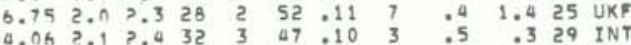

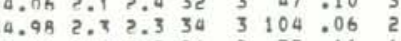
$0.19+1,20128$

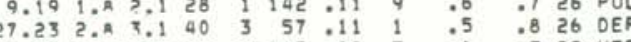

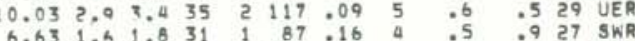

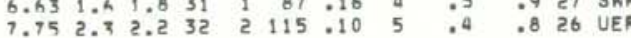
$\begin{array}{rrrrrrrrrrrr}8.56 & 2.7 & 2.5 & 31 & 1 & 85 & .09 & 4 & .5 & .5 & 30 & \text { MER } \\ 8.90 & 2.0 & 2.4 & 34 & 2 & 104 & .12 & 4 & .5 & .7 & 28 & \text { UER }\end{array}$ $\begin{array}{rrrrrrrrrrr}7.88 & 2.0 & 2.1 & 32 & 3 & 81 & .14 & 6 & .5 & .8 & 29 \\ 8.91 & 2.4 & 2.8 & 34 & 2 & 188 & .12 & 6 & .7 & .5 & 27 \\ 7.9 R\end{array}$

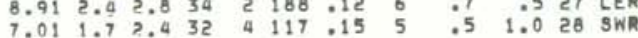
$\begin{array}{llllllllllll}7.59 & 2.2 & 2.3 & 36 & 3 & 62 & .14 & 4 & .4 & 1.2 & 35 & \text { UKF } \\ .12 & 1.5 & 3 & 26 & 6 & 83 & .13 & 7 & .4 & .4 & 24 & \text { MOK }\end{array}$

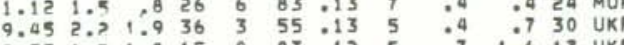
$\begin{array}{lllllllllllll}8.33 & 1.7 & 1.8 & 15 & 0 & 83 & .12 & 5 & .7 & 1.6 & 13 & \text { UKP } \\ 7.95 & 2.7 & ? .6 & 32 & 3 & 97 & .10 & 5 & .4 & .7 & 26 & \text { UER }\end{array}$ $\begin{array}{llllllllllll}4.33 & 2.1 & 0.2 & 26 & 2 & 178 & .11 & 3 & .6 & 1.2 & 20 & \text { MER } \\ 6.03 & 2.2 & 1.9 & 20 & 0 & 104 & 0.12 & 6 & .7 & 1.9 & 18 & \text { UER }\end{array}$

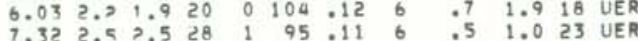

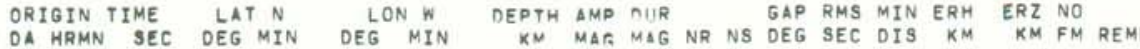
$\begin{array}{lllllll}16 & 146 & 9.41 & 19 & 59.71 & 155 & 20.10 \\ 16 & 1556 & 11.52 & 19 & 26.15 & 155 & 28.41\end{array}$ $16204338.021920 .86 \quad 1559.52$

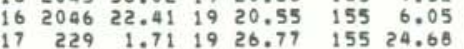
$17 \quad 246 \quad 14.09 \quad 19 \quad 20.14 \quad 155 \quad 13.08$ \begin{tabular}{l}
$7 \quad 1313 \quad 18.34 \quad 1920.45 \quad 155 \quad 11.55$ \\
\hline
\end{tabular}

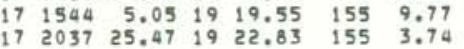
$17 \quad 2129 \quad 10.72 \quad 19 \quad 16.96 \quad 155 \quad 13.33$ $\begin{array}{lllllll}17 & 2129 & 10.72 & 19 & 18.96 & 155 & 13.33 \\ 18 & 345 & 44.97 & 19 & 22.70 & 155 & 3.21\end{array}$ $\begin{array}{llllllll}18 & 13 & 1 & 22.74 & 19 & 20.54 & 155 & 1.55\end{array}$ $\begin{array}{lllllll}18 & 1450 & 22.69 & 19 & 21.13 & 155 & 2.72 \\ 18 & 1622 & 30.02 & 19 & 11.77 & 155 & 9.26\end{array}$ $\begin{array}{lllllll}18 & 1711 & 4.88 & 19 & 20.94 & 155 & 1.49\end{array}$

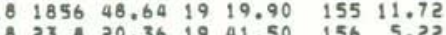

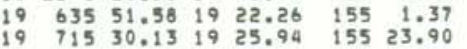
$\begin{array}{llllllll}19 & 8 & 2 & 18.33 & 19 & 22.18 & 155 & 6.27\end{array}$ 19213453.72 19 19.59 155 3.65 $191832 \quad 17.16 \quad 19 \quad 19.27 \quad 155 \quad 9.87$ $\begin{array}{lllllll}20 & 1148 & 11.40 & 19 & 2.28 & 155 & 27.44\end{array}$ $\begin{array}{lllllll}20 & 1856 & 0.79 & 19 & 19.16 & 155 & 16.25\end{array}$

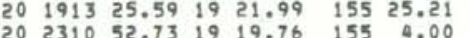
$\begin{array}{lllllll}21 & 354 & 14.25 & 19 & 18.96 & 155 & 6.87 \\ 21 & 7 & 14.95 & 19 & 20.47 & 155 & 11.82\end{array}$ $\begin{array}{lllll}152 & 17.87 & 1920.30 & 155 & 7.87\end{array}$ $22 \quad 726 \quad 9.581921 .86$ 155 17.96 $\begin{array}{lllllll}22 & 1318 & 35.80 & 19 & 21.75 & 155 & 7.35\end{array}$

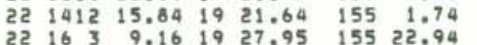

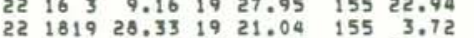

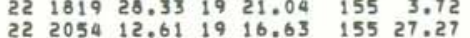
$\begin{array}{lllllll}23 & 1554 & 44.52 & 19 & 17.69 & 155 & 21.43 \\ 23 & 196 & 29.56 & 19 & 20.94 & 155 & 13.28\end{array}$

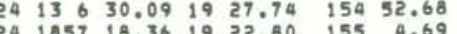
$25 \quad 2009.0219 \quad 19.46 \quad 155 \quad 10.78$ $\begin{array}{llrllll}25 & 626 & 2.29 & 19 & 19.89 & 155 & 8.17 \\ 25 & 657 & 56.43 & 19 & 20.88 & 155 & 10.74\end{array}$

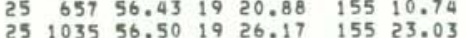

$\begin{array}{llllllllllll}8.012 .9 & 2.0 & 29 & 4 & 207 & .14 & 27 & .9 & .6 & 23 & \mathrm{KKU} F & \mathrm{~F}\end{array}$

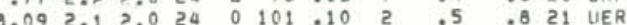

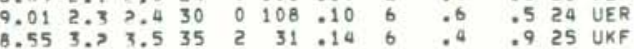
$\begin{array}{llllllllllll}9.75 & 3.0 & 3.2 & 37 & 2 & 68 & .10 & 5 & .4 & .5 & 26 & \text { UER }\end{array}$

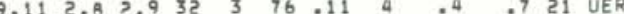

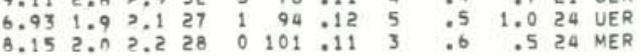
$\begin{array}{llllllllllll}7.65 & 2.0 & 2.0 & 31 & 0 & 77 & .13 & 4 & .5 & .7 & 26 & \text { POL }\end{array}$

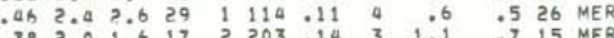

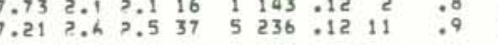
$\begin{array}{llllllllllll}7 . \text {. } & 2.1 & 1.8 & 23 & 3 & 191 & .10 & 3 & .5 & .8 & 15 & \text { MER }\end{array}$ $\begin{array}{rrrrrrrrrrrr}6.44 & 1.7 & .4 & 16 & 0 & 85 & -12 & 5 & .6 & 1.4 & 14 & \text { UER } \\ 15.94 & & 9 & 20 & 4 & 287 & 17 & 26 & 2.7 & 8.0 & 17 & \text { KON }\end{array}$

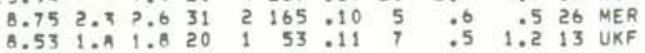
$\begin{array}{llllllllllllll}7.09 & 5.2 & 0.1 & 27 & 1 & 74 & .11 & 6 & .4 & 1.0 & 23 & \text { UER }\end{array}$

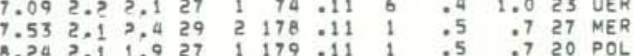

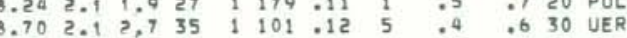

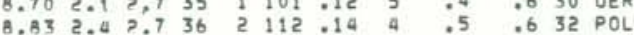

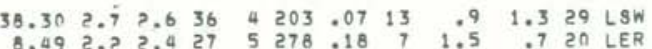

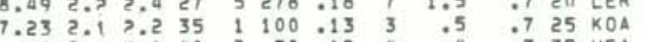

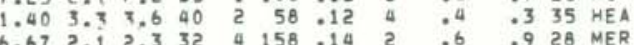

$\begin{array}{llllllllllll}6.47 & 2.0 & 2.0 & 30 & 3 & 138 & .15 & 4 & .5 & 1.2 & 25 & \text { POL } \\ 7.50 & 1.9 & 0.0 & 34 & 3 & 75 & .11 & 5 & .4 & .7 & 31 & \text { UER }\end{array}$

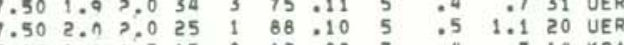

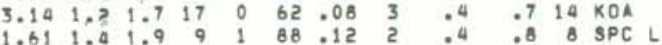

$\begin{array}{rrrrrrrrrrrr}39.29 & 2.4 & 2.6 & 35 & 1 & 75 & : 10 & 4 & .7 & 1.6 & 28 & \text { UER } \\ 7.18 & 2.1 & 1.9 & 21 & 0 & 168 & : 13 & 4 & 1.0 & .6 & 20 & \text { MER }\end{array}$

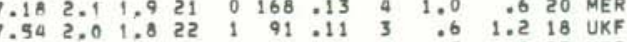
6.412 .520 .828 1 $95.093 \quad$.5 350923 MER $\begin{array}{rlllllllllll}6.15 & 2.0 & 2.3 & 30 & 0 & 123 & .16 & 5 & .6 & 1.1 & 26 & \text { SWR } \\ 7.42 & 1.0 & 0.0 & 33 & 2 & 58 & .17 & 3 & .5 & .8 & 28 & \text { UER }\end{array}$

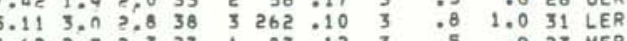

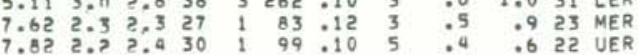

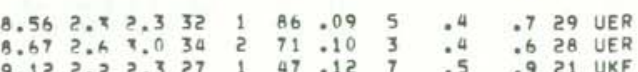




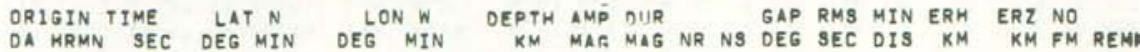

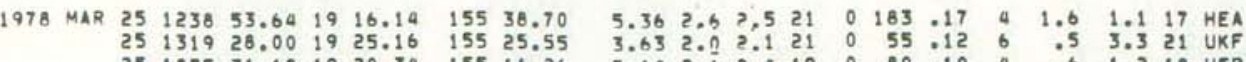
25103531.101920 .3015525 .55 25203834.31 19 $44.91 \quad 1555.08$ $\begin{array}{lllllll}26 & 252 & 03.33 & 19 & 18.51 & 155 & 13.56\end{array}$

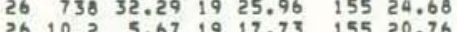
26 $140655.2119 \quad 23.69 \quad 155 \quad 15.02$

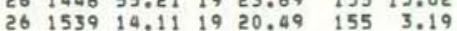

$\begin{array}{lllllll}26 & 1643 & 59.41 & 19 & 24.94 & 155 & 28.28\end{array}$ $\begin{array}{lllllll}26 & 1811 & 3.14 & 19 & 20.03 & 155 & 10.36\end{array}$

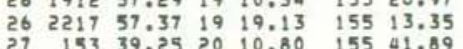

$\begin{array}{lllllll}27 & 211 & 33.72 & 19 & 12.25 & 155 & 27.09\end{array}$ $\begin{array}{lllllll}27 & 311 & 51.72 & 19 & 21.14 & 155 & .89 \\ 27 & 419 & 52.71 & 19 & 20.88 & 155 & 8.00\end{array}$ 27
27 $72938.76 \quad 1920.23 \quad 155 \quad 18.72$ $\begin{array}{lllllll}27 & 1432 & 6.63 & 19 & 19.42 & 155 & 8.24\end{array}$

$\begin{array}{r}27 \\ +\quad 275750.961928 .02 \quad 15450.60 \\ \hline\end{array}$ $\begin{array}{lllllll}28 & 1023 & 48.98 & 19 & 21.37 & 155 & 15.29 \\ 28 & 1315 & 40.13 & 19 & 20.59 & 155 & 11.06\end{array}$

$\begin{array}{lllllll}28 & 1542 & 46.11 & 18 & 49.13 & 155 & 12.05\end{array}$ 15527.04

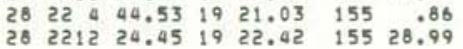

$\begin{array}{lllllll}28 & 2213 & 31.27 & 19 & 21.02 \quad 155 \quad 1.04\end{array}$

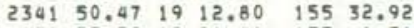
$\begin{array}{llllll}156 & 35.78 & 19 & 21.46 & 155 & 6.56\end{array}$

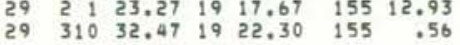
$\begin{array}{lllllll}29 & 1648 & 2.45 & 19 & 22.43 & 155 & 5.70\end{array}$

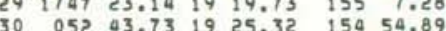

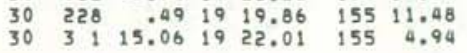
$\begin{array}{llllllll}30 & 5 & 0 & 36.27 & 19 & 17.51 & 155 & 16.20\end{array}$

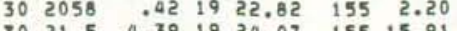

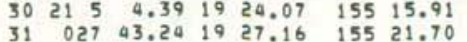

$\begin{array}{lllllll}31 & 217 & 21.44 & 19 & 19.72 \quad 155 & 8.34\end{array}$

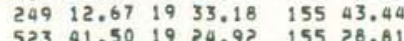
$\begin{array}{rrrrrrrrrrrr}7.14 & 2.1 & 2.4 & 19 & 0 & 80 & -10 & 4 & .6 & 1.2 & 18 & \text { UER } \\ 46.60 & 1.9 & 1.6 & 29 & 5 & 190 & : 10 & 3 & : 9 & 1.0 & 27 & \text { HIL }\end{array}$ $\begin{array}{llllllllllll}7.57 & 2.0 & 1.9 & 27 & 1 & 85 & .12 & 3 & .6 & 1.0 & 19 & \text { POL }\end{array}$ $\begin{array}{lllllllllllll}7.63 & 2.1 & 2.1 & 32 & 0 & 65 & -13 & 8 & .5 & 1.3 & 26 & \text { UKF } \\ 6.62 & 2.0 & 2.0 & 34 & 2 & 125 & 0.17 & 4 & .5 & .9 & 27 & 9 W R\end{array}$

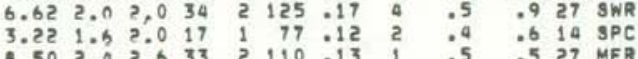
$\begin{array}{lllllllllllll}8.33 & 2.0 & 1.6 & 31 & 3 & 48 & .11 & 5 & .3 & 1.0 & 26 & \text { UKF }\end{array}$

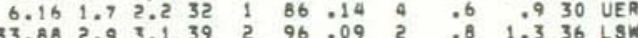

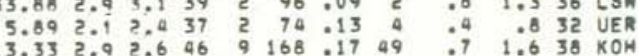

$\begin{array}{llllllllllll}7.54 & 2.1 & 2.3 & 37 & 3 & 121 & .15 & 5 & .6 & .9 & 32 & \text { LSW }\end{array}$

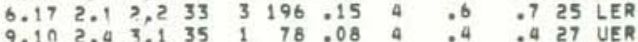
31.742 .72 .436 a 55.0920 .6 .933 DEP $\begin{array}{llllllllllll}.81 & 3.1 & 3.2 & 34 & 1 & 76 & .13 & 4 & .4 & .6 & 30 & \text { HEA }\end{array}$

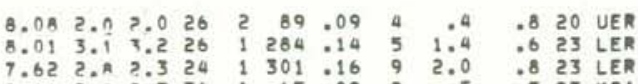

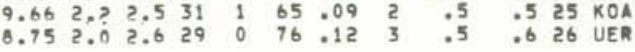
$\begin{array}{llllllllllll}6.34 & 3.5 & 3.2 & 34 & 3 & 292 & .18 & 52 & 5.8 & 37.1 & 32 & \mathrm{PPL}\end{array}$

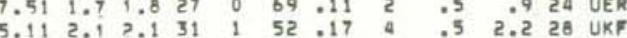

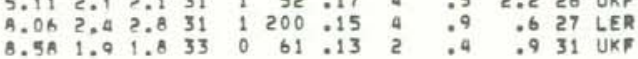
$\begin{array}{lllllllllll}8.05 & 2.1 & 0.2 & 24 & 0 & 197 & .14 & 4 & 1.2 & .620 \text { MER }\end{array}$

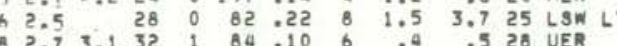

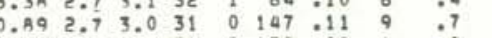

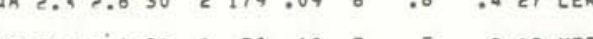

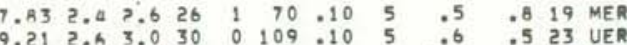

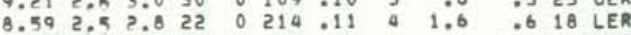

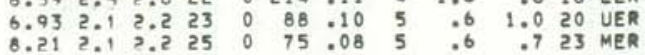
$\begin{array}{lllllllllllll}8.09 & 1.9 & 1.8 & 24 & 0 & 135 & -10 & 5 & .6 & 1.0 & 22 & \mathrm{KOA}\end{array}$

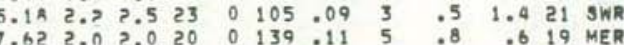

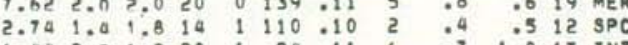

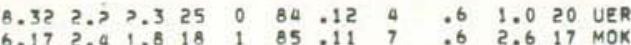

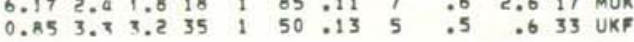

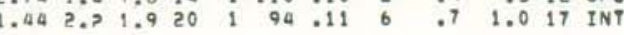

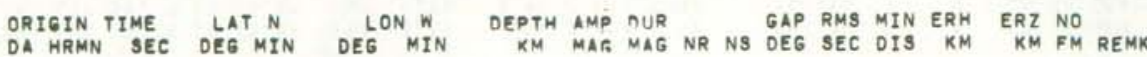

$\begin{array}{llllllll}978 & \text { MAR } 31 & 547 & 2.61 & 19 & 20.05 & 155 & 8.53\end{array}$ $31 \quad 1310 \quad 57.34 \quad 1920.18 \quad 155 \quad 9.10$ 153410.0919 19.11 1551164

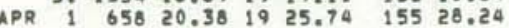
$\begin{array}{lllllll}1 & 13 & 22.64 & 19 & 25.49 & 155 & 28.49 \\ 1 & 23 & 2 & 3.11 & 19 & 19.29 & 155 \\ 2 & 11.86\end{array}$

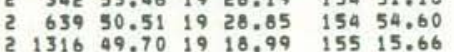

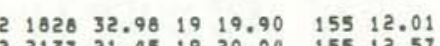
$2137 \quad 21.451920 .04 \quad 155 \quad 12.57$ $\begin{array}{lllllll}3 & 152 & 22.17 & 19 & 39.27 & 155 & 8.56\end{array}$

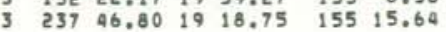

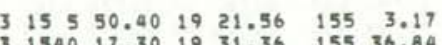
$32252 \quad 54.88 \quad 19 \quad 19.92 \quad 155 \quad 2.91$

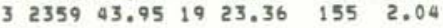

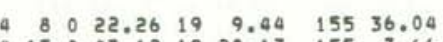
4165911.61 iी 25.06 155 75.66

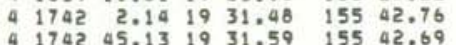
$2205.54 .091923 .69 \quad 15515.98$

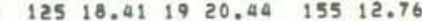
$\begin{array}{lllllll}5 & 427 & 26.34 & 19 & 17.98 & 155 & 21.81 \\ 5 & 522 & 25.33 & 19 & 18.96 & 155 & 12.62\end{array}$

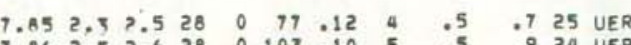

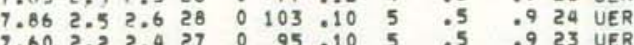

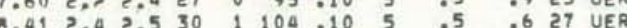

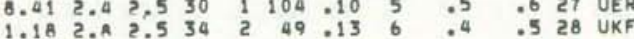

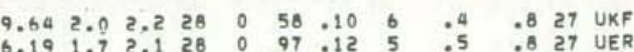

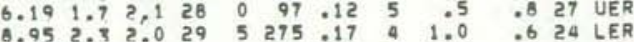

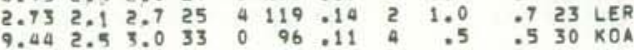
$\begin{array}{lllllllllll}8.80 & 3.3 & 3.4 & 36 & 1 & 83 & .09 & 5 & .4 & .4 & 32 \\ 7 & \text { UER F }\end{array}$

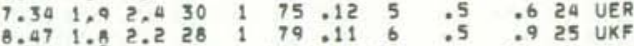

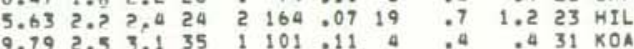
$\begin{array}{lllllllllllll}5.68 & 2.3 & 2.6 & 25 & 1 & 177 & .11 & 6 & .7 & 1.4 & 21 & \text { MER }\end{array}$

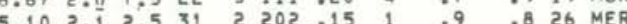

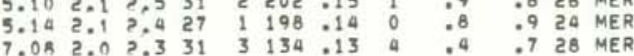

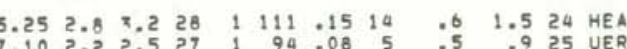
$\begin{array}{lllllllllllll}0.33 & 2.3 & 2.6 & 33 & 2 & 63 & .12 & 6 & .4 & .5 & 27 & \mathrm{UKF} & \end{array}$

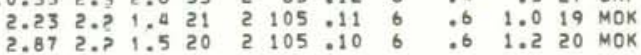

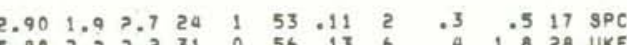

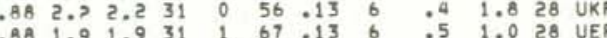

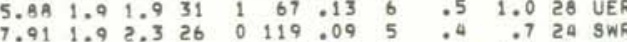

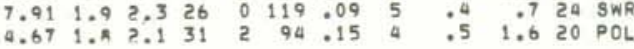

$\begin{array}{llllllllllllllllll}5 & 630 & 40.18 & 19 & 19.87 & 155 & 27.39 & 28.5 A & 2.2 & 2.5 & 32 & 0 & 81 & .10 & 5 & .7 & 1.3 & 25 \\ 5 & \text { HEA }\end{array}$

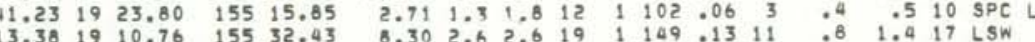

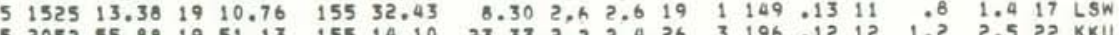

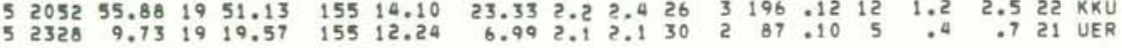

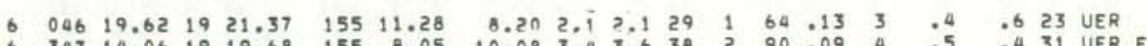

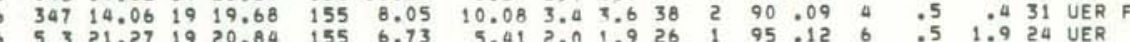

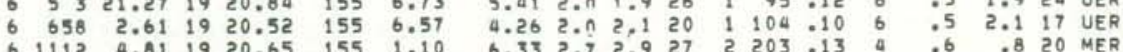

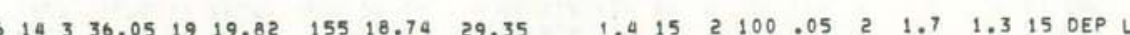

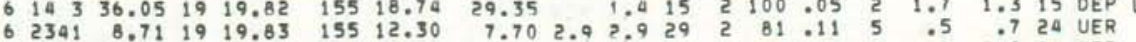

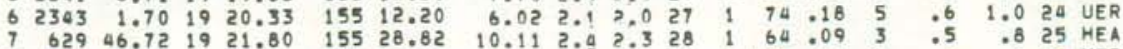

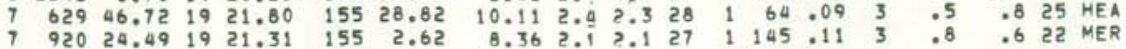

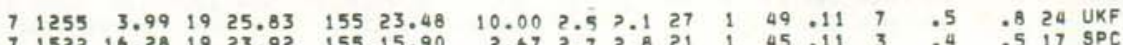

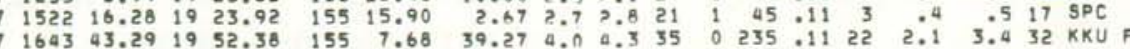




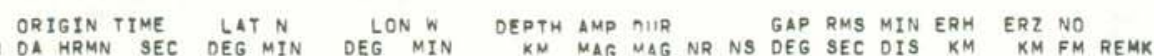

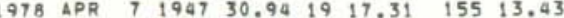

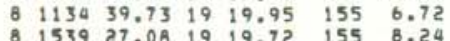
1539
9

$9 \quad 4 \quad 2 \quad 16.16 \quad 1924.49 \quad 154 \quad 57.65$

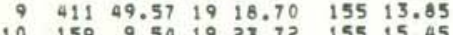
0 159 $30.741923 .72 \quad 15515.45$

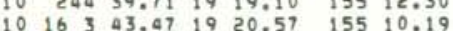

$\begin{array}{rrrrrrr}10 & 2123 & 57.83 & 19 & 29.16 & 155 & 52.84 \\ 11 & 732 & 40.61 & 19 & 12.86 & 155 & 27.43\end{array}$

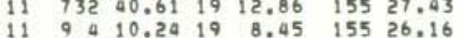

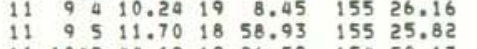
11) 1700 20.30 19 21.56 $155 \quad 1.67$ $\begin{array}{rrrrrrr}11 & 1748 & 22.34 & 19 & 21.56 & 155 & 1.67 \\ 12 & 553 & 41.99 & 19 & 17.61 & 155 & 16.74\end{array}$ $\begin{array}{lllllll}12 & 757 & 2.07 & 19 & 19.14 & 155 & 13.74\end{array}$ $12 \quad 205512.671920 .83 \quad 155 \quad 3.77$

$\begin{array}{lllllll}12 & 2144 & 32.04 & 19 & 21.04 & 155 & 3.31\end{array}$ $4 \begin{array}{lllllll}13 & 010 & 10.55 & 19 & 23.65 & 155 & 15.71 \\ 13 & 537 & 14.17 & 19 & 17.81 & 155 & 23.49\end{array}$ $\begin{array}{rrrrrrr}13 & 631 & 38.97 & 19 & 21.54 & 155 & 2.03 \\ 13 & 1034 & 42.32 & 19 & 25.57 & 155 & 15.65\end{array}$ $\begin{array}{lllllll}13 & 1249 & 59.53 & 19 & 20.85 & 155 & 3.73\end{array}$ 13 2111 $55.161922 .83 \quad 15519.19$

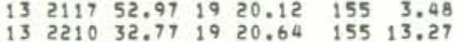

$\begin{array}{lllllll}14 & 030 & 50.88 & 19 & 18.13 & 155 & 45.51\end{array}$ $14512 \quad 33.021923 .74 \quad 15515.83$

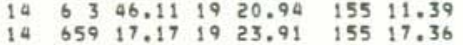
$\begin{array}{llllllll}14 & 8 & 9 & 41.32 & 20 & 5.90 & 155 & 32.77\end{array}$ 14104836.8519 $\begin{array}{lll}29 & 155 & 6.47\end{array}$ $14 \quad 1540 \quad 50.23 \quad 19 \quad 34.72 \quad 155 \quad 40.82$ $\begin{array}{lllllll}14 & 1624 & 29.73 & 19 & 34.28 & 155 & 41.17\end{array}$

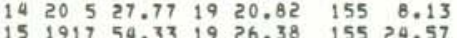

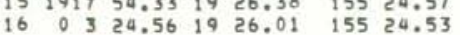
$\begin{array}{lllllll}16 & 920 & .08 & 19 & 21.29 & 155 & 2.30\end{array}$ $\begin{array}{lllllll}16 & 13 & 7.81 & 19 & 24.80 & 155 & 28.69 \\ 16 & 2230 & 6.89 & 19 & 20.54 & 155 & 8.05\end{array}$

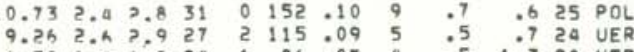

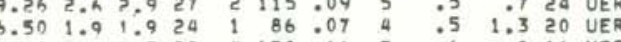

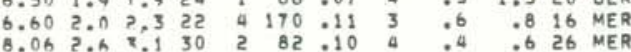
$\begin{array}{rrrrrrrrrrrr}6.44 & 1.9 & 1.4 & 19 & 2 & 186 & .18 & 2 & 1.0 & 1.2 & 15 & \text { LER } \\ 8.49 & 1.7 & 1.7 & 21 & 0 & 94 & .09 & 3 & .5 & .8 & 19 & \text { POL }\end{array}$

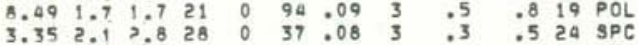

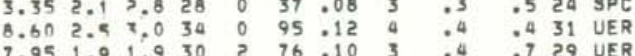

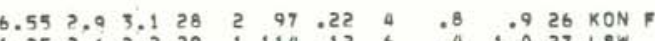

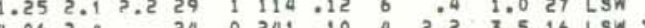

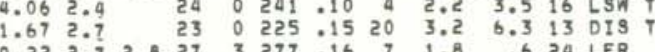

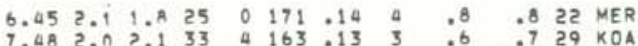
年

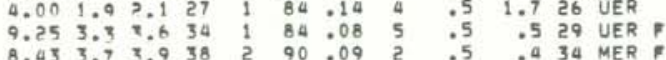
$\begin{array}{llllllllllllllll}3.33 & 2.0 & 1.9 & 27 & 0 & 181 & .16 & 7 & .9 & 3.3 & 23 & \text { MER }\end{array}$

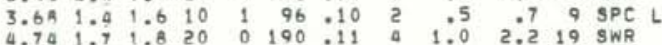
$\begin{array}{lllllllllllll}7.34 & 2.2 & ? .5 & 27 & 1 & 161 & 11 & 3 & .7 & .6 & 24 & \text { MER } \\ 2.54 & 1.7 & 1.9 & 13 & 1 & 126 & .17 & 3 & .5 & .6 & 12 & \text { SPC }\end{array}$ $\begin{array}{lllllllllllll}7.35 & 5.4 & 0.6 & 28 & 1 & 118 & .12 & 2 & .7 & .5 & 25 & \text { MER }\end{array}$

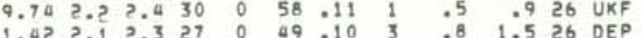

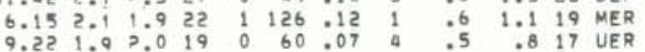

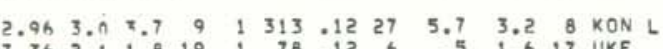

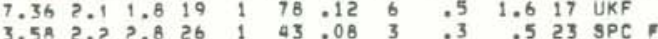

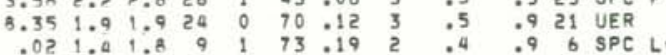

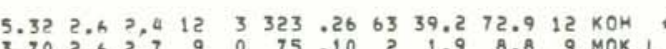
TER

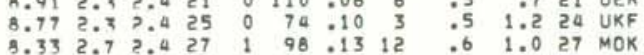

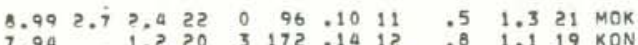
$\begin{array}{llllllllllll}7.32 & 2.1 & 2.3 & 29 & 1 & 77 & .13 & 4 & .6 & .9 & 26 & \text { UER }\end{array}$

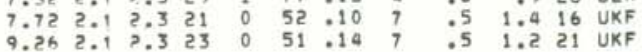
$\begin{array}{llllllllllll}6.45 & 2.1 & 0.2 & 27 & 2 & 157 & .12 & 3 & .6 & .8 & 24 \\ 0 & 4 & \text { MER }\end{array}$

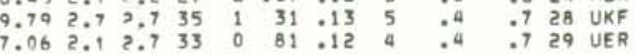

ORIGIN TIME LAT N LON N 1978 APR $17 \quad 0 \quad 3 \quad 17.40 \quad 1925.24 \quad 155 \quad 16.80$

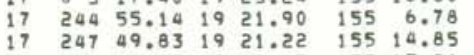

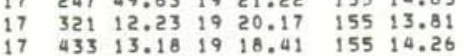
$\begin{array}{lllllll}17 & 1840 & 54.66 & 19 & 21.14 & 155 & 1.44\end{array}$

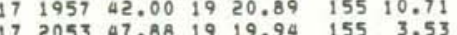

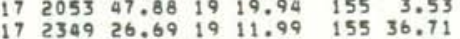
$18 \quad 448 \quad 55.04 \quad 1920.63 \quad 155 \quad 3.86$ $\begin{array}{lllllll}18 & 915 & 14.18 & 19 & 20.59 & 155 & 13.37\end{array}$

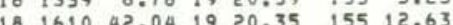

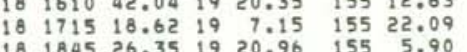
$\begin{array}{lllllll}18 & 2054 & 27.75 & 19 & 19.45 & 155 & 12.11\end{array}$

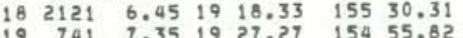

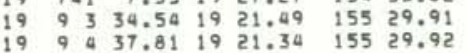
$\begin{array}{lllllll}19 & 1123 & 4.30 & 19 & 32.10 & 155 & 37.26\end{array}$

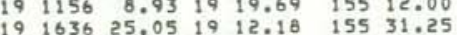

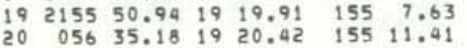

$\begin{array}{lllllll}20 & 145 & 55.62 & 19 & 22.37 & 155 & 6.40\end{array}$ $\begin{array}{lllllll}20 & 145 & 55.62 & 19 & 22.37 & 155 & 6.40 \\ 20 & 453 & 43.66 & 19 & 20.29 & 155 & 3.70\end{array}$

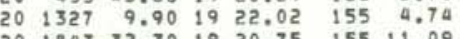
$\begin{array}{lllllll}20 & 1843 & 32.30 & 19 & 20.75 & 155 & 11.09 \\ 20 & 2034 & 33.20 & 19 & 21.41 & 155 & 2.50\end{array}$ $\begin{array}{lllllll}20 & 2349 & 3.53 & 19 & 19.53 & 155 & 11.67\end{array}$ $\begin{array}{lllllll}21 & 145 & 39.55 & 19 & 32.28 & 155 & 28,25\end{array}$ $\begin{array}{lllllll}21 & 414 & 41.39 & 19 & 20.28 & 155 & 17.32 \\ 21 & 838 & 15.55 & 19 & 44.15 & 155 & 34.26\end{array}$ $\begin{array}{lllllll}21 & 913 & 22.23 & 19 & 21.12 & 155 & 2.38\end{array}$ $20.38 \quad 155 \quad 7.42$ $212043 \quad 54.601920 .82 \quad 155 \quad 13.57$ $23 \quad 3,43.46 \quad 19 \quad 20.84 \quad 155 \quad 13.60$

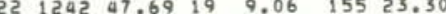
$\begin{array}{lllllll}22 & 1328 & 50.93 & 18 & 51.48 & 155 & 12.80\end{array}$

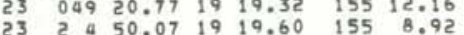

$\begin{array}{lllllll}23 & 726 & 24.49 & 19 & 21.14 & 155 & 15.37\end{array}$ $\begin{array}{lllllll}23 & 947 & 21.68 & 19 & 17.47 & 155 & 21.77 \\ 23 & 1335 & 41.04 & 19 & 18.63 & 155 & 21.47\end{array}$
DEPTH AND RUR
KM MAF MAG NR NS GAP RMS MIN ERH ERE DIS KM KR NO
KM FM REMK

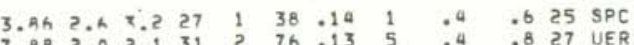

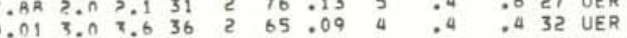

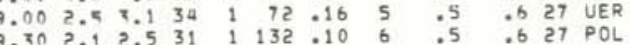

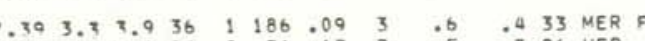
9.04

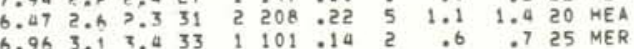
$\begin{array}{lllllllllllll}7.2 R & 2.0 & 2.1 & 30 & 0 & 60 & .11 & 4 & .5 & .8 & 25 & \text { UER }\end{array}$

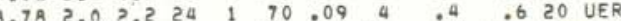
$\begin{array}{lllllllllll}38.37 & 2,1 & 31 & 3 & 192 & .08 & 21 & .9 & 1.5 & 28 & \text { LSW } \\ 7.96,2,2 & 2.4 & 27 & 0 & 99 & .10 & 6 & .6 & .8 & 27 & \text { MER }\end{array}$ \begin{tabular}{llllllllll}
$0.0 n \quad 1.0$ & 2.0 & 26 & 1 & 90 & .10 & 5 & .5 & .6 & 24 \\
\hline & UER
\end{tabular}

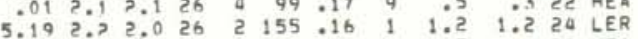

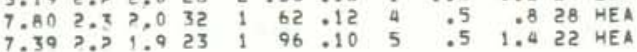
$\begin{array}{lllllllllllll}8.73 \quad 5,4 & 0.1 & 27 & 0 & 114 & .12 & 6 & .5 & .3 & 25 & \text { MOK }\end{array}$

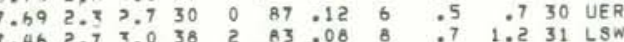

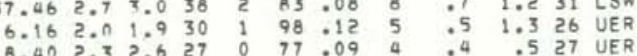
$\begin{array}{lllllllllllll}6.94 & 2.0 & 1.8 & 25 & 0 & 71 & .14 & 6 & .5 & 1.2 & 22 & \text { UER }\end{array}$

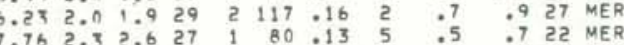

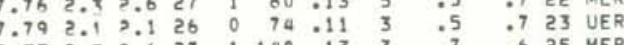

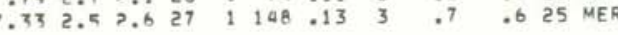
$\begin{array}{llllllllllll}9.50 & 3.0 & 3.2 & 30 & 1 & 94 & .11 & 6 & .5 & .6 & 29 & \text { UER }\end{array}$

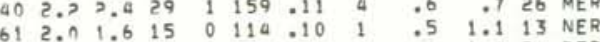

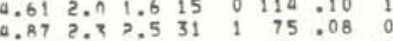
$\begin{array}{llllllllllll}7.42 & 2.4 & 2.5 & 25 & 0 & 157 & .09 & 2 & .7 & .6 & 20 & \text { MER }\end{array}$

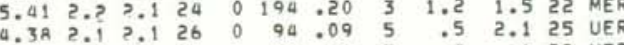

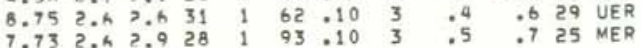
$\begin{array}{lllllllllllll}7.74 & 2,2 & 2.0 & 30 & 1 & 61 & .13 & 3 & .5 & .6 & 28 & \text { UER }\end{array}$

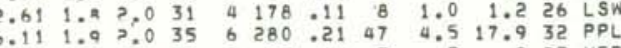

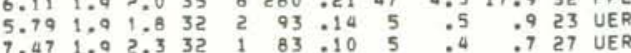

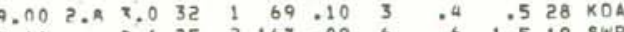
$\begin{array}{llllllllllll}4.16 & 0.1 & 25 & 2 & 167 & .09 & 6 & .6 & 1.5 & 19 & \text { SWR } \\ 4.19 & 1.9 & ? .2 & 27 & 0 & 111 & .13 & 4 & .5 & 1.5 & 25 & \text { SWR }\end{array}$ 


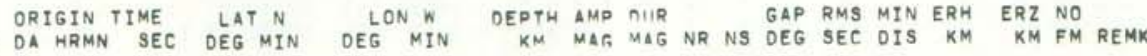
1978 APR $23 \quad 1520 \quad 29.52 \quad 1921.05 \quad 155 \quad 13.29$

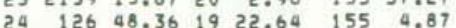

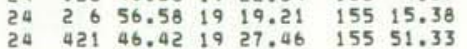
$\begin{array}{lllllll}24 & 838 & 45.70 & 19 & 20.86 & 155 & 2.73 \\ 24 & 843 & 5.89 & 19 & 24.52 & 155 & 25.00\end{array}$

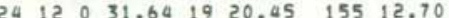

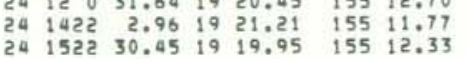
$\begin{array}{llllllll}24 & 16 & 8 & 38.96 & 19 & 21.80 & 155 & 15.27\end{array}$

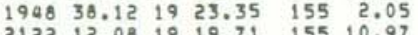
$24 \begin{array}{llllll}2122 & 12.08 & 19 & 19.71 & 155 & 10.97\end{array}$

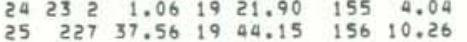
$\begin{array}{lllllll}25 & 354 & 56.66 & 19 & 21.54 & 155 & 1.87\end{array}$ $\begin{array}{lllllll}25 & 820 & 35.73 & 19 & 20.97 & 155 & 5.84\end{array}$ $\begin{array}{lllllll}25 & 1012 & 11.12 & 19 & 21.46 & 155 & 7.23 \\ 25 & 1139 & 55.38 & 10 & 34.7 & 155 & 26.37\end{array}$

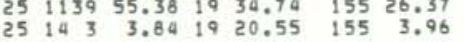

$\begin{array}{lllllllll}25 & 14 & 8 & 34.62 & 19 & 20.63 & 155 & 7.01\end{array}$

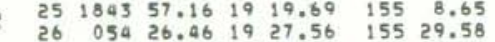
$\begin{array}{lllllll}26 & 128 & 12.61 & 19 & 8.64 & 155 & 30.31\end{array}$

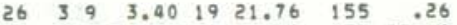
$\begin{array}{lllllll}26 & 348 & 50.20 & 20 & .11 & 155 & 56.48 \\ 26 & 926 & 41.27 & 19 & 21.96 & 155 & 26.78\end{array}$

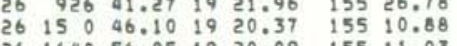
$\begin{array}{llllllll}26 & 178 & 21.71 & 19 & 20.31 & 155 & 11.04 \\ 26 & 1914 & 3.92 & 19 & 28.04 & 154 & 52.28\end{array}$ $262033 \quad 11.011917 .63 \quad 15521.82$ 27.15711 .011917 .635155

$\begin{array}{lrrrrrr}27 & 426 & 21.40 & 19 & 21.03 & 155 & 11.56 \\ 27 & 1517 & 55.92 & 19 & 23.65 & 155 & 16.81\end{array}$

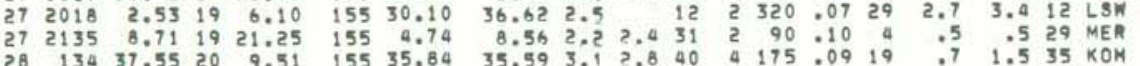

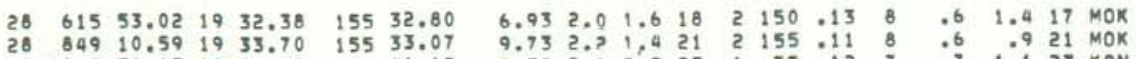

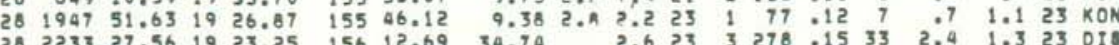

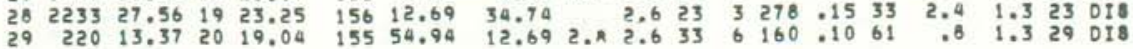
$\begin{array}{llllllllllllllllll}29 & 450 & 40.91 & 19 & 17.15 & 155 & 39.15 & 9.68 & 2.5 & 2.3 & 30 & 2 & 82 & .15 & 6 & .6 & .9 & 27 \\ 29 & 524 & 57.21 & 19 & 19.37 & 155 & 11.75 & 7.14 & 2.2 & 2.6 & 34 & 2 & 96 & .13 & 5 & .5 & .7 & 30 \\ \text { UER }\end{array}$ YeAR MON ORIGIN TIME LARM GEC DEG MIN DEG MON WIN 1978 APR $29 \quad 155929.48 \quad 19 \quad 11.81 \quad 155 \quad 41.07$

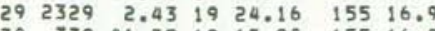
$30 \quad 339 \quad 41.75 \quad 19 \quad 17.98 \quad 155 \quad 16.26$ $\begin{array}{llllllll}30 & 6 & 1 & 5.68 & 19 & 19.15 & 155 & 11.30\end{array}$ $\begin{array}{llllllll}30 & 15 & 8 & 18.45 & 19 & 20.32 & 155 & 11.78\end{array}$ $\begin{array}{lllllll}30 & 1550 & 41.49 & 19 & 19.90 & 155 & 15.38 \\ 30 & 2016 & 17.97 & 19 & 20.31 & 155 & 11.76\end{array}$ $\begin{array}{lllllll}30 & 2148 & 17.09 & 19 & 29.13 & 155 & 49.60\end{array}$ $30 \quad 2148536.09$

MAY $1 \quad 423 \quad 46.57 \quad 19 \quad 23.33 \quad 155 \quad 16.8$

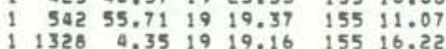

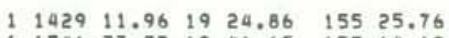
$1 \quad 1546 \quad 37.37 \quad 19 \quad 46.15 \quad 155 \quad 14.12$ $\begin{array}{lrrrrrr}1 & 1659 & 21.56 & 19 & 18.12 & 155 & 15.46 \\ 2 & 540 & 28.24 & 19 & 21.13 & 155 & 7.28\end{array}$ $\begin{array}{lrrrrrr}2 & 540 & 28.24 & 19 & 21.13 & 155 & 7.28 \\ 2 & 2239 & 35.93 & 19 & 21.66 & 155 & 14.12\end{array}$

$3 \quad 729 \quad 6.4019 \quad 17.49 \quad 15529.96$

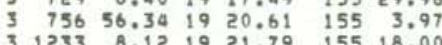

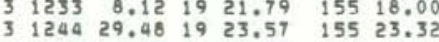
$31752 \quad 27.791921 .64 \quad 155 \quad 4.68$
0

$\begin{array}{lrrrrrr}3 & 2223 & 53.17 & 19 & 16.08 & 155 & 6.09 \\ 4 & 316 & 26.79 & 19 & 27.027 & 155 & 23.05\end{array}$

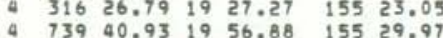

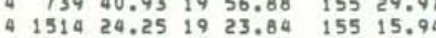

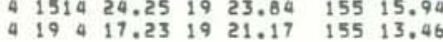

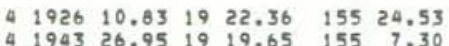

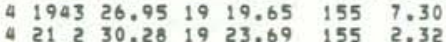

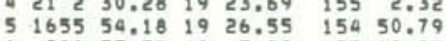
$\begin{array}{rrrrrrr}5 & 1655 & 54.18 & 19 & 26.55 & 154 & 50.79 \\ 6 & 220 & 35.57 & 19 & 5.99 & 155 & 28.25\end{array}$

$\begin{array}{lllllll}6 & 316 & 49.62 & 19 & 55.15 & 155 & 20.42 \\ 6 & 812 & 39.39 & 19 & 24.51 & 155 & 26.25\end{array}$

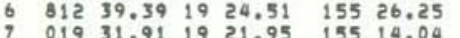
7
7 $53621.721920 .65 \quad 15512.59$

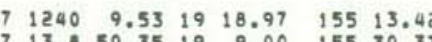
$713350.3519 \quad 9.00 \quad 155 \quad 30.37$ 1 $01143.321923 .57 \quad 155 \quad 4.07$ $1432 \quad 57.05 \quad 19 \quad 47.55 \quad 155 \quad 1.71$
0 $9437 \quad 16.55 \quad 1920.12 \quad 155 \quad 6.46$

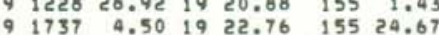

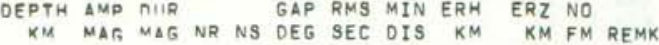
$\begin{array}{llllllllllllll}5.76 & 1.6 & 1.8 & 23 & 4 & 148 & .24 & 9 & 1.1 & 2.8 & 11 & \text { HEA } & \end{array}$

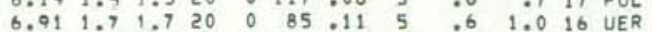
$\begin{array}{llllllllllllll}1.56 & 1.4 & 1.9 & 7 & 1 & 110 & 110 & 1 & .4 & .6 & 6 & \mathrm{SPC} \\ 7.13 & 7.0 & 1.8 & 23 & 1 & 158 & .09 & 4 & .0 & .0 & 17 & \mathrm{KOA}\end{array}$

$\begin{array}{llllllllllll}7.33 & 2.2 & 2.1 & 29 & 1 & 105 & .12 & 5 & .5 & .7 & 24 & \\ 7 & \text { UER }\end{array}$

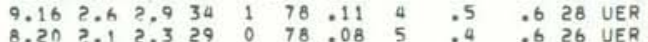

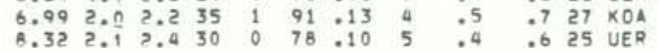
$\begin{array}{lllllllllll}7.37 & 1.9 & 25 & 2 & 217 & .17 & 7 & 1.3 & .9 & 21 & \text { KON }\end{array}$

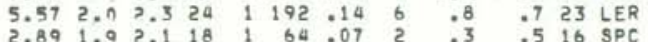

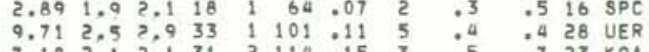
$.03 \quad 5.520 .530255 \quad 16 \quad 5$

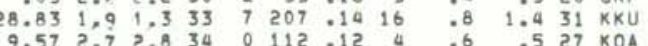

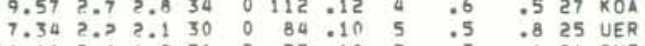
$\begin{array}{llllllllllll}7.88 & 3.3 & 7.5 & 36 & 1 & 75.13 & 10 & .5 & .8 & 33 & \text { HEA F }\end{array}$

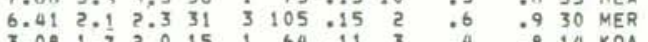

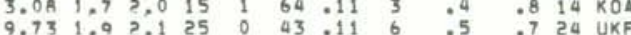
7.102 .02 .126 O 80.11 a .5 .8 24 MER

$\begin{array}{llllllllllll}9.95 & 2.2 & 2.3 & 27 & 1 & 225 & 1.4 & 9 & 1.0 & .7 & 25 & \text { POL } \\ 8.15 & 2.0 & 0.0 & 22 & 0 & 75 & 113 & 5 & 5 & 1.3 & 21 & \text { UKF }\end{array}$

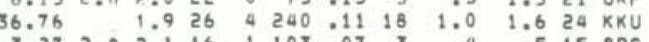

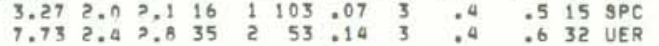

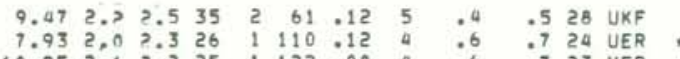
10.95 2.1 2.2 25 1 122.08 4 .601 .7 23 MER

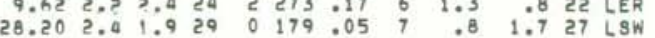

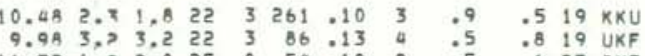

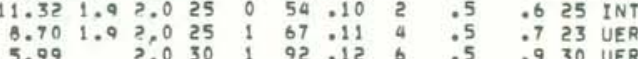
$\begin{array}{rrrrrrrrrrrr}6.31 & 2.5 & 2.8 & 39 & 3 & 75 & .18 & 4 & .5 & .9 & 36 & \text { POL } \\ 9.66 & 2.4 & 2.5 & 27 & 2 & 145 & .11 & 5 & .6 & .9 & 25 & \mathrm{LSW}\end{array}$

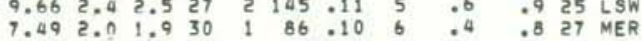

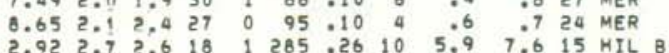

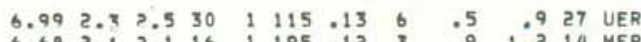

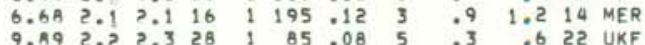




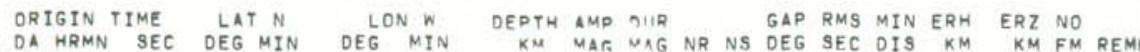

1978 MAY $9 \begin{array}{lllll}1846 & 41.16 \quad 19 & 20.17 \quad 155 \quad 12.02\end{array}$

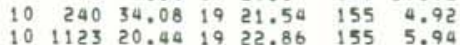
$101245 \quad 32.801926 .46 \quad 15521.35$ $\begin{array}{lllllll}10 & 1423 \quad 57.94 \quad 19 & 19.46 \quad 155 \quad 6.68\end{array}$ $10142921.461918 .96 \quad 155 \quad 6.75$ $\begin{array}{lllllll}11 & 128 & 46.53 & 19 & 24.94 & 154 & 54.03 \\ 12 & 458 & 26.84 & 19 & 20.70 & 155 & 10.90\end{array}$

$\begin{array}{lllllll}12 & 511 & 6.14 & 19 & 21.12 & 155 & 2.41\end{array}$ $1253555.951913 .94 \quad 15535.44$

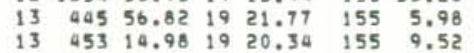

$\begin{array}{llllllll}13 & 732 & 12.44 & 19 & 23.53 & 155 & 15.98\end{array}$ $13 \quad 74950.931923 .83 \quad 15515.94$ $13 \quad 9745.73 \quad 1923.75 \quad 15516.00$ $\begin{array}{rrrrrrr}14 & 321 & 52.92 & 19 & 19.29 & 155 & 11.65 \\ 14 & 1039 & 36.79 & 19 & 24.84 & 155 & 16.04\end{array}$ $\begin{array}{lllllll}14 & 1746 & 1.00 & 19 & 19.7 & 155 & 10.85 \\ 15 & 147 & 12.11 & 19 & 26.01 & 155 & 50.95\end{array}$

$15 \quad 336 \quad 41.27 \quad 19 \quad 21.85 \quad 155 \quad 5.78$ $15 \quad 301 \quad 46.37 \quad 1920.95 \quad 155 \quad 5.80$ $\begin{array}{lllllll}15 & 1337 & 14.64 & 19 & 19.83 & 155 & 8.48\end{array}$

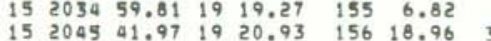

$\begin{array}{lllllll}16 & 418 & 23.84 & 19 & 20.11 & 155 & 11.40\end{array}$

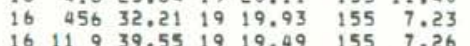

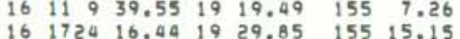

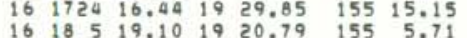

$\begin{array}{lllllll}16 & 1951 & 50.27 & 19 & 19.95 & 155 & 11.51 \\ 16 & 2235 & 31.84 & 19 & 20.49 & 155 & 12.21\end{array}$

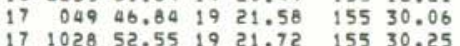

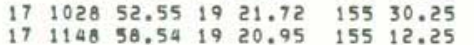

$\begin{array}{lllllll}17 & 1658 & 25.87 & 19 & 20.58 & 155 & 8.00 \\ 17 & 2213 & 30.02 & 19 & 27.51 & 155 & 28.75\end{array}$

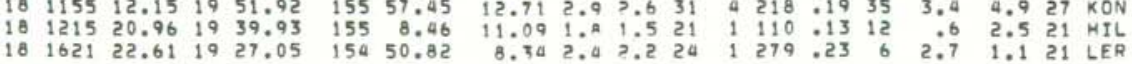
$\begin{array}{rrrrrrrrrrrrrrrrrrrr}18 & 1814 & 18.07 & 19 & 17.46 & 155 & 13.47 & 9.99 & 2.1 & 2.1 & 28 & 0 & 149 & .11 & 8 & .6 & .6 & 26 & P O L \\ 18 & 1828 & 9.08 & 19 & 15.95 & 155 & 13.61 & 10.34 & 2.0 & 2.6 & 33 & 3 & 161 & .13 & 10 & .6 & .5 & 28 & \text { POL }\end{array}$

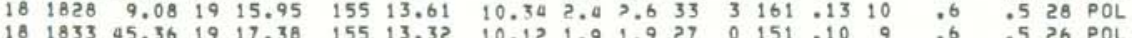

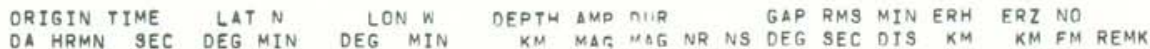

1978 MAY $1820728.401920 .53 \quad 155 \quad 6.48$

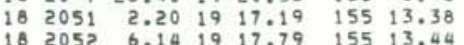

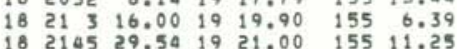

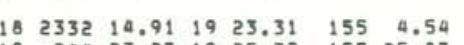
$19 \quad 24423.2719 \quad 25.32 \quad 155 \quad 25.87$

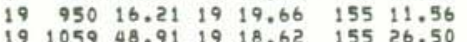

$\begin{array}{lllllll}19 & 129 & 8.13 \quad 19 & 6.13 & 155 & 34.68\end{array}$ $\begin{array}{rrrrrrr}19 & 129 & 8.13 & 19 & 6.13 & 155 & 34.68 \\ 19 & 1453 & 44.39 & 19 & 20.14 & 155 & 11.75\end{array}$ $20 \quad 2950.291926 .85 \quad 15451.73$ $\begin{array}{llrllll}20 & 243 & 9.06 & 19 & 20.64 & 155 & 7.80 \\ 20 & 344 & 52.34 & 19 & 23.76 & 155 & 16.92\end{array}$ $\begin{array}{llllllll}20 & 421 & 25.27 & 19 & 21.60 & 155 & 5.98\end{array}$ $20 \quad 1352 \quad 10.801910 .41 \quad 155 \quad 20.66$

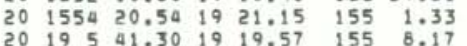

$\begin{array}{llllllll}21 & 4 & 8 & 22.00 & 19 & 19.73 \quad 155 & 12.26\end{array}$ 21 131751.60 19 19.70 155 13.02

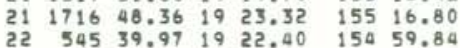
$\begin{array}{lllllll}22 & 819 & 18.21 & 19 & 25.20 & 155 & 25.16\end{array}$ $22 \quad 82011.64 \quad 1950.15 \quad 155 \quad 15.60$ $22 \quad 1257 \quad 38.28 \quad 19 \quad 14.72 \quad 155 \quad 35.62$

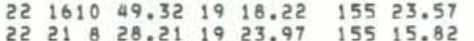

$\begin{array}{llllllll}22 & 21 & 9 & 26.70 & 19 & 16.58 & 155 & 13.46\end{array}$

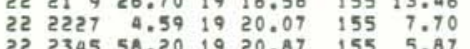
$222345 \quad 58.20 \quad 1920.87 \quad 155 \quad 5.87$

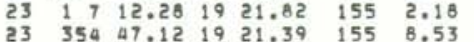

$23 \quad 7940.14 \quad 19 \quad 19.23 \quad 155 \quad 13.90$

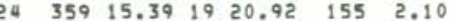
$\begin{array}{rrrrrrr}24 & 438 & 46.64 & 19 & 25.45 & 155 & 25.71 \\ 24 & 1222 & 19.13 & 19 & 23.52 & 155 & 15.01\end{array}$ $24165252.981921 .06 \quad 15512.79$ $\begin{array}{rrrrrrr}24 & 1939 & 47.83 & 19 & 21.17 & 155 & 12.80 \\ 25 & 012 & 13.46 & 19 & 18.40 & 155 & 13.60\end{array}$

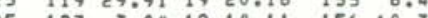
$25 \quad 80 \quad 30.97 \quad 1926.25$ is 24.35 $\begin{array}{lllllll}25 & 945 & 47.39 & 19 & 18.93 & 155 & 22.09\end{array}$

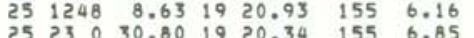

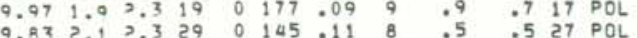

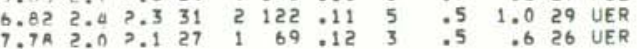

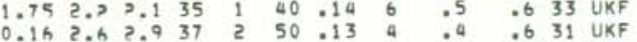

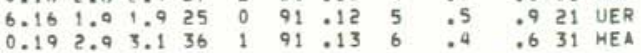

$\begin{array}{llllllllllll}0.02 & 2.0 & 2.5 & 17 & 1 & 153 & .09 & 14 & 1.8 & 2.6 & 4 & \text { MEA }\end{array}$ 5.71 . $\begin{array}{llllllllllll}7.49 & 1.9 & 1.7 & 26 & 2 & 85.09 & 4 & .4 & .9 & 19 & \text { UER } \\ 2.87 & 1.5 & 1.0 & 17 & 1 & 65 & .10 & 2 & .3 & .5 & 12 & \text { SPC }\end{array}$ $\begin{array}{llllllllllll}7.27 & 2.1 & 2.6 & 31 & 3 & 84 & .09 & 6 & .4 & .9 & 25 & \text { MER }\end{array}$

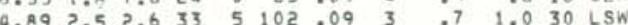

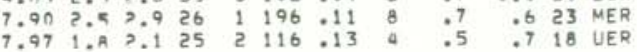
$\begin{array}{lllllllllll}5.46 & 7.1 & 2.1 & 29 & 3 & 83 & .15 & 5 & .5 & 1.0 & 20 \\ \text { UER }\end{array}$

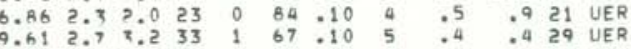

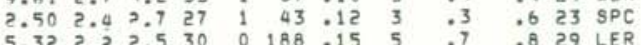
$6.5 \mathrm{~A}, 1.930,48.16,9.4,1.626$ UKF

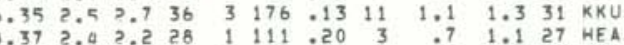

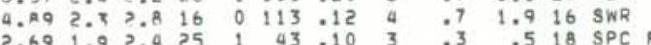

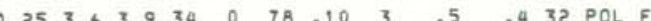

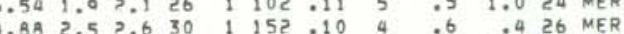

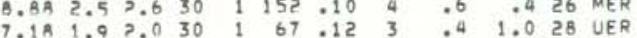

$\begin{array}{lllllllllllll}. R 7 & 2.0 & 2.2 & 30 & 0 & 65 & 113 & 4 & .5 & .6 & 28 & \text { UER } \\ .48 & 3 & 5 & 5 & 37 & 2 & 174 & 11 & 2 & .6 & .5 & 37 & \text { MER }\end{array}$

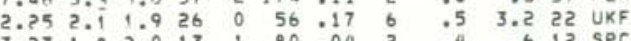

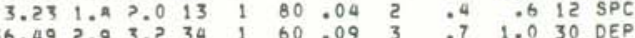
$\begin{array}{rrrrrrrrrrrr}37.99 & 3.0 & 7.1 & 34 & 0 & 60 & 10 & 3 & .7 & 1.6 & 30 & \text { DEP } \\ 9.54 & 7.9 & 0.1 & 30 & 2 & 73 & .12 & 3 & .5 & .5 & 26 & \text { POL }\end{array}$

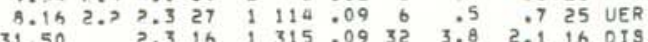

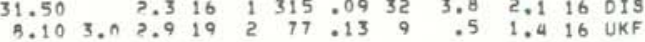
$\begin{array}{llllllllllll}0.02 & 2.1 & 2.3 & 20 & 0 & 149 & .11 & 3 & .7 & 1.2 & 19 & \text { SWR }\end{array}$

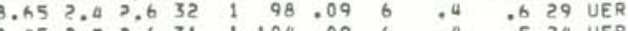

$\begin{array}{llllllllllll}7.4 \times 2.0 & 2.5 & 30 & 2 & 104 & -10 & 6 & .4 & .7 & 27 \\ 9 & \text { UER }\end{array}$

$\begin{array}{llllllllllll}7.37 & 2.5 & 2.8 & 34 & 2 & 84 & .10 & 2 & .4 & .7 & 32 & \text { MER }\end{array}$ $\begin{array}{lllllllll}0.5 & 31 & \text { UER }\end{array}$ 
YEAR MON ORIGIN TIME HRMN SEC DEAT MIN DEG MIN DEPTH AMP NUR MAR GAP RMS MIN ERH ERZ NO

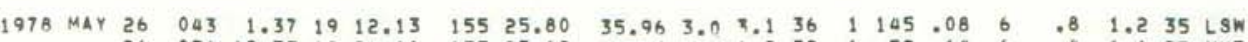

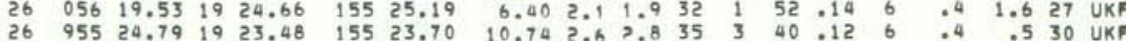

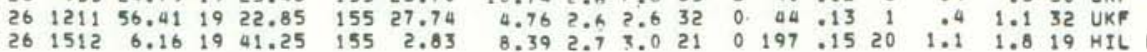
$\begin{array}{lllllllllllllllllll}26 & 1910 & 9.05 & 19 & 19.92 & 155 & 6.75 & 8.87 & 2.4 & 9.4 & 32 & 1 & 115 & .09 & 5 & .4 & .5 & 30 & \text { UER }\end{array}$ $0.432,5301126.0955050$

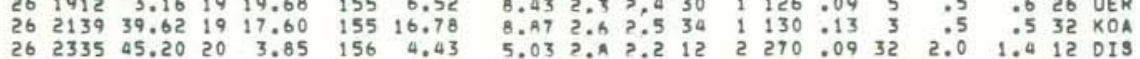

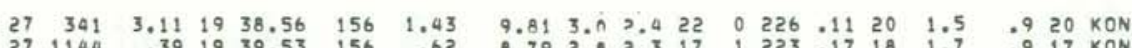

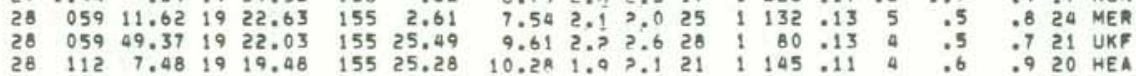
$\begin{array}{lllllllllllllllllll}28 & 648 & 4.47 & 19 & 20.35 & 155 & 5.76 & 7.90 & 2.1 & 2.1 & 26 & 1 & 116 & .10 & 5 & .5 & .8 & 25 & \text { MER }\end{array}$

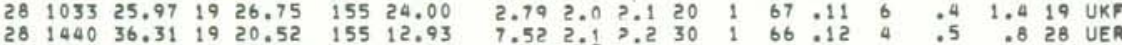

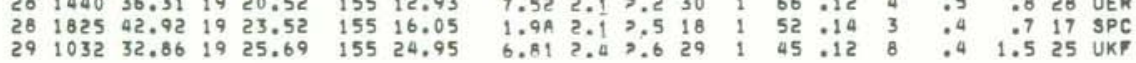
$\begin{array}{lllllllllllllllllll}29 & 1528 & 4.20 & 19 & 26.36 & 155 & 21.44 & 7.83 & 2.5 & 0.6 & 35 & 3 & 41 & .17 & 6 & .4 & .9 & 31 & \text { UKF }\end{array}$ $\begin{array}{lllllll}29 & 17 & 28.18 & 19 & 19.76 & 155 & 13.39\end{array}$ $2917533.271919 .11 \quad 15511.66$

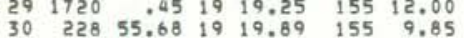

$\begin{array}{lllllll}30 & 1257 & 39.93 & 19 & 19.04 & 155 & 12.05\end{array}$

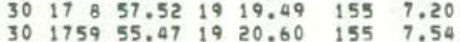

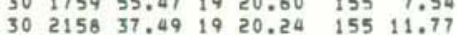
$314^{4} \quad 46.13 \quad 1921.49 \quad 155 \quad 15.36$

$\begin{array}{lllllll}31 & 434 & 19.89 & 19 & 19.98 & 155 & 11.40 \\ 31 & 750 & 36.55 & 18 & 48.95 & 155 & 9.99\end{array}$ $31 \quad 1433 \quad 44.281920 .49 \quad 15513.23$

$31.612 \quad 36.50$ 19 21.25 155 12.26

$\begin{array}{lllllll}31 & 1912 & 9.65 & 19 & 19.41 & 155 & 18.64 \\ 31 & 1915 & 30.22 & 19 & 19.54 & 155 & 18.59\end{array}$ 31191655.471919 .57 155 18.86

JUN 1 1 $119 \quad 37.94 \quad 1926.31 \quad 15527.82$

$\begin{array}{lllllll}1 & 154 & 35.03 & 19 & 26.04 & 155 & 24.35 \\ 1 & 530 & 27.42 & 19 & 21.01 & 155 & 13.20\end{array}$

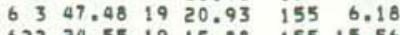

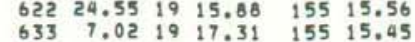

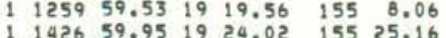

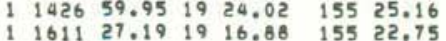

$\begin{array}{rrrrrrrrrrrr}7.83 & 2.5 & 0.6 & 35 & 3 & 41 & .17 & 6 & .4 & .9 & 31 & \text { UKF } \\ 5.23 & 1.0 & 1.9 & 33 & 1 & 67 & .19 & 5 & : 6 & 1.3 & 29 & \text { UER } \\ 0.32 & 0.2 & .3 & 33 & 1 & 104 & .15 & 5 & .5 & 99 & 29 & \text { UER }\end{array}$

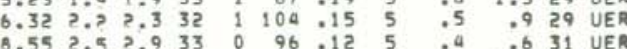

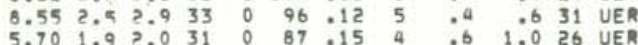
$\begin{array}{llllllllllll}6.62 & 2.1 & 2.2 & 30 & 1 & 101 & .16 & 4 & .6 & .9 & 25 & \text { UER } \\ 7.70 & 2.2 & 2.6 & 31 & 1 & 116 & 010 & 4 & .5 & .8 & 29 & \text { UER }\end{array}$

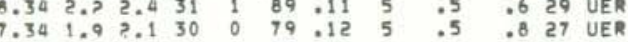

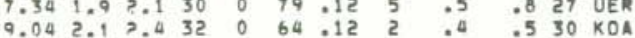

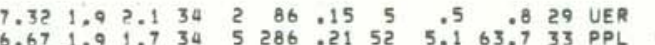

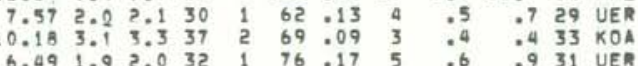

$\begin{array}{llllllllllll}5.93 & 2.1 & 2.3 & 28 & 1 & 77 & .14 & 3 & .5 & .8 & 27 & K O A \\ 5.92 & 2.7 & 2.4 & 30 & 1 & 74 & .11 & 2 & .5 & .8 & 28 & K O A\end{array}$

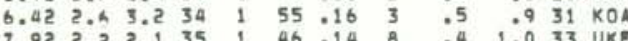

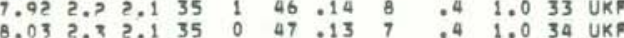

$\begin{array}{llllllllllllll}0.83 & 2.9 & 2.3 & 36 & 0 & 31 & .16 & 7 & .5 & .9 & 33 & \text { UKF }\end{array}$

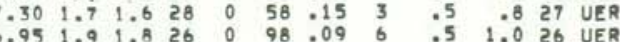

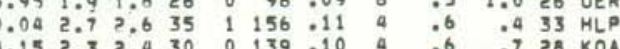

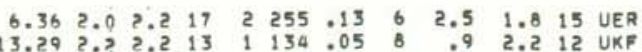

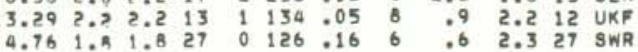

ORIGIN TIME
OA HRMN SAT N
SEEG MIN OON WW

978 JUN $119440.23 \quad 1927.04 \quad 15529.8$ 203128.13
132 23.05 i9 $20.07 \quad 155 \quad 6.20$ 45952.971919 .78
2

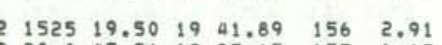

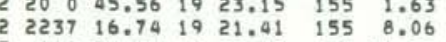

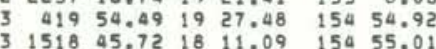

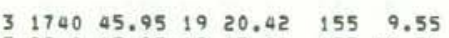

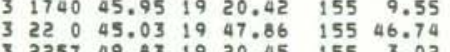

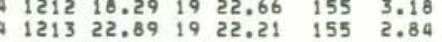

$\begin{array}{lllllll}4 & 1235 & 59.20 & 19 & 20.89 & 155 & 6.01\end{array}$ $41840 \quad 43.33 \quad 19 \quad 19.75 \quad 155 \quad 11.02$ $\begin{array}{rrrrrrr}5 & 29 & 32.53 & 19 & 19.38 & 155 & 7.16 \\ 5 & 1425 & 6.20 & 19 & 23.84 & 155 & 15.85\end{array}$

$\begin{array}{lllllll}5 & 1531 & 22.26 & 19 & 19.63 & 155 & 7.71\end{array}$

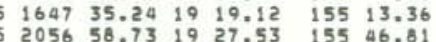
$2132 \quad 5.291931 .62 \quad 15528$.

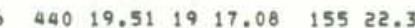
$1021 \quad 53.56 \quad 19 \quad 23.67 \quad 155 \quad 15.87$
6

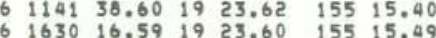
$6232133.82 \quad 1923.26 \quad 155 \quad 16.70$

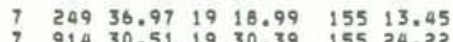
$91430.51 \quad 1930.39$
$755 \quad 24.22$

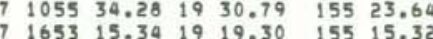
$182023.27 \quad 1920.89 \quad 15511.51$

$\begin{array}{lllllll}7 & 1827 & 57.59 & 19 & 19.89 & 155 & 7.89\end{array}$

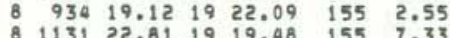
$\begin{array}{lllllll}8 & 1131 & 22.81 & 19 & 19.48 & 155 & 7.33 \\ 8 & 1527 & 47.11 & 19 & 42.75 & 155 & 3.69\end{array}$ $\begin{array}{lllllll}8 & 1527 & 47.11 & 19 & 42.75 & 155 & 3.69 \\ 8 & 1618 & 18.05 & 19 & 20.24 & 155 & 6.68\end{array}$

$\begin{array}{lllllll}8 & 1625 & 41.51 & 19 & 17.29 & 155 & 21.41 \\ 8 & 2054 & 14.517 & 19 & 18.75 & 155 & 7.14\end{array}$

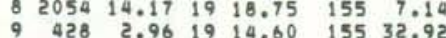

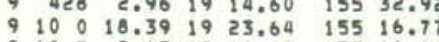

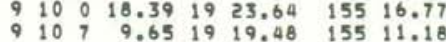

$\begin{array}{lllllll}9 & 1055 & 39.97 & 19 & 18.46 & 155 & 14.96 \\ 9 & 1551 & 25.56 & 19 & 21.31 & 155 & 28.39\end{array}$

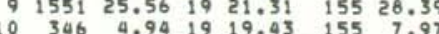

DEPTH AMP PUR
KM MAT MAG NR NS GAP RMS MIN ERH ERZ NO
DEC OIS KM KM FM REMK $\begin{array}{rrrrrrrrrrrr}9.37 & 2.4 & 2.3 & 32 & 0 & 72 & .11 & 9 & .4 & .7 & 32 & \text { UKF } \\ 7.85 & 2.0 & 9.1 & 30 & 2 & 102 & .13 & 5 & .5 & .9 & 27 & \text { UER }\end{array}$

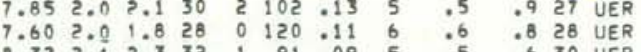

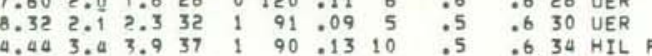
$\begin{array}{llllllllllll}7.89 & 5.9 & 7.0 & 23 & 0 & 283 & .14 & 22 & 3.4 & 1.0 & 19 & \mathrm{KON}\end{array}$

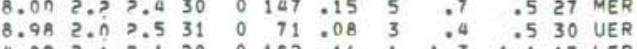

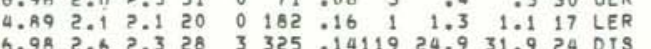

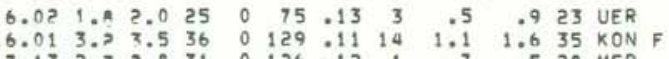

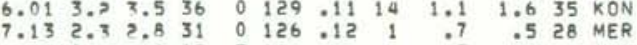
$\begin{array}{llllllllllll}7.39 & 2.0 & 2.0 & 27 & 3 & 116 & .14 & 4 & .5 & .5 & 25 & \text { MER } \\ 9.03 & 2.7 & 2.0 & 34 & 3 & 130 & .12 & 4 & .4 & .5 & 29 & \text { MER }\end{array}$ $\begin{array}{llllllllllllll}8.61 & 2.1 & 3.0 & 33 & 1 & 100 & .11 & 6 & .4 & .5 & 30 & \text { UER }\end{array}$

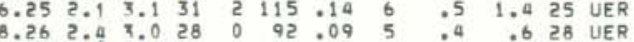

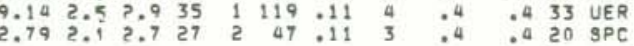
$\begin{array}{llllllllllll}4.21 & 1.0 & 2.0 & 28 & 1 & 100 & .12 & 4 & .5 & 1.9 & 26 & \text { UER }\end{array}$

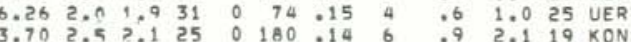
$\begin{array}{llllllllllll}3.64 & 2.1 & 2.0 & 25 & 0 & 97 & .13 & 1 & .4 & .8 & 21 & \text { NER } \\ 7.20 & 1.7 & 1.8 & 30 & 0 & 92 & .11 & 5 & .5 & .0 & 26 & \text { UER }\end{array}$

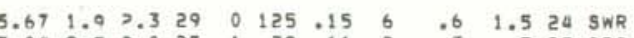

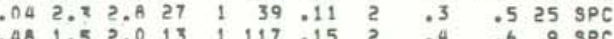

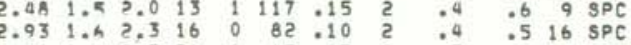
$.611 .92 .326 \quad 1 \quad 45.13 \quad 2 \quad .3 \quad .518 \mathrm{SPC}$

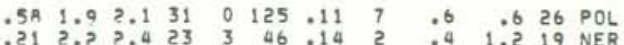

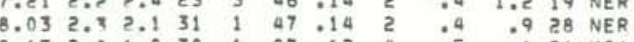

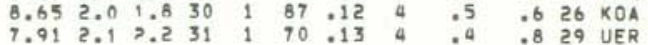
$\begin{array}{lllllllllll}7.11 & 2.2 & 2.2 & 31 & 0 & 93 & .11 & 5 & .5 & .9 & 29 \\ 9 & .9 & \text { UER }\end{array}$

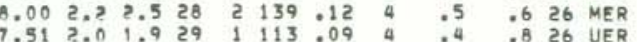

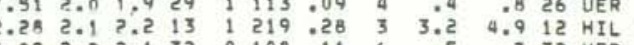
$\begin{array}{llllllllllll}8.56 & 2.1 & 2.8 & 29 & 1 & 133 & .10 & 5 & .5 & .7 & 28 & \text { SWR }\end{array}$

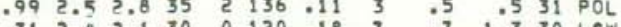

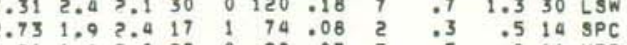

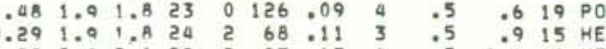




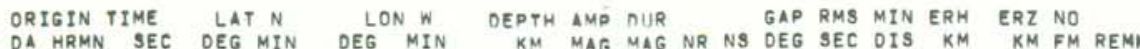

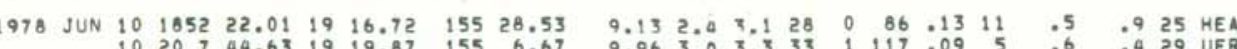
$10207 \quad 44.63 \quad 19 \quad 19.87 \quad 155 \quad 6.67$

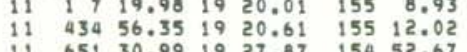
$\begin{array}{lllllll}11 & 934 & 4.96 & 19 & 20.91 & 155 & 6.52\end{array}$ $\begin{array}{rrrrrrr}11 & 1248 & 36.64 & 19 & 17.07 & 155 & 27.83 \\ 12 & 240 & .62 & 19 & 22.29 & 155 & 25.16\end{array}$

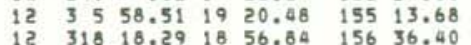
$\begin{array}{lllllll}12 & 836 & 29.61 & 19 & 25.40 & 155 & 24.58\end{array}$

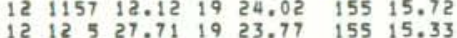

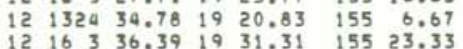
$\begin{array}{lllllll}12 & 1626 & 43.12 & 19 & 19.70 & 155 & 11.75\end{array}$ $121826 \quad 53.321923 .64 \quad 15516.69$

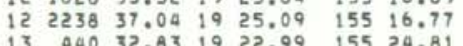

$\begin{array}{lllllll}13 & 442 & 40.84 & 19 & 24.83 & 155 & 24.99\end{array}$ 13
13 $\quad 654 \quad 1.151920 .47 \quad 155 \quad 3.21$ $\begin{array}{rrrrrrr}13 & 710 & 7.08 & 19 & 18.77 & 155 & 13.60 \\ 13 & 843 & 18.67 & 19 & 19.78 & 155 & 2.83\end{array}$

$\begin{array}{lllllll}13 & 1941 & 11.48 & 19 & 20.57 & 155 & 3.49\end{array}$ $14 \quad 64238.44 \quad 1921.05 \quad 155 \quad 6.21$ $14 \quad 720 \quad 19.49 \quad 19 \quad 17.52 \quad 155 \quad 13.54$

$14215359.10 \quad 1920.26 \quad 155 \quad 3.05$ $1422 \quad 5 \quad 40.81 \quad 1923.62 \quad 155 \quad 15.46$ $15 \quad 125 \quad 50.04 \quad 19 \quad 20.12 \quad 155 \quad 18.84$

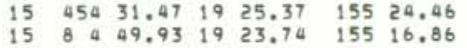
$\begin{array}{lllllll}15 & 2222 & 22.44 & 19 & 4.15 & 156 & 40.51\end{array}$

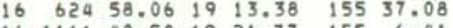
$\begin{array}{lllllll}16 & 1116 & 40.50 & 19 & 21.77 & 155 & 6.41 \\ 16 & 1323 & 58.90 & 19 & 22.63 & 155 & 29.09\end{array}$

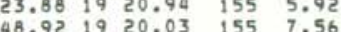
$\begin{array}{rrrrrrr}17 & 756 & 7.87 & 19 & 21.71 & 155 & 25.33 \\ 17 & 1036 & 14.22 & 19 & 20.32 & 155 & 7.19\end{array}$

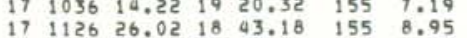

$\begin{array}{lllllll}17 & 1734 & 23.67 & 19 & 20.56 & 155 & 1.01\end{array}$

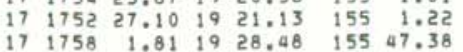

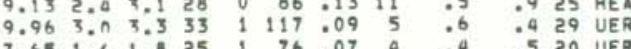

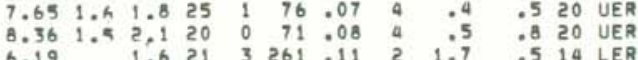
6.93 2. $2.6 .630 \quad 0 \quad 95.12 \quad 6 \quad .5 \quad .928$ UER $\begin{array}{rrrrrrrrrrrr}8.62 & 2.1 & 2.7 & 32 & 0 & 66 & 13 & 4 & .4 & .6 & 24 & \text { UER } \\ .05 & 3.1 & 2.6 & 41 & 8 & 306 & .12 & 85 & 7.2 & 1.8 & 34 & 018\end{array}$ $\begin{array}{llllllllllll}8.51 & 2.0 & 1.9 & 26 & 0 & 47 & .12 & 8 & .4 & 1.1 & 21 & \text { UKF }\end{array}$

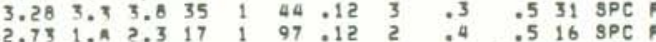

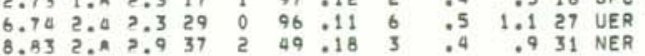
$\begin{array}{llllllllllllll}7.22 & 2.1 & 2.1 & 31 & 0 & 89 & .13 & 6 & .5 & .8 & 30 & \text { UER }\end{array}$

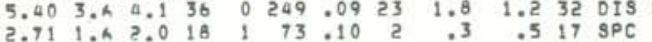

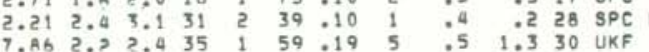
$\begin{array}{lllllllllllll}0.97 & 2.9 & 7.2 & 36 & 1 & 36 & .14 & 6 & .4 & .5 & 32 & \text { UKF }\end{array}$ $6.253 .32 .628 \quad 1140$ is $0 \quad 7$ :9 25 MER

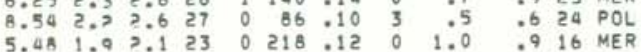
$\begin{array}{lllllllllllll}8.69 & 2.8 & 3.2 & 33 & 1 & 93 & .12 & 2 & .7 & .5 & 28 & \text { MER }\end{array}$

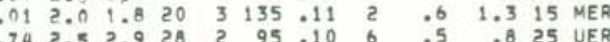

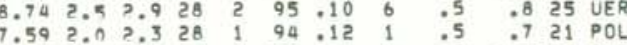

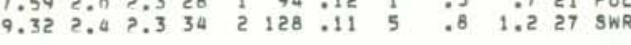
$\begin{array}{lllllllllll}7.50 & 2.3 & 2.3 & 27 & 2 & 117 & .13 & 1 & .5 & .7 & 23 \\ 3 & \text { MER }\end{array}$

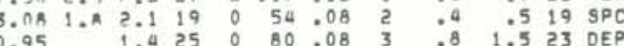

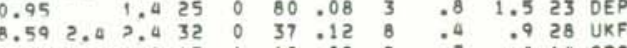
250 .

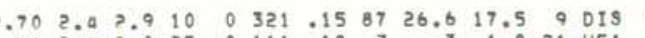

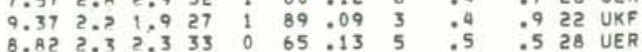
$\begin{array}{llllllllllll}9.79 & 2.4 & 2.7 & 35 & 2 & 100 & .10 & 6 & .4 & .5 & 31 & \text { MER }\end{array}$ $\begin{array}{lllllllllll}0.09 & 2.0 & 2.7 & 37 & 3 & 85 & .13 & 4 & .4 & .4 & 30 \\ 4 & \text { HEA }\end{array}$

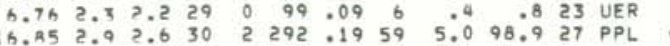

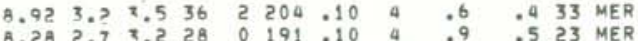

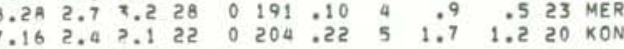

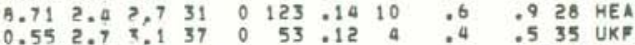

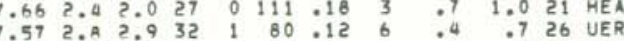
$8.972 .75 .9340097 .0950 .5 \quad .432$ UER

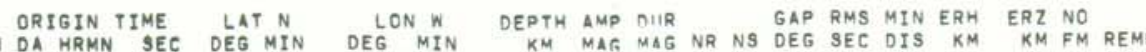

1978 JUN $172116 \quad 7.16 \quad 19 \quad 19.23 \quad 155 \quad 13.58$

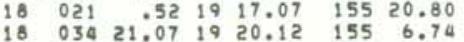

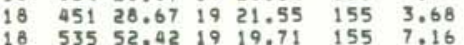
$\begin{array}{lllllll}18 & 855 & 55.59 & 19 & 21.88 & 155 & 4.32\end{array}$ $19.1035 \quad 50.661918 .33 \quad 15516.46$

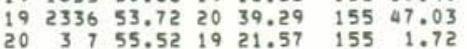

$\begin{array}{lllllll}20 & 312 & 48.70 & 19 & 25.53 & 155 & 16.49\end{array}$ $20 \quad 814 \quad 56.40 \quad 19 \quad 19.76 \quad 155 \quad 8.21$ $020 \quad 44.12 \quad 19 \quad 19.45 \quad 155 \quad 13.35$ $\begin{array}{lllllll}21 & 135 & 52.16 & 19 & 16.95 & 155 & 22.56 \\ 21 & 611 & 32.78 & 19 & 20.39 & 155 & 13.23\end{array}$ $\begin{array}{lllllll}21 & 1847 \quad 35.06 & 19 & 16.09 \quad 156 & 10.69\end{array}$ $21 \quad 1919 \quad 54.1719 \quad 13.15 \quad 156 \quad 20.48$

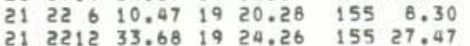
$\begin{array}{lllllll}22 & 013 & 48.74 & 19 & 19.95 & 155 & 6.83\end{array}$ $\begin{array}{lllllll}22 & 013 & 48.74 & 19 & 19.95 & 155 & 6.83 \\ 22 & 137 & 4.43 & 19 & 20.49 & 155 & 6.91\end{array}$

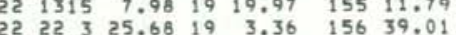

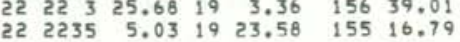

$23 \quad 147 \quad 58.56 \quad 19 \quad 19.16 \quad 155 \quad 15.46$

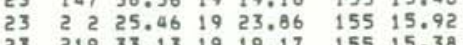

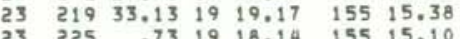
$\begin{array}{lllllll}23 & 225 & .73 & 19 & 18.14 & 155 & 15.10 \\ 23 & 519 & 57.26 & 19 & 23.27 & 155 & 15.13\end{array}$

$\begin{array}{llllllll}23 & 621 & 3.31 & 19 & 23.48 & 155 & 15.24\end{array}$ $\begin{array}{lllllll}23 & 2341 & 17.57 & 19 & 18.19 & 155 & 15.37\end{array}$ $\begin{array}{lllllll}24 & 114 & 4.30 & 18 & 45.60 & 155 & 14.31 \\ 24 & 131 & 14.27 & 19 & 19.32 & 155 & 11.90\end{array}$

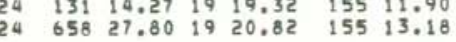

$\begin{array}{lllllllll}24 & 9 & 7 & 27.78 & 19 & 20.03 & 155 & 15.63\end{array}$

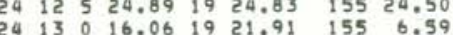
$241940 \quad 38.5919 \quad 35.56 \quad 15526.11$

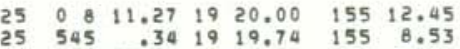
$25 \quad 931 \quad 15.67 \quad 19 \quad 19.19 \quad 155 \quad 9.04$ $25 \quad 1536 \quad 35.75$ 19 27.23 i54 51.96 $25 \quad 1740 \quad 39.15 \quad 19 \quad 18.55 \quad 155 \quad 15.24$ $\begin{array}{lllllll}25 & 2039 & 59.70 & 19 & 23.26 & 155 & 16.84 \\ 25 & 2134 & 45.40 & 19 & 20.43 & 155 & 3.65\end{array}$

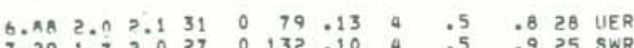

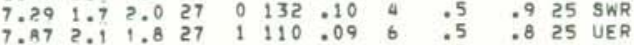
$\begin{array}{llllllllllll}7.39 & 2.1 & 2.1 & 29 & 1 & 103 & 14 & 3 & .5 & .8 & 28 & \text { MER } \\ 7.29 & 2.0 & 2.1 & 29 & 1 & 112 & .13 & 5 & .5 & 1.0 & 27 & \end{array}$ \begin{tabular}{lllllllllll}
6.16 & 2.3 & 2.6 & 26 & 1 & 88 & .12 & 4 & .5 & .9 & 18 \\
\hline
\end{tabular}

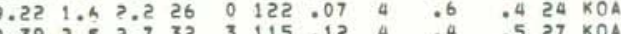

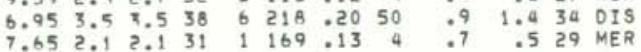
$\begin{array}{llllllllllllll}2.58 & 2.0 & 2.9 & 22 & 1 & 46 & .12 & 1 & .4 & .3 & 18 & \text { SPC F }\end{array}$

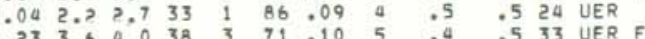

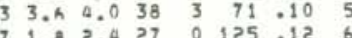

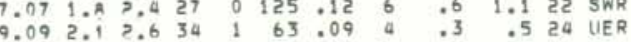
$9.97 \quad 2.011 \quad 0 \quad 316.12 \quad 32 \quad 19.1 \quad 9.511$ 015: $\begin{array}{lllllllllllll}0.06 & 2.9 & 7.8 & 13 & 0 & 327 & .08 & 50 & 24.7 & 7.7 & 13 & 019 & 019\end{array}$

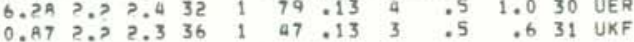

$\begin{array}{llllllllllllll}8.56 & 2.7 & 7.1 & 32 & 0 & 113 & .10 & 5 & .6 & .5 & 30 & \text { UER }\end{array}$

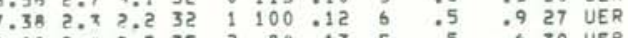

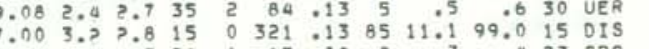

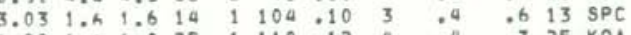

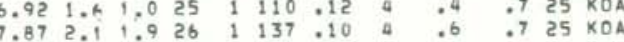

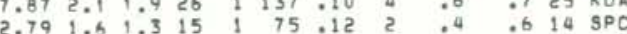

$\begin{array}{llllllllllll}3.2 A & 2.1 & 0.0 & 23 & 1 & 83 & 010 & 2 & .4 & .5 & 22 & 3 P C\end{array}$

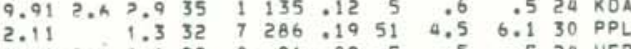

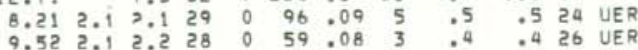

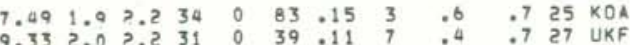

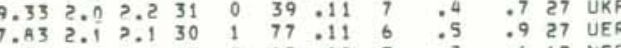
$\begin{array}{rlllllllllll}.93 & 1.6 & .7 & 23 & 4 & 69 & 18 & 5 & .3 & .6 & 17 & \text { NER } \\ 7.76 & 7.4 & 1.8 & 28 & 0 & 63 & .13 & 3 & .5 & .7 & 23 & \text { UER }\end{array}$

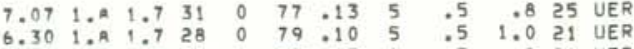

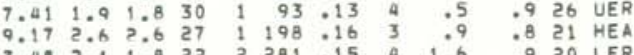
$9.402 .1 \quad 2.431 \quad 1129.09 \quad 4 \quad .5 \quad .426 \quad K O A$

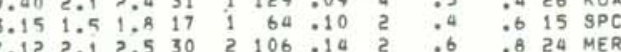


ORIGIN TIME LAT NGP RMS MIN ERH ERZ NO TEAR MON DRIGIN TIME LARM SEC OEG MIN DEG MIN DERTM AMP RIIR NR NG GAP RMS MIN ERH ERZ NO REMT

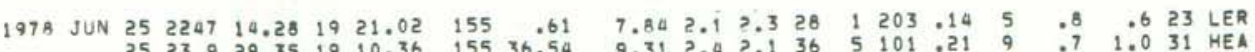

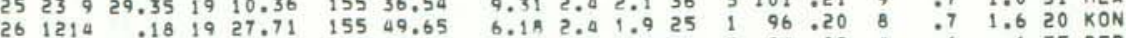

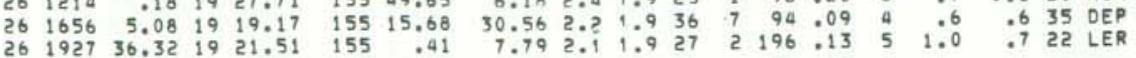

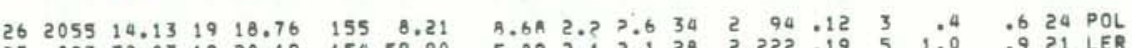

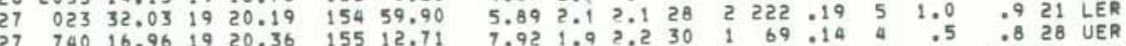

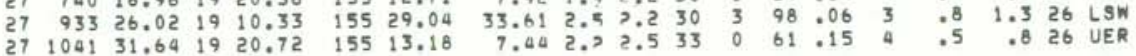

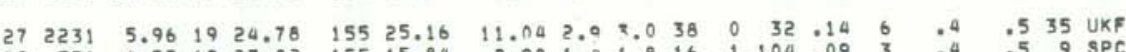
$\begin{array}{llllllllllll} & & 5 & 5 & \text { SPC }\end{array}$

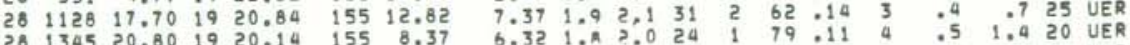

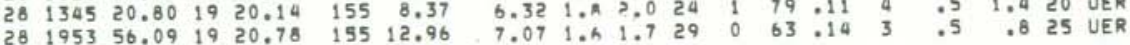

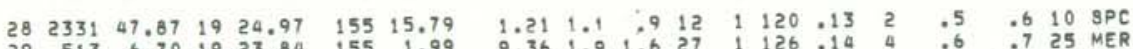

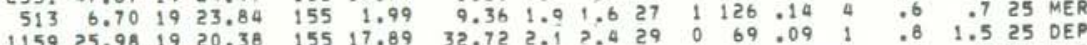

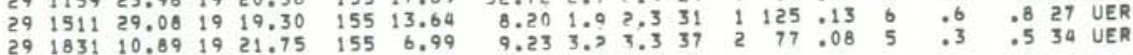

$29204 \quad 9.50 \quad 1925.50 \quad 155 \quad 24.31$ (9.3 $155 \quad 7.60$ $30 \quad 146 \quad 11.051920 .14 \quad 155 \quad 6.25$

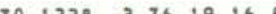
$\begin{array}{lllllll}30 & 1420 & 34.92 & 19 & 19.63 & 155 & 10.35\end{array}$

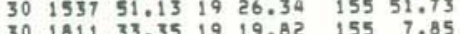

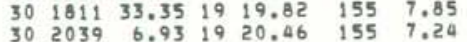

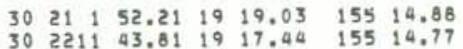
$30223122.1019 \quad 18.35 \quad 15515.48$

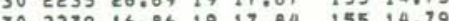
\begin{tabular}{lllllllllllllllllll}
30 & 2246 & 4.68 & 19 & 20.45 & 155 & 17.80 & 31.55 & 2.3 & 2.4 & 36 & 2 & 68 & .10 & 1 & .7 & .932 & 32 & OEP \\
30 & 2248 & 37.23 & 19 & 19.31 & 155 & 12.04 & 3.15 & 2.2 & 2.3 & 33 & 3 & 95 & .13 & 5 & .5 & .724 & 24 & $0 R$ \\
\hline
\end{tabular} JUL $14924.151920 .25 \quad 155 \quad 15.61$ $45140.91 \quad 19 \quad 14.19 \quad 156 \quad 14.61$ $\begin{array}{lllllll}1 & 653 & 28.46 & 19 & 20.21 & 155 & 2.24 \\ 1 & 917 & 58.44 & 19 & 19.68 & 155 & 7.09 \\ 1 & 918 & 13.27 & 19 & 18.47 & 155 & 6.73\end{array}$ $\begin{array}{llllll}918 & 13.27 & 19 & 18.47 & 155 & 6.73\end{array}$

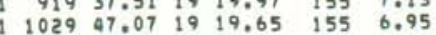
$\begin{array}{lllllll}1 & 1258 & 35.88 & 19 & 18.36 & 155 & 14.48 \\ 1 & 1541 & 25.84 & 19 & 19.73 & 155 & 7.25\end{array}$ $1548 \quad 6.56 \quad 19 \quad 16.98 \quad 155 \quad 30.75$

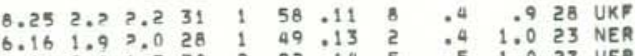

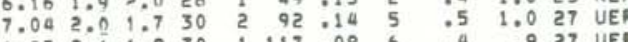
$\begin{array}{rrrrrrrrrrrr}6.87 & 2.1 & 1.9 & 30 & 1 & 117 & .09 & 6 & .4 & .9 & 27 & \text { UER } \\ 8.07 & 2.1 & 2.2 & 31 & 1 & 81 & .14 & 4 & .5 & .8 & 27 & \text { POL }\end{array}$

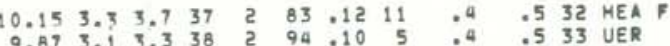

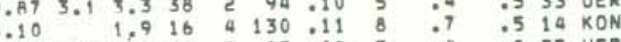

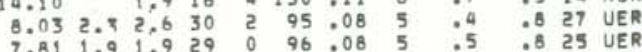
$\begin{array}{lllllllllllll}7.43 & 1 . A & 1.8 & 30 & 0 & 88 & -12 & 4 & .5 & .8 & 24 & \text { UER }\end{array}$ 7.90

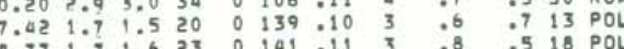

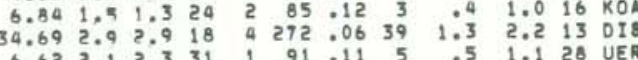

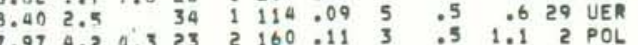

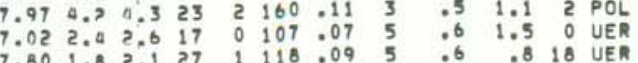
$\begin{array}{llllllllllll}7.02 & 1 . \wedge & 1.8 & 25 & 1 & 118 & .09 & 3 & .5 & .7 & 20 & \text { POL }\end{array}$

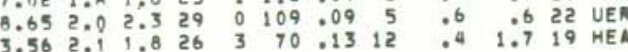

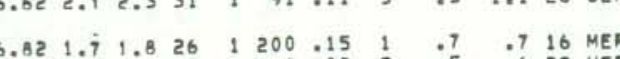

HVO EARTHQUAKE SUMMARY LIST

PAGE 24

ORIGIN TIME
LAT N

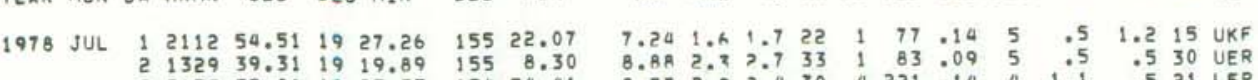
$\begin{array}{lllllll}2 & 1329 & 39.31 & 19 & 19.89 & 155 & 8.30\end{array}$ $22133 \quad 57.86 \quad 1925.55 \quad 15454.41$ $3 \quad 1660.27 \quad 1920.66 \quad 155 \quad 6.70$

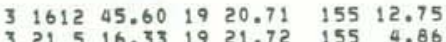

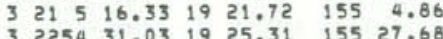

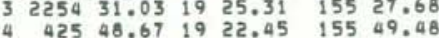
$4 \quad 61815.091925 .60 \quad 15524.90$

$\begin{array}{lllllll}4 & 634 & 27.59 & 19 & 21.30 & 155 & 15.08\end{array}$ $4 \quad 738 \quad 2.79 \quad 19 \quad 20.87 \quad 155 \quad 10.99$

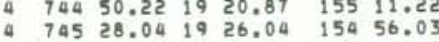

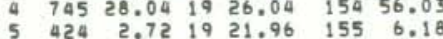

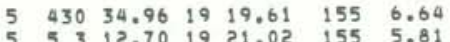
$\begin{array}{rrrrrrr}5 & 53 & 32.70 & 19 & 21.02 & 155 & 5.81 \\ 5 & 1218 & 52.21 & 19 & 33.91 & 155 & 38.21\end{array}$

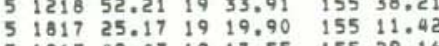
$51913 \quad 49.43 \quad 1917.55 \quad 15529.16$

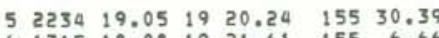

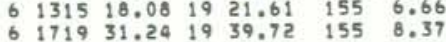

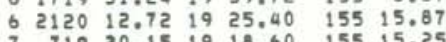

$\begin{array}{llllll}1131 & 29.20 & 19 & 12.09 & 156 & 7.13\end{array}$ $\begin{array}{lllllll}7 & 1910 & 6.41 & 19 & 19.55 & 155 & 8.53\end{array}$

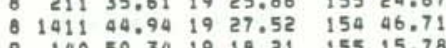
$75438.19 \quad 19 \quad 21.11 \quad 155 \quad 13.17$ $845 \quad 31.42 \quad 19 \quad 19.15 \quad 155 \quad 11.2$

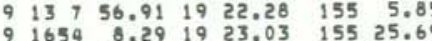
$91837 \quad 37.90 \quad 19 \quad 14.86 \quad 155 \quad 20.54$ $9 \begin{aligned} & 1841 \quad 32.22 \quad 1920.39 \quad 155 \quad 3.58 \\ & 9\end{aligned}$ $0.194324 .541914 .46 \quad 15520.36$

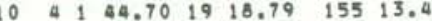
$10 \quad 1026 \quad 24.86 \quad 19 \quad 18.65 \quad 15513.22$ $\begin{array}{lllllll}10 & 1322 & 5.77 & 19 & 11.47 & 155 & 30.72\end{array}$ $\begin{array}{llllll}1621 & 40.76 & 19 & 21.94 & 155 & 3.76\end{array}$ $11 \quad 347 \quad 46.06 \quad 19$ $11 \quad 114 \quad 18.73 \quad 19 \quad 30.19 \quad 155 \quad 35.29$ $\begin{array}{lllllll}12 & 330 & 2.84 & 19 & 20.08 & 155 & 6.46\end{array}$

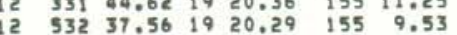

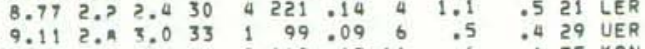
$9.352 .9 \$ 138 \quad 2 \quad 162.12 \quad 11 \quad .6 \quad .635$ KON $\begin{array}{llllllllllll}7.84 & 1.2 & 1.3 & 25 & 0 & 65 & 12 & 4 & .5 & .7 & 17 & \text { UER } \\ 9.07 & 3.4 & 3.6 & 37 & 0 & 80 & : 11 & 5 & : 6 & .4 & 36 & \text { MER F }\end{array}$ $11.083 .0 \quad 3.5 \quad 37 \quad 1 \quad 38.11 \quad 5 \quad .9 \quad .532$ UKF

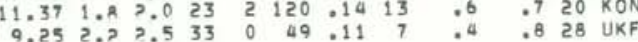
$\begin{array}{lllllllllllll}7.71 & 1.9 & 1.8 & 26 & 1 & 66 & .10 & 3 & .5 & .8 & 23 & \text { KOA } \\ 8.41 & 1: 9 & 1.7 & 27 & 1 & 72 & : 11 & 3 & .5 & .7 & 20 & \text { UER }\end{array}$

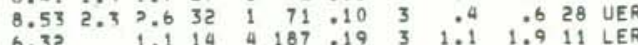

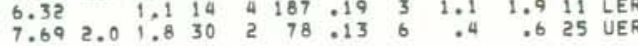
$\begin{array}{llllllllllll}7.43 & 2.1 & 7.4 & 37 & 1 & 125 & 012 & 5 & .5 & .5 & 31 & \text { UER }\end{array}$

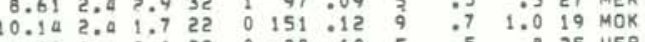

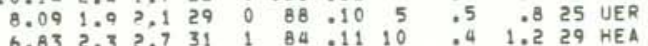
$\begin{array}{rllllllllll}7.04 & 1.9 & 1.7 & 27 & 0 & 66 & .12 & 6 & .5 & 1.4 & 25 \\ 0.5 & \text { HEA }\end{array}$

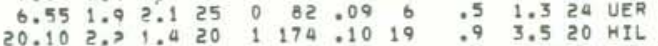

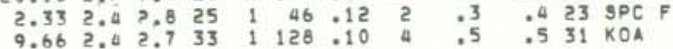
$\begin{array}{lllllllllllll}26.40 & 3.1 & 7.9 & 12 & 0 & 322 & .12 & 27 & 19.5 & 7.7 & 12 & 01 \mathrm{~S}\end{array}$

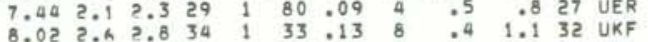

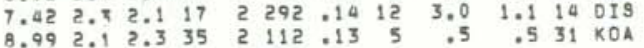
$\begin{array}{lllllllllllll}7.07 & 1.9 & 2.1 & 30 & 1 & 57 & .16 & 3 & .5 & .8 & 29 \text { UER }\end{array}$

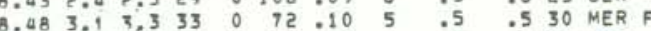

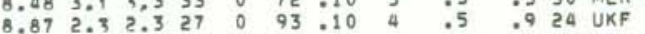
$\begin{array}{lllllllllllll}8.68 & 1.02 & 2.0 & 32 & 0 & 156 & .11 & 7 & .6 & .6 & 24 & \mathrm{HLP}\end{array}$

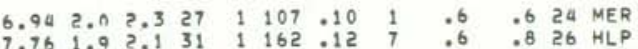

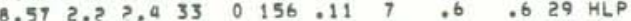

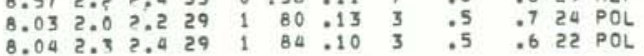
$\begin{array}{llllllllllll}7.43 & 2.2 & 0.1 & 27 & 2 & 90 & .16 & 6 & .7 & 1.2 & 20 & \mathrm{LSW}\end{array}$

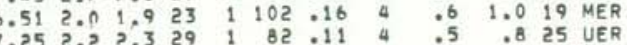

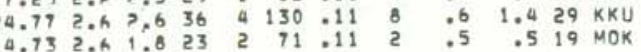
$\begin{array}{rrrrrrrrrrrr}.81 & 2.1 & 7.2 & 29 & 1 & 116 & 110 & 6 & .6 & .7 & 25 & \text { UER } \\ 33 & 1.7 & 1.7 & 28 & 0 & 80 & : 13 & 4 & .5 & .8 & 22 & \text { UER }\end{array}$

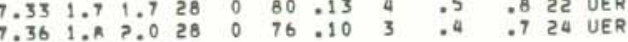




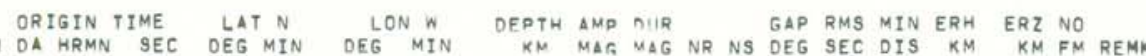

1978 JUL $12 \quad 1133 \quad 10.16 \quad 19 \quad 17.98 \quad 155 \quad 11.45$ $\begin{array}{lllllll}12 & 1230 & 1.91 & 19 & 21.25 & 155 & 5.95 \\ 12 & 2126 & 10.78 & 19 & 19.32 & 155 & 7.34\end{array}$

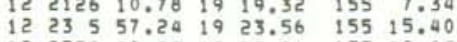
$12 \quad 233222.22 \quad 1923.94 \quad 15528.83$ $\begin{array}{llllllll}13 & 529 & 9.14 & 19 & 19.81 & 155 & 10.57 \\ 13 & 8 & 3 & 48.97 & 19 & 13.30 & 156 & 14.48\end{array}$ $13134922.63 \quad 1921.98$ 155 4.33

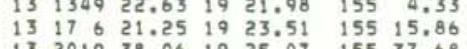

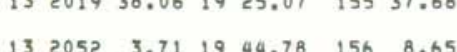
$\begin{array}{rrrrrrr}13 & 2052 & 3.71 & 19 & 44.78 & 156 & 8.65 \\ 13 & 2129 & 51.47 & 19 & 13.66 & 155 & 28.58\end{array}$ $\begin{array}{lllll}13 & 22 & 1 & 15.70 \\ 14 & 256 & 37.19\end{array}$

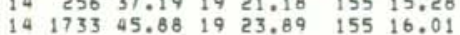
$14 \quad 1849 \quad 28.99 \quad 19 \quad 19.92 \quad 155 \quad 8.05$ $\begin{array}{lllllll}14 & 1938 & 39.96 & 19 & 20.84 & 155 & 6.46\end{array}$ 14 $2250 \quad 9.391917 .44 \quad 15512.93$

$\begin{array}{lllllll}15 & 645 & 43.66 \quad 19 & 13.69 & 155 & 25.79\end{array}$

$\begin{array}{r}1511954.751917 .80 \\ +15113847.151920 .20 \quad 15513.57 \\ \hline 15\end{array}$ $\begin{array}{rrrrrrr}15 & 1914 & 58.30 & 19 & 19.67 & 155 & 11.67 \\ 16 & 829 & 41.78 & 19 & 21.05 & 155 & 1.02\end{array}$

$\begin{array}{llllllll}16 & 1443 & 47.73 & 19 & 22.80 & 155 & 20.70\end{array}$ $\begin{array}{lllllll}16 & 1644 & 33.76 & 19 & 20.75 & 155 & 5.68\end{array}$

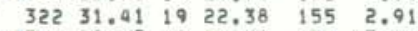

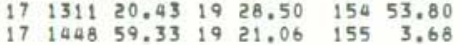
$\begin{array}{lllllll}17 & 1525 & 40.84 & 19 & 22.36 & 155 & 6.09\end{array}$ 1833134.971920 .10015511 .86

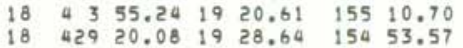

$\begin{array}{lllllll}18 & 1421 & 58.69 & 19 & 19.81 & 155 & 11.13\end{array}$

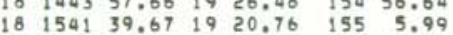
$\begin{array}{rrrrrrr}19 & 1359 & .49 & 19 & 21.09 & 155 & 7.17 \\ 20 & 018 & 5.75 & 19 & 20.84 & 155 & 12.97\end{array}$

$\begin{array}{llllllll}20 & 2 & 6 & 1.13 & 19 & 20.35 & 155 & 11.61\end{array}$

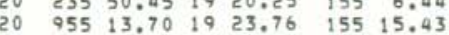
$\begin{array}{lllllll}21 & 120 & 7.34 & 19 & 23.00 & 155 & 16.93 \\ 21 & 239 & 21.69 & 19 & 19.92 & 155 & 13.00\end{array}$

$\begin{array}{lllllll}21 & 338 & 15.01 & 19 & 19.24 & 155 & 8.46\end{array}$

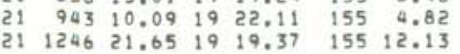

$\begin{array}{llllllllllll}8.45 & 2.1 & 2.1 & 29 & 2 & 168 & .17 & 4 & .6 & .7 & 21 & \text { POL } \\ 7.77 & 2.7 & 2.3 & 29 & 1 & 146 & .12 & 7 & .6 & .7 & 28 & M E R \\ 5.72 & 2.0 & 0.1 & 28 & 1 & 116 & .12 & 4 & .5 & 1.5 & 26 & \text { UER }\end{array}$ $\begin{array}{llllllllllll}5.22 & 2.0 & 0.1 & 28 & 1 & 116 & -12 & 4 & .5 & 1.5 & 26 & \text { UER } \\ 3.06 & 1.4 & 1.8 & 17 & 1 & 51 & .11 & 2 & : 5 & .6 & 16 & \text { SPC }\end{array}$

$9.222 .22 .129 \quad 0 \quad 48$

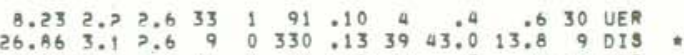

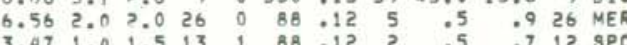
$\begin{array}{llllllllllll}3.47 & 1.9 & 1.5 & 13 & 1 & 88 & .12 & 2 & .5 & .7 & 12 & \mathrm{SPC} \\ .00 & 2.5 & 7.1 & 11 & 0 & 75 & .25 & 5 & .9 & 3.8 & 11 & \text { MOK }\end{array}$

$16.08 \quad 2.92 .3 \quad 30 \quad 0 \quad 288 \quad .16 \quad 33 \quad 4.999 .0 \quad 28$ KON

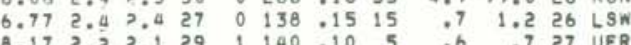

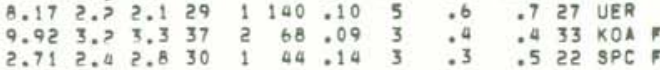

$\begin{array}{llllllllllll}5.36 & 1.9 & 2.0 & 23 & 0 & 88 & .09 & 5 & .5 & 1.2 & 17 & \text { UER } \\ 6.72 & 2.1 & 2.1 & 30 & 1 & 98 & .08 & 6 & .5 & .9 & 25 & \text { UER }\end{array}$

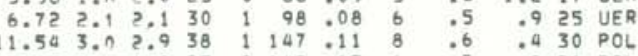

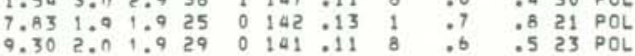

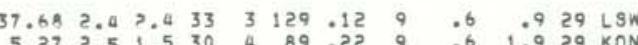

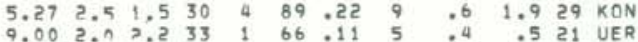

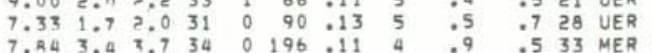

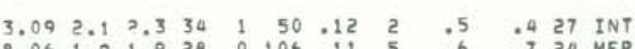

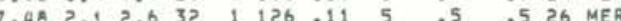

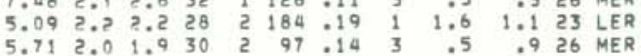

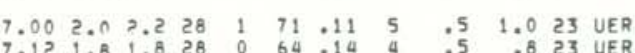
$\begin{array}{llllllllllllll}7.53 & 1.9 & 0.1 & 30 & 1 & 81 & .13 & 5 & .5 & .7 & 26 & \text { UER }\end{array}$

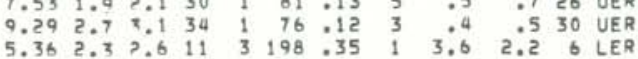
\begin{tabular}{lllllllllll}
8.05 & 1.7 & 1.9 & 28 & 0 & 90 & .09 & 5 & .4 & .6 & 27 \\
\hline
\end{tabular}

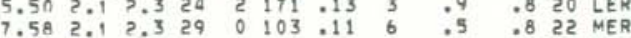

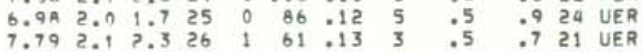
$\begin{array}{llllllllllll}7.00 & 2.0 & 1.6 & 25 & 0 & 78 & .10 & 4 & .5 & .8 & 21 & \text { UER }\end{array}$

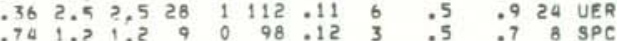

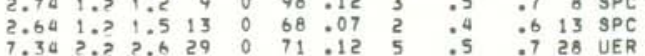
$\begin{array}{llllllllllll}7.77 & 2.2 & 0.1 & 28 & 0 & 83 & -11 & 4 & .6 & .7 & 27 & \text { UER }\end{array}$

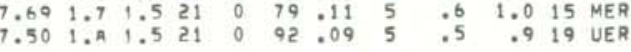

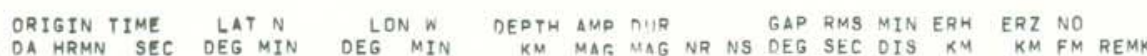

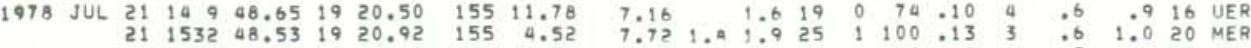

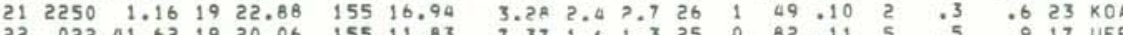

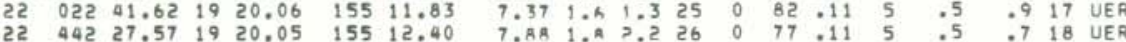

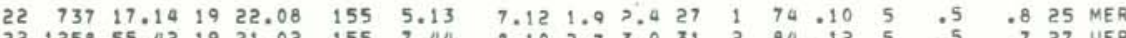

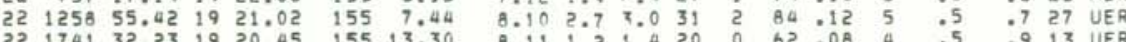

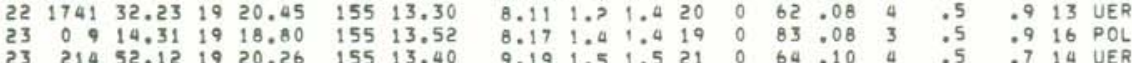

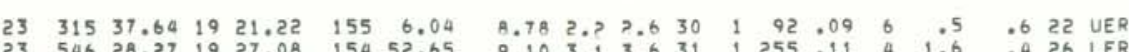

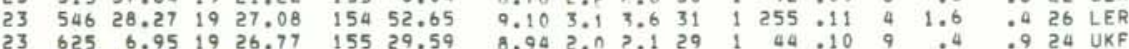

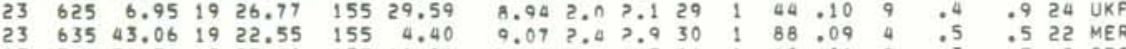

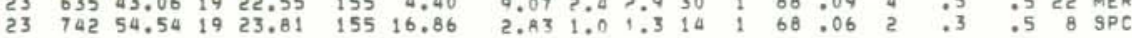

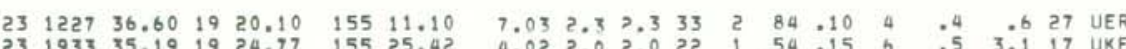

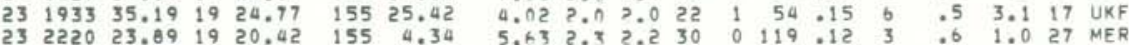

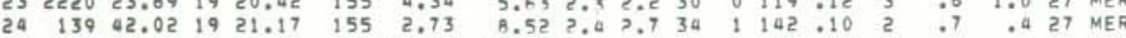

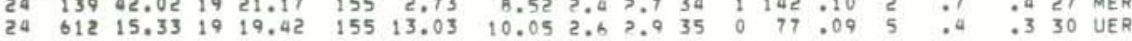
\begin{tabular}{llllllllllllllllll}
24 & 810 & 35.11 & 19 & 19.14 & 155 & 8.02 & $9.1 n$ & 2.1 & 2.1 & 16 & 1 & 98 & .08 & 3 & .8 & .7 & 10 \\
\hline & UER
\end{tabular}

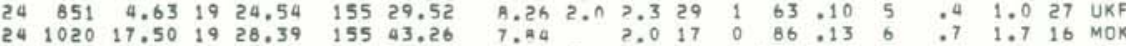

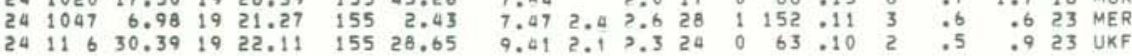
$\begin{array}{lllllllllllllllllll}24 & 1136 & 19.00 & 19 & 19.88 & 155 & 8.05 & 7 . A 9 & 2.7 & 2.6 & 27 & 1 & 88 & 0.10 & 5 & .5 & .925 & 25 & \text { UER }\end{array}$

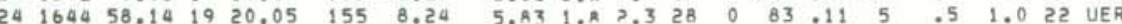

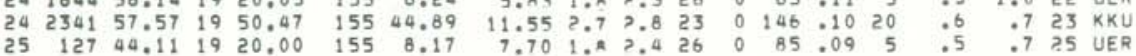

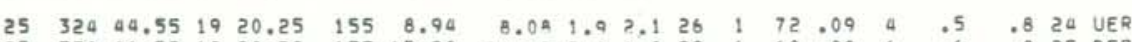

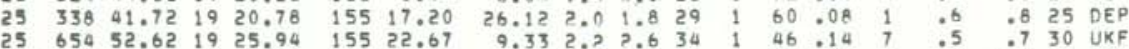

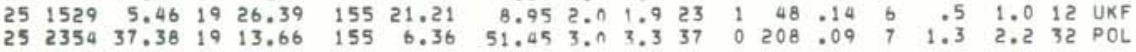
$\begin{array}{llllllllllllllllllll}26 & 1027 & 8.98 & 19 & 30.14 & 155 & 16.99 & 13.90 & 2.4 & 2.9 & 33 & 2 & 48 & .10 & 9 & .3 & .4 & 30 & 6 L N\end{array}$

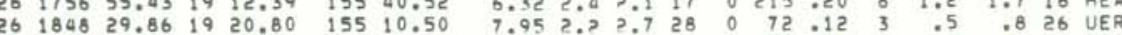

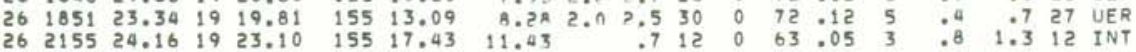

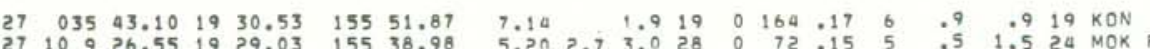

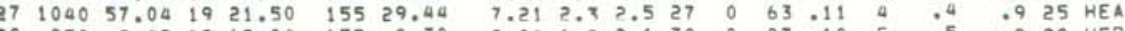

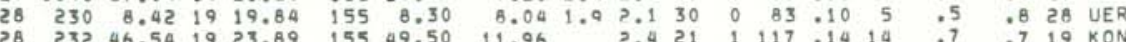

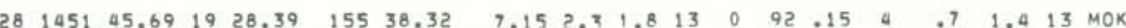

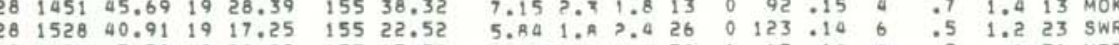

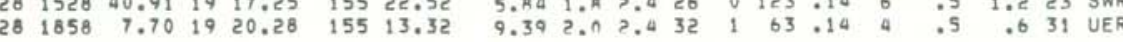


ORIGIN TIME LATN LON W DEPTH AMP RIR N GAP RMS MIN ERH ERZ NO

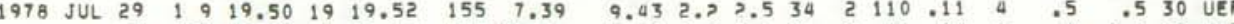
$29 \quad 427 \quad 49.601927 .05 \quad 155 \quad 21.61$

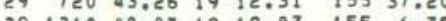
$291210 \quad 48.07 \quad 1919.83 \quad 155 \quad 6.35$

$29 \begin{array}{llllll}1648 & 52.18 & 19 & 23.90 & 155 & 15.55\end{array}$ $\begin{array}{lllllll}29 & 19 & 20.90 & 19 & 22.86 & 155 & 25.13 \\ 29 & 1953 & 4.33 & 19 & 23.89 & 155 & 17.56\end{array}$

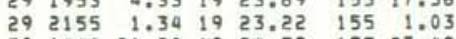

$30.140921 .901924 .32 \quad 155$ 27. 03

$\begin{array}{lllllll}31 & 1626 & 11.11 & 19 & 24.16 & 155 & 27.51 \\ 31 & 1631 & 13.29 & 19 & 17.70 & 155 & 12.97\end{array}$ $31 \quad 185853.841924 .20 \quad 15527.59$ 31 2317 49.33 i9 23.80 155 15.73

AUG $\begin{array}{rrrrrrr}1 & 028 & 5.62 & 19 & 24.38 & 155 & 17.07 \\ 1 & 435 & 15.76 & 19 & 27.17 & 155 & 22.09\end{array}$

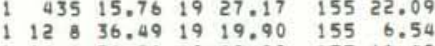

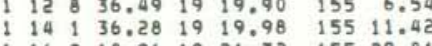

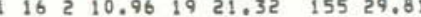
$\begin{array}{lllllll}1 & 1611 & 13.30 & 19 & 55.59 & 155 & 27.32 \\ 1 & 198 & 15.33 & 19 & 20.61 & 155 & 11.23\end{array}$

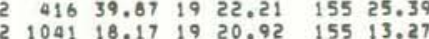

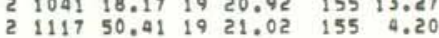
$21640 \quad 55.72 \quad 19 \quad 23.60 \quad 155 \quad 16.66$ 2
3 $325123.44 \quad 1921.92 \quad 155 \quad 6.55$

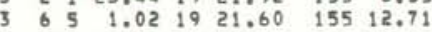

$\begin{array}{lllllll}3 & 946 & 59.14 & 19 & 23.78 & 155 & 15.90\end{array}$ 737
4 $\quad 37.8219 \quad 19.89 \quad 155 \quad 8.07$

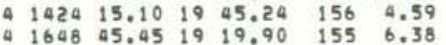
q $1954 \quad 15.74 \quad 19 \quad 24.29 \quad 155 \quad 29.87$ $5428 \quad 55.61 \quad 1923.45 \quad 155 \quad 15.10$

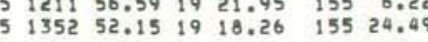

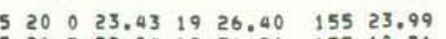

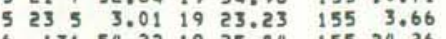
$\begin{array}{llllll}6 & 131 & 54.22 & 1925.84 & 155 & 24.26 \\ 6 & 517 & 33.02 & 1922.00 & 155 & 5.55\end{array}$ $6 \quad 1639$
7 $7 \quad 64 \quad 1.681921 .15 \quad 155 \quad 2.60$

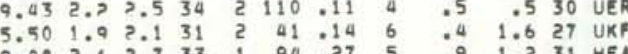

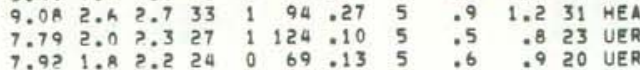
$\begin{array}{rrrrrrrrrrrr}2.85 & 1.2 & 1.3 & 9 & 1 & 107 & 11 & 3 & .6 & .9 & 8 & \text { SPC } \\ 0.59 & 2.0 & 2.2 & 27 & 0 & 89 & .09 & 4 & .4 & .6 & 26 & \text { UKF }\end{array}$ $\begin{array}{lllllllllllll}5.21 & 1.3 & 1.2 & 12 & 1 & 59 & .15 & 2 & .6 & 1.1 & 11 & \mathrm{sP}\end{array}$

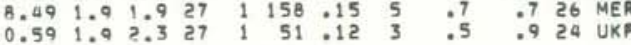

$\begin{array}{lllllllllllll}10.58 & 2.2 & 2.4 & 25 & 0 & 48 & 10 & 3 & .5 & .8 & 20 & \text { UKF }\end{array}$

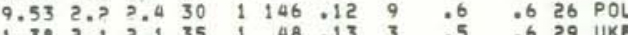
$0.082 .1 ? .429 \quad 2 \quad 138.10 \quad 5 \quad .6 \quad .5$ 24 MER $\begin{array}{lllllllllllll}1.59 & 1.2 & 1.4 & 11 & 0 & 58 & .04 & 1 & .3 & .4 & 11 & \mathrm{SPC}\end{array}$

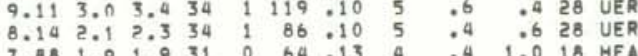
$\begin{array}{llllllllllll}14.24 & 2.4 & 1.9 & 31 & 2 & 175 & .17 & 36 & 1.4 & 4.5 & 26 & \mathrm{KKU}\end{array}$

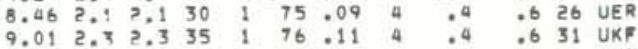

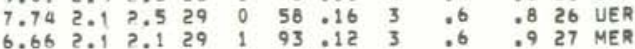

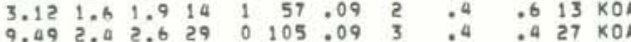

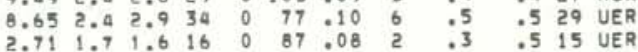
$\begin{array}{lllllllllllll}2.65 & 2.0 & 2,3 & 19 & 1 & 78 & .11 & 3 & .4 & .5 & 18 & \mathrm{SPC}\end{array}$

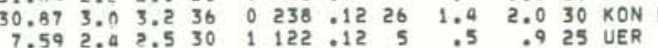

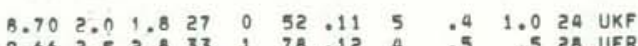

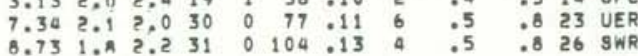
$\begin{array}{lllllllllllll}0.10 & 3.0 & 3.2 & 35 & 0 & 42 & .13 & 6 & .5 & .7 & 34 & \text { UKF }\end{array}$ $\begin{array}{llllllllllllll}1.24 & 2.1 & 2.4 & 30 & 0 & 136 & .11 & 15 & .5 & .8 & 21 & \text { HIL }\end{array}$

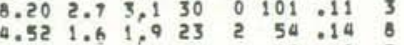
$8.152 .0 ? .430 \quad 0 \quad 77.1250 \quad .5 \quad .723$ MER $\begin{array}{llllllllllll}9.15 & 1.9 & 2.3 & 29 & 0 & 69 & 11 & 4 & .4 & .5 & 22 & \text { UER } \\ 6.57 & 2.0 & 2.1 & 30 & 0 & 49 & .15 & 6 & .4 & 1.5 & 28 & \text { UKF }\end{array}$

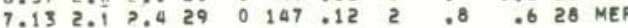

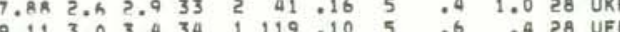
$\begin{array}{llllllllllllll}2.93 & 2.1 & 2.5 & 20 & 1 & 70 & .09 & 3 & .3 & .5 & 19 & \mathrm{sPC}\end{array}$

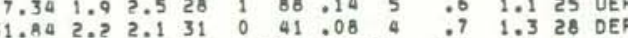

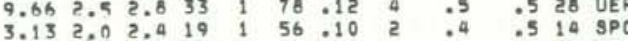

ORIGIN TIME LATN LON W 1978 AUG $? 1256 \quad 30.33 \quad 19 \quad 16.76 \quad 155 \quad 21.59$ $\begin{array}{lllllll}7 & 1435 & 37.83 & 19 & 20.42 & 155 & 13.05 \\ 7 & 22 & 71.11 & 19 & 20.72 & 155 & 10.37\end{array}$

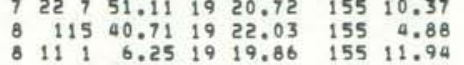

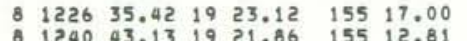
$\begin{array}{lllllll}8 & 1240 & 43.13 & 19 & 21.86 & 155 & 12.81 \\ 8 & 2110 & 10.28 & 19 & 18.19 & 155 & 13.18\end{array}$

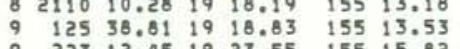

$9443 \quad 22.04 \quad 1927.30 \quad 154 \quad 49.4$ $9122549.02 \quad 1918.37 \quad 15523.75$

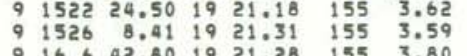
$9337 \quad 20.91 \quad 19 \quad 23.82 \quad 155 \quad 16.10$ $\begin{array}{rrrrrrr}9 & 2337 & 24.91 & 19 & 23.82 & 155 & 16.110 \\ 10 & 132 & 40.92 & 19 & 30.99 & 155 & 23.95\end{array}$

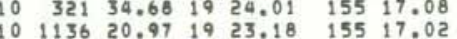
$10 \quad 1216 \quad 18.34 \quad 1926.52 \quad 154 \quad 53.37$ $\begin{array}{lllllll}10 & 1327 & 1.75 & 19 & 24.78 & 155 & 23.59\end{array}$ $\begin{array}{rrrrrrr}10 & 1444 & 21.86 & 19 & 19.77 & 155 & 13.34 \\ 11 & 414 & 34.67 & 19 & 23.64 & 155 & 15.28\end{array}$ 11
11 92139.06 19 22.82 15516.84 $\begin{array}{lllllll}11 & 1054 & 37.00 & 19 & 22.41 & 155 & 29.30\end{array}$ 11 1444 58.70 19 $41.03 \quad 155 \quad 3.15$ $\begin{array}{lllllll}11 & 158 & 51.86 & 20 & 45.36 & 155 & 59.54 \\ 11 & 2316 & 20.48 & 19 & 23.35 & 155 & 26.55\end{array}$ $\begin{array}{lllllll}12 & 052 & 50.53 \quad 19 & 20.05 \quad 155 \quad 6.84\end{array}$

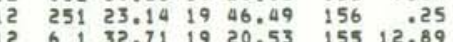
$12 \quad 94942.751951 .50 \quad 155 \quad 52.24$ $\begin{array}{lllllll}12 & 14 & 10.07 & 19 & 18.62 & 155 & 13.53\end{array}$

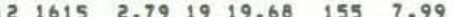

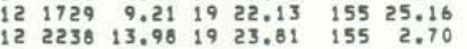

$13 \quad 531 \quad 17.46 \quad 1924.98 \quad 155 \quad 24.48$

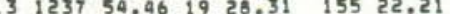
$13201939.18 \quad 1923.05 \quad 15516.95$ $132212 \quad 28.96 \quad 1956.00 \quad 15521.66$

$\begin{array}{lllllll}13 & 2245 & 29.27 & 19 & 20.86 & 155 & 7.33\end{array}$

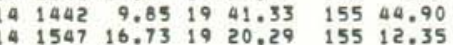

DEPTH AMP NIIR NR GAP RMS MIN ERH ERZ NO
KM MAG MAG NR NS DEG SEC OIS KM KM FM REMK

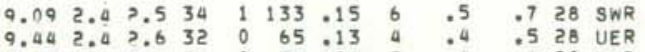

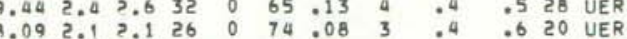

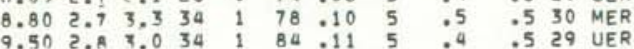

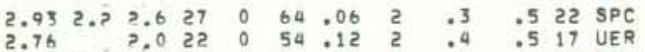
$.443 .74 .036 \quad 0 \quad 94.09 \quad 2 \quad .6 \quad .533$ POL F $\begin{array}{llllllllllll}7.01 & 2.1 & 2.1 & 34 & 1 & 83 & .12 & 3 & .5 & .7 & 24 & P O L \\ 3.09 & 2.1 & 22 & 0 & 43 & 0.12 & 2 & .4 & .6 & 18 & \text { SPC }\end{array}$

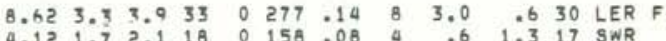

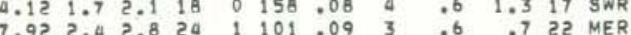

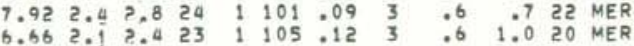
$6.702 .07 .424 \quad 0 \quad 97.13 \quad 3 \quad .7 \quad .821$ MER

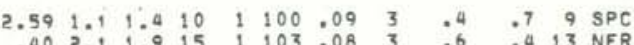

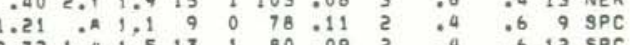

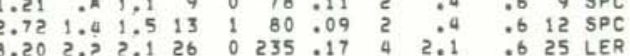
$\begin{array}{lllllllllllll}.20 & 2.1 & 2.4 & 27 & 0 & 57 & .10 & 7 & .4 & .9 & 27 & \text { UKF }\end{array}$

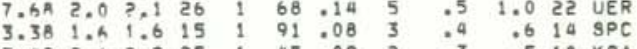
$\begin{array}{rrrrrrrrrrrr}3.42 & 2.1 & 2.9 & 25 & 1 & 47 & .09 & 2 & .3 & .5 & 18 & \text { KOA } \\ 6.58 & 1.7 & 2.3 & 19 & 2 & 205 & .12 & 2 & 1.2 & .7 & 16 & \text { LER }\end{array}$

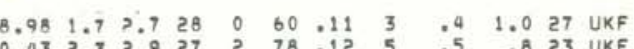

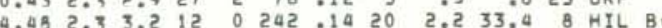

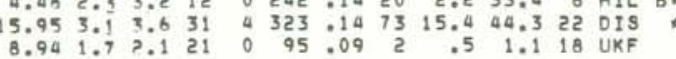

$9.883 .23 .936 \quad 2 \quad 110.09 \quad 5 \quad .5 \quad .432$ UER F

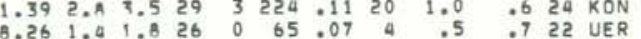
$\begin{array}{rrrrrrrrrrrr}7.35 & 1.2 & 1.3 & 22 & 0 & 66 & .09 & 9 & .5 & .8 & 14 & \text { UER } \\ 3.67 & 1.3 & 2.6 & 24 & 5 & 190 & .09 & 19 & .7 & 1.1 & 23 & \text { KON }\end{array}$ $9.312 .4 \quad 3.132 \quad 2 \quad 84.10 \quad 3 \quad .4 \quad .523$ POL

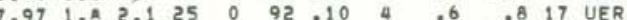

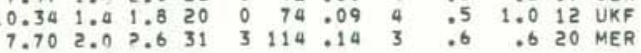

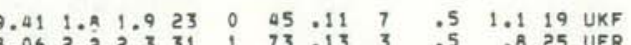

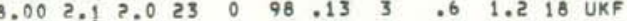

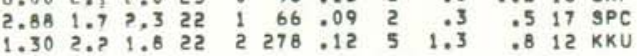
$\begin{array}{lllllllllllll}8.47 & 2.5 & 3.1 & 32 & 1 & 88 & .11 & 5 & .5 & .6 & 22 & \text { UER }\end{array}$

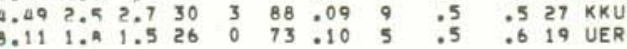




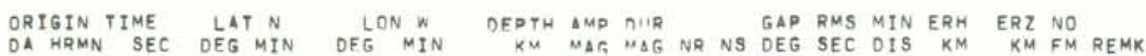

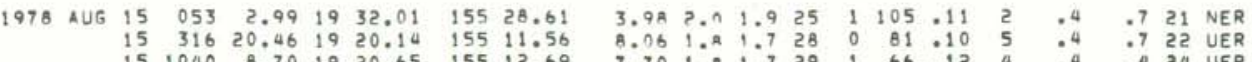
$151040 \quad 8.701920 .1515511 .56$ $15 \quad 2120 \quad 6.73 \quad 1923.96 \quad 15516.13$

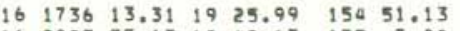
$16 \quad 2023 \quad 35.631809 .63 \quad 155 \quad 7.83$

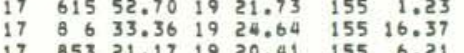

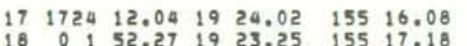
$18 \quad 0152.271923 .25 \quad 15517.18$ $18 \quad 2120 \quad 44.90 \quad 19 \quad 25.51 \quad 155 \quad 24.95$ $182343 \quad 10.0419 \quad 18,41 \quad 15513,35$

$\begin{array}{lllllll}19 & 212 & 29.11 & 19 & 55.90 & 155 & 21.54 \\ 19 & 523 & 25.26 & 19 & 32.71 & 155 & 37.43\end{array}$ $\begin{array}{lllllll}19 & 523 & 25.26 & 19 & 32.71 & 155 & 37.43 \\ 19 & 524 & 21.37 & 19 & 22.70 & 155 & 1.83\end{array}$ $\begin{array}{lrlllll}19 & 954 & 27.38 & 19 & 18.58 & 155 & 13.58 \\ 19 & 2018 & 14.78 & 19 & 21.32 & 155 & 25.70\end{array}$ $\begin{array}{llllllll}19 & 22 & 6 & 24.92 & 19 & 19.32 & 155 & 9.99\end{array}$ $\begin{array}{llrllll}20 & 948 & 6.25 & 19 & 26.95 & 154 & 55.99\end{array}$

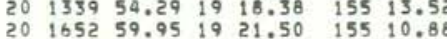

$21 \quad 115 \quad 59.74 \quad 19 \quad 19.35 \quad 155 \quad 8.03$ $92,44.86 \quad 19 \quad 19.84 \quad 155 \quad 8.75$ $\begin{array}{lrlllll}21 & 2114 & 24.87 & 19 & 25.47 & 155 & 24.51 \\ 22 & 012 & 58.68 & 19 & 23.06 & 155 & 17.14\end{array}$

$\begin{array}{lllllll}22 & 931 & 11.70 & 19 & 22.73 & 155 & 2.50\end{array}$ $22 \quad 1225 \quad 50.66 \quad 1921.19 \quad 155 \quad 7.83$

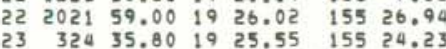
$\begin{array}{lllllll}23 & 1022 & 48.45 & 19 & 20.87 & 155 & 24.77 \\ 23 & 1148 & 53.12 & 19 & 19.82 & 155 & 8.16\end{array}$ $\begin{array}{lllllll}23 & 1757 & 24.55 & 19 & 19.57 & 155 & 11.31\end{array}$

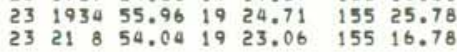
$\begin{array}{lllllll}24 & 150 & 39.90 & 19 & 19.91 & 155 & 11.71\end{array}$ $\begin{array}{llllllll}24 & 5 & 1 & 42.86 & 19 & 32.33 & 155 & 57.7 \\ 24 & 9 & 73 & 33.06 & 19 & 26.64 & 155 & 26.4\end{array}$

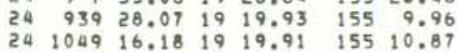

$\begin{array}{llllllll}24 & 17 & 99.54 & 19 & 23.14 & 155 & 16.99\end{array}$ $\begin{array}{lllllll}24 & 1947 & 8.56 & 19 & 23.77 & 155 & 15.74 \\ 24 & 2128 & 21.13 & 19 & 19.47 & 155 & 8.58\end{array}$

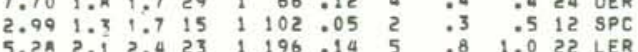

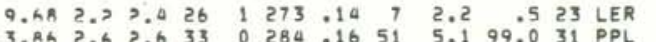

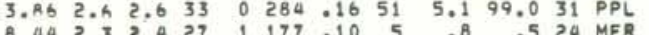

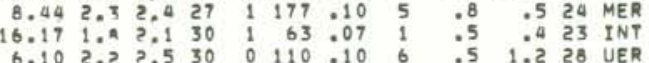
$\begin{array}{llllllllllll}3.25 & 1.9 & ? .1 & 14 & 0 & 105 & .08 & 2 & .4 & .5 & 14 & \mathrm{SPC}\end{array}$

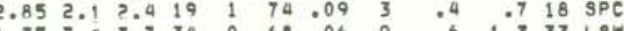

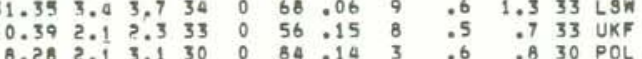
$\begin{array}{llllllllllll}0.52 & 2.9 & 3.1 & 34 & 2 & 189 & .13 & 20 & .9 & .7 & 29 & \mathrm{kKU}\end{array}$

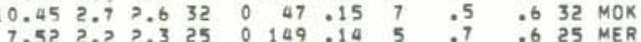

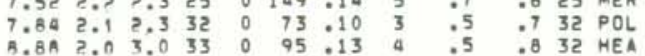
$\begin{array}{llllllllllllll}7.80 & 1.9 & 2.1 & 26 & 0 & 101 & .09 & 5 & .5 & .7 & 25 & \text { UER }\end{array}$

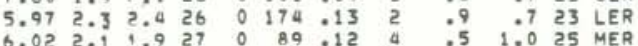
$\begin{array}{llllllllllll}0.28 & 2 . A & 2.9 & 36 & 1 & 76 & 11 & 3 & .6 & .5 & 30 & \text { POL } \\ 8.03 & 2.0 & 2.1 & 33 & 1 & 63 & .12 & 2 & .0 & .6 & 30 & \text { UER }\end{array}$

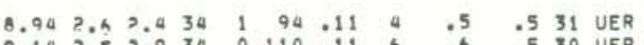

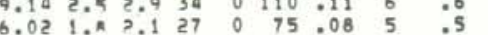
$\begin{array}{llllllllllll}0.97 & 3.0 & 3.0 & 37 & 1 & 33 & .14 & 8 & .4 & .5 & 33 & \text { UKF } \\ 2.73 & 1.4 & 1.8 & 17 & 2 & 66 & .07 & 3 & .3 & .5 & 13 & \text { SPC }\end{array}$

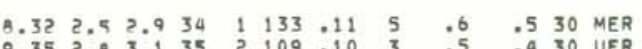

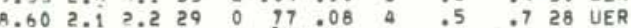

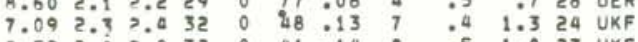
$\begin{array}{lllllllllll}8.52 & 2.0 & 5.0 & 32 & 0 & 41 & .14 & 8 & .5 & 1.0 & 23 \\ \text { UKF }\end{array}$

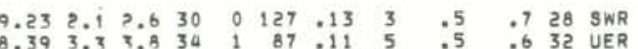
$9.22 \quad 2.5 \quad 3.131 \quad 0 \quad 95.12 \quad 5 \quad .6 \quad .530$ UER

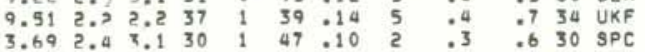
$\begin{array}{llllllllllll}8.2 B & 2.0 & 2.1 & 29 & 1 & 85 & .11 & 5 & .5 & .7 & 26 & \text { UER }\end{array}$

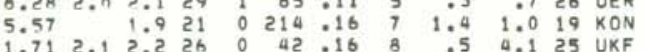
$\begin{array}{llllllllllll}1.71 & 2.1 & 2.2 & 26 & 0 & 42 & 16 & 8 & .5 & 4.1 & 25 & \text { UKF } \\ 7.44 & 1 . A & 2.1 & 28 & 0 & 86 & .09 & 4 & .5 & .9 & 28 & \text { UER }\end{array}$ $9,032,02.329,89.11$ 4 4 B 28 UER

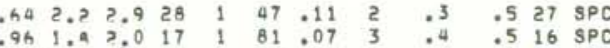

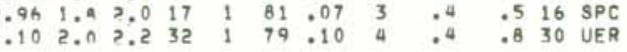

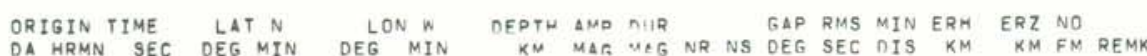

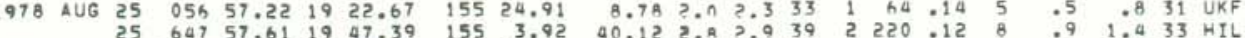

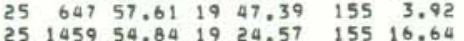

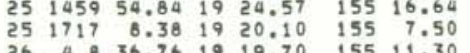
$26 \quad 720 \quad 19.45 \quad 19 \quad 17.65 \quad 155 \quad 21.70$ 26132434.011923 .75 155 16.90 2722748.91 19 $21.74 \quad 15512.7$ $27 \quad 316 \quad 51.33 \quad 1925.75 \quad 15524.49$

$\begin{array}{lllllll}27 & 633 & 4.70 & 19 & 23.04 & 155 & 16.91\end{array}$

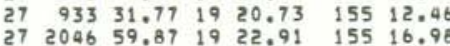
$28 \quad 9821.65 \quad 1918.61 \quad 155 \quad 13.95$ $28 \quad 1525 \quad 18.491919 .85 \quad 155 \quad 10.55$ $28 \quad 2025 \quad 42.04 \quad 1920.25 \quad 155 \quad 12.72$ $29033 \quad 36.9019 \quad 18.31 \quad 155 \quad 15.82$

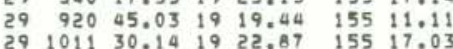

$29 \begin{array}{lllll}1144 & 8.82 \quad 19 \quad 19.05 \quad 155 \quad 11.78\end{array}$

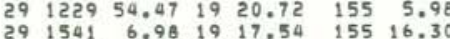

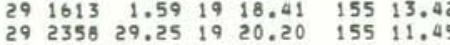

$\begin{array}{llllllll}30 & 11 & 92.58 & 19 & 19.36 & 155 & 7.46\end{array}$ $30124714.8919 .19 .33 \quad 15512.08$

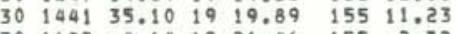
$\begin{array}{lllllll}30 & 1627 & 4.14 & 19 & 21.46 & 155 & 2.72\end{array}$

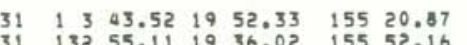
$3132446.6419-19.53 \quad 15513.16$ $\begin{array}{lllllll}31 & 13 & 20.97 & 18 & 58.73 & 155 & 29.29\end{array}$

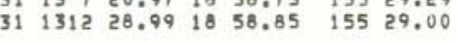

$\begin{array}{lllllll}31 & 1322 & 54.96 & 19 & 19.42 & 155 & 12.56\end{array}$ $\begin{array}{lllllll}31 & 1414 & 53.51 & 18 & 59.56 & 155 & 28.67\end{array}$

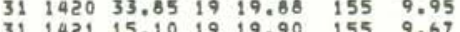
$\begin{array}{rrrrrrr}31 & 2146 & 29.09 & 19 & 30.09 & 155 & 12.10 \\ 31 & 2232 & 6.84 & 19 & 23.11 & 155 & 32.75\end{array}$

SEP $1 \quad 4 \quad 1 \quad 25.15 \quad 1920.38 \quad 155 \quad 6.37$

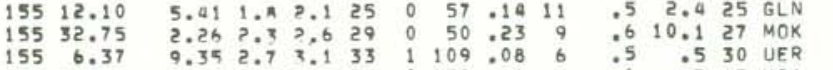

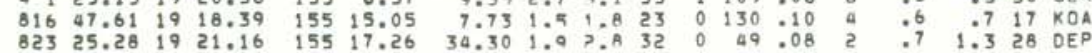

$\begin{array}{llllllllllllllllllll}1 & 1458 & 1.76 & 18 & 53.52 & 155 & 31.29 & 37.11 & 2.1 & 2.3 & 23 & 2 & 269 & .07 & 18 & 1.5 & 2.0 & 19 & \text { DIS } \\ 1 & 1748 & 17.79 & 19 & 30.30 & 155 & 6.01 & 7.74 & 2 & 2.1 & 27 & 0 & 108 & .09 & 6 & .5 & 1.0 & 24 & \text { UER }\end{array}$

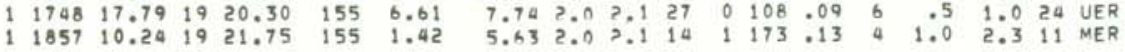


YEAR MON ORIGIN TIME OARM LAT N 1978 SEP

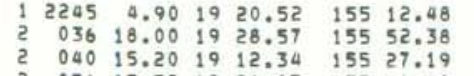

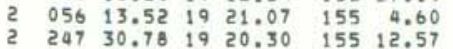

$2 \quad 619 \quad 35.80 \quad 1921.74 \quad 155 \quad 18.11$

$175 \quad 4.001932 .46 \quad 155 \quad 16.84$

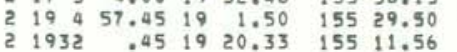

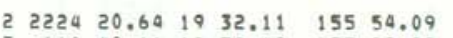

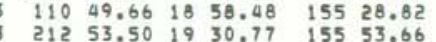

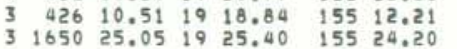

$\begin{array}{lllllll}3 & 1711 & 6.54 & 19 & 22.98 & 155 & 16.91\end{array}$

4 \begin{tabular}{l}
$345 \quad 53.36 \quad 19 \quad 33.99 \quad 155 \quad 41.78$ \\
\hline
\end{tabular}

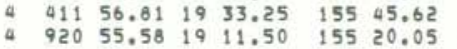

a $2014 \quad 44.10 \quad 19 \quad 19.31 \quad 155 \quad 8.49$

42011
5 $247.231918 .83 \quad 15515.12$

$\begin{array}{lllllll}5 & 047 & 17.38 & 19 & 20.55 & 155 & 6.44 \\ 5 & 428 & 18.61 & 19 & 19.06 & 155 & 11.86\end{array}$

$\begin{array}{llll}1026 & 46.87 \quad 19 \quad 20.13 \quad 155 \quad 13.60\end{array}$

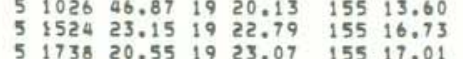

$\begin{array}{lllllll}5 & 1738 & 20.55 & 19 & 23.07 & 155 & 17.01 \\ 5 & 1939 & 43.64 & 19 & 20.74 & 155 & 4.13\end{array}$

$629 \quad 7.14 \quad 19 \quad 19.58 \quad 155 \quad 11.16$

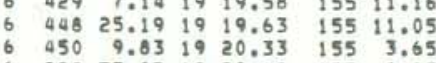

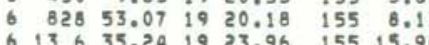

$\begin{array}{lllllll}6 & 1414 & 30.06 & 19 & 24.04 & 155 & 24.21 \\ 6 & 2349 & 40.72 & 19 & 19.61 & 155 & 11.74\end{array}$

$\begin{array}{rrrrrrr}6 & 2349 & 40.72 & 19 & 19.61 & 155 & 11.74 \\ 7 & 214 & 28.38 & 19 & 21.36 & 155 & 15.36\end{array}$

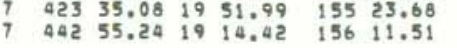

$\begin{array}{rrrrrrr}7 & 758 & 33.90 & 19 & 19.87 & 155 & 12.16 \\ 7 & 1137 & 46.03 & 19 & 21.76 & 155 & 4.05\end{array}$

165653.3919
7

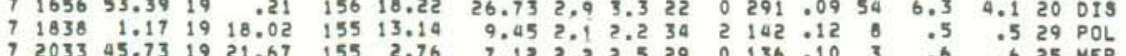

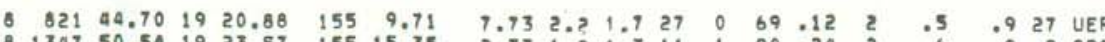

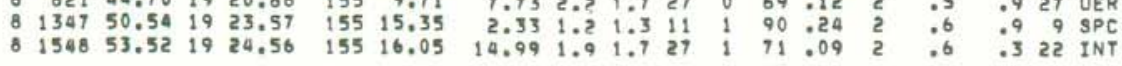

$\begin{array}{lllllllllllll}8.03 & 1.5 & .8 & 25 & 0 & 69 & .12 & 4 & .5 & .8 & 21 & \text { UER } \\ 8.41 & 1.5 & 17 & 3 & 126 & .21 & 5 & 1.0 & 1.0 & 11 & \text { KON }\end{array}$

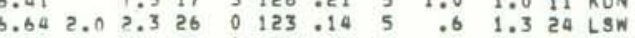
$\begin{array}{lllllllllllll}4.99 & 1.8 & 2.1 & 27 & 0 & 95 & .11 & 4 & .6 & 1.0 & 18 & \text { MER } \\ 7.98 & 1.5 & 1.8 & 23 & 0 & 71 & .11 & 4 & .5 & .7 & 19 & \text { UER }\end{array}$ $\begin{array}{llllllllllll}3.67 & 1.9 & 0.1 & 23 & 1 & 49 & .09 & 3 & .3 & .8 & 19 & \mathrm{KOA}\end{array}$

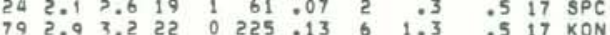
$\begin{array}{llllllllllll}8.82 & 1.9 & 21 & 4 & 182 & .19 & 6 & .7 & .7 & 15 & \text { KON } \\ 38.77 & 2.7 & 2.7 & 31 & 0 & 225 & .05 & 19 & 1.5 & 2.4 & 29 & \text { OI }\end{array}$

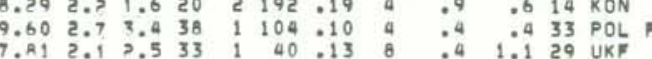
$\begin{array}{lllllllllllllll}2.26 & 1.2 & 1.3 & 13 & 1 & 69 & .08 & 2 & .4 & .6 & 10 & \mathrm{kOA}\end{array}$ $\begin{array}{lllllllllllll}91 & 2.1 & 2.5 & 27 & 2 & 92 & .11 & 10 & .5 & .9 & 21 & \text { MOK }\end{array}$ $\begin{array}{llllllllllll}3.75 & .7 & 2.0 & 20 & 2 & 86 & .12 & 5 & .6 & 1.7 & 18 & \text { KON } \\ 5.6 & 35 & 1 & 175 & .07 & 12 & .9 & 1.6 & 32 & \text { HLP }\end{array}$

$\begin{array}{llllllllllll}.57 & 3.1 & 7.3 & 36 & 1 & 82 & 09 & 4 & .5 & .4 & 34 & \text { UER }\end{array}$

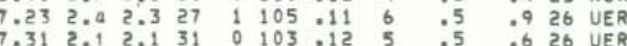

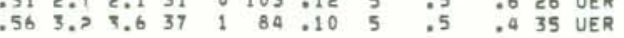
$\begin{array}{llllllllllll}.09 & 3.7 & 0.2 & 34 & 0 & 60 & .09 & 5 & .4 & .4 & 30 & \text { UER } \\ 3.89 & 1.5 & 1.8 & 10 & 1 & 78 & .12 & 2 & .5 & .8 & 7 & \text { KOA }\end{array}$

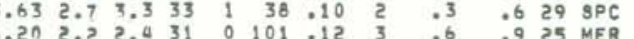

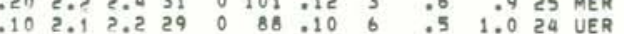

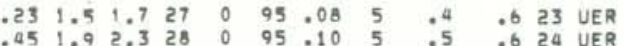

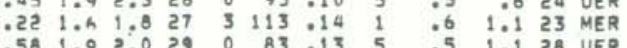

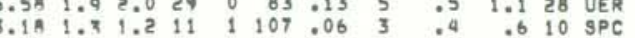
$\begin{array}{llllllllllll}.58 & 2.1 & 2.3 & 32 & 0 & 41 & -13 & 7 & .5 & .6 & 26 & \text { UKF } \\ .5 & 2.3 & 28 & 0 & 91 & -12 & 6 & .5 & .6 & 27 & \text { UER }\end{array}$ $\begin{array}{rrrrrrrrrrrr}.41 & 1: 9 & 1.8 & 23 & 2 & 216 & 12 & 15 & 1.2 & 1.4 & 18 & \text { LSW } \\ 6.76 & 1.0 & 1.3 & 24 & 0 & 78 & .12 & 4 & .5 & .8 & 20 & \text { UER }\end{array}$ $.112 .92 .628 \quad 0 \quad 199.09$ 6 $1.2 \quad .4026$ KKU

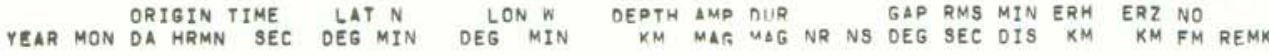

1978 SEP \& $18 \quad 5 \quad 45.28 \quad 1923.70 \quad 155 \quad 16.01$

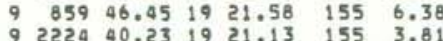
100396.25 19 21.72 155 29.94 $10 \quad 334 \quad 56.58 \quad 1923.46 \quad 15458.11$

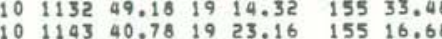
$\begin{array}{lllllll}10 & 1340 & 34.50 & 19 & 21.58 & 155 & 26.40 \\ 10 & 1833 & 55.86 & 19 & 19.10 & 155 & 11.01\end{array}$ $102114 \quad 54.31 \quad 1920.84 \quad 155 \quad 3.10$ $\begin{array}{llllllll}11 & 0 & 47.87 & 19 & 23.06 & 155 & 16.93 \\ 11 & 121 & 57.18 & 18 & 58.08 & 155 & 20.62\end{array}$

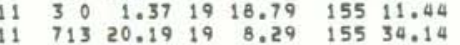
$\begin{array}{lllllll}11 & 731 & 55.37 & 19 & 23.60 & 155 & 15.19\end{array}$ i1 $1150 \quad 36.441920 .01 \quad 15512.65$

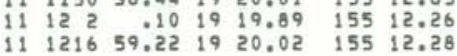
$\begin{array}{lllllll}11 & 1219 & 51.00 & 19 & 20.71 & 155 & 12.02\end{array}$ $112016 \quad 6.111920 .00 \quad 155 \quad 6.52$

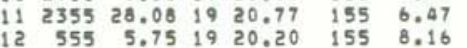

$\begin{array}{lllllll}12 & 712 & 13.67 & 19 & 24.53 & 155 & 17.18\end{array}$ $12 \quad 71428.481923 .40 \quad 15459.00$ $\begin{array}{lllllll}12 & 729 & 1.36 & 19 & 29.90 & 155 & 42.36 \\ 13 & 330 & 10.94 & 19 & 19.89 & 155 & 8.24\end{array}$ $1353149.531924 .56 \quad 15524.79$ $\begin{array}{rrrrrrrr}13 & 12 & 1 & 55.94 & 19 & 20.53 & 155 & 3.92 \\ 13 & 1210 & 2.11 & 19 & 23.50 & 155 & 2.27\end{array}$ $\begin{array}{lllllll}13 & 1558 & 32.54 & 19 & 21.61 & 155 & 2.53 \\ 13 & 1627 & 9.44 & 19 & 24.70 & 155 & 25.09\end{array}$

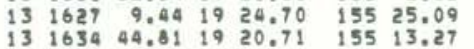
$\begin{array}{lllllll}13 & 1636 & 16.86 & 19 & 20.33 & 155 & 12.94\end{array}$ $\begin{array}{lllllll}13 & 1818 & 4.35 & 19 & 25.23 & 155 & 17.04 \\ 13 & 2142 & 21.17 & 19 & 23.12 & 155 & 16.03\end{array}$

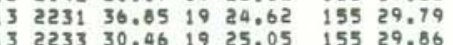

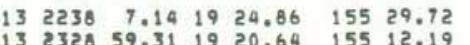

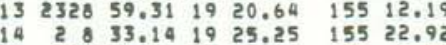
1425321.41 19 22.82 1552.15 $\begin{array}{rrrrrrr}14 & 833 & 47.98 & 19 & 22.02 & 155 & 1.96\end{array}$

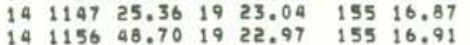

$\begin{array}{lllllllllll}3.39 & 1.92 .1 & 20 & 1 & 73 & .10 & 2 & .4 & .6 & 18 & \text { SPC }\end{array}$

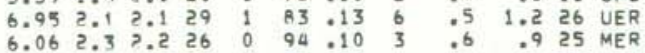

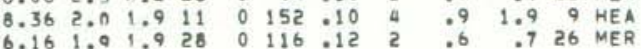
$\begin{array}{lllllllllll}5.45 & 2.0 & 1.9 & 28 & 1 & 194 & .17 & 3 & 1.1 & .925 & 25 \\ \text { LER }\end{array}$

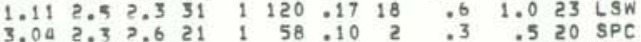

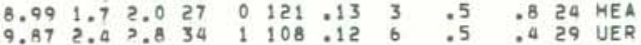
$\begin{array}{llllllllllll}6.38 & 1.9 & 2.3 & 26 & 0 & 1222 & .13 & 2 & .7 & .8 & 24 & \text { MER }\end{array}$

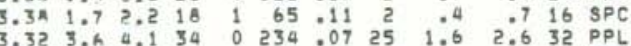
$\begin{array}{rrrrrrrrrrrr}5.93 & 1.8 & 2.1 & 24 & 1 & 115 & 11 & 5 & .6 & 1.5 & 18 & \text { POL } \\ 2.67 & 2.8 & & 26 & 1 & 217 & .20 & 11 & 1.9 & 2.3 & 20 & \text { HEA }\end{array}$ $\begin{array}{lllllllllllll}3.31 & 2.6 & 2.7 & 18 & 1 & 78 & .12 & 3 & .4 & .6 & 10 & \text { SPC F } \\ 4.23 & 12.6 & .8 & 16 & 0 & 87 & 09 & 3 & .5 & 1.4 & 15 & \text { SWR }\end{array}$ $\begin{array}{llllllllllll}7.81 & 2.1 & 2.5 & 32 & 2 & 75 & .13 & 5 & .4 & 7 & 26 & \text { UER }\end{array}$

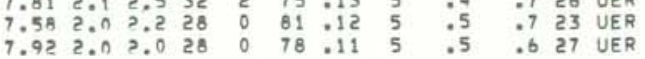
$\begin{array}{llllllllllll}7.38 & 1.9 & 2.0 & 28 & 0 & 70 & .12 & 4 & .5 & .8 & 23 & \text { UER }\end{array}$

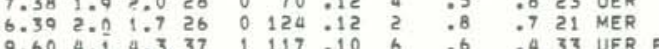

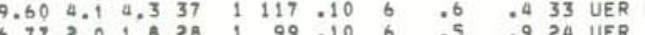

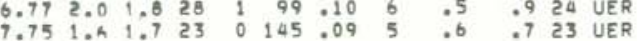

$\begin{array}{lllllllllllll}1.25 & 1.1 & 1.7 & 12 & 1 & 57 & .09 & 1 & .4 & .3 & 10 & \mathrm{SPC}\end{array}$

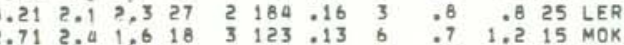

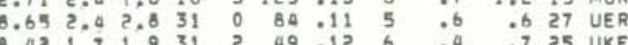

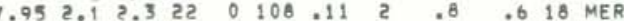

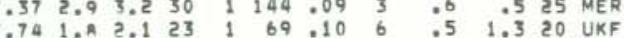

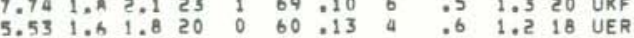

$\begin{array}{llllllllllll}0.20 & 2.1 & 2.5 & 27 & 0 & 67 & .11 & 4 & .5 & .7 & 26 & \text { UER }\end{array}$

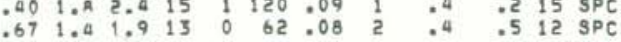

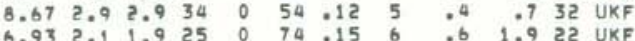
$\begin{array}{llllllllllll}10.00 & 2.9 & 3.0 & 34 & 2 & 54 & 010 & 6 & .4 & .6 & 28 & \text { UKF } \\ 7.45 & 1.9 & .9 & 33 & 0 & 70 & .12 & 4 & .5 & .6 & 28 & \text { UER }\end{array}$ $9.872 .32 .432 \quad 1 \quad 38.12 \quad 6 \quad .40 .631$ UKF $5.192 .3 ? .123$ 0 172.13 a $.6 \quad 1.023$ MER $\begin{array}{rrrrrrrrrrrr}6.24 & 2.0 & 1.7 & 24 & 0 & 155 & .15 & 4 & .7 & 1.1 & 22 & \text { MER } \\ 2.87 & 1.3 & 1.4 & 12 & 1 & 65 & .05 & 2 & .4 & .5 & 11 & 9 P C \\ 3.01 & 1.7 & 2.0 & 15 & 0 & 70 & .06 & 2 & .3 & .5 & 15 & \text { KOA }\end{array}$

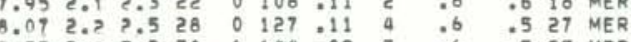


HE

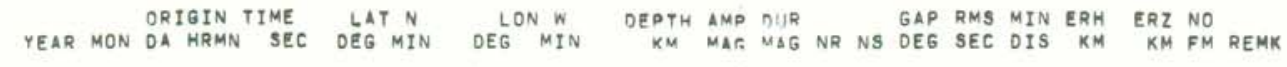
1978 SEP 15 O $00622.57 \quad 1920.73 \quad 155 \quad 6.44$ $15 \quad 332 \quad 1.731919 .89$ 155 11.32 $\begin{array}{lllllll}15 & 514 & .74 & 19 & 25.42 & 155 & 16.56 \\ 15 & 515 & 53.10 & 19 & 25.38 & 155 & 16.61\end{array}$ $\begin{array}{lllllll}15 & 715 & 58.31 & 20 & 15.24 & 156 & .24 \\ 15 & 723 & 55.65 & 19 & 25.33 & 155 & 16.30\end{array}$

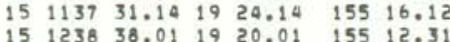
$\begin{array}{lllllll}15 & 1238 & 38.01 & 19 & 20.01 & 155 & 12.31 \\ 15 & 2330 & 37.73 & 19 & 16.94 & 155 & 22.58\end{array}$ $\begin{array}{llllllll}16 & 2 & 1 & 29.59 & 19 & 20.87 & 155 & 6.08\end{array}$ $\begin{array}{lllllll}16 & 630 & .61 & 19 & 23.97 & 155 & 15.68\end{array}$ $\begin{array}{rrrrrrr}16 & 1529 & 58.89 & 19 & 24.91 & 155 & 25.74 \\ 16 & 1747 & 1.85 & 19 & 20.12 & 155 & 11.68\end{array}$ 1621 2 12.47 19 $19.50 \quad 1559.72$

$\begin{array}{rrrrrrr}16 & 2141 & 5.36 & 19 & 22.17 & 155 & 3.42 \\ 17 & 055 & 35.29 & 19 & 20.22 & 155 & 10.34\end{array}$ $17 \quad 3743.551923 .58 \quad 15516.80$ $\begin{array}{llllllll}17 & 351 & 46.60 & 19 & 21.77 & 155 & .24 \\ 17 & 9 & 6 & 21.91 & 19 & 23.45 & 155 & 16.97\end{array}$ $\begin{array}{rrrrrrr}17 & 921 & 40.58 & 19 & 21.42 & 155 & 7.06 \\ 17 & 1656 & 52.31 & 19 & 19.69 & 155 & 10.97\end{array}$

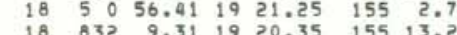

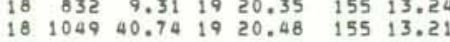
$\begin{array}{lllllll}18 & 1121 & 6.23 & 19 & 23.08 & 155 & 16.90 \\ 18 & 1730 & 1.13 & 19 & 20.87 & 155 & 2.84\end{array}$

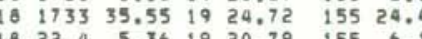
$\begin{array}{rrrrrrrr}18 & 22 & 4 & 5.36 & 19 & 20.79 & 155 & 6.16 \\ 18 & 23 & 3 & 13.56 & 19 & 17.04 & 155 & 23.82\end{array}$ $\begin{array}{lllllll}18 & 2344 & 29.20 & 19 & 23.38 & 155 & 16.77 \\ 18 & 2346 & 53.33 & 19 & 23.45 & 155 & 16.93\end{array}$ $18234653.331923 .45 \quad 15516.93$ 1902416.73 19 $21.04 \quad 155 \quad 16.93$ $\begin{array}{llllllll}19 & 3 & 4 & 59.25 & 19 & 19.58 & 155 & 26.71\end{array}$

$\begin{array}{lllllll}19 & 319 & 34.02 & 19 & 23.06 & 155 & 16.97 \\ 19 & 843 & 44.06 & 19 & 2.59 & 155 & 41.46\end{array}$ $\begin{array}{rrrrrrr}19 & 843 & 44.06 & 19 & 2.59 & 155 & 41.96 \\ 19 & 1048 & 10.75 & 19 & 18.54 & 155 & 10.92\end{array}$ $\begin{array}{llllllll}19 & 1048 & 10.75 & 19 & 18.54 & 155 & 10.92 \\ 19 & 1242 & 23.97 & 19 & 21.79 & 155 & 12.72\end{array}$ $\begin{array}{llllllll}19 & 18 & 1 & 25.11 & 19 & 25.82 & 155 & 24.90\end{array}$

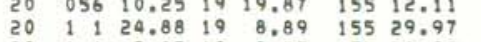
$\begin{array}{lllllllll}20 & 1 & 6 & 2.97 & 19 & 8.75 & 155 & 31.03 \\ 20 & 410 & 24.47 & 19 & 21.66 & 155 & 28.10\end{array}$ $\begin{array}{lllllll}20 & 1028 & 56.03 & 19 & 18.91 & 155 & 11.56\end{array}$ $201217 \quad 56.73 \quad 1923.12 \quad 155 \quad 3.26$ $\begin{array}{cccccccccccc}8.73 & 2.4 & 2.7 & 31 & 0 & 101 & .10 & 6 & .5 & .6 & 30 & \text { UER } \\ 8.738 & .7 & 3.7 & 29 & 0 & 98 & .09 & 6 & .5 & .6 & 20 & \text { UER }\end{array}$

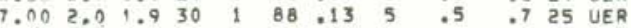

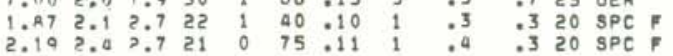

$16.493 .0 \times .031 \quad 1307 \quad .2027 \quad 4.940 .926 \quad 019$.

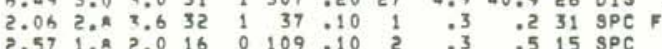

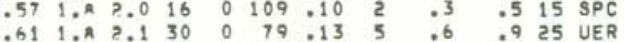

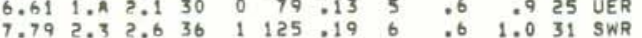
$\begin{array}{rrrrrrrrrrrr}6.87 & 1.9 & 1.9 & 29 & 2 & 100 & .12 & 6 & .5 & 1.0 & 23 & \text { UER } \\ 3.67 & 2.9 & 3.2 & 35 & 1 & 60 & 011 & 3 & .5 & .5 & 33 & \text { SPC }\end{array}$

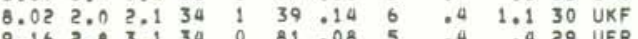

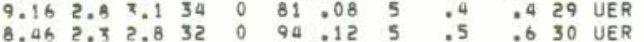

$\begin{array}{llllllllllll}6.72 & 2.6 & 5.1 & 30 & 0 & 112 & .10 & 4 & .4 & .6 & 29 & \text { MER } \\ 6.40 & 1.5 & 09 & 28 & 0 & 82 & 11 & 4 & .5 & .8 & 21 & \text { UER }\end{array}$

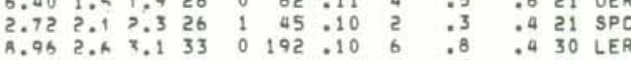

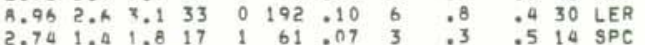
$\begin{array}{lllllllllllll}4.29 & 1 . A & 0.0 & 30 & 0 & 81 & .14 & 5 & .6 & 1.7 & 25 & \text { UER } \\ 5.26 & 1 . A & 1.8 & 30 & 1 & 93 & 14 & 5 & .5 & 1.2 & 23 & \text { UER }\end{array}$

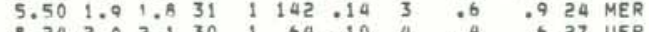

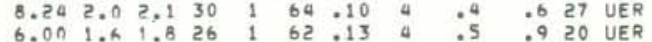
$\begin{array}{rrrrrrrrrrrr}2.83 & 1.3 & .9 & 9 & 1 & 86 & .10 & 2 & .5 & .7 & 8 & \text { SPC } \\ 5.78 & 2.0 & 1.8 & 27 & 1 & 139 & : 12 & 2 & .6 & .8 & 25 & \text { MER }\end{array}$ $10.252 .3 \quad 2.034 \quad 1 \quad 33.13$

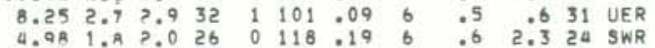

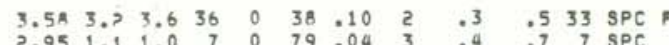

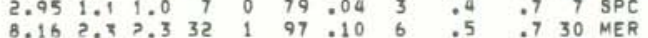

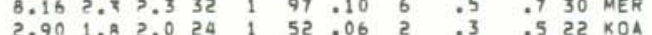

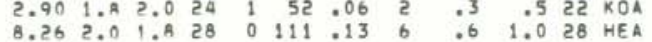
$\begin{array}{rrrrrrrrrrrr}2.15 & 1.1 & .7 & 8 & 0 & 96 & .07 & 2 & .4 & .8 & 7 & \text { SPC } \\ 43.14 & 3.1 & 0.4 & 36 & 2 & 165 & .08 & 7 & 1.1 & 2.0 & 34 & \text { HEA }\end{array}$

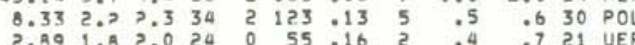

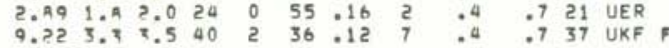
$\begin{array}{llllllllllllll}5.90 & 1.5 & 1.5 & 31 & 2 & 57 & .19 & 8 & .4 & 2.0 & 29 & \text { UKF } \\ 8.30 & 2.1 & 0.5 & 31 & 1 & 83 & .10 & 5 & .4 & .6 & 28 & \text { UER }\end{array}$ $39.72 \quad 2.22 .836$ a 152.08 4 0.8 . 934 LSW L

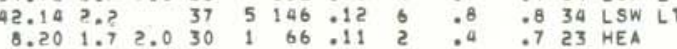

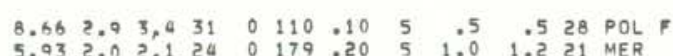

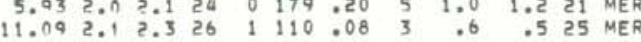

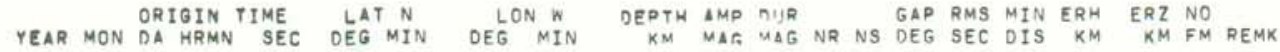

1978 SEP $20132026.99 \quad 1930.67 \quad 155 \quad 52.63$ $2105 \quad 46.43 \quad 1921.70 \quad 155 \quad 14.84$

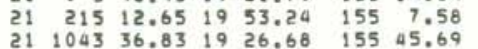
$21 \quad 1428 \quad 14.37 \quad 19 \quad 17.45 \quad 155 \quad 16.96$

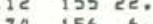
$212027 \quad 55.591919 .37$ 155 11.32 22 $\quad 153 \quad 51.08 \quad 19 \quad 19.92 \quad 155 \quad 11.18$ $\begin{array}{lllllll}22 & 153 & 51.08 & 19 & 19.92 & 155 & 11.16 \\ 22 & 12 & 22.37 & 19 & 19.50 & 155 & 10.05\end{array}$ $221456 \quad 35.001919 .81 \quad 155 \quad 11.11$

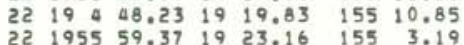

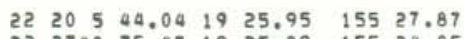

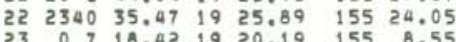
$\begin{array}{llllllll}23 & 0 & 7 & 10.42 & 19 & 20.19 & 155 & 8.55\end{array}$ $\begin{array}{lllllll}23 & 7 & 5 & 57.31 & 30.93 & 19 & 23.75 \\ 21.55 & 155 & 24.74\end{array}$

$\begin{array}{lllllll}23 & 714 & 17.99 & 19 & 25.90 & 155 & 24.02\end{array}$

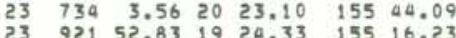

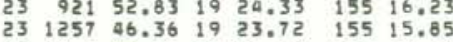
2314435.18 19 21.40 $155 \quad 7.1$

$\begin{array}{lllllll}23 & 1636 & 22.35 & 19 & 20.46 & 155 & 13.05\end{array}$ $\begin{array}{lllllll}23 & 2159 & 5.24 & 19 & 19.14 & 155 & 11.61 \\ 24 & 114 & 41.62 & 19 & 20.38 & 155 & 13.29\end{array}$ $24 \quad 212 \quad 11.391927 .15 \quad 15528.09$

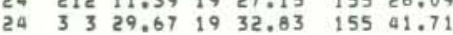

$\begin{array}{lllllll}24 & 555 & 48.94 & 19 & 22.90 & 155 & 17.02 \\ 24 & 911 & 57.16 & 19 & 20.19 & 155 & 11.29\end{array}$ $\begin{array}{lllllll}24 & 911 & 57.16 & 19 & 20.19 & 155 & 11.29 \\ 24 & 940 & 42.38 & 19 & 23.42 & 155 & 23.61\end{array}$

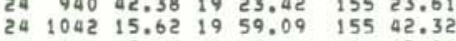
$24 \quad 1055 \quad 55.73 \quad 19 \quad 23.96 \quad 155 \quad 15.98$

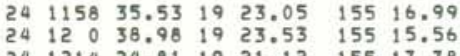
24122409.91 19 22.96 15516.9 $24 \quad 1254 \quad 38.87 \quad 1922.81 \quad 155 \quad 16.96$ $24 \begin{array}{lllll}1530 & 53.02 \quad 19 & 23.36 & 155 & 25.0\end{array}$

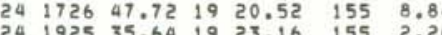
24222658.93 19 20.70 155 13.26 $242320.461922 .92 \quad 155 \quad 16.80$

$\begin{array}{llllllll}25 & 1 & 3 & 18.28 & 19 & 47.47 & 155 & 6.78\end{array}$ $\begin{array}{lllllll}25 & 114 & 54.73 & 19 & 21.26 & 155 & 1.02 \\ 25 & 128 & 21.50 & 19 & 20.12 & 155 & 13.19\end{array}$ $\begin{array}{lllllllllllllll}9.63 & 3 & \times & 3 & 30 & 0 & 129 & .17 & 5 & .7 & .5 & 26 & K O N\end{array}$

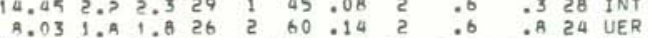

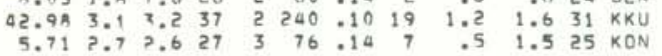
$\begin{array}{llllllllllll}8.91 & 2.7 & 2.6 & 35 & 2 & 133 & .14 & 3 & .6 & .6 & 30 & \mathrm{KOA}\end{array}$

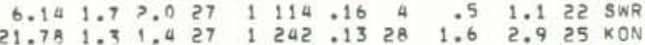
$9.90 \quad 2.73 .135$ 0 99.0960 . 5 .4 33 UER

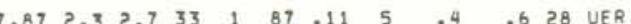

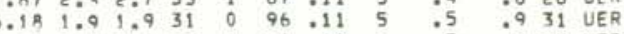

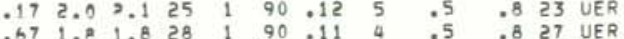

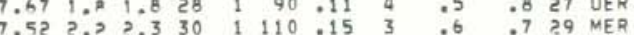

$\begin{array}{llllllllllll}1.25 & 2.9 & \times .0 & 36 & 0 & 34 & .13 & 6 & .4 & .6 & 33 & \text { UKF }\end{array}$

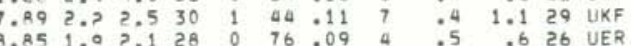

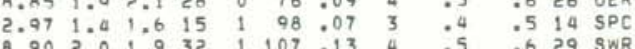
$\begin{array}{llllllllllll}7.46 & 2.1 & 2.4 & 28 & 0 & 44 & .13 & 7 & .5 & 1.4 & 26 & \text { UKF }\end{array}$

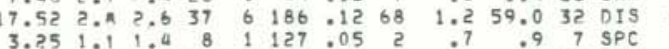

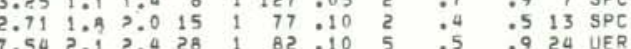

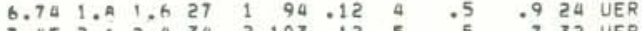

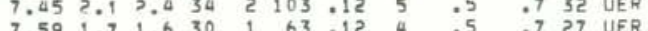
$\begin{array}{llllllllllll}10.04 & 2.1 & 2.2 & 37 & 2 & 38 & .12 & 9 & .4 & .7 & 32 & \text { UKF } \\ 3.94 & 1.5 & 1.7 & 23 & 2 & 87 & .14 & 8 & .4 & 3.0 & 23 & 40 K\end{array}$

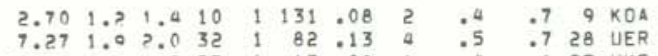

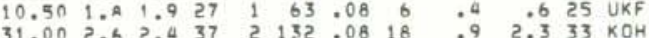
$2,701,7 ? .020 \quad 1 \quad 81.11 \quad 3 \quad .40 .518$ SPC

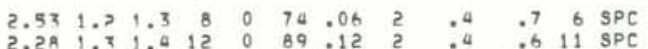

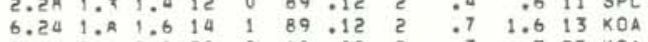

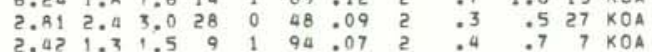

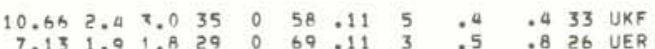

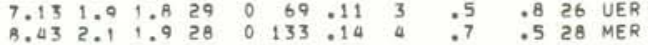

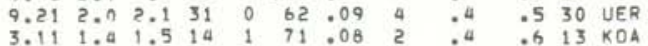

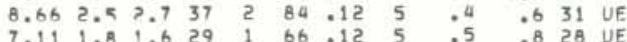
$\begin{array}{lllllllllllll}41.28 & 1.5 & 1.6 & 37 & 5 & 199 & .11 & \text { B } & .9 & 1.3 & 36 & \mathrm{HIL}\end{array}$ 
ORIGIN TIME
OA HRMN SEC DET N

1978 SEP $25 \quad 149 \quad 54.75 \quad 1922.79 \quad 155 \quad 17.05$ 22.89
155
14.95 2583 $25 \quad 1131 \quad 42.04 \quad 1923.16 \quad 155 \quad 15.18$ $25133013.541919 .51 \quad 155 \quad 7.89$

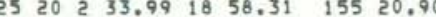

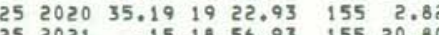
$252028 \quad 21.14 \quad 1921.60 \quad 155 \quad 7.86$ $25 \quad 233531.51 \quad 1920.11 \quad 155 \quad 10.97$ $\begin{array}{llllllll}26 & 016 & 16.29 & 19 & 24.05 & 155 & 17.39\end{array}$ $\begin{array}{lllllll}26 & 514 & 39.39 & 19 & 24.79 & 155 & 29.38 \\ 26 & 650 & 29.76 & 19 & 20.17 & 155 & 11.32\end{array}$ $\begin{array}{lllllll}26 & 951 & 3.16 & 19 & 21.24 & 155 & 2.06\end{array}$

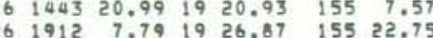
$\begin{array}{rrrrrrr}26 & 1935 & 44.22 & 19 & 26.48 & 155 & 23.08 \\ 27 & 59 & 16.10 & 19 & 19.98 & 155 & 8.97\end{array}$ $\begin{array}{lllllll}27 & 1443 & 38.17 & 19 & 23.06 & 155 & 16.67\end{array}$ $\begin{array}{lllllll}27 & 1458 & 57.45 & 19 & 14.95 & 155 & 1.59 \\ 27 & 16 & 46.38 & 19 & 19.07 & 155 & 16.16\end{array}$

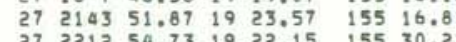

$\begin{array}{llllllll}28 & 2 & 6 & 21.12 & 19 & 23.34 & 155 & 16.99\end{array}$

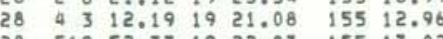

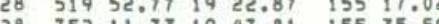

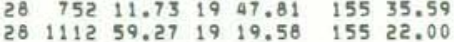
$\begin{array}{lllllll}28 & 1137 & 30.06 & 19 & 22.80 & 155 & 16.75 \\ 28 & 1538 & 20.19 & 19 & 22.99 & 155 & 17.03\end{array}$ $\begin{array}{lllllll}28 & 1544 & 33.90 & 19 & 23.13 & 155 & 17.09\end{array}$ $282215 \quad 11.75$ 19 21.46 $155 \quad 25.86$

$\begin{array}{lllllll}29 & 125 & 51.20 & 19 & 59.59 & 155 & 27.96\end{array}$ $\begin{array}{lrlllll}29 & 927 & 41.27 & 19 & 23.20 & 155 & 16.76 \\ 29 & 1316 & 54.29 & 19 & 23.08 & 155 & 13.79\end{array}$ $291627 \quad 58.341922 .31 \quad 155 \quad 5.72$

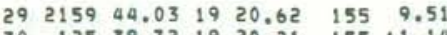

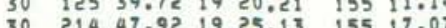
$30 \quad 343 \quad 36.83 \quad 19 \quad 19.01 \quad 155 \quad 23.76$

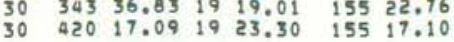
$\begin{array}{lllllll}30 & 1118 & 21.91 & 19 & 27.31 & 155 & 51.76\end{array}$

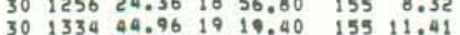

$\begin{array}{lllllllllllll}3.35 & 1.7 & 1.2 & 13 & 1 & 78 & .12 & 2 & .4 & .8 & 12 & K O A\end{array}$ $3.0415,513$ o $57.10 ? 230.612$ UER

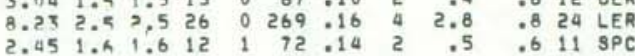
$\begin{array}{lllllllllllll}8.96 & 3.1 & 7.3 & 36 & 1 & 98 & .09 & 4 & .5 & .4 & 33 & \text { UER } \\ 5.27 & 2.1 & 09 & 24 & 1 & 190 & 015 & 3 & 09 & 10 & 20 & \text { LER }\end{array}$

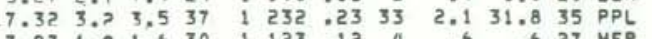

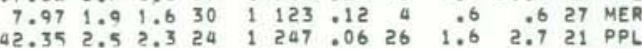

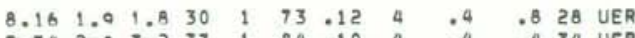

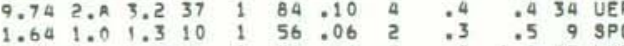
$\begin{array}{rrllllllllll}8.14 & 2.1 & .9 & 31 & 0 & 51 & .10 & 5 & .4 & 1.1 & 27 & \text { UKF } \\ 6.90 & 2.0 & 1.9 & 30 & 1 & 82 & .11 & 4 & .5 & .7 & 29 & \text { UER }\end{array}$ $\begin{array}{llllllllllll}5.48 & 2 . n & 2.0 & 25 & 1 & 166 & .14 & 3 & .6 & .8 & 23 & \text { MER }\end{array}$

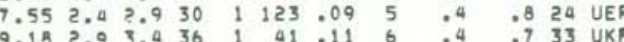

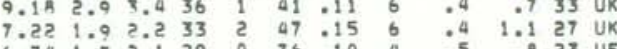

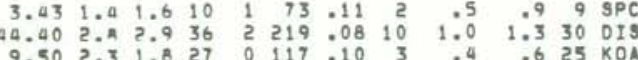

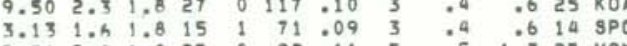
$\begin{array}{llllllllllll}2.18 & 1.1 & 1.5 & 6 & 0 & 92 & .04 & 3 & .4 & .8 & 6 & 9 P C\end{array}$

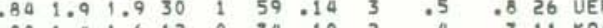

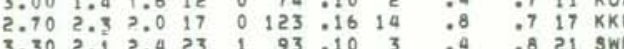

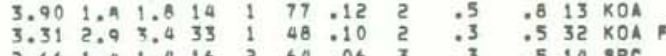

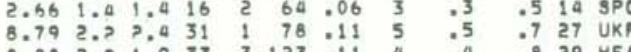
$\begin{array}{llllll}36 & 1.0 & 34.5 & 32 & \mathrm{KKU} & 0\end{array}$

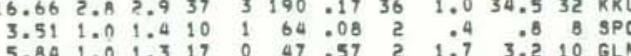

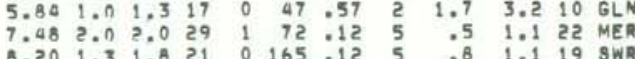

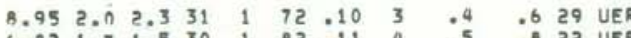

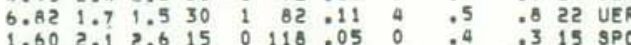

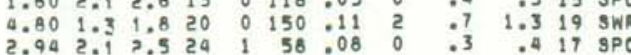

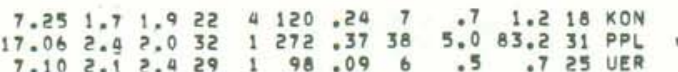

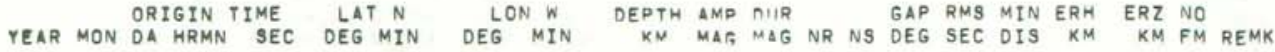

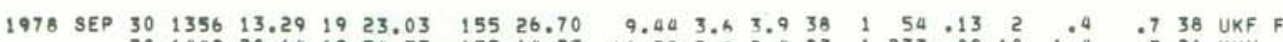

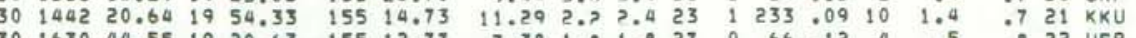

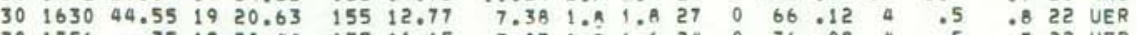

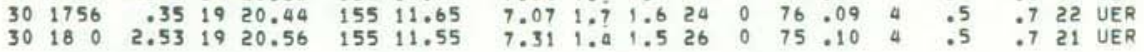

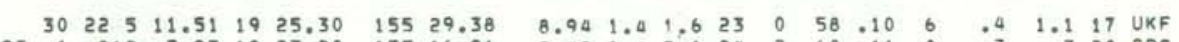

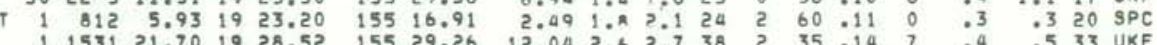

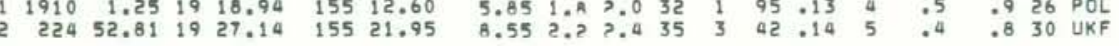

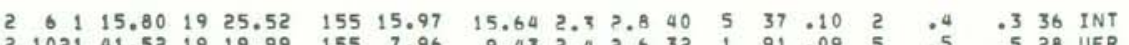

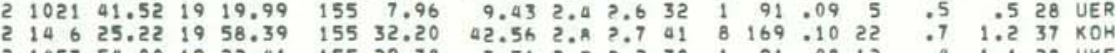

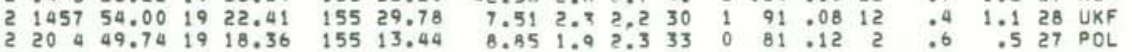
$\begin{array}{lllllll}3 & 431 & 56.20 & 19 & 20.07 & 155 & 12.79 \\ 3 & 638 & 21.88 & 19 & 23.82 & 155 & 16.11\end{array}$ $3 \begin{aligned} & 715 \\ & 3\end{aligned}$ $\begin{array}{lllllll}3 & 1158 & 29.86 & 19 & 53.46 & 155 & 15.10 \\ 3 & 2326 & 48.38 & 19 & 29.07 & 155 & 51.22\end{array}$

$4 \quad 221 \quad 5.05 \quad 1920.79 \quad 15512.36$ $434246.891921 .77 \quad 15512.64$

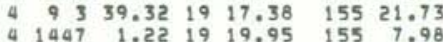
$5.232 \quad 24.42 \quad 1920.90 \quad 15512.98$

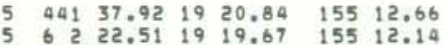
5101
6 $11.811927 .49 \quad 15550.95$ 610523.52 19 28.19 155 8.30 $\begin{array}{lllllll}6 & 1426 & 25.30 & 19 & 20.17 & 155 & 11.65\end{array}$

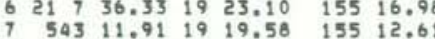

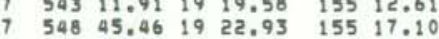

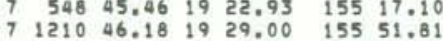

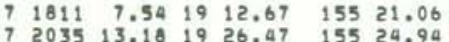
$7203513.181926 .47 \quad 15524.96$ 8 8 $05617.021920 .17 \quad 15513.36$ $\begin{array}{ll}5.83 & 31.33 \quad 19 \quad 23.61 \quad 155 \quad 15.5\end{array}$

$\begin{array}{rrrrrrr}8 & 827 & 1.53 & 19 & 20.77 & 155 & 7.68 \\ 8 & 1139 & 48.15 & 19 & 25.71 & 155 & 50.56\end{array}$

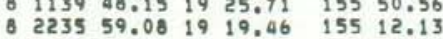
$8223945.26 \quad 1920.98 \quad 155 \quad 3.44$ 925014.83 $\begin{array}{lllllll}9 & 140 & 36.66 & 19 & 23.12 & 155 & 14.83 \\ 9 & 212 & 35.91 & 19 & 22.96 & 155 & 14.75\end{array}$ $217 \quad 27.74$ 19 23.19 $155 \quad 17.08$ $\begin{array}{lllllllllllll}7.95 & 2.1 & 2.1 & 33 & 0 & 72 & .15 & 5 & .5 & .6 & 27 & \text { UER }\end{array}$

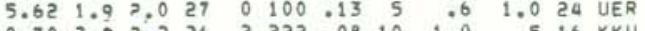

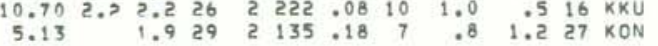

$\begin{array}{lllllllllll}8.45 & 1 . A & 1.9 & 26 & 0 & 74 & .11 & 4 & .5 & .6 & 22 \\ 0.96 R\end{array}$

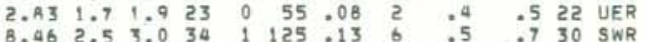

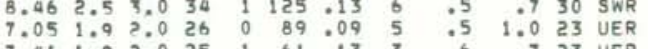

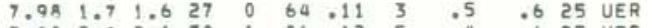

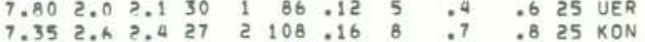

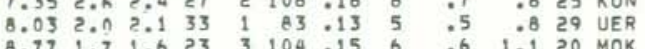

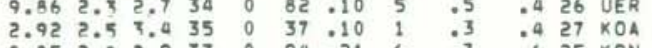

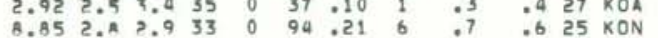

$\begin{array}{lllllllllllll}7.87 & 2.7 & 3.2 & 28 & 2 & 164 & 08 & 11 & 1.0 & 1.7 & 19 & \text { LSW L } & \end{array}$

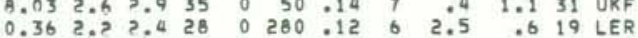

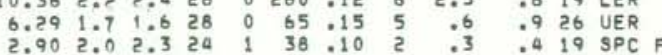

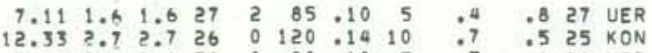

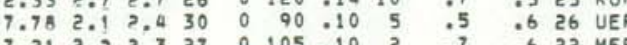

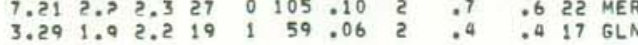
$\begin{array}{llllllllllllll}2.86 & 1.5 & .9 & 16 & 0 & 65 & .08 & 2 & .4 & .5 & 11 & \text { GLN } \\ 3.15 & 5 & 0.9 & 16 & 0 & 66 & .08 & 2 & .4 & .5 & 12 & \text { UER }\end{array}$ $\begin{array}{llllllllllll}3.15 & 1.5 & .9 & 16 & 0 & 66 & 0.08 & 2 & .4 & .5 & 11 & \text { OLN } \\ 2.87 & 2.5 & 35 & 1 & 36 & .10 & 0 & .3 & .3 & 31 & \text { SPC }\end{array}$

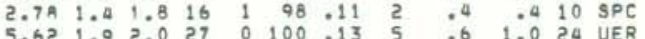
$\begin{array}{llllllllllll}8.73 & 2.3 & 2.4 & 30 & 0 & 81 & .09 & 5 & .4 & .7 & 27 & \text { UER } \\ 3.00 & 2.7 & 2.8 & 29 & 0 & 48 & .10 & 1 & .3 & .3 & 25 & \text { SPC }\end{array}$ 


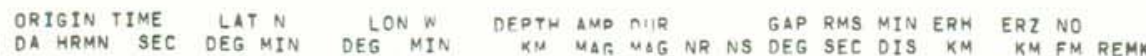

$\begin{array}{lllllllll}1978 \text { OCT } 9 & 6 & 0 & 55.88 & 19 & 20.57 & 155 & 10.20\end{array}$

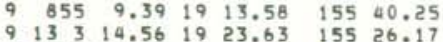
$134011.56 \quad 1923.65$
0

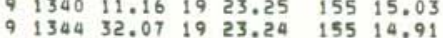

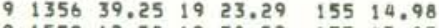

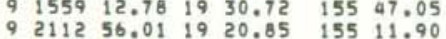
$9225725.071921 .51 \quad 155 \quad-11$

$10 \quad 527 \quad 12.55 \quad 19 \quad 19.93 \quad 155 \quad 11.75$

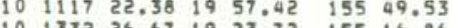

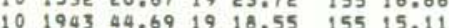
$10 \quad 2157 \quad 23.15 \quad 19 \quad 18.45 \quad 155 \quad 14.85$

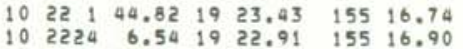

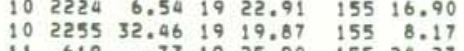

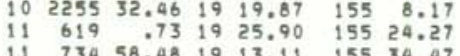
$11 \quad 1049 \quad 36.77 \quad 1921.03 \quad 155 \quad 12.92$

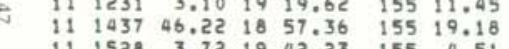

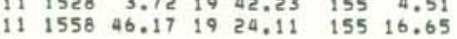
$\begin{array}{lrlllll}11 & 1837 & 11.36 & 19 & 21.06 & 155 & 6.13\end{array}$ $\begin{array}{rrrrrrr}12 & 351 & 58.58 & 19 & 22.81 & 155 & 15.22 \\ 12 & 1530 & 34.01 & 19 & 24.08 & 155 & 25.09\end{array}$

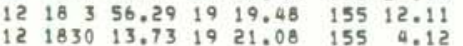
$\begin{array}{lllllll}12 & 2227 \quad 36.90 \quad 19 & 20.56 \quad 155 & 13.13\end{array}$

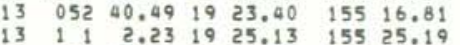

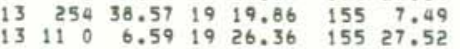
$\begin{array}{lllllll}13 & 1140 & 5.54 & 19 & 12.63 & 155 & 33.63\end{array}$ $\begin{array}{lllllll}13 & 1155 & 2.48 & 19 & 7.74 & 155 & 26.01\end{array}$ $\begin{array}{lllllll}13 & 123 & 18.62 & 19 & 11.86 & 156 & 39.02 \\ 13 & 1213 & 17.85 & 19 & 12.09 & 156 & 38.98\end{array}$

$\begin{array}{lllllll}13 & 1230 & 17.56 & 19 & 13.54 & 156 & 39.01\end{array}$ $13125016.091915 .43 \quad 15639.2$ $\begin{array}{lllllll}13 & 13 & 21.62 & 19 & 14.97 & 156 & 18.74 \\ 13 & 1310 & 18.84 & 19 & 15.44 & 156 & 20.29\end{array}$

$\begin{array}{llllllll}13 & 1320 & 20.56 & 19 & 16.92 \quad 156 & 14.22\end{array}$ $13 \quad 133022.1319 \quad 18.72 \quad 15612.91$

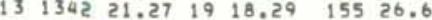

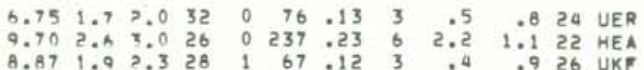

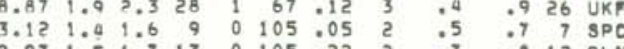
$\begin{array}{llllllllllll}3.31 & 1.4 & 1.4 & 10 & 0 & 103 & .02 & 2 & .4 & .5 & 10 & \text { GLN }\end{array}$

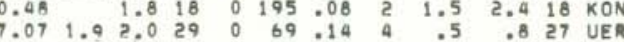

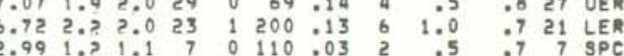

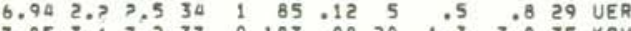

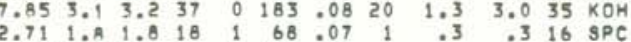

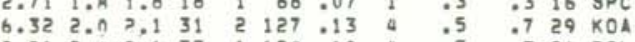
$8.812 .42 .6 \quad 33 \quad 1124.10$ 4 50.526 POL

$\begin{array}{llllllllllll}2.91 & 1.7 & 2.3 & 23 & 1 & 66 & .10 & 0 & .3 & .4 & 19 & \text { SPC } \\ 2.82 & 2.0 & 2.6 & 24 & 2 & 47 & .09 & 1 & .3 & .3 & 22 & K O A\end{array}$

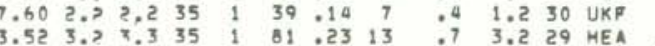

$\begin{array}{llllllllllll}8.25 & 2.1 & 2.4 & 32 & 0 & 60 & 13 & 3 & .4 & .6 & 30 & \text { UER } \\ 7.05 & 2.0 & 0.2 & 28 & 0 & 93 & 0.10 & 5 & .5 & .8 & 26 & \text { UER }\end{array}$

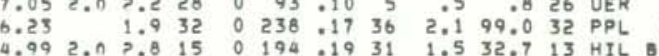

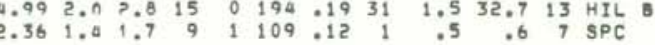
$\begin{array}{llllllllllll}8.43 & 2.6 & 2.8 & 34 & 1 & 95 & .11 & 6 & .5 & .6 & 30 & \text { UER } \\ 30.99 & & 1.7 & 30 & 0 & 51 & .07 & 1 & .7 & 1.2 & 27 & \text { DEP }\end{array}$ \begin{tabular}{llllllllllll}
0.99 & 1.9 & 2,1 & 28 & 1 & 76 & .09 & 5 & .4 & .7 & 26 & UKF \\
\hline
\end{tabular} $\begin{array}{llllllllllll}6.51 & 2.0 & 2.1 & 33 & 0 & 90 & .11 & 5 & .5 & .7 & 28 & \text { UER } \\ 6.36 & 2.2 & 2.4 & 34 & 2 & 89 & .12 & 3 & .5 & .9 & 27 & \text { MER }\end{array}$

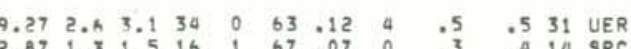
$\begin{array}{lllllllllllll}2.87 & 1.2 & 1.5 & 16 & 1 & 67 & .07 & 0 & .3 & .4 & 14 & \text { SPC } \\ 8.69 & 1.8 & 1.8 & 34 & 1 & 49 & .13 & 6 & .4 & 9 & 30 & \text { UKF }\end{array}$

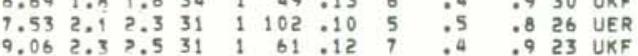

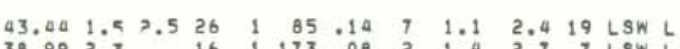

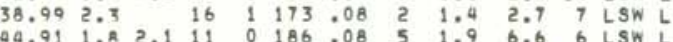

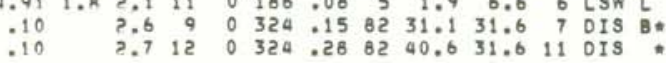
$\begin{array}{lllllllllllllll}10 & 3.2 & 7.2 & 13 & 0 & 317 & .17 & 82 & 21.6 & 31.6 & 13 & 019 & \text { B. }\end{array}$

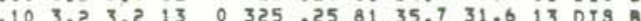

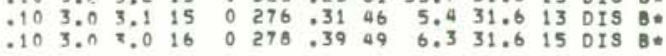

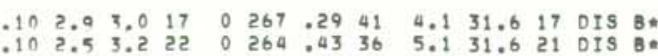

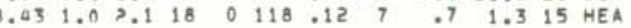

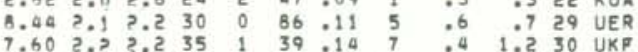

YEAR MON ORIGIN TIME LAT N

1978 OCT $13 \quad 1352 \quad 19.12 \quad 1923.26 \quad 156 \quad 3.61$

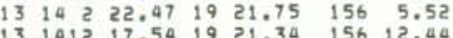
13 1422 19.421922 .70156 .43 $\begin{array}{rrrrrrr}13 & 1433 & 25.49 & 19 & 14.75 & 155 & 1.14 \\ 13 & 1442 & 18.06 & 19 & 23.45 & 155 & 56.68\end{array}$

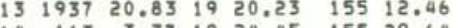
$14 \quad 113 \quad 3.37$ 19 24.05 155 29.64

$\begin{array}{lllllll}14 & 333 & 44.90 & 19 & 20.71 & 155 & 12.01 \\ 14 & 534 & 32.19 & 19 & 23.00 & 155 & 16.89\end{array}$ $14 \quad 534 \quad 32.19 \quad 1923.00 \quad 155 \quad 16.89$

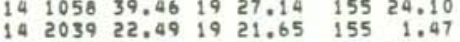
$15033 \quad 20.60 \quad 1923.75 \quad 15459.27$

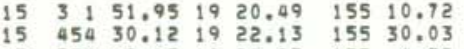
$15 \quad 535 \quad 48.65 \quad 1922.93 \quad 155 \quad 4.32$ 15 1411 $24.011929,70 \quad 15514.32$ $\begin{array}{rrrrrrr}15 & 2127 & 54.95 & 19 & 19.76 & 155 & 11.65 \\ 16 & 024 & .62 & 19 & 20.87 & 155 & 2.81\end{array}$

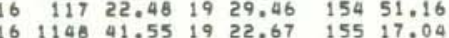

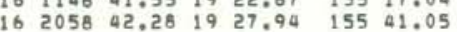
$\begin{array}{lllllll}16 & 2219 & 5.36 & 19 & 22.54 & 155 & 17.07\end{array}$ $17 \quad 23548.14 \quad 19 \quad 19.93 \quad 155 \quad 8.05$

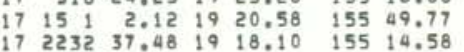
$\begin{array}{lllllll}17 & 2242 & 44.65 & 19 & 19.89 & 155 & 7.80\end{array}$

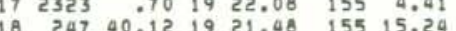
18 $540 \quad 37.91 \quad 19 \quad 35.13 \quad 155 \quad 50.28$ $18 \quad 542 \quad 7.0819 \quad 35.44 \quad 155 \quad 52.29$ $\begin{array}{lllllll}18 & 546 & 37.00 & 19 & 20.02 & 155 & 10.63\end{array}$ $1861655.551921 .32 \quad 15515.35$

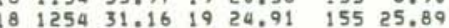

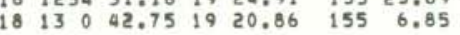
$\begin{array}{lllllll}18 & 2012 & 35.90 & 19 & 20.88 & 155 & 2.44 \\ 18 & 2037 & 11.79 & 19 & 25.25 & 155 & 27.28\end{array}$ $\begin{array}{lllllll}19 & 040 & 5.47 & 19 & 20.50 \quad 155 & 2.44\end{array}$ $\begin{array}{lllllll}19 & 746 & 29.72 & 19 & 23.77 & 155 & 16.71 \\ 19 & 828 & 32.03 & 19 & 16.73 & 155 & 21.28\end{array}$ $\begin{array}{lllllll}19 & 1147 & 27.37 & 19 & 20.27 & 155 & 13.09\end{array}$

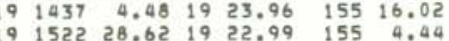

DEPTH AMP RIR GAP RMS MIN ERH ERZ NO

$\begin{array}{llllllllllllllll}.10 & 7.9 & 7.7 & 23 & 0 & 255 & .30 & 19 & 3.3 & 31.6 & 22 & \text { KON R. }\end{array}$

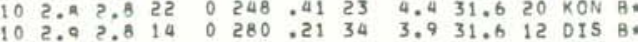

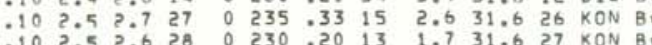

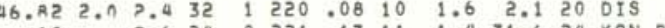

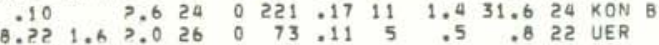

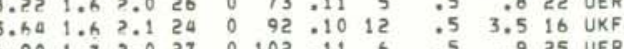
.5 .819 UER

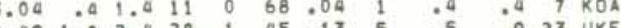

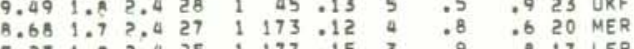
$5.23 \quad 1.90 .425 \quad 1177, .15$ $\begin{array}{rrrrrrrrrrrr}8.38 & 2.2 & 2.6 & 28 & 1 & 77 & .10 & 3 & .4 & .7 & 25 & \text { UER } \\ 7.05 & 1.4 & 2.3 & 26 & 0 & 112 & 112 & 4 & .6 & 1.0 & 22 & \text { MOK }\end{array}$

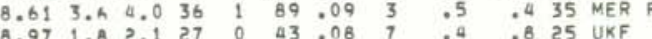

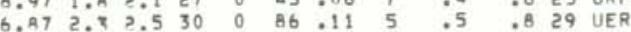
$\begin{array}{lllllllllll}7.50 & 2.2 & 2.8 & 30 & 1 & 89 & .11 & 5 & .4 & .7 & 28 \\ 7.54 & \text { UER }\end{array}$ 7.

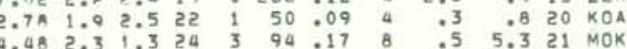
$\begin{array}{llllllllllll}3.00 & 1.7 & 2.4 & 20 & 0 & 51.08 & 4 & .4 & .9 & 16 & \text { KOA }\end{array}$

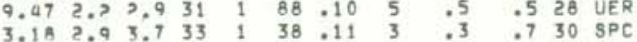

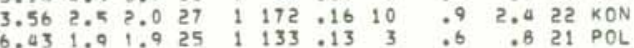
$\begin{array}{llllllllllllll}9.57 & 3.2 & 3.7 & 36 & 1 & 95 & .11 & 5 & .6 & .4 & 34 & \text { UER }\end{array}$

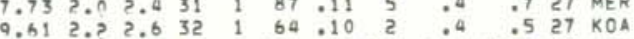

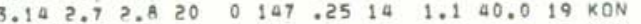
$\begin{array}{lllllllllllll}6.09 & 2.2 & 2.4 & 17 & 0 & 215 & .17 & 12 & 1.1 & 1.1 & 13 & \mathrm{KON}\end{array}$

$7.971 .42 .330 \quad 1 \quad 86.09$ a $\quad .4 \quad .624$ UER F

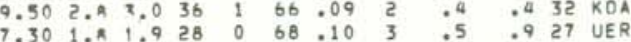

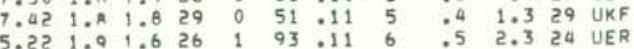

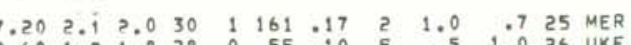
$9.21,01020 \quad 1 \quad 175.16 \quad 1 \quad 1.0 \quad 1.217$ MER

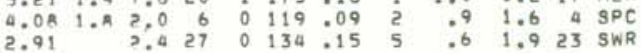
\begin{tabular}{llllllllllll}
1.38 & 1.9 & 2.1 & 29 & 0 & 67 & .12 & 4 & .5 & .7 & 29 \\
\hline & $.7 E R$
\end{tabular}

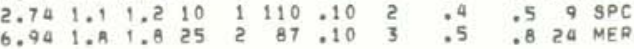




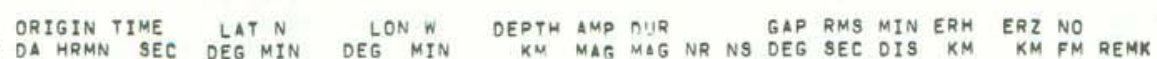

1978 OCT $19163255.721921 .45 \quad 155 \quad 6.22$

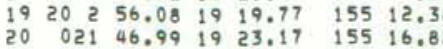
$20 \quad 21554.82 \quad 1932.43 \quad 15525.07$

$\begin{array}{lllllll}20 & 1150 & 56.71 & 19 & 17.38 & 155 & 30.62\end{array}$ $\begin{array}{lllllll}20 & 1254 & 2.20 & 19 & 17.13 & 155 & 28.29\end{array}$

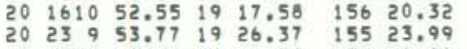
$20 \quad 2342 \quad 10.83 \quad 1926.30 \quad 15524.01$ $\begin{array}{rrrrrrr}21 & 655 & 49.82 & 19 & 21.37 & 155 & 10.60 \\ 21 & 1249 & 3.73 & 19 & 26.37 & 155 & 28.90\end{array}$ $22 \quad 241 \quad 53.58 \quad 19 \quad 16.40 \quad 155 \quad 23.47$

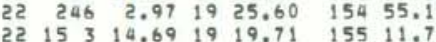

$\begin{array}{lllllll}22 & 1513 & 1.48 & 19 & 12.47 & 155 & 19.93\end{array}$ $\begin{array}{lllllll}22 & 2347 & 6.28 & 19 & 19.63 & 155 & 11.52 \\ 23 & 8 & 39.15 & 19 & 20.17 & 155 & 12.77\end{array}$

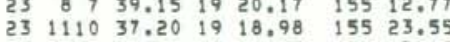
$23133911.941921 .47 \quad 155 \quad 7.13$

$\begin{array}{lllrllll}23 & 1534 & 3.30 & 19 & 19.47 & 155 & 11.79 \\ 23 & 1913 & 22.69 & 19 & 20.50 & 155 & 12.49\end{array}$

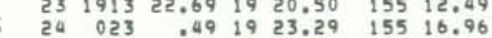
$\begin{array}{rrrrrrr}24 & 334 & 24.35 & 19 & 23.07 & 155 & 2.15 \\ 24 & 1433 & 13.99 & 19 & 19.47 & 155 & 12.04\end{array}$ $\begin{array}{lllllll}24 & 1527 \quad 51.40 \quad 19 & 20.01 \quad 155 & 11.20\end{array}$ $25 \quad 34856.33$ 19 $20.30 \quad 155 \quad 3.7$ $252053 \quad 37.791922 .13 \quad 155 \quad 6.01$

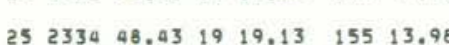
$26 \quad 228 \quad 33.51 \quad 1921.40 \quad 155 \quad 6.6$ $\begin{array}{lrrrrrr}26 & 318 & 58.82 & 19 & 21.96 & 155 & .16 \\ 26 & 349 & 5.26 & 19 & 20.15 & 155 & 6.80\end{array}$ $26 \quad 615 \quad 7.48 \quad 19 \quad 18.07 \quad 155 \quad 13.20$

$\begin{array}{lllllll}26 & 747 & 22.19 & 19 & 20.17 & 155 & 8.21\end{array}$ $27 \quad 424 \quad 47.76 \quad 19 \quad 39.50 \quad 155 \quad 15.78$ $\begin{array}{lllllll}27 & 552 & 36.67 & 19 & 10.92 & 155 & 13.48 \\ 28 & 552 & 53.73 & 19 & 20.25 & 155 & 11.44\end{array}$ $\begin{array}{lllllll}28 & 1057 & 34.73 & 19 & 18.98 & 155 & 11.30\end{array}$ $28 \quad 1450 \quad 47.11 \quad 1924.00 \quad 15516.11$

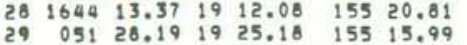
$29 \quad 328 \quad 54.89 \quad 1922.62 \quad 155 \quad 4.94$

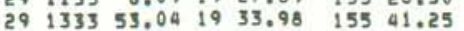

$\begin{array}{lllllllllll}7.22 & 2.2 & 2.3 & 32 & 2 & 86 & .12 & 6 & .5 & .9 & 29 \\ 0 & 0.25\end{array}$

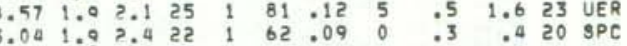

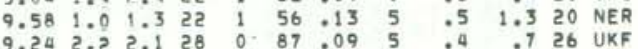

$\begin{array}{llllllllllll}1.23 & 2.1 & 1.9 & 25 & 0 & 115 & .18 & 11 & .6 & 16.0 & 21 & \text { HEA }\end{array}$

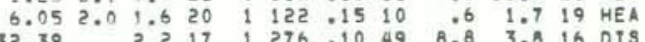

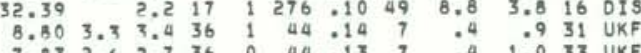

$\begin{array}{llllllllllll}8.31 & 2.0 & 2.1 & 30 & 0 & 64 & .11 & 2 & .4 & .7 & 29 & \text { UER }\end{array}$

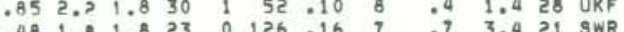

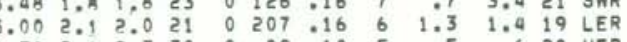
$\begin{array}{lllllllllllll}46.84 & 2.9 & 3.2 & 40 & 3 & 169 & .12 & 10 & .8 & 1.3 & 38 & \text { HLP } & \end{array}$

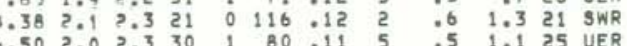
$\begin{array}{lllllllllll}8.87 & 2.6 & 2.9 & 32 & 0 & 94 & .10 & 5 & .5 & .5 & 29 \\ 7.38 & \text { UER }\end{array}$

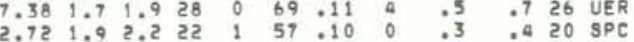

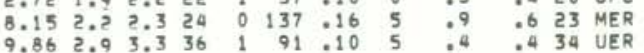
$7.87 \quad 1.91 .934 \quad 1 \quad 86.11 \quad 4 \quad .4 \quad .729$ UER

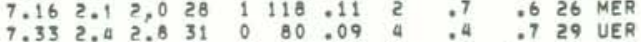

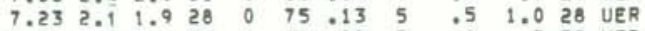

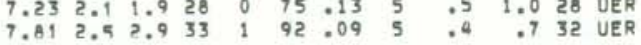
$\begin{array}{rlllllllllll}7.67 & 1.2 & 1.9 & 27 & 0 & 68 & .12 & 4 & .5 & .7 & 25 & \text { UER } \\ 7.75 & 2.1 & 2.4 & 30 & 0 & 85 & .09 & 6 & 5 & .7 & 28 & \text { UER }\end{array}$

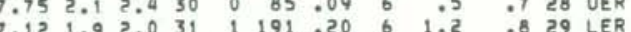

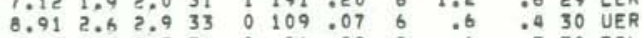
$8.952 .25 .531 \quad 0 \quad 96.09 \quad 2 \quad .6 \quad .530$ POL

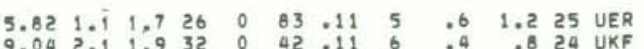

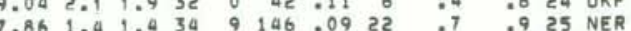

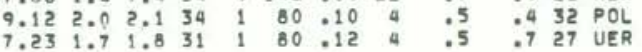
$\begin{array}{llllllllllllll}9.29 & 2.0 & 2.3 & 30 & 0 & 110 & .11 & 5 & .6 & .6 & 26 & \text { POL }\end{array}$

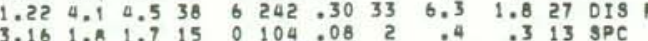

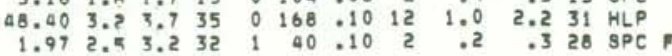

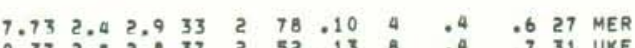
56 1.4 1.518 1 $127.1410 \quad .85,315 \mathrm{MOK}$

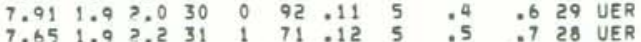

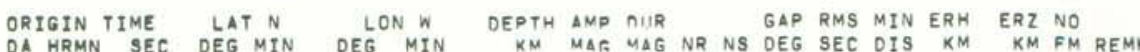

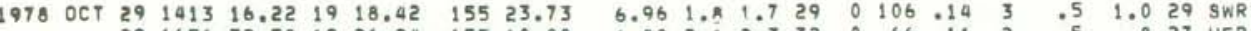

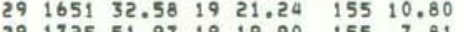

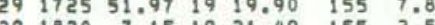
30 (850 11.9919 20.43 155 2.56

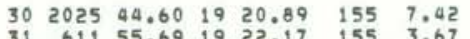

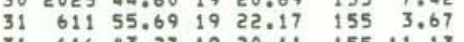

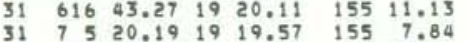
$31 \quad 81542.041924 .08 \quad 15526.86$

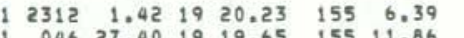

45757.83 i9 $19.65 \quad 15511.86$

$5623.611919 .75 \quad 15511.48$

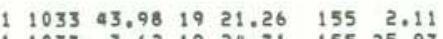
$1037 \quad 7.621924 .71 \quad 15525.97$ $1833 \quad 45.21 \quad 1924.16 \quad 15456.61$

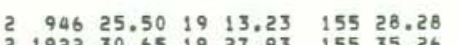
$192230.651927 .93 \quad 15535.26$ ? $221211.991920 .81 \quad 15510.76$ $2225032.51 \quad 1934.61 \quad 15555.61$

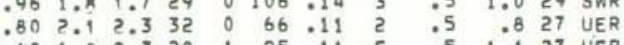

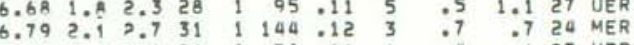

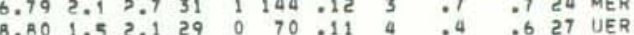

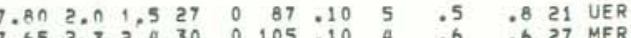

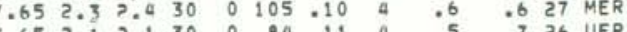

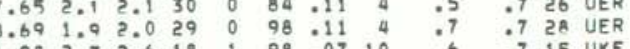

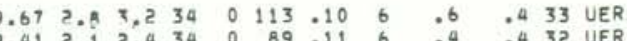

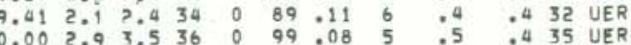

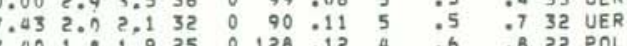

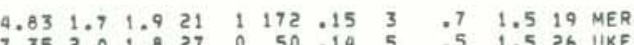

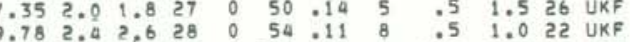

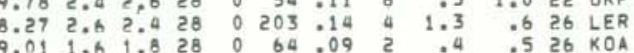

$\begin{array}{lrlllll}2 & 2358 & 36.50 & 19 & 21.24 & 155 & 6.11 \\ 3 & 346 & 53.85 & 19 & 27.69 & 155 & 34.81\end{array}$ $34,93.66$ 19 $45.42 \quad 15454.13$

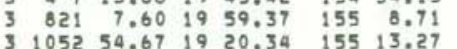
$6.402,52.324 \quad 0 \quad 96.20$

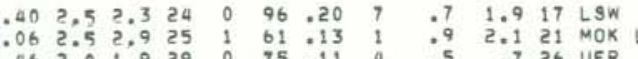

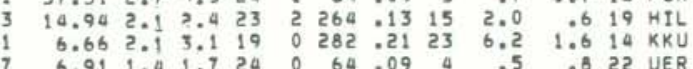

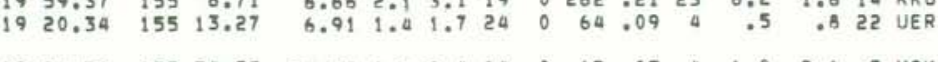

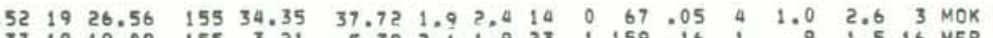

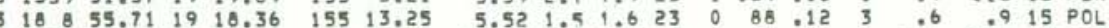

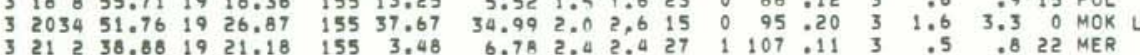

$\begin{array}{llllllllllllllllll}3 & 2348 & 7.40 & 19 & 15.75 & 155 & 24.82 & 34.97 & 1.9 & 2.0 & 33 & 3 & 121 & .09 & 9 & .7 & .8 & 25 \text { LSW }\end{array}$

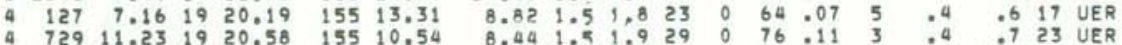

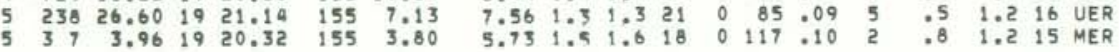

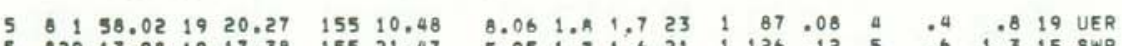

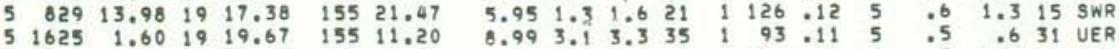

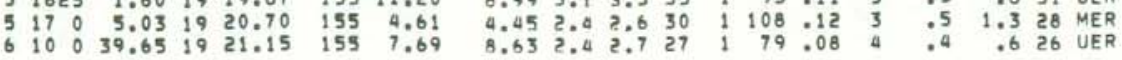
$\begin{array}{lllllllllllllllll}62031 & 6.46 & 19 & 20.38 & 155 & 10.64 & 13.50 & 2.4 & 2.9 & 19 & 0 & 166 & .49 & 3 & 2.4 & 1.3 & 17 \text { UER }\end{array}$ $6048 \quad 16.66$
6 


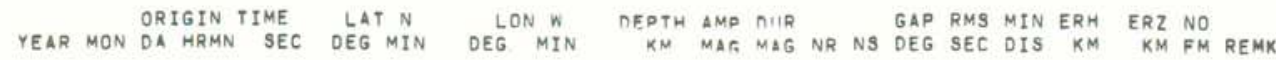
1978 NOV $7 \begin{array}{llllllllllllllllll}1256 & 57.79 & 19 & 48.04 & 156 & 8.42 & 12.35 & 3.0 & 7.9 & 17 & 1 & 200 & .09 & 34 & 1.7 & .9 & 15 \text { KON }\end{array}$

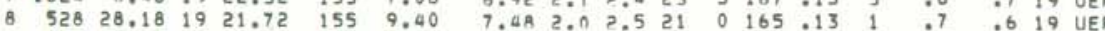

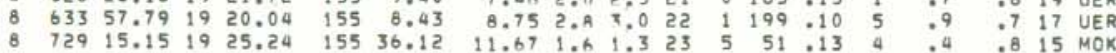
$\begin{array}{lllllllllllllllllll}8 & 1314 & 10.94 & 19 & 42.89 & 155 & 41.95 & 14.10 & 2 . \times & 2.5 & 15 & 0 & 163 & .13 & 15 & 1.0 & .8 & 15 \mathrm{KKV}\end{array}$

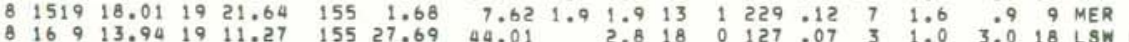

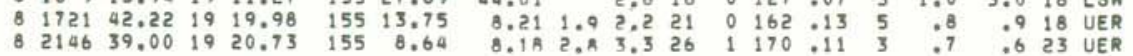
$\begin{array}{lllllllllllllllllll}8 & 2216 & 17.57 & 19 & 17.43 & 155 & 21.51 & 7.46 & 2.0 & 2.1 & 19 & 1 & 151 & .13 & 6 & .6 & 1.4 & 15 & \text { SWR }\end{array}$

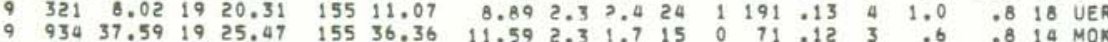

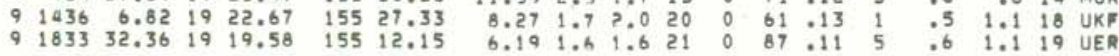
$10 \quad 148 \quad 49.55 \quad 19 \quad 25.28 \quad 155 \quad 24.48$ $10 \quad 533 \quad 7.16$ $1017226.811920 .72 \quad 15512.84$

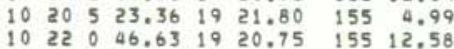
$10 \quad 2344 \quad 38.74 \quad 19 \quad 19.55 \quad 155 \quad 15.99$

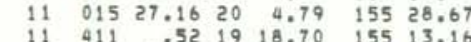
i1 $629 \quad 4.791920 .18 \quad 155 \quad 11.8$

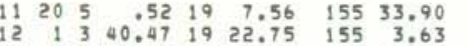
$125047.981920 .86 \quad 15511.53$ 12528 (12.20 19.80015537 .9

$12 \quad 946 \quad 38.69 \quad 19 \quad 23.92 \quad 155 \quad 25.34$ $12123954.401922 .04 \quad 155 \quad 2.68$ 12145851.071935 .31 155 59.63

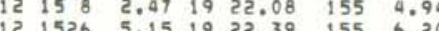
$\begin{array}{lllllll}12 & 1718 & .18 & 19 & 19.75 & 155 & 11.57 \\ 12 & 2158 & 25.80 & 19 & 23.03 & 155 & 2.19\end{array}$

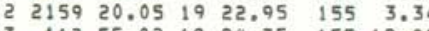

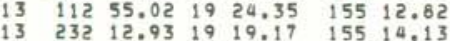

$\begin{array}{rrrrrrr}13 & 959 & 3.42 & 19 & 20.05 & 155 & 8.35 \\ 13 & 1829 & 51.60 & 19 & 8.23 & 155 & 26.13\end{array}$ $132212 \quad 8.181921 .44 \quad 15459.68$ $14 \quad 2033 \quad 12.1320 \quad 12.96 \quad 156 \quad 8.12$ $\begin{array}{lllllll}15 & 337 & 17.74 & 19 & 25.43 & 155 & 24.31 \\ 15 & 741 & 24.34 & 99 & 11.00 & 155 & 40.62\end{array}$

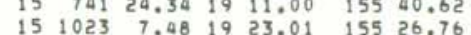

$\begin{array}{llllllllllll}\text { B. } 20 & 2.1 & 2.1 & 23 & 0 & 60 & .11 & 8 & .4 & 1.2 & 23 & \text { UKF }\end{array}$ $7.051 .41 .820 \quad 0 \quad 64.13 \quad 4 \quad .6 \quad 2.513$ MOK

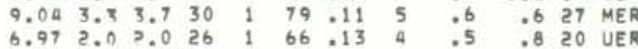
$\begin{array}{llllllllllll}31.93 & 2.4 & 2.9 & 26 & 0 & 92 & .10 & 5 & .7 & 1.4 & 25 & \text { DEP }\end{array}$

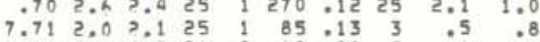

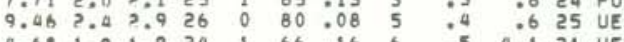

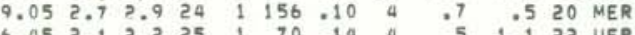

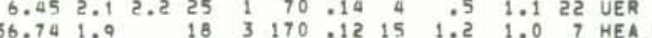

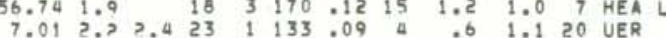

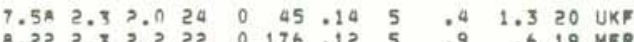

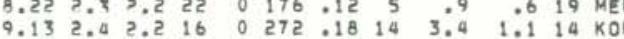

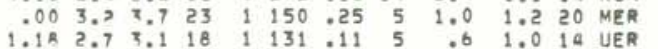

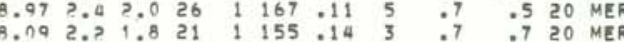

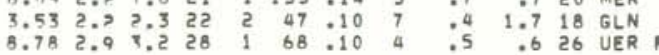

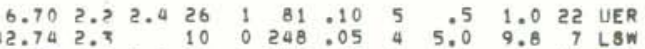

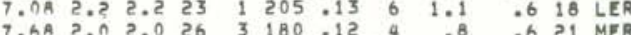

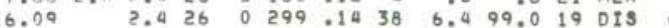

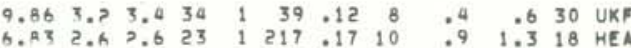

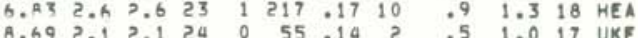
$\begin{array}{llllllllllllllll}42.07 & 2.3 & 2.4 & 15 & 0 & 143 & 117 & 11 & 1.6 & 4.3 & 3 & \text { LSW L }\end{array}$ $\begin{array}{lllllllllll}5.59 & 2.0 & 2.2 & 24 & 1 & 89 & .11 & 5 & .5 & 1.1 & 22 \\ 8 & \text { UER }\end{array}$
ORIGIN TIME LEAR MON DA HRMN SEC DEG MIN DEG MIN OEPTH AMP OIIR NR GAP RMS MIN ERH ERZ NO
KM MAF MAG NR NS DEG SEC DIS KM KM FM REMK 1978 NOV $15 \quad 1053 \quad 18.98 \quad 19 \quad 8.40 \quad 155 \quad 36.33$

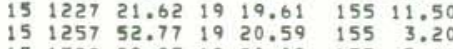
15132929.951920 .09 155 7.44 $\begin{array}{llllll}15 & 16 & 0 & 27.28 & 19 & 21.49 \quad 155 \quad 5.90\end{array}$

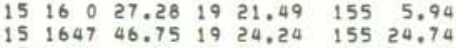

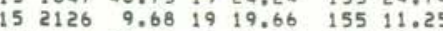

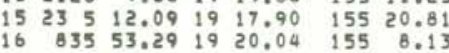

$\begin{array}{lllllll}16 & 1121 & 2.65 & 19 & 18.46 \quad 155 \quad 14.02\end{array}$ 16 1253 39.93 19 $18.03 \quad 155 \quad 12.89$

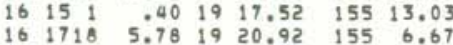
$\begin{array}{llllllll}16 & 2258 & 40.96 & 19 & 22.55 & 155 & 4.53\end{array}$ $7.07 \quad 155 \quad 6.87$
73.01

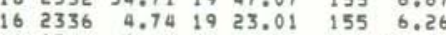

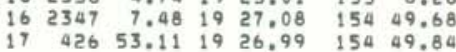

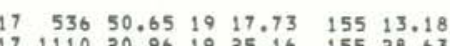
$7152948.741940 .87 \quad 155$. 2.40 $71621 \quad 34.36 \quad 1919.11 \quad 15511.99$
17

$\begin{array}{llllll}17 & 1714 & 15.24 & 19 & 54.90 \quad 155 & 14.35\end{array}$ $\begin{array}{lllllll}17 & 1951 & 36.57 & 19 & 21.77 & 155 & 6.56\end{array}$ $172043 \quad 37.08 \quad 19 \begin{array}{llll}14.15 & 155 & 36.66\end{array}$

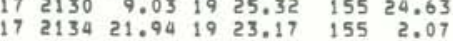

$\begin{array}{lllllll}17 & 2149 & 1.43 & 19 & 21.96 & 155 & 4.21\end{array}$

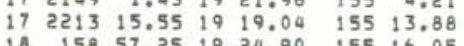
$\begin{array}{lllllll}18 & 158 & 57.25 & 19 & 24.90 & 155 & 16.05\end{array}$ $\begin{array}{lllllll}18 & 211 & 21.73 & 19 & 22.47 & 155 & 4.51 \\ 18 & 659 & 39.53 & 19 & 25.25 & 155 & 28.71\end{array}$

$\begin{array}{lllllll}18 & 1227 & 3.71 & 19 & 23.18 & 155 & 26.41\end{array}$ $\begin{array}{llllll}18 & 1233 & 15.75 & 19 & 23.18 & 155 \\ 18 & 26.41 & 155 & 4.41\end{array}$

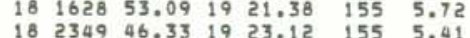
$\begin{array}{rrrrrrr}18 & 2349 & 46.33 & 19 & 23.12 & 155 & 5.41 \\ 19 & 149 & 56.38 & 19 & 22.56 & 155 & 2.58\end{array}$ $\begin{array}{lllllll}19 & 835 & 20.17 & 19 & 20.04 & 155 & 5.14\end{array}$ $\begin{array}{lllllll}19 & 1259 & 22.53 & 19 & 19.87 & 155 & 9.77 \\ 19 & 1341 & 25.50 & 10 & 20.85 & 155 & 13.26\end{array}$

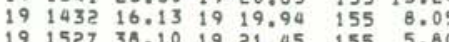

$\begin{array}{lllllll}19 & 1630 & 53.69 & 19 & 25.89 & 155 & 24.39 \\ 19 & 1720 & 21.63 & 19 & 23.93 & 155 & 26.12\end{array}$ $191741 \quad 49.471923 .93 \quad 15526.12$

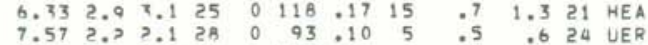

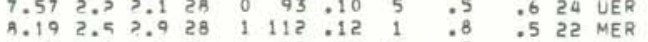

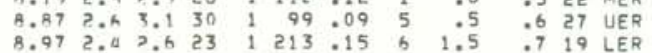
$\begin{array}{lllllllllll}6.27 & 1.4 & 1.6 & 25 & 1 & 87 & 112 & 6 & .5 & 1.2 & 22 \\ 1.3 E R\end{array}$

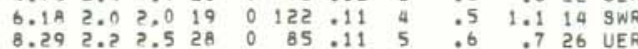
\begin{tabular}{lllllllllll}
6.44 & 1.5 & 1.6 & 28 & 2 & 77 & .12 & 3 & .5 & .9 & 27 \\
\hline
\end{tabular} 6.66 5.

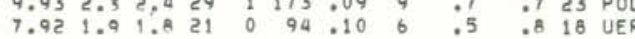
$\begin{array}{llllllllllll}9.20 & 2.0 & 1.7 & 25 & 0 & 86 & .10 & 4 & .7 & .7 & 19 & \text { MER }\end{array}$

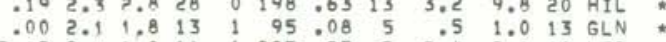

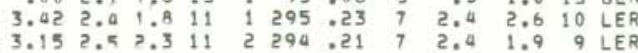
$\begin{array}{llllllllllll}7.83 & 2.1 & 2.1 & 29 & 0 & 107 & .13 & 1 & .5 & .7 & 28 & \text { POL }\end{array}$

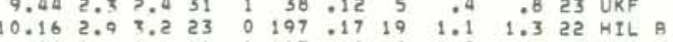

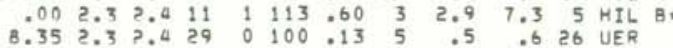

$\begin{array}{llllllllllll}38.04 & 2.5 & 2.1 & 36 & 6 & 241 & .11 & 11 & .7 & .6 & 33 & \mathrm{kKV}\end{array}$

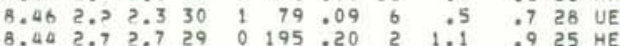

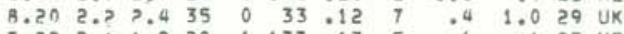

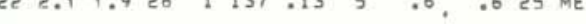
$\begin{array}{lllllllllll}7.89 & 2.4 & 2.7 & 30 & 0 & 91 & .10 & 4 & .5 & .6 & 25 \\ 7 E R\end{array}$

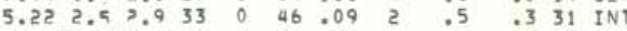

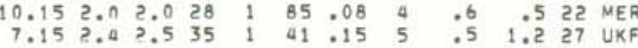

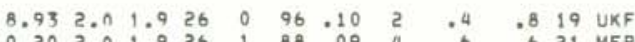

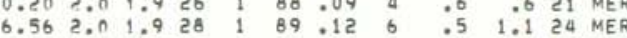

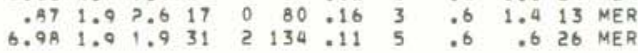

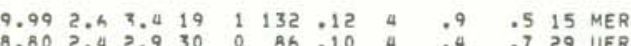
$9.032 .52 .930 \quad 86.10 \quad 4$

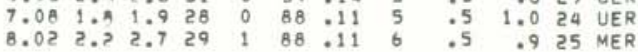

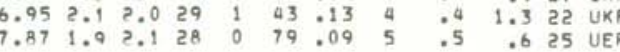

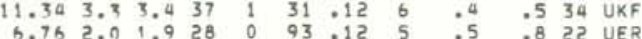

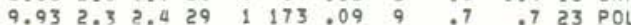

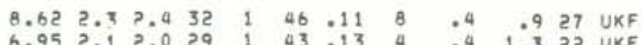


ORIGIN TIME LAT N LON W DEPTH AMP RUR GAP RMS MIN ERH ERZ NO 1978 NOV $191848 \quad 37.13 \quad 1920.03 \quad 155 \quad 11.55$

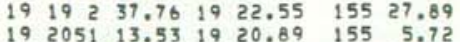
$19234917.931921 .40 \quad 15513.13$

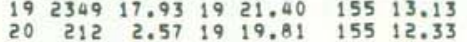

$\begin{array}{lrrrrrr}20 & 1351 & 4.85 & 19 & 19.84 & 155 & 11.70 \\ 20 & 16 & 20.92 & 19 & 21.23 & 155 & 6.03\end{array}$

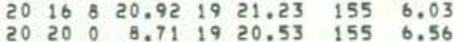
$202014 \quad 22.961919 .86 \quad 155 \quad 9.78$ $21 \quad 337 \quad 55.91$ iी $19.05 \quad 155$ 11.48

$\begin{array}{lrrrrrr}21 & 910 & 45.03 & 19 & 20.25 & 155 & 11.59 \\ 21 & 1210 & 30.01 & 19 & 17.37 & 155 & 13.13\end{array}$

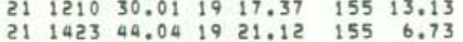
$\begin{array}{rrrrrrr}21 & 1423 & 44.04 & 19 & 21.12 & 155 & 6.73 \\ 22 & 216 & 36.21 & 19 & 21.77 & 155 & 14.97\end{array}$

$\begin{array}{lllllll}22 & 820 & 42.87 & 19 & 12.68 & 155 & 27.98\end{array}$

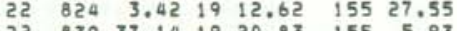
$22 \quad 83957.141920 .83$ 155 5.93

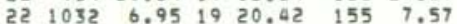
$22 \quad 104124.091912 .41 \quad 155 \quad 27.56$

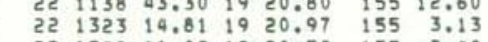

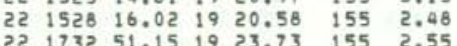
$\begin{array}{llllllll}22 & 1757 & 46.83 & 19 & 19.78 & 155 & 12.37\end{array}$ $\begin{array}{lllllll}23 & 117 & 36.15 & 19 & 21.90 & 155 & 6.67\end{array}$

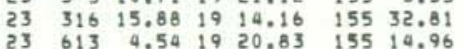
$\begin{array}{lllllll}23 & 754 & 40.55 & 19 & 20.82 & 155 & 2.27\end{array}$

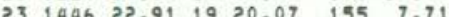
$23232539.74 \quad 1923.53 \quad 15528.53$

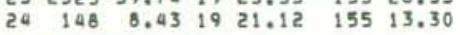

$\begin{array}{llllllll}24 & 3 & 5 & 42.32 & 19 & 25.65 & 154 & 57.27\end{array}$ $24 \quad 852 \quad 19.66 \quad 19 \quad 22.20 \quad 155 \quad 1.53$

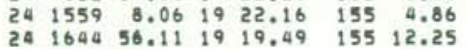
$\begin{array}{lllllll}24 & 2347 & 44.40 & 19 & 19.62 & 155 & 11.15\end{array}$

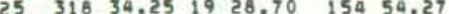
$\begin{array}{lllllll}25 & 558 & 44.88 & 19 & 19.76 & 155 & 17.51 \\ 25 & 925 & 38.32 & 19 & 20.30 & 155 & 8.61\end{array}$

$\begin{array}{lllllll}25 & 10 & 2 & 8.94 & 19 & 20.07 \quad 155 \quad 8.40\end{array}$ $\begin{array}{lllllll}25 & 1257 & 42.64 & 19 & 12.67 & 155 & 27.46 \\ 25 & 1458 & 39.65 & 19 & 21.06 & 155 & 13\end{array}$ $\begin{array}{llllllllllll}8.58 & 1.7 & 1.8 & 30 & 0 & 84 & .09 & 5 & .4 & .5 & 29 & \text { UER } \\ 8.66 & 2.3 & 2.3 & 31 & 0 & 61 & .12 & 1 & .4 & .8 & 26 & \text { UKF } \\ 0.33 & 2.0 & ? .2 & 20 & 0 & .01 & .11 & 5 & .4 & .6 & 25 & \text { MER }\end{array}$

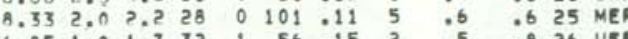
$\begin{array}{llllllllllll}6.85 & 1.2 & 1.7 & 32 & 1 & 56 & .15 & 2 & .5 & .8 & 26 & \text { UER } \\ 6.64 & 1.4 & 1.7 & 26 & 0 & 82 & .13 & 5 & .6 & 1.0 & 24 & \text { UER }\end{array}$

$\begin{array}{lllllllllllll}5.50 & 1.5 & 1.7 & 23 & 0 & 86 & .11 & 5 & .5 & 1.4 & 21 & \text { UER } \\ 6.19 & 2.2 & 1.8 & 24 & 1 & 92 & .10 & 6 & .5 & 1.2 & 22 & \text { UER } \\ 7.54 & 0.0 & .0 & 26 & 0 & 103 & .10 & 6 & .6 & .9 & 25 & \text { UER }\end{array}$

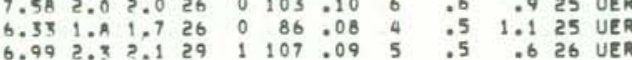
$\begin{array}{llllllllllll}7.35 & & 1.8 & 24 & 0 & 79 & 10 & 5 & .6 & .9 & 19 & \text { UER } \\ 8.02 & 1.4 & 1.9 & 27 & 0 & 134 & .12 & 1 & .7 & .8 & 22 & \text { POL }\end{array}$

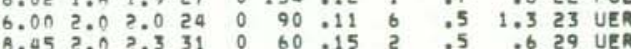

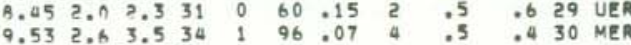
$\begin{array}{llllllllllll}5.24 & 3.1 & 3.4 & 31 & 0 & 101 & .15 & 6 & .6 & 1.6 & 28 & \text { LSW } \\ 6.68 & 2.4 & 2.7 & 30 & 0 & 111 & .17 & 6 & .6 & 1.3 & 21 & \text { LSW }\end{array}$

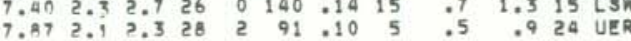

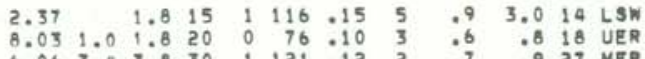

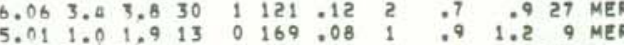

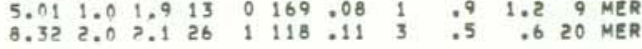
$\begin{array}{lllllllllllll}7.17 & . A & 1.3 & 23 & 0 & 81 & .10 & 5 & .6 & 1.0 & 17 & \text { UER }\end{array}$

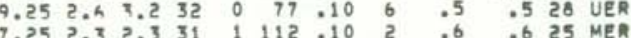

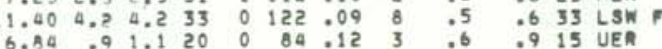
$\begin{array}{llllllllllll}4.61 & 1.92 & 2.1 & 20 & 0 & 170 & .15 & 2 & 1.1 & 1.5 & 13 & \text { MER }\end{array}$

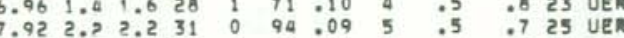

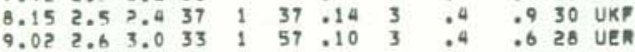
$\begin{array}{lllllllllllll}6.21 & 2.0 & 1.8 & 23 & 0 & 175 & .13 & 3 & .9 & .7 & 23 & \text { LER }\end{array}$

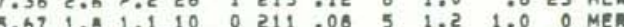

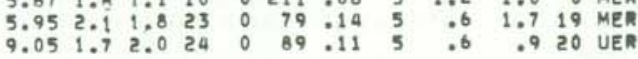

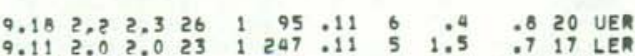
$2.98 \quad 2.0 \quad 2.4 \quad 17 \quad 1154 \quad .10 \quad 2 \quad 2.0 \quad 1.710$ LER

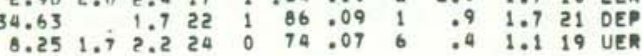

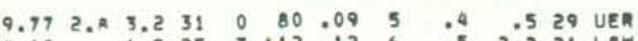

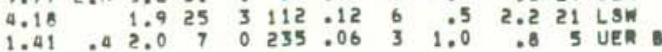

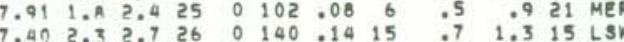

ORIGIN TIME
OA HRMN SAT N DEG MIN DEN W

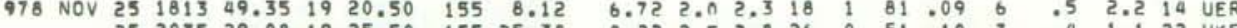

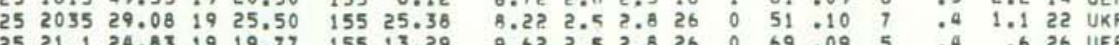

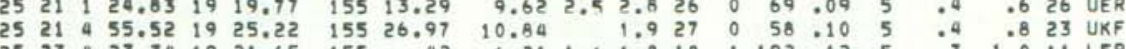

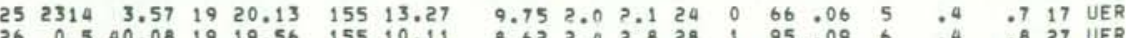
\begin{tabular}{llllllllll}
\hline & 27 & & 0
\end{tabular}

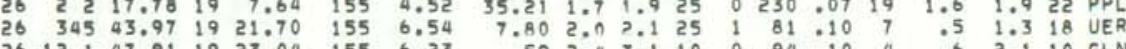

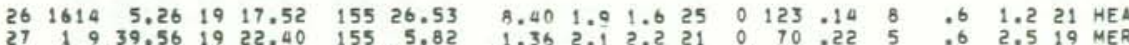

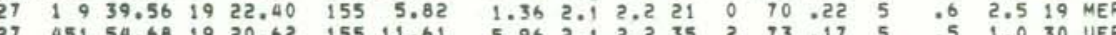

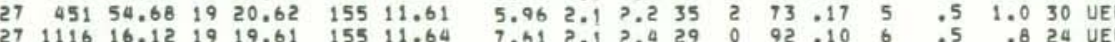

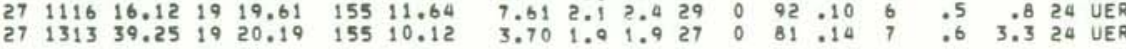

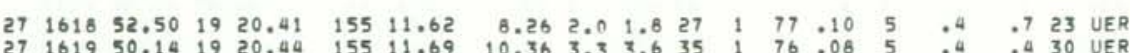

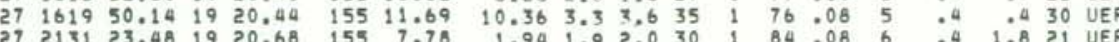

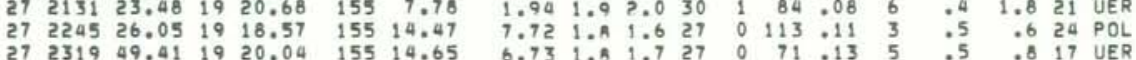
$28 \quad 129 \begin{array}{lllllllllllllllll}30.11 & 19 & 22.51 & 155 & 1.37 & 6.54 & 2.1 & 2.1 & 24 & 1 & 161 & .15 & 6 & .8 & 1.1 & 21 & \text { MER }\end{array}$

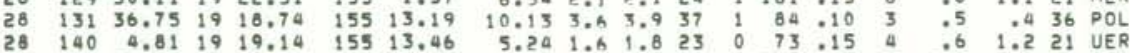

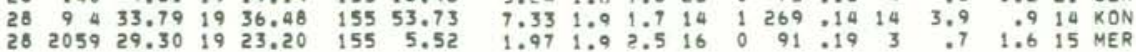

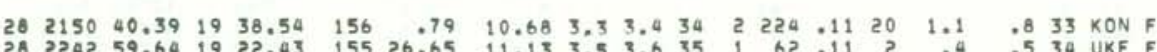

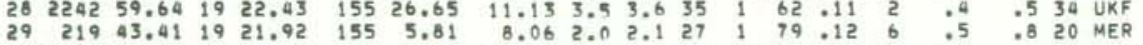

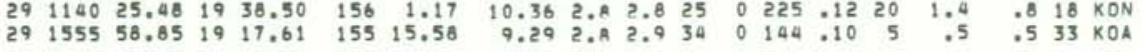

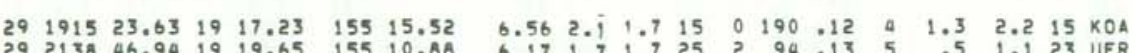

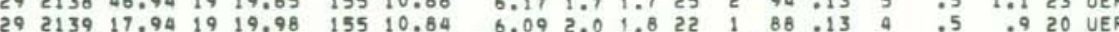

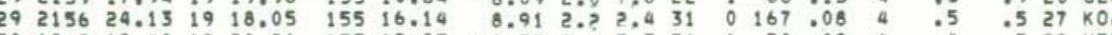

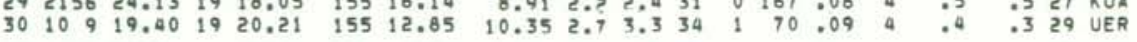

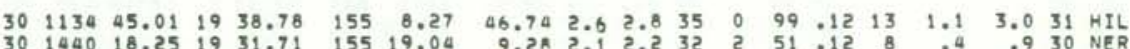

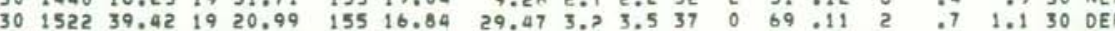

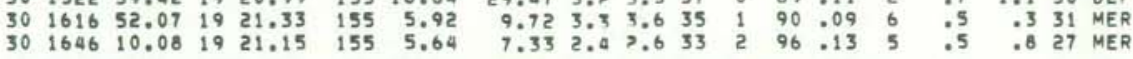

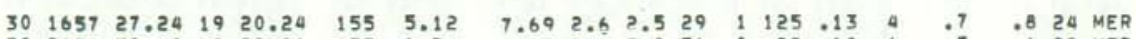

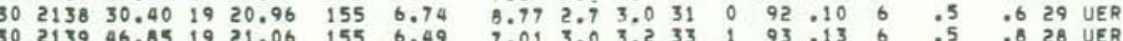

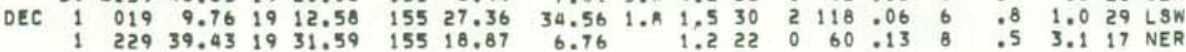

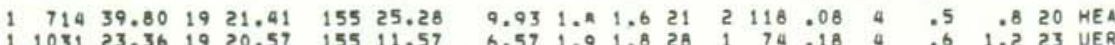

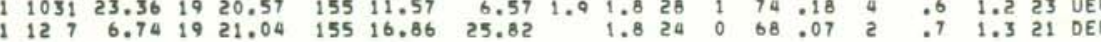




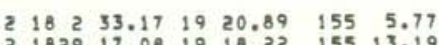

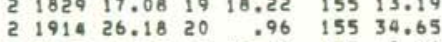

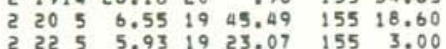

$\begin{array}{lllllll}3 & 121 & 21.23 & 19 & 20.78 \quad 155 & 11.44\end{array}$ $\begin{array}{lllllll}3 & 417 & 57.36 & 19 & 23.56 & 155 & 26.56 \\ 3 & 445 & 24.00 & 19 & 21.02 & 155 & 7.20\end{array}$ $\begin{array}{lllllll}3 & 1027 & 5.28 & 19 & 19.75 & 155 & 11.95 \\ 3 & 114 & 16.82 & 19 & 45.16 & 155 & 22.32\end{array}$

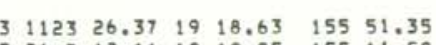

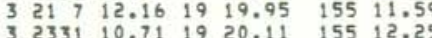
$\begin{array}{lllllll}4 & 311 & 10.35 & 19 & 19.75 & 155 & 7.86 \\ 4 & 339 & 31.24 & 19 & 21.11 & 155 & 3.31\end{array}$

$\begin{array}{lllllll}4 & 1256 & 36.96 & 19 & 20.66 & 155 & 7.61\end{array}$

$42152 \quad 24.61 \quad 1920.04 \quad 155 \quad 12.87$

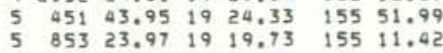

$\begin{array}{llllllll}5 & 857 & 33.79 & 19 & 19.51 & 155 & 11.38\end{array}$

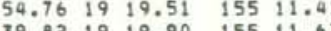

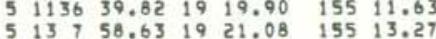
$5152111.771921 .57 \quad 155 \quad 1.72$

$\begin{array}{lllllll}5 & 1651 & .10 & 19 & 19.93 & 155 & 11.15 \\ 5 & 1742 & 23.58 & 19 & 20.01 & 155 & 50.89\end{array}$ $5 \quad 1745 \quad 41.211920 .03 \quad 15511.25$

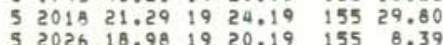

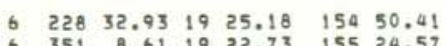

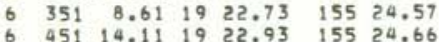
$\begin{array}{lrlllll}6 & 451 & 14.11 & 19 & 22.93 & 155 & 24.66 \\ 6 & 1311 & 28.00 & 19 & 20.17 & 155 & 11.23\end{array}$ $\begin{array}{ll}6 \quad 1633 \quad 43.52 \quad 19 & 17.37 \quad 155 \quad 22.02\end{array}$

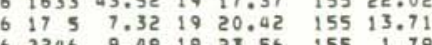

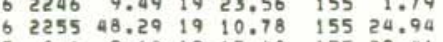

$\begin{array}{rrrrrrr}7 & 115 & 51.55 & 19 & 20.93 & 155 & 6.25 \\ 7 & 358 & 17.77 & 19 & 19.28 & 155 & 11.55 \\ 7 & 1045 & 32.10 & 19 & 19.65 & 155 & 7.98\end{array}$

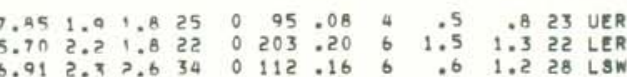

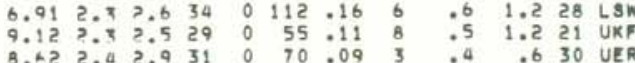

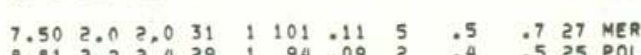

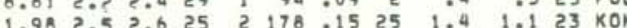

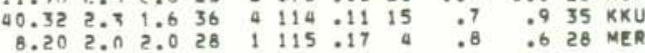
$\begin{array}{llllllllllll}9 . A B & 3.0 & 3.3 & 34 & 1 & 72 & .09 & 4 & .4 & .4 & 32 & \\ 0.9 E R\end{array}$

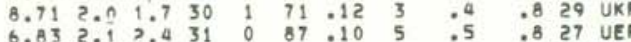

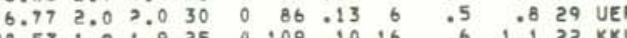

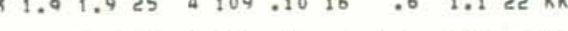
$\begin{array}{llllllllllll}5.03 & 2.0 & 2.1 & 23 & 3 & 143 & .32 & 5 & 1.1 & 2.2 & 18 & \mathrm{kON}\end{array}$

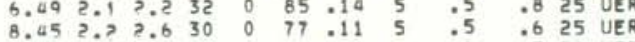

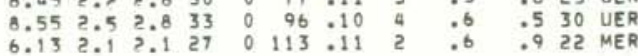
$\begin{array}{llllllllllll}5.10 & 2.4 & 2.4 & 29 & 0 & 87 & .14 & 5 & .6 & 2.1 & 26 & 6 \text { UER }\end{array}$

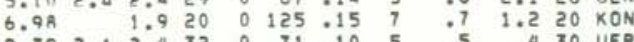

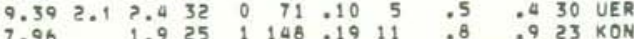

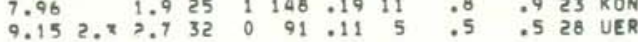
$\begin{array}{llllllllllll}9.38 & 2.0 & 3.2 & 37 & 2 & 96 & .12 & 5 & .5 & .4 & 27 & \text { UER }\end{array}$

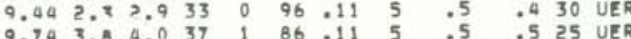

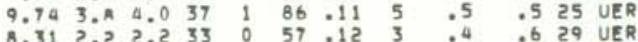

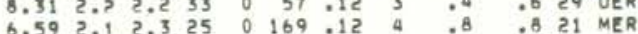

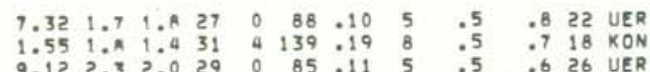

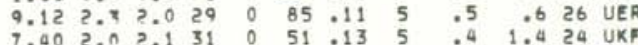

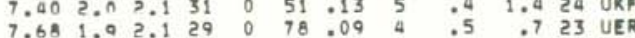

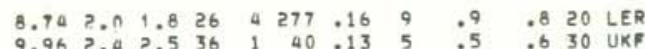

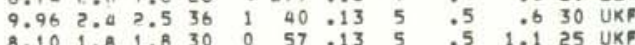

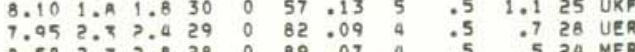

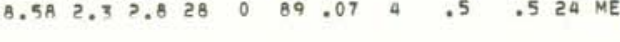

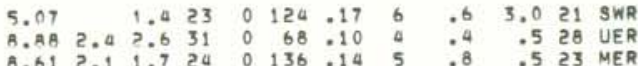

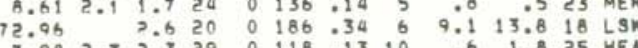

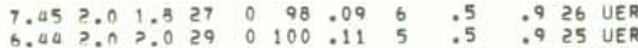

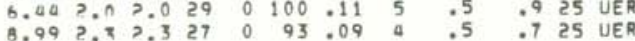

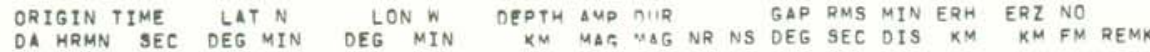

1978 DEC ? $1838 \quad 54.72 \quad 1921.41 \quad 155 \quad 2.72$ उ21 $18.971920 .73 \quad 155$ 6.06

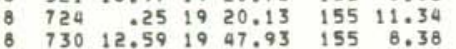
$\begin{array}{lllllll}8 & 910 & 3.01 & 19 & 19.22 & 155 & 11.94\end{array}$ $810 \quad 25.21$ 19 23.85 15524.80

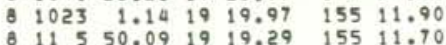

$15358.9919 .23 .41 \quad 155 \quad 4.02$

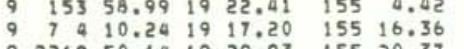
$9221950.141929 .93 \quad 15520.37$

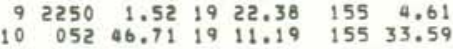

$\begin{array}{lllllll}10 & 1228 & 48.78 & 19 & 19.39 & 155 & 9.83\end{array}$ $\begin{array}{lllllll}10 & 1228 & 48.78 & 19 & 19.39 & 155 & 9.83 \\ 10 & 1325 & 2.30 & 19 & 20.38 & 155 & 6.73\end{array}$ $10 \quad 162942.3919 \quad 10.87 \quad 15541.70$ $\begin{array}{rrrrrrr}10 & 1835 & 25.13 & 19 & 20.16 & 155 & 6.92 \\ 11 & 713 & 19.85 & 19 & 22.02 & 155 & 4.28\end{array}$

$\begin{array}{lllllll}11 & 821 & 13.06 & 19 & 22.97 & 155 & 4.57\end{array}$ $11 \quad 92852.77 \quad 1922.71$ 155 4.46 $\begin{array}{lllllll}11 & 1159 & 0.01 & 19 & 26.57 & 155 & 30.24 \\ 11 & 2315 & 38.17 & 19 & 17.50 & 155 & 30.80\end{array}$ 120411.60 19 22.05 $155 \quad 7.15$ $\begin{array}{lllllll}12 & 116 & 55.14 & 19 & 6.33 & 155 & 27.27 \\ 12 & 253 & 23.98 & 19 & 29.71 & 150 & 57.18\end{array}$ $12184431.65 \quad 1921.75 \quad 155 \quad 4.60$ 12 $213939.121923 .23 \quad 1553.40$

$\begin{array}{llllllll}13 & 8 & 5 & 42.92 & 19 & 25.31 & 155 & 25.02\end{array}$

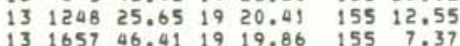
$13165746.411919 .86 \quad 155 \quad 7.37$ $1319737.471916 .86 \quad 15512.47$

$\begin{array}{lllllll}14 & 416 & 35.26 & 19 & 19.06 & 155 & 14.01\end{array}$

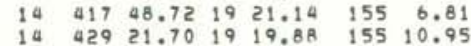
$14162015.501918 .53 \quad 155 \quad 13.34$ 14 $172016.051919 .16 \quad 15513.02$

$\begin{array}{lllllll}14 & 2034 & 47.27 & 19 & 20.74 & 155 & 10.92 \\ 15 & 129 & 22.42 & 19 & 30.36 & 155 & 17.03\end{array}$

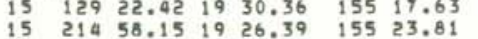
$1532030.281921 .27 \quad 155 \quad 5.86$ $\begin{array}{lllll}5 & 62 & 10.12 \quad 19 & 34,33 \quad 155 & 41.67\end{array}$ $\begin{array}{lllllll}15 & 737 & 50.42 & 19 & 19.99 & 155 & 13.14 \\ 15 & 915 & 48.07 & 19 & 22.92 & 155 & 3.74\end{array}$ $15 \quad 1050 \quad 40.5919 \quad 19.81 \quad 155 \quad 8.27$

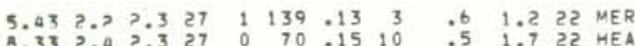

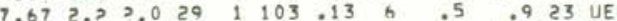

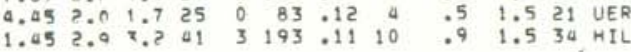

$\begin{array}{llllllllllll}9.79 & 3.2 & 3.4 & 35 & 0 & 98 & .10 & 5 & .5 & .4 & 32 & 32 \\ \text { UER }\end{array}$

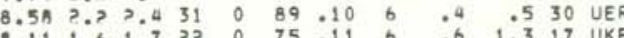
\begin{tabular}{llllllllll}
1.6 & 1.7 & 22 & 0 & 75 & 11 & 6 & .6 & 1.3 & 17 \\
\hline
\end{tabular}

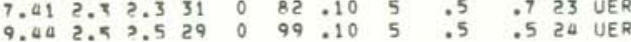
$\begin{array}{llllllllllll}0.22 & 2.0 & 1.9 & 27 & 0 & 87 & .08 & 4 & .6 & .5 & 22 & \text { MER }\end{array}$

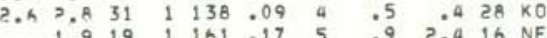

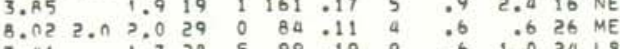

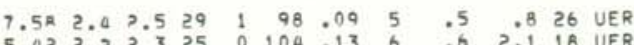

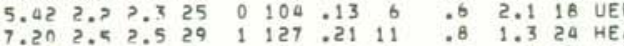
$\begin{array}{llllllllllll}7.60 & 2.1 & 1.8 & 27 & 0 & 107 & 0.10 & 6 & .5 & .9 & 24 & \text { UER } \\ 9.40 & 2.0 & 5.2 & 33 & 1 & 89 & .08 & 5 & .6 & .4 & 30 & \text { MER }\end{array}$

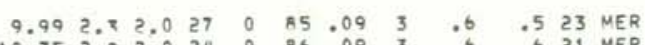

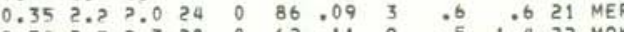

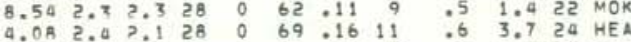
$5.502 .07 .521 \quad 1 \quad 72.1450 .61 .3$ 20 UER

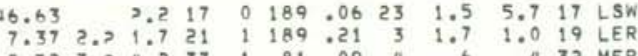
\begin{tabular}{rrrrrrrrrrr}
7.72 & 3.9 & 0.2 & 37 & 1 & 81 & .09 & 4 & .6 & .4 & 32 \\
7.29 & 1.0 & 2.0 & 23 & 0 & 104 & .12 & 3 & .6 & .9 & 20 \\
\hline
\end{tabular} $7,332.52 .032 \quad 148.13 \%$

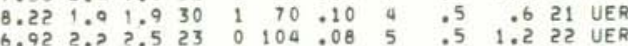

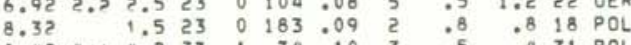
$\begin{array}{llllllllll}3.97 & 99 & 11 & 0 & 106 & -10 & 4 & .6 & 1.9 & 9 \\ 0 & \text { UER }\end{array}$

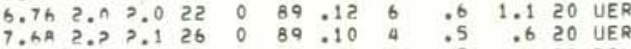

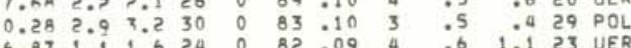

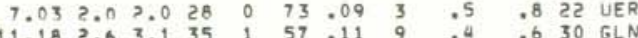

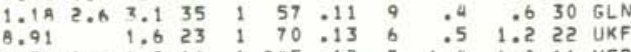

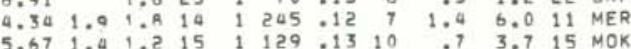

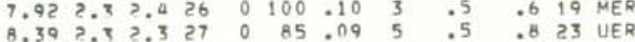

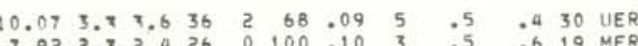




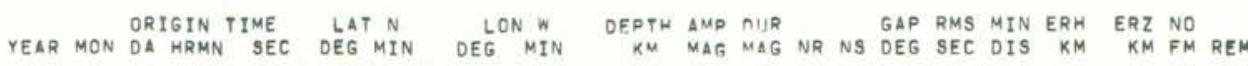

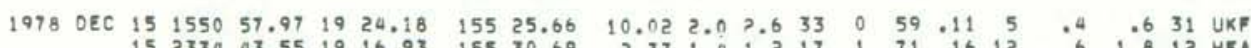

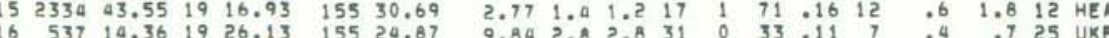

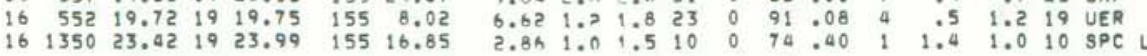

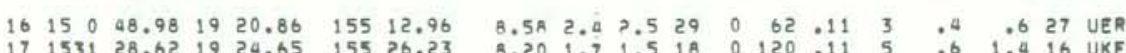

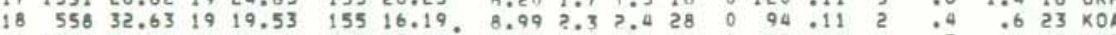

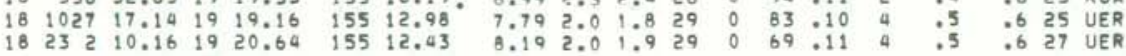

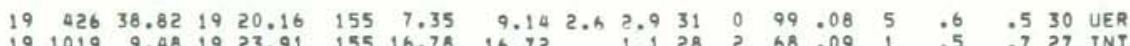

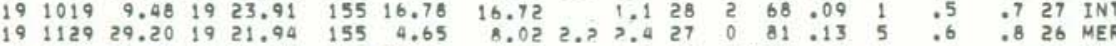

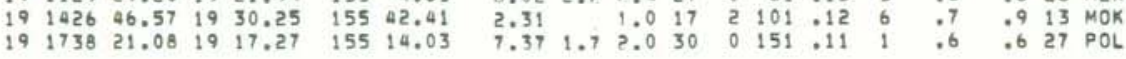

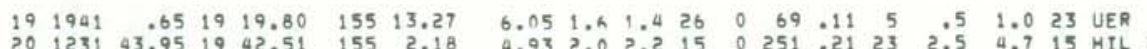

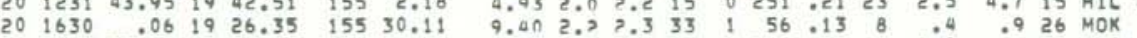

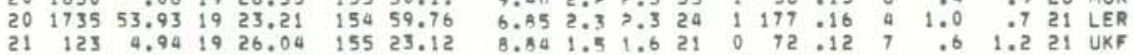

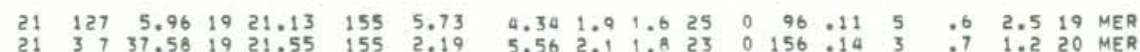

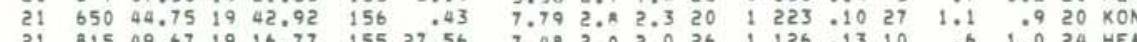

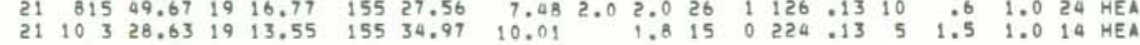

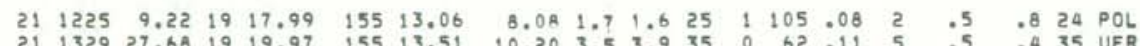

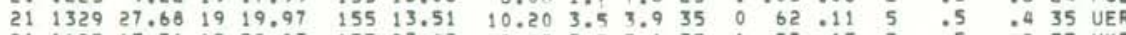

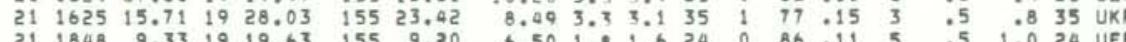

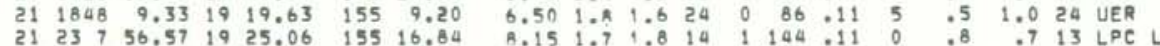

$22 \quad 149 \quad 48.07 \quad 1912.02 \quad 15533.21$ $22 \quad 436 \quad 09.97 \quad 1920.43 \quad 15512.51$

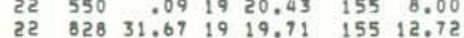
22 82831.67 19 19.71 155 12.72

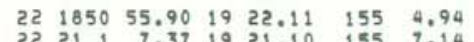

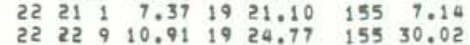
$2324016.051912 .09 \quad 15532.85$ $37.43 \quad 19 \quad 17.26 \quad 15521.58$ $\begin{array}{rrrrrrr}23 & 443 & 40.27 & 19 & 11.79 & 155 & 32.42 \\ 23 & 1026 & 38.99 & 19 & 26.36 & 155 & 49.42\end{array}$ $231323 \quad 4.521921 .96 \quad 155 \quad 4.81$ $231037 \quad 12.08 \quad 1920.95 \quad 155$ 19.45

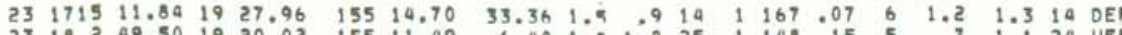

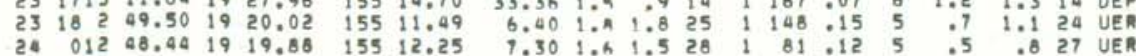

YEAR MON ORIGIN TIME LRMN SEC DEG MIN DEG MIN DEPTH AMP RIR NR GAP RMS MIN ERH ERZ NO 1978 DEC $24 \quad 029 \quad 97.17 \quad 1922.15 \quad 15526.38$ $24 \quad 130 \quad 34.67 \quad 1922.37 \quad 15529.70$

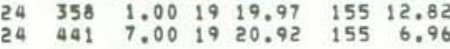
$\begin{array}{lllllll}24 & 710 \quad .85 \quad 19 & 19.49 \quad 155 \quad 7.48\end{array}$

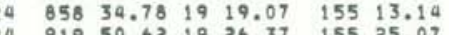
$91950.621926 .37 \quad 15525.07$

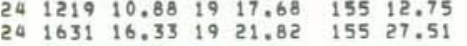
$24 \quad 1716 \quad 58.07 \quad 19 \quad 17.02 \quad 155 \quad 23.66$

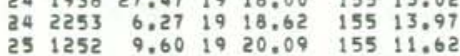

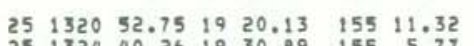

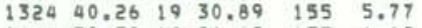
$\begin{array}{lllllll}25 & 1628 & 50.32 & 19 & 21.19 & 155 & 4.62 \\ 25 & 1712 & 45.00 & 19 & 19.92 & 155 & 7.09\end{array}$ $252138 \quad 54.441923 .52 \quad 15524,13$

25 $2258 \quad 47.34 \quad 1920.61 \quad 155 \quad 2.40$ $26042 \quad 1.1420 \quad 2.86 \quad 15534.79$

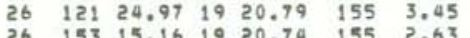

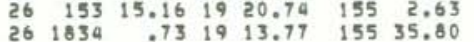

$27 \quad 040 \quad 55.83 \quad 1920.13 \quad 15512.94$ $\begin{array}{lllllll}27 & 049 & 8.20 & 19 & 20.05 & 155 & 11.86\end{array}$

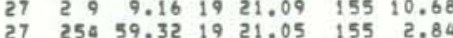
$\begin{array}{lllllll}27 & 254 & 59.32 & 19 & 21.05 & 155 & 2.84 \\ 27 & 330 & 36.59 & 19 & 13.92 & 155 & 37.30\end{array}$

$\begin{array}{lllllll}27 & 69 & 39.91 & 19 & 26.30 & 154 & 53.37 \\ 27 & 1452 & 16.90 & 19 & 19.29 & 155 & 12.05\end{array}$ $\begin{array}{lllllll}27 & 1452 & 16.90 & 19 & 19.29 & 155 & 12.05 \\ 27 & 1510 & 54.57 & 19 & 17.85 & 155 & 15.4\end{array}$ $27161929.491921 .54 \quad 155 \quad 13.05$

$\begin{array}{lllllll}28 & 036 & 48.15 & 19 & 19.89 \quad 155 & 10.77\end{array}$

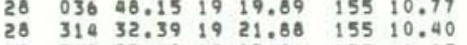
$\begin{array}{lllllll}28 & 315 & 27.40 & 19 & 15.06 & 155 & 6.15 \\ 28 & 615 & 19.70 & 19 & 20.97 & 155 & 2.94\end{array}$ $28 \quad 740 \quad 21.4019 \quad 19,95 \quad 15511,70$ $\begin{array}{lllllll}28 & 756 & 19.40 & 19 & 19.90 & 155 & 11.66 \\ 28 & 851 & 1.88 & 19 & 21.65 & 155 & 15.21\end{array}$

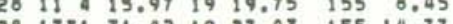
$28173131.42 \quad 1927.03 \quad 15514.77$

$\begin{array}{lllllll}29 & 017 & 56.40 & 19 & 19.86 & 155 & 10.36\end{array}$ $\begin{array}{lllllll}29 & 738 & 49.44 & 19 & 19.88 & 155 & 8.05 \\ 29 & 739 & 36.95 & 19 & 20.01 & 155 & 8.27\end{array}$

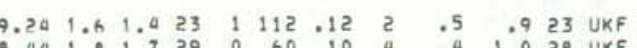

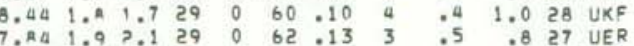
$\begin{array}{llllllllllll}7.65 & 1.5 & 1.6 & 26 & 1 & 73 & .13 & 5 & .6 & .7 & 25 & \text { UER } \\ 7.45 & 1.4 & 7.4 & 26 & 0 & 91 & .13 & 6 & .6 & .9 & 26 & \text { UER }\end{array}$ $\begin{array}{lllllllllllll}3.21 & 1.9 & 1.8 & 29 & 0 & 108 & -17 & 4 & .6 & 1.6 & 27 & \text { UER }\end{array}$

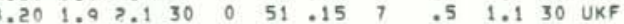
$\begin{array}{rllllllllllll}7.46 & 1.7 & 1.4 & 22 & 0 & 133 & .08 & 2 & .6 & 1.1 & 22 & \text { POL } \\ 47.93 & 1.9 & 1.6 & 26 & 2 & 94 & .11 & 1 & .9 & 1.3 & 26 & \text { HEA }\end{array}$

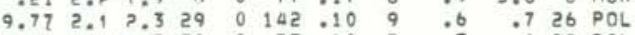
$\begin{array}{llllllllllll}6.61 & 1.4 & 1.7 & 30 & 0 & 73 & .10 & 3 & .5 & .8 & 29 & \text { POL } \\ 7.99 & 1.9 & 2.1 & 30 & 0 & 82 & .11 & 5 & .5 & .7 & 26 & \text { UER }\end{array}$

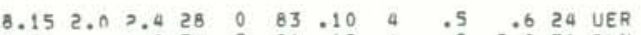

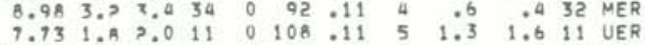
$10.522 .52 .816 \quad 0 \quad 90.12 \quad 6 \quad 96 \quad .8$ 15 UKF

$\begin{array}{lllllllllllllllll}7.61 & 2.1 & 2.3 & 16 & 0 & 173 & .11 & 2 & 1.1 & .5 & 12 & \text { MER }\end{array}$

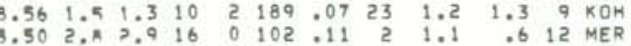
7.462 .01 .712 O 155.11 2 1.25 .8 11 MER $9.93 \quad 4.79 .635 \quad 0 \quad 70.08 \quad 5 \quad .5 \quad .434$ UER

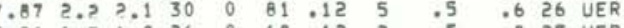

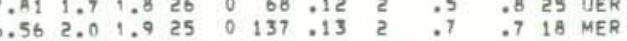

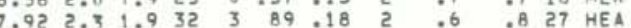

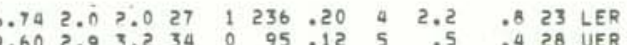

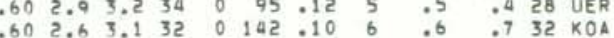

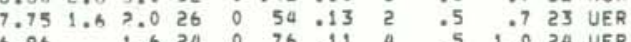

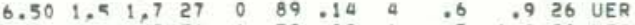

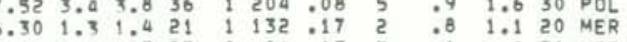
$\begin{array}{llllllllllll}.44 & 2.1 & 2.5 & 33 & 0 & 86 & 0.15 & 5 & .5 & .7 & 27 & \text { UER } \\ .41 & 3.4 & 3.4 & 34 & 2 & 62 & .16 & 2 & .4 & .5 & 28 & \text { KOA }\end{array}$

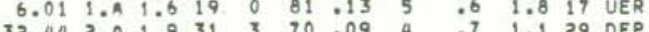

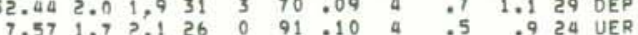

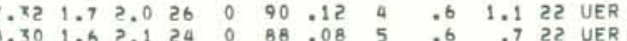

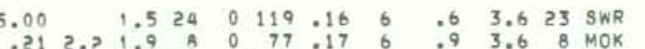

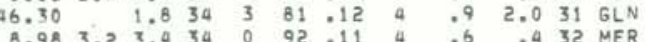

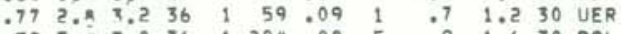


ORIGIN TIME LAT N LON W YEAR MON DA HRMN SEC DEG MIN DEG MIN $\begin{array}{lllllllll}1978 & \text { DEC } 29 & 9 & 9 & 55.80 & 19 & 26.97 & 155 & 14.71\end{array}$ $29 \quad 1043 \quad 49.63 \quad 1922.85 \quad 155 \quad 3.19$ $29 \quad 1333 \quad 55.37 \quad 19 \quad 28.37 \quad 154 \quad 53.05$ $\begin{array}{llllllll}29 & 1451 & 17.97 & 19 & 49.03 & 156 & 17.25\end{array}$ $29 \quad 1456 \quad 44.06 \quad 1950.07 \quad 156 \quad 21.44$ $29 \quad \begin{array}{lllll}1523 & 44.19 & 19 & 20.95 \quad 155 \quad 10.85\end{array}$ $\begin{array}{lllllll}29 & 1652 & 9.35 & 19 & 7.35 & 155 & 24.21\end{array}$ $\begin{array}{lllllll}29 & 2017 & 1.05 & 19 & 19.76 & 155 & 7.64\end{array}$ $292231 \quad 44.38 \quad 1921.34 \quad 155 \quad 6.66$

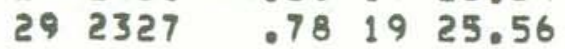
$29 \quad 2356 \quad 14.351929 .07$ $\begin{array}{llllll}30 & 0 & 4 & 54.89 & 19 & 21.59\end{array}$

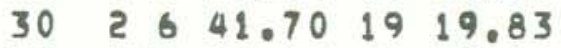
$30 \quad 43956.69 \quad 19 \quad 28.10$

$\begin{array}{llll}30 & 553 & 52.22 \quad 19 & 20.66\end{array}$

$\begin{array}{lllll}30 & 648 & 14.51 & 19 & 25.67\end{array}$

$\begin{array}{llllll}30 & 744 & 17.46 & 19 & 19.49\end{array}$

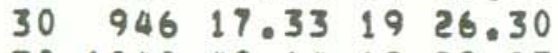

$\begin{array}{llllll}30 & 1840 & 48.14 & 19 & 22.85\end{array}$

$\begin{array}{lllll}31 & 248 & 39.41 & 19 & 31.92\end{array}$

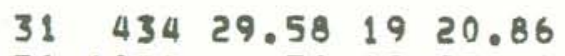
$\begin{array}{lllll}31 & 1039 & .30 & 19 & 27.44\end{array}$

$\begin{array}{llllll}31 & 1939 & 43.04 & 19 & 20.21\end{array}$

$\begin{array}{lllll}31 & 1945 & 12.55 & 19 & 30.03\end{array}$

$\begin{array}{lllll}312232 & 7.61 & 1920.47\end{array}$

15524.46

15451.07

1551.94

15511.53

15453.42

15510.86

15526.55

15511.71

15451.04

1554.12

15541.90

15513.06

15454.33

15511.58

15529.06

15513.02
DEPTH AMP OIIR GAP RMS MIN ERH KM MAG MAG NR NS DEG SEC DIS KM

ERZ NO

$\begin{array}{rrrrrrrrrrrr}31.55 & 1.5 & 2.1 & 26 & 1 & 127 & .07 & 4 & .8 & 1.4 & 21 & \text { DEP } \\ 7.73 & 1.8 & 1.9 & 19 & 1 & 114 & .13 & 4 & .6 & 1.4 & 14 & \text { MER } \\ 5.01 & 1.3 & 1.4 & 17 & 2 & 258 & .14 & 1 & 1.9 & .6 & 9 & \text { LER } \\ 6.36 & 3.4 & 2.9 & 28 & 0 & 322 & .11 & 49 & 15.7 & 5.5 & 26 & \text { DIS } \\ 2.29 & 2.7 & 3.1 & 29 & 0 & 322 & .11 & 57 & 11.2 & 11.4 & 22 & \text { DIS }\end{array}$

$8.341 . ? 1.418$

071.08 $44.18 \quad 1.5 \quad 1.7 \quad 19$ $6.96 \quad 1.0 \quad 1.319$ 6.601 .41 .820 0184.05 1187.086 9.692 .32 .730

187.10
$5.491 .5 \quad 1.3 \quad 15$

7.342 .92 .625

8.631 .11 .421

4.931 .21 .117

8.491 .51 .824

8.902 .72 .935

$7.301 .1 \quad .718$

8.141 .71 .723

$10.23 \quad 1.6 \quad 1.6 \quad 22$

9.483 .02 .932

6.661 .82 .227

4.52 ?. 11.920

$6.652 .42 .6 \quad 34$

$2.68 \quad 1.6 \quad 1.6 \quad 21$

8.421 .61 .828

$$
\begin{array}{rrrl}
.5 & 1.1 & 11 & \text { UER } \\
1.4 & 3.2 & 16 & \text { LSW L } \\
.9 & 1.0 & 16 & \text { UER } \\
.5 & 1.2 & 16 & \text { UER } \\
.4 & .8 & 27 & \text { UKF } \\
2.5 & .9 & 6 & \text { LER } \\
.7 & .6 & 17 & \text { MER } \\
.5 & 1.0 & 17 & \text { UER } \\
1.5 & .7 & 8 & \text { LER } \\
.4 & .7 & 19 & \text { UER }
\end{array}
$$

.5 .832 UKF .51 .216 UER $2.7 \quad .716$ LER

.5 .821 MER

.5 .728 MOK

. 81.116 UER 2.1 1.2 19 LER $.6 \quad .927$ UER $.51 .6 \quad 14$ NER .4 .624 UER 
TABLE 6. HVO EARTHQUAKE SUMMARY LIST $(M>3)$

PAGE 1

ORIGIN TIME LAT N LON W YEAR MON DA HRMN SEC DEG MIN DEG MIN

1978 JAN $2220049.73 \quad 1920.03$

$4842 \quad 27.331919 .43$

$\begin{array}{llllll}4 & 10 & 0 & 23.66 & 19 & 20.45\end{array}$

$\begin{array}{llllll}5 & 358 & 46.70 & 19 & 18.96\end{array}$

5121356.021919 .03

$72030 \quad 35.92 \quad 1959.60$ $\begin{array}{llll}10 & 223311.4519 & 19.55\end{array}$

$\begin{array}{lllll}10 & 239 & 54.40 & 19 & 19.63\end{array}$

$\begin{array}{llllll}12 & 1434 & 6.57 & 19 & 31.18\end{array}$

13104010.721918 .98

1401518.891911 .00

$\begin{array}{lllll}16 & 1643 & 46.56 & 19 & 20.88\end{array}$

19155210.491945 .62

$\begin{array}{llllllll}21 & 12 & 2 & 26.21 & 19 & 21.37\end{array}$

22 54132.821918 .84

$\begin{array}{llllll}22 & 2021 & 38.70 & 19 & 19.91\end{array}$

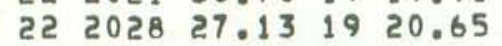

$\begin{array}{lllll}23 & 056 & 10.31 & 19 & 20.17\end{array}$

$\begin{array}{lllll}24 & 1518 & 17.04 & 19 & 20.47\end{array}$

$2531943.44 \quad 1918.79$

$\begin{array}{lllll}26 & 1938 & 21.21 & 19 & 26.65\end{array}$

$\begin{array}{lllll}28 & 1632 & 59.51 & 19 & 19.82\end{array}$

$\begin{array}{llllll}29 & 818 & 56.79 & 19 & 21.51\end{array}$

$\begin{array}{llllll}30 & 038 & 2.57 & 19 & 22.00\end{array}$

$\begin{array}{llllllll}\text { FEB } & 2 & 11 & 3 & 47.79 & 19 & 20.07\end{array}$

$$
\begin{array}{rrrrr}
2 & 1621 & 21.66 & 19 & 19.68 \\
2 & 1932 & 45.99 & 19 & 26.48 \\
5 & 138 & 40.41 & 19 & 19.93 \\
6 & 1241 & 46.94 & 19 & 20.25 \\
7 & 939 & 14.23 & 19 & 19.42
\end{array}
$$

$\begin{array}{lllll}7 & 1532 & 26.38 & 19 & 40.34\end{array}$

$\begin{array}{llllll}8 & 1156 & 37.30 & 19 & 20.75\end{array}$

125614.471911 .91

141056.391924 .00

$15 \quad 63734.751920 .18$

$18 \quad 145159.76 \quad 19 \quad 19.64$ 199151.791920 .74

$\begin{array}{lllll}19 & 1149 & 47.68 & 19 & .63\end{array}$

$\begin{array}{lllll}20 & 734 & 24.25 & 19 & 19.34\end{array}$

$\begin{array}{lllll}21 & 156 & 55.90 & 19 & 21.93\end{array}$

$22 \quad 74355.091926 .06$

$\begin{array}{llllll}23 & 6 & 6 & 11.12 & 19 & 19.49\end{array}$

$\begin{array}{llllll}24 & 129 & 39.97 & 19 & 19.17\end{array}$

$\begin{array}{lllll}24 & 837 & 4.65 & 19 & 26.51\end{array}$

$\begin{array}{lllll}24 & 2255 & 28.17 & 19 & 27.25\end{array}$

$\begin{array}{rrrrrr} & 25 & 929 & 25.39 & 19 & 20.07 \\ \text { MAR } & 2 & 051 & 3.04 & 19 & 19.88 \\ & 3 & 2019 & 4.24 & 20 & 23.00\end{array}$
$155 \quad 13.25$

$155 \quad 10.83$

$155 \quad 13.70$

$155 \quad 15.97$

15459.91

15520.50

15513.06

15513.37

$155 \quad 55.53$

15512.25

15519.84

$155 \quad 6.65$

1556.43

$155 \quad 2.91$

1557.13

1553.54

$155 \quad 3.87$

$155 \quad 3.49$

$155 \quad 6.41$

$155 \quad 23.57$

15548.08

15510.24

155.46

$156 \quad 22.19$

15514.01

1557.89

$155 \quad 18.14$

$155 \quad .46$

1557.99

1559.92

$155 \quad 3.42$

1556.12

$155 \quad 36.55$

15516.85

15556.64

1558.62

15513.49

15525.72

1557.68

1555.69

$155 \quad 34.41$

1556.22

15510.40

15559.94

$156 \quad 15.25$

$155 \quad 13.61$

15513.35

15619.22

DEPTH AMP NIIR KM MAG MAG NR NS DEG SEC DIS KM

$9.862 .4 \quad 3.230$

$9.93 \quad 3.7 \quad 11.135$

$9.202 .7 \quad 3.434$

$9.973 . ? \quad 2.438$

$44.072 .8 \% .034$

9.712 .93 .233

$10.16 \quad 3.8 \quad 4.138$

9.472 .23 .233

$9.953 .0 \quad 3.132$

8.8 B 2.63 .032

$\begin{array}{lllll}51.68 & 2.4 & 3.137\end{array}$

7.93
$2.7 \quad 3.232$

$39.00 \quad 3.0 \quad 3.2 \quad 37$

$\begin{array}{lllll}7.27 & 2.7 & 3.1 & 34\end{array}$

8.693 .53 .637

9.423 .74 .136

9.333 .64 .038

$8.842 .3 \quad 3.0 \quad 32$

8.793 .23 .439

5.962 .43 .135

6.32 2.73.130

$7.16 \quad 2.2 \quad 3.331$

$7.40 \quad 2.5 \quad 3.130$

$6.26 \quad 3.2 \quad 2.941$

$33.45 \quad 3.4 \quad 3.3 \quad 37$

$9.27 \quad 3.0 \quad 3.132$

$15.453 .5 \times .537$

$6.92 \quad 2.83 .233$

$8.98 \quad 2.7 \quad 3.131$

0.332 .43 .033

$\begin{array}{lllll}1.77 & 2.43 .126\end{array}$

8.912 .72 .029

$\begin{array}{lllll}8.96 & 3.9 & 2.8 & 34\end{array}$

$15.023 .4 \times .740$

$14.952 .9 \quad 3.5 \quad 27$

$9.30 \quad 3.0 \quad 3.6 \quad 32$

$9.972 .7 \quad 3.235$

41.203 .12 .137

$8.862 .6 \times 134$

8.153 .33 .437

$47.55 \quad 2.5 \quad 2.0 \quad 17$

9.313 .13 .236

$9.61 \quad 2.93 .138$

25.52 $3.1 ? .831$

16.893 .23 .133

$9.842 .93 .3 \quad 36$

$9.72 \quad 3.74 .030$

$14.262 .9=2.234$ $\begin{array}{rrrr}1 & 66 & .08 \\ 1 & 100 & .10 \\ 2 & 57 & .12 \\ 2 & 100 & .10 \\ 2 & 220 & .10\end{array}$

$\begin{array}{llll}2 & 274 & .13 \quad 27\end{array}$

2 $75.10 \quad 5$

169.10

$0 \quad 218.22$

1100.11

$5 \quad 178 \quad .10 \quad 13$

095.126

2 $187.10 \quad 5$

2 132.113

2134.123

$1150.11 \quad 1$

3101.11

2122.11

3107.12

1104.15

191.20

$0 \quad 90.10$

$0 \quad 195.11$

5244.1349

160.105

$395.10 \quad 4$

158.112

3215.13

0.97 .09

1196.1719

1103.106

192.256

$3 \quad 35.112$

$2 \quad 234 \quad .20 \quad 11$

$\begin{array}{llll}0 \quad 77.10 \quad 4 & 40\end{array}$

$157.10 \quad 4$

$1215.06 \quad 17$

2 $106.10 \quad 4$

379.125

$274.14 \quad 4$

2. 166.115

2 106.135

$\begin{array}{llll}2 & 231.09 & 10\end{array}$

$0263.16 \quad 35$

54
GAP RMS MIN ERH

86.10

ERZ NO

KM FM REMK

$$
\begin{array}{rrrr}
.4 & .4 & 29 & \text { UER } \\
.4 & .5 & 28 & \text { UER F } \\
.4 & .6 & 29 & \text { UER } \\
.4 & .4 & 33 & \text { KOA F } \\
1.1 & 1.9 & 29 & \text { DIS }
\end{array}
$$

$$
\begin{array}{rrrrr}
1.9 & .8 & 28 & \text { KKU F } \\
.4 & .430 & \text { UER F } \\
.4 & .428 & 28 \text { UER } \\
1.5 & .728 & \text { KON F } \\
.4 & .628 \text { POL }
\end{array}
$$

$\begin{array}{rrrl}.8 & 1.0 & 26 & \text { HLP L } \\ .5 & .7 & 29 & \text { UER } \\ .8 & 1.0 & 27 & \text { HIL } \\ .5 & .6 & 31 & \text { MER } \\ .5 & .4 & 33 & \text { POL }\end{array}$

.7 .434 MER F

.5 .433 MER F

$.6 \quad .527$ MER

.4

.635 UER F

1.031 SWR

$\begin{array}{llll}.6 & 1.327 \mathrm{KON}\end{array}$

.5 .728 UER

.9 .628 LER

.91 .336 DIS

.7 .930 DEP

$.4 \quad .727$ UER

$.4 \quad .330$ INT F

.5 .626 DIS

.5 .630 UER

$.5 \quad .629$ UER

$1.11 .525 \mathrm{HIL} B$

.5 .620 UER

.81 .123 HEA

.4 .233 INT F

$2.41 .0 .26 \mathrm{KON}$

$$
\begin{array}{rrrr}
.5 & .4 & 31 & \text { UER } \\
.4 & .5 & 30 & \text { UER } \\
1.0 & 2.0 & 35 & \text { LSW } \\
.4 & .6 & 31 & \text { UER } \\
.4 & .7 & 30 & \text { MER } \\
2.0 & 1.5 & 14 & \text { MOK } \\
.5 & .4 & 32 & \text { UER } \\
.4 & .5 & 32 & \text { UER } \\
1.1 & 1.6 & 18 & \text { KON } \\
3.5 & 98.1 & 29 & \text { DIS F }
\end{array}
$$

$$
\begin{array}{rrrr}
3 & 61 & .10 & 5 \\
3 & 67 & .10 & 5 \\
6 & 210 & .14 & 43
\end{array}
$$


ORIGIN TIME LAT N YEAR MON DA HRMN SEC DEG MIN 1978 MAR

$\begin{array}{lllll}5 & 1926 & 16.56 & 19 & 26.03\end{array}$

$\begin{array}{llllllll}6 & 15 & 3 & 34.53 & 19 & 22.78\end{array}$

$\begin{array}{llllllll}6 & 15 & 6 & 43.39 & 19 & 22.95\end{array}$

$\begin{array}{lllll}7 & 1546 & 20.75 \quad 19 & 27.09\end{array}$

$10 \quad 524 \quad 43.38 \quad 1919.59$

$\begin{array}{lllll}10 & 2155 & 51.22 \quad 19 & 26.14\end{array}$

$\begin{array}{lllllll}12 & 23 & 8 & 18.57 & 19 & 27.79\end{array}$

$\begin{array}{llllll}13 & 1947 & 46.87 & 19 & 23.15\end{array}$

$\begin{array}{lllll}14 & 723 & 13.27 & 19 & 22.09\end{array}$

1410928.381919 .83

$\begin{array}{lllll}17 & 229 & 1.71 & 19 & 26.77\end{array}$

$17246 \quad 14.09 \quad 1920.14$

$20 \quad 1913 \quad 25.591921 .99$

$\begin{array}{llllll}24 & 13630.09 & 19 & 27.74\end{array}$

$\begin{array}{llll}25 & 65756.431920 .88\end{array}$

$26 \quad 1912 \quad 57.29 \quad 19 \quad 10.34$

$27 \quad 41952.71 \quad 1920.88$

$27 \quad 1131 \quad 8.66 \quad 1921.12$

$\begin{array}{llllll}27 & 2157 & 50.96 & 19 & 28.02\end{array}$

$28 \quad 1542 \quad 46.11 \quad 18 \quad 49.13$

$\begin{array}{llllll}29 & 156 & 35.78 & 19 & 21.46\end{array}$

$\begin{array}{llllll}29 & 2 & 1 & 23.27 & 19 & 17.67\end{array}$

$29 \quad 174923.14 \quad 1919.73$

$\begin{array}{lllll}31 & 523 & 41.50 & 19 & 24.92\end{array}$

$\begin{array}{lllllll}A P R & 2 & 1316 & 49.70 & 19 & 18.99\end{array}$

$\begin{array}{lllll}2 & 1828 & 32.98 & 19 & 19.90\end{array}$

$\begin{array}{lllll}3 & 237 & 46.80 & 19 & 18.75\end{array}$

$\begin{array}{llllll}4 & 8 & 0 & 22.26 & 19 & 9.44\end{array}$

$\begin{array}{llllll}6 & 347 & 14.06 \quad 19 & 19.68\end{array}$

7164343.291952 .38

$9 \quad 129 \quad 27.02 \quad 1922.67$ $\begin{array}{llllll}10 & 244 & 39.71 & 19 & 19.10\end{array}$ $10 \quad 212357.831929 .16$ $1293528.10 \quad 1921.26$ $12 \quad 205512.67 \quad 1920.83$

$14 \quad 030 \quad 50.88 \quad 19 \quad 18.13$

$\begin{array}{lllllll}17 & 0 & 3 & 17.40 & 19 & 25.24\end{array}$

$17 \quad 247 \quad 49.831921 .22$

$\begin{array}{lllll}17 & 321 & 12.23 & 19 & 20.17\end{array}$

$17 \quad 1840 \quad 54.66 \quad 1921.14$

$\begin{array}{lllll}18 & 448 & 55.04 \quad 19 & 20.63\end{array}$

$19 \quad 1636 \quad 25.05 \quad 19 \quad 12.18$

$\begin{array}{llllll}20 & 2349 & 3.53 & 19 & 19.53\end{array}$

$23 \quad 726 \quad 24.49 \quad 1921.14$

$24 \quad 126 \quad 48.36 \quad 1922.64$

$\begin{array}{llllll}24 & 232 & 1.06 \quad 19 & 21.90\end{array}$

$\begin{array}{lllll}28 & 134 & 37.55 & 20 & 9.51\end{array}$

MAY $3729 \quad 6.40 \quad 1917.49$
LON W DEG MIN

15535.36

$155 \quad 5.48$

1555.44

15526.93

1557.99

15536.43

15529.29

15516.12

15515.50

1556.74

15524.68

15513.08

15525.21

15452.68

15510.74

15528.97

1558.00

15525.80

15450.60

15512.05

$155 \quad 6.56$

$155 \quad 12.93$

1559.28

15528.81

15515.66

15512.01

15515.64

15536.04

1558.05

1557.68

$155 \quad 4.70$

15512.30

15552.84

1557.14

$\begin{array}{ll}155 & 3.77\end{array}$

15545.51

15516.80

15514.85

15513.81

$155 \quad 1.44$

1553.86

15531.25

15511.67

15515.37

$155 \quad 4.87$

$155 \quad 4.04$

15535.84

15529.96

DEPTH AMP DIIR KM MAG MAG NR NS OEG SEC. DIS KM

ERZ NO

$36.612 .4 \quad 3.4 \quad 24$ .142 .63 .531 $1.48 \quad 2.5 \quad 3.4 \quad 29$ 27.532 .53 .338 $9.553 .1 \quad 3.3 \quad 37$

$46.47 \quad 2.8 \quad 3.3 \quad 20$ $10.78 \quad 2.9 \quad 3.2 \quad 38$ $2.66 \quad 2.3 \quad 3.221$ $27.23 \quad 2.8 \quad 3.140$ 10.032 .93 .435

$8.553 .2 \quad 3.535$ $9.753 .0 \quad 3.237$ $11.40 \quad 3.3 \quad 3.640$ $45.113 .0 ? .838$ $8.672 .6 \quad 3.034$

$\begin{array}{llll}33.88 & 2.9 \quad 3.139\end{array}$ $9.10 \quad 2.4 \quad 3.1 \quad 35$ 10.813 .13 .234 8.013 .13 .226 $16.34 \quad 3.5 \quad 3.234$

$8.38 \quad 2.7 \quad 3.132$ $\begin{array}{lllll}10.89 & 2.7 & 3.0 & 31\end{array}$ $9.212 .6 \quad 3.030$ $10.853 .3 \quad 3.235$ $9.442 .53 .0 \quad 33$

$8.80 \quad 3.3 \quad 3.436$ $9.792 .5 \quad 3.1 \quad 35$ 5.252 .13 .228 $10.08 \quad 3.4 \quad 3.6 \quad 38$ $39.274 .0 \quad 4.3 \quad 35$

8.06 2.h 3.130 $3.602 .5 \quad 3.034$ $6.552 .9 \times 128$ 9.253 .33 .634 0.433 .73 .938

$52.96 \quad 3.0 \quad 3.7 \quad 9$ $\begin{array}{lllll}3.86 & 2.6 & 3.2 & 27\end{array}$ $10.013 .0 \quad 3.636$ $9.00 \quad 2.53 .134$ 7.393 .33 .936

$6.96 \quad 3.1 \quad 3.4 \quad 33$ $37.46 \quad 2.7 \quad 3.0 \quad 38$ $9.503 .0 \quad 3.230$ $9.00 \quad 2.83 .032$ 8.322 .73 .031

$\begin{array}{llll}7.11 & 2.6 & 3.129\end{array}$ $35.593 .1 ? .840$ $\begin{array}{lllll}7.88 & 3.3 & 3.5 & 36\end{array}$

$\begin{array}{ll}72 & .14 \\ 71 & .22 \\ 71 & .18 \\ 39 & .10 \\ 93 & .10\end{array}$

0

$2 \quad 54.12$

2 57.11

$3 \quad 57.11$

2 117.09

$$
\begin{array}{rrrr}
1.0 & 2.4 & 16 & \text { MOK L } \\
.6 & 1.7 & 28 & \text { MER * } \\
.5 & 1.3 & 27 & \text { MER } \\
.5 & .9 & 33 & \text { UKF } \\
.4 & .5 & 30 & \text { UER }
\end{array}
$$

1.43 .320 MOK L

$.4 \quad .636$ UKF

$.3 .518 \mathrm{SPC}$

$.5 \quad .826$ DEP

.6 .529 UER

.4 .925 UKF .4 .526 UER

.4 .335 HEA F

.81 .031 LER

.4 .628 UER

$.81 .336 \mathrm{LSW}$ $.4 \quad .427$ UER

$.4 .630 \mathrm{HEA}$

$1.4 \quad .623$ LER

5.837 .132 PPL

$.4 \quad .528$ UER

.7 .626 POL

.6 .523 UER

.5 .633 UKF

$.5 .530 \mathrm{KOA}$

.4 .432 UER F

$.4 .431 \mathrm{KOA}$

.61 .524 HEA

.5 .431 UER F

$2.13 .4 \quad 32$ KKU F

$.4 \quad .626$ MER

.4 .431 UER

.8

$.926 \mathrm{KON} F$

.529 UER F

. 434 MER F

$5.73 .28 \mathrm{KON} L$

$.4 \quad .625$ SPC

.4 .432 UER

$.5 \quad .627$ UER

.6 .433 MER F

.6

.725 MER

$.71 .231 \mathrm{LSW}$

.5 .629 UER

$.4 .528 \mathrm{KOA}$

.4 .527 MER

.4 .628 MER

$.71 .535 \mathrm{KOH}$

.5 .833 HEA F 
ORIGIN TIME LAT N YEAR MON DA HRMN SEC DEG MIN

1978 MAY $6 \quad 812 \quad 39.391924 .51$

$9 \quad 1846 \quad 41.16 \quad 1920.17$

$\begin{array}{llllll}10 & 240 & 34.08 & 19 & 21.54\end{array}$

$10 \quad 1423 \quad 57.941919 .46$

$\begin{array}{llll}12 & 516 & 10.32 & 1920.26\end{array}$

1310924.651958 .23

$\begin{array}{lllll}15 & 336 & 41.27 & 19 & 21.85\end{array}$

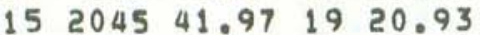

$19105948.91 \quad 1918.62$

$21 \quad 131751.60 \quad 1919.70$

$2221926.70 \quad 19 \quad 18.58$

$\begin{array}{llllll}22 & 2227 & 4.59 & 19 & 20.07\end{array}$

$\begin{array}{llllll}24 & 359 & 15.39 & 19 & 20.92\end{array}$

$\begin{array}{llllll}24 & 1652 & 52.98 & 19 & 21.06\end{array}$

$24 \quad 1939 \quad 47.83 \quad 1921.17$

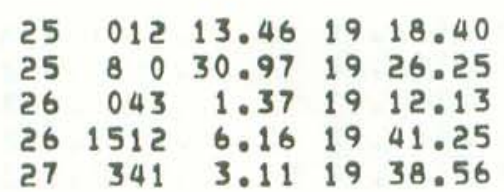

$\begin{array}{lllll}31 & 1437 & 5.62 & 19 & 21.11\end{array}$

$31 \quad 1916 \quad 55.47 \quad 19 \quad 19.57$

JUN $215 \quad 2 \quad 2.11 \quad 1940.01$

2 $1525 \quad 19.50 \quad 1941.89$

$\begin{array}{llllll}322 & 0 & 45.03 & 19 & 47.86\end{array}$

$4 \quad 121322.891922 .21$

$41235 \quad 59.20 \quad 1920.89$

$41248 \quad 16.221920 .23$

$\begin{array}{lllll}4 & 1840 & 43.33 & 19 & 19.75\end{array}$

$101852 \quad 22.011916 .72$

$1020 \quad 7 \quad 44.6319 \quad 19.87$

$\begin{array}{lllll}12 & 240 \quad .62 & 19 & 22.29\end{array}$

$\begin{array}{llllll}12 & 318 & 18.29 & 18 & 56.84\end{array}$

$\begin{array}{llllll}12 & 1157 & 12.12 & 19 & 24.02\end{array}$

$\begin{array}{llllll}12 & 1649 & 34.98 & 18 & 54.32\end{array}$

$\begin{array}{lllll}12 & 2238 & 37.04 & 19 & 25.09\end{array}$

1344240.841924 .83

$\begin{array}{llllll}13 & 654 & 1.15 & 19 & 20.47\end{array}$

$\begin{array}{llllll}13 & 1941 & 11.48 & 19 & 20.57\end{array}$

$\begin{array}{lllll}17 & 1734 & 23.67 \quad 19 & 20.56\end{array}$

$\begin{array}{llllll}17 & 1752 & 27.10 & 19 & 21.13\end{array}$ $\begin{array}{lllll}19 & 2336 & 53.72 & 20 & 39.29\end{array}$ 21 $020 \quad 44.12 \quad 19 \quad 19.45$ $\begin{array}{llllll}21 & 1850 & 2.77 & 19 & 14.66\end{array}$ 21 $1919 \quad 54.17 \quad 19 \quad 13.15$

$\begin{array}{llllll}22 & 013 & 48.74 & 19 & 19.95\end{array}$ $\begin{array}{llllll}22 & 22 & 3 & 25.68 & 19 & 3.36\end{array}$ $\begin{array}{lllll}23 & 147 & 58.56 & 19 & 19.16\end{array}$
LON W DEG MIN

15526.25

15512.02

1554.92

1556.68

1553.44

$155 \quad 35.87$

$155 \quad 5.78$

15618.96

15526.50

$155 \quad 13.42$

15513.46

1557.70

1552.10

15512.79

15512.80

$155 \quad 13.60$

15524.62

15525.80

1552.83

$156 \quad 1.43$

15515.26

15518.86

1559.80

1562.91

15546.74

1552.84

1556.01

1556.24

15511.02

15528.53

$155 \quad 6.67$

15525.16

$156 \quad 36.40$

$155 \quad 15.72$

15527.64

$155 \quad 16.77$

15524.99

1553.21

1553.49

1551.01

$155 \quad 1.22$

$155 \quad 47.03$

$155 \quad 13.35$

$156 \quad 13.07$

$156 \quad 20.48$

$155 \quad 6.83$

$156 \quad 39.01$

$155 \quad 15.46$

DEPTH AMP DUR

GAP RMS MIN ERH ERZ NO KM MAG MAG NR NS DEG SEC DIS KM KM FM REMK

$9.983 .2 \quad 3.222$

8.342 .73 .131

8.823 .13 .234

8.633 .33 .530

8. 122.83 .035

$10.92 \quad 3.0 \quad 3.3 \quad 33$

8.452 .83 .233

$32.36 \quad 3.0 ? .427$

10.192 .93 .136

$9.612 .7 \quad 3.233$

$10.25 \quad 3.6 \quad 3.934$

$8.84 \quad 2.7 \quad 3.0 \quad 34$

$7.48 \quad 3.3 \quad 3.837$

$36.492 .9 \quad 3.234$

$37.993 .0 \quad 3.134$

$9.542 .8 \quad 2.130$

8.103 .02 .919

$\begin{array}{llll}35.96 & 3.0 & 3.1 & 36\end{array}$

$8.39 \quad 2.7 \quad 3.021$

$9.813 .0 ? .422$

$10.18 \quad 3.1 \quad 3.3 \quad 37$

$6.42 \quad 2.6 \quad 3.234$

14.443 .43 .937

$\begin{array}{llll}7.89 & 2.9 & 3.0 & 23\end{array}$

16.013 .23 .536

9.032 .73 .034

$8.612 .7 \quad 3.0 \quad 33$

$6.252 .1 \quad 3.131$

$8.26 \quad 2.4 \quad 3.0 \quad 28$

9.132 .43 .128

$9.96 \quad 3.0 \quad 3.3 \quad 33$ $\begin{array}{llll}10.55 & 2.7 & 3.1 & 37\end{array}$ .053 .12 .641

$3.283 .3 \times .835$

$15.40 \quad 3.6 \quad 4.136$

2.212 .47 .131 $10.972 .9 \quad 3.236$ $6.08 \quad 3.4 \quad 4.0 \quad 37$

8.692 .83 .233

$8.923 . ? \quad 5.536$

8. 28 2.? 3.228

$6.953 .5 \quad 3.538$ $10.23 \quad 3.64 .038$

26.313 .94 .118

30.062 .3 .813

8.562 .72 .132

$7.003 .5 ? .815$ $10.514 .4 \quad 4.538$ $\begin{array}{llll}3 & 86 & .13 \quad 4\end{array}$

2 78.12

85.12

1128.09

2 110.09

1249.1126

080.136

2291.1145

91.136

167.105

$\begin{array}{llll}0 \quad 78 & .10 \quad 3\end{array}$

195.09

2174.11

60.093

060.103

273.123

2 77.13

1145.086

$\begin{array}{llll}0 & 197 \quad .15 \quad 20\end{array}$

$\begin{array}{llll}0 & 226.1120\end{array}$

$269.09 \quad 3$

$155.16 \quad 3$

$190 \quad .13 \quad 10$

$\begin{array}{llll}0 & 283 & .1422\end{array}$

$0 \quad 129.1114$

3130.12

1100.116

2. 115.146

092.095

06.1311
0.131

1117.095

$0 \quad 53.124$

8306.1285

144.123

0249.0923

2. $39.10 \quad 1$

136.14

1108.10

193.12

2204.10

$\begin{array}{llll}0 & 191.10 \quad 4\end{array}$

$\begin{array}{llll}6 & 218.20 & 50\end{array}$

371.105

$0320.1137 \quad 17^{\circ}$

$\begin{array}{lllll}0 & 327 & .08 & 50 & 24.7\end{array}$

0113.105

0113.10

0321.1385

2 91.10

.5

.6 $\begin{array}{llll}.5 & .8 & 19 & \text { UKF } \\ .4 & .8 & 23 & \text { UER } \\ .5 & .5 & 30 & \text { MER } \\ .5 & .6 & 29 & \text { UER } \\ .5 & .4 & 31 & \text { MER }\end{array}$

$1.5 \quad .729 \mathrm{KOH} \mathrm{F}$

.5 .730 MER

$3.0 \quad 1.625$ OIS

.4 .631 HEA

.4 .429 UER

.432 POL F

.531 UER

.537 MER

.71 .030 DEP

.71 .630 DEP

$.5 \quad .526$ POL

.51 .416 UKF

$.81 .235 \mathrm{LSW}$

$1.11 .819 \mathrm{HIL} B$

$1.5 .920 \mathrm{KON}$

$.4 .433 \mathrm{KOA}$

$.5 .931 \mathrm{KOA}$

$.5 .634 \mathrm{HIL} F$

$3.41 .019 \mathrm{KON}$

$1.1 \quad 1.635 \mathrm{KON} \mathrm{F}$

.4 .529 MER

.4 .530 UER

.51 .425 UER

.4 .628 UER

.5 .925 HEA

$.6 \quad .429$ UER

.4 .535 UKF

7.21 .834 DIS

$.3 \quad .531$ SPC F

$1.8 \quad 1.232$ DIS F

.4 .228 SPC F

.4 .532 UKF

.6 .532 MER F

$.7 \quad .528$ MER

433 MER

. 5 23 MER

$9 \quad 1.434$ DIS

.533 UER F

7.713 DIS

56 
ORIGIN TIME LAT N YEAR MON DA HRMN SEC DEG MIN

1978 JUN $27 \quad 2231 \quad 5.96 \quad 1924.78$

$\begin{array}{llllll}29 & 1831 & 10.89 & 19 & 21.75\end{array}$

$\begin{array}{llllll}30 & 1228 & 2.76 & 19 & 16.84\end{array}$

$\begin{array}{llllll}30 & 1420 & 34.92 & 19 & 19.63\end{array}$

$\begin{array}{llllll}30 & 2231 & 22.10 & 19 & 18.35\end{array}$

JUL

\begin{tabular}{|c|c|c|c|}
\hline 918 & 13.27 & 19 & \\
\hline 16 & 40.27 & 19 & \\
\hline 550 & 47.20 & 19 & \\
\hline 215 & 16.33 & 19 & \\
\hline 2254 & 31.03 & 19 & \\
\hline
\end{tabular}

$5 \quad 430 \quad 34.96 \quad 19 \quad 19.61$

$\begin{array}{lllll}7 & 1131 & 29.20 & 19 & 12.09\end{array}$

913756.911922 .28

$\begin{array}{llllll}13 & 8 & 3 & 48.97 & 19 & 13.30\end{array}$

$14 \quad 256 \quad 37.191921 .18$

142295.251917 .21

$\begin{array}{llllll}16 & 829 & 41.78 & 19 & 21.05\end{array}$

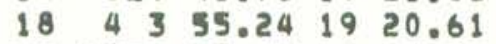

$22 \quad 1258 \quad 55.42 \quad 1921.02$

$23546 \quad 28.27 \quad 1927.08$

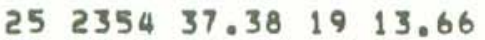

$\begin{array}{lllllll}27 & 10 & 9 & 26.55 & 19 & 29.03\end{array}$

AUG

$\begin{array}{llllll}1 & 12 & 8 & 36.49 & 19 & 19.90 \\ 4 & 1424 & 15.10 & 19 & 45.24 \\ 5 & 20 & 0 & 23.43 & 19 & 26.40\end{array}$

$\begin{array}{llll}5 & 2353.01 & 19 & 23.23\end{array}$

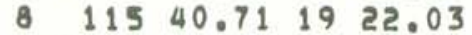

81116.251919 .86

$8110 \quad 10.28 \quad 1918.19$

944322.041927 .30

$11 \quad 144458.70 \quad 1941.03$

$\begin{array}{llllll}11 & 15 & 8 & 51.86 & 20 & 45.36\end{array}$

1205250.531920 .05

$\begin{array}{llllll}12 & 251 & 23.14 & 19 & 46.49\end{array}$

1214610.071918 .62

13224529.271920 .86

$\begin{array}{llllll}18 & 432 & 42.00 & 19 & 13.52\end{array}$

$\begin{array}{llll}18 & 2343 \quad 10.04 & 19 & 18.41\end{array}$

$19 \quad 212 \quad 29.11 \quad 1955.90$

$192018 \quad 14.78 \quad 1921.32$

$\begin{array}{llllll}21 & 2114 & 24.87 & 19 & 25.47\end{array}$

$\begin{array}{llllll}22 & 1111 & 27.17 & 19 & 19.12\end{array}$

$\begin{array}{llllll}23 & 1148 & 53.12 & 19 & 19.82\end{array}$

$\begin{array}{lllllll}23 & 1757 & 24.55 & 19 & 19.57\end{array}$

2321854.041923 .06

$\begin{array}{lllll}27 & 2046 \quad 59.87 & 19 & 22.91\end{array}$

$291144 \quad 8.82 \quad 19 \quad 19.05$

$\begin{array}{llllll}30 & 1240 & 15.66 & 19 & 20.45\end{array}$
LON W DEG MIN

15525.16

1556.99

15528.92

15510.35

15515.48

1556.73

$155 \quad 6.70$

15548.04

1554.86

$155 \quad 27.68$

1556.64

1567.13

$155 \quad 5.85$

$156 \quad 14.48$

15515.28

15512.66

1551.02

15510.70

1557.44

15452.65

1556.36

15538.98

$155 \quad 6.54$

1564.59

15523.99

$155 \quad 3.66$

1554.88

15511.94

15513.18

15449.44

1553.15

$155 \quad 59.54$

1556.84

156.25

15513.53

1557.33

15531.16

15513.35

15521.54

15525.70

15524.51

$155 \quad 7.70$

1558.16

15511.31

15516.78

15516.98

15511.78

$155 \quad 1.98$
DEPTH AMP DUR KM MAG MAG NR NS DEG SEC DIS KM

ERZ NO

$11.04 \quad 2.9 \quad 3.0 \quad 38$

9.233 .23 .337

$10.153 .3 \quad 5.737$

$9.873 .1 \quad 3.3 \quad 38$

$10.20 \quad 2.9 \quad 3.0 \quad 34$

7.974 .24 .323

$9.112 .8 \quad 3.0 \quad 33$

$12.35 \quad 2.9 \quad 3.1 \quad 38$

$9.07 \quad 3.4 \quad 3.6 \quad 37$

$11.08 \quad 3.0 \quad 3.5 \quad 37$

$7.43 \quad 2.8 \quad 3.4 \quad 37$

26.403 .13 .912

0.483 .13 .333

$26.86 \quad 3.12 .699$

$9.92 \quad 3.2 \quad 3.3 \quad 37$

$\begin{array}{rrrr}0 & 32 & .14 & 6 \\ 2 & 77 & .08 & 5 \\ 2 & 83 & .12 & 11 \\ 2 & 94 & .10 & 5\end{array}$

.4
.3
.4
.4
.7

KM FM REMK

.535 UKF

.534 UER

.532 HEA F

.533 UER

.530 KOA

$11.54 \quad 3.02 .938$

$7.84 \quad 3.4 \quad 3.7 \quad 34$

$9.29 \quad 2.7 \quad 3.134$

$8.10 \quad 2.7 \quad 3.0 \quad 31$

$9.103 .1 \quad 2.631$

.5

1.12 POL F

.429 UER

$.635 \mathrm{KON}$

.436 MER F

.532 UKF F

$51.453 .0 \quad 2.337$

$5.20 \quad 2.7 \quad 3.028$

$9.113 .0 \quad 3.4 \quad 34$

$30.873 .0 \quad 3.2 \quad 36$

$10.10 \quad 3.0 \quad 3.235$

$125.125 . .5$

.531 UER

$\begin{array}{lllll}0 & 322 & .12 & 27 & 19.5\end{array}$

7.712 DIS

.530 MER F

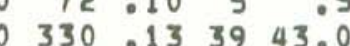

13.89 DIS *

2 68.09

.433 KOA F

$8.20 \quad 2.7 \quad 3.130$

$8.802 .7 ? .334$

$9.50 \quad 2.3 .034$

$10.44 \quad 3.7 \quad 4.0 \quad 36$

$8.623 .3 \quad 3.933$

$4.48 \quad 2.3 \quad 3.2 \quad 12$

15.953 .13 .631

$9.88 \quad 3.3 \quad 3.936$

11.392 .43 .529

9.312 .63 .132

8.472 .53 .132

$31.35 \quad 3.4 \quad 3.7 \quad 34$

$8.28 \quad 2.1 \quad 3.130$

$10.52 \quad 2.93 .134$

$8.88 \quad 2.0 \quad 3.0 \quad 33$

$10.97 \quad 3.0 \quad 3.0 \quad 37$

9.352 .23 .135

$8.393 .3 \quad 3.834$

$9.22 \quad 2.5 \quad 3.131$

3.692 .43 .130

$4.892 .9 \geqslant .334$

$9.953 .1 \mp .534$

7.033 .33 .629
1147.11

0196.11

176.12

284.12

1255.11

02.08 .09

072.15

1119.105

$038.12 \quad 26$
0

42.13

0101.11

178.10

184.11

94.09

\begin{tabular}{l}
277.14 \\
\hline
\end{tabular}

0242.1420

4323.1473

2110.095

3224.1120

2 84.10

188.115

068.069

$0 \quad 84.143$

$2189.13 \quad 20$
0.135

95.13

133.14

2 109.10

187.11

095.12

148.13

$0 \quad 104.09$

1197.10

$$
\begin{array}{rrrr}
.6 & .4 & 30 & \text { POL } \\
.9 & .5 & 33 & \text { MER F } \\
.4 & .5 & 30 & \text { UER } \\
.5 & .7 & 27 \text { UER } \\
1.6 & .4 & 26 \text { LER }
\end{array}
$$

$1.32 .232 \mathrm{POL}$

.51 .524 MOK F

$.6 \quad .428$ UER

$1.4 \quad 2.030 \mathrm{KON} \mathrm{F}$

.5 .734 UKF

$.5 \quad .624$ MER

. 530 MER

.529 UER

$\begin{array}{llll}.6 & .5 & 33 & \text { POL F } \\ 3.0 & .6 & 30 & \text { LER F }\end{array}$

$\begin{array}{rrrrr}.6 & .5 & 33 & \text { POL } F \\ 3.0 & .6 & 30 & \text { LER } & F\end{array}$

2.233 .48 HIL B*

15.444 .322 DIS *

.5 .432 UER F

1.0 .624 KON F

$.4 \quad .523$ POL
.5 .622 UER

$.6 \quad .830 \mathrm{POL}$

$.9 \quad .729 \mathrm{KKU}$

.5 .832 HEA

$.4 \quad .533$ UKF

.5 .4 30 UER

.5 . 52 UER

$.6 \quad .530$ UER

.3 .630 SPC

.4 .7 31 KOA

.5

.527 UER F .528 MER F
$.61 .333 \mathrm{LSW}$ 
ORIGIN TIME LAT N YEAR MON DA HRMN SEC DEG MIN

1978 AUG $30 \quad 1627 \quad 4.14 \quad 1921.46$ $\begin{array}{llllll}31 & 137 & 20.97 & 18 & 58.73\end{array}$ $\begin{array}{llll}31 & 1322 \quad 54.96 \quad 19 & 19.42\end{array}$ $\begin{array}{llllll}31 & 1414 & 53.51 & 18 & 59.56\end{array}$ SEP 14125.151920 .38 $\begin{array}{rrrrrr}2 & 17 & 5 & 4.00 & 19 & 32.46 \\ 3 & 426 & 10.51 & 19 & 18.84 \\ 4 & 2014 & 44.10 & 19 & 19.31 \\ 5 & 618 & 18.19 & 19 & 19.84\end{array}$ $5 \quad 1026 \quad 46.87 \quad 1920.13$

$\begin{array}{llll}5 \quad 1738 & 20.55 \quad 19 & 23.07\end{array}$

$7 \quad 44255.241914 .42$

$\begin{array}{lllll}7 & 1137 & 46.03 & 19 & 21.76\end{array}$

$\begin{array}{lllll}7 & 1656 & 53.39 & 19 & .21\end{array}$

$11 \quad 121 \quad 57.18 \quad 18 \quad 58.08$

$112016 \quad 6.11 \quad 1920.00$

$\begin{array}{lllll}13 & 1558 & 32.54 & 19 & 21.61\end{array}$

$132238 \quad 7.14 \quad 1924.86$

$\begin{array}{llllll}15 & 114 & 41.06 \quad 19 & 20.77\end{array}$

$1571558.3120 \quad 15.24$

$\begin{array}{lllll}15 & 723 & 55.65 & 19 & 25.33\end{array}$

$\begin{array}{lllll}16 & 630 & .61 & 19 & 23.97\end{array}$

$\begin{array}{llllll}16 & 1747 & 1.85 & 19 & 20.12\end{array}$

$\begin{array}{lllll}16 & 2141 & 5.36 & 19 & 22.17\end{array}$

$\begin{array}{lllll}17 & 351 & 46.60 & 19 & 21.77\end{array}$

$\begin{array}{lllll}18 & 2344 & 29.20 & 19 & 23.38\end{array}$

$\begin{array}{llll}19 & 843 & 44.06 \quad 19 \quad 2.59\end{array}$

$\begin{array}{lllll}19 & 1541 & 8.71 & 19 & 26.22\end{array}$

$\begin{array}{llllll}20 & 1028 & 56.03 & 19 & 18.91\end{array}$

$\begin{array}{lllll}20 & 1320 & 26.99 & 19 & 30.67\end{array}$

2121512.651953 .24

21 $2027 \quad 55.5919 \quad 19.37$

$\begin{array}{lllll}22 & 127 & .14 & 19 & 19.20\end{array}$

$\begin{array}{llllll}22 & 20 & 5 & 44.04 & 19 & 25.95\end{array}$

$241249 \quad 9.911922 .96$

$24 \quad 1530 \quad 53.02 \quad 19 \quad 23.36$

$\begin{array}{llllll}25 & 1330 & 13.54 & 19 & 19.51\end{array}$

$\begin{array}{lllllll}25 & 20 & 2 & 33.99 & 18 & 58.31\end{array}$

$\begin{array}{llllll}25 & 2335 & 31.51 & 19 & 20.11\end{array}$

$\begin{array}{llllll}26 & 1912 & 7.79 & 19 & 26.87\end{array}$

$\begin{array}{llllll}28 & 1538 & 20.19 & 19 & 22.99\end{array}$

$\begin{array}{lllll}30 & 1356 & 13.29 & 19 & 23.03\end{array}$

OCT

$\begin{array}{lllll}7 & 3 & 39.32 & 19 & 17.38 \\ 7 & 548 & 45.46 & 19 & 22.93\end{array}$

$\begin{array}{llllll}7 & 1811 & 7.54 & 19 & 12.67\end{array}$

$9 \quad 217 \quad 27.74 \quad 19 \quad 23.19$

$9855 \quad 9.39 \quad 19 \quad 13.58$

$\begin{array}{llll}10 & 1117 & 22.38 & 1957.42\end{array}$
LON W DEG MIN

$155 \quad 2.72$

15529.29

$155 \quad 12.56$

15528.67

$155 \quad 6.37$

$155 \quad 56.15$

15512.21

1558.49

1558.24

15513.60

15517.01

$156 \quad 11.51$

1554.05

15618.22

15520.62

1556.52

1552.53

15529.72

$155 \quad 6.60$

156.24

15516.50

15515.68

15511.68

1553.42

155.24

$155 \quad 16.77$

15541.46

15523.95

15511.56

15552.63

1557.58

15511.32

15510.74

$155 \quad 27.87$

15516.98

15525.04

1557.89

15520.90

15510.97

15522.75

15517.03

15526.70

15521.73

15517.10

15521.06

$155 \quad 17.01$

15540.25

15549.53

DEPTH AMP OUR

GAP RMS MIN ERH KM MAG MAG NR NS OEG SEC DIS KM

ERZ NO

$\begin{array}{lllll}7.60 & 2.7 & 3.1 & 32\end{array}$

$38.514 .3 \quad 4.6 \quad 36$

$9.94 \quad 2.5 \quad 3.4 \quad 32$

$37.25 \quad 3.2 \quad 3.6 \quad 37$

9.352 .73 .133

$10.792 .9 \quad 3.222$

$9.602 .7 \quad 3.4 \quad 38$

8.573 .13 .336

$9.563 .2 \times .637$

$10.09 \quad 3.7 \quad 4.2 \quad 34$

$\begin{array}{llll}3.63 & 2.7 & 3.3 & 33\end{array}$

16.763 .23 .323

$6.793 .2 \quad 3.7 \quad 33$

26.732 .93 .322

$53.32 \quad 3.64 .134$

$9.60 \quad 4.14 .3 \quad 37$

7.372 .93 .230

10.0 ?. $.9 \quad 3.0 \quad 34$

$8.38 \quad 2.73 .029$

16.493 .03 .031

$2.06 \quad 2.8 \quad 3.6 \quad 32$

$3.672 .8 \quad 3.235$

$9.162 .8 \times .134$

$6.72 \quad 2.6 \quad 3.130$

8.962 .63 .133

3.583 .23 .636

43.143 .12 .436

$9.223 .7 \% .540$

8.662 .93 .431

$9.633 .1 \% .330$

$42.98 \quad 3.1 \quad 3.2 \quad 37$

9.902 .73 .135

$9.613 .3 \quad 3.738$

$\begin{array}{llll}11.22 & 2.9 \quad 3.0 \quad 36\end{array}$

$2.812 .43 .0 \quad 28$

$10.66 \quad 2.4 \quad 3.0 \quad 35$

8. 863.13 .336

$17.32 \quad 3.2 \quad 0.537$

9.742 .23 .237

9.182 .07 .436

$3.312 .9 \quad 3.433$

9.443 .62 .938

$8.462 .53 .0 \quad 34$

$2.92 \quad 2.5 \quad 3.435$

$47.872 .3 \quad 3.228$

$2.872 .5 \quad 3.135$

$9.70 \quad 2.5 \quad 3.0 \quad 26$

$27.853 .1 \times .237$

58

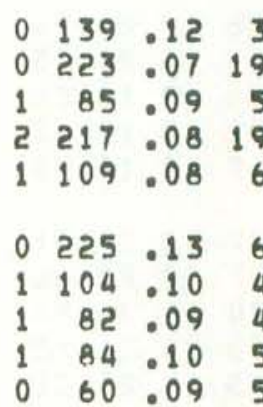

138.102 0266.1140 094.134 $\begin{array}{llll}0 & 291 & .09 & 54\end{array}$ 0234.0725

$\begin{array}{rrrr}1 & 117 & .10 & 6 \\ 1 & 144 & .09 & 3 \\ 2 & 54 & .10 & 6 \\ 0 & 98 & .09 & 6 \\ 1 & 307 & .20 & 27\end{array}$

1

$37.10 \quad 1$

160.113

$\begin{array}{llll}0 & 81.08 \quad 5\end{array}$

$0112.10 \quad 4$

0192.106

$\begin{array}{llll}0 & 38 & .10 \quad 2\end{array}$ 2165.08

2 36.12

$0 \quad 110.10$

$\begin{array}{lll}0 & 129.17\end{array}$

$2240 \quad .10 \quad 19$

099.096

1105.126

$0 \quad 34.136$

048.092

$\begin{array}{llll}0 & 58.115\end{array}$

198.094

1232.2333

184.104

141.116

148.10

154.13

1125.136

$0 \quad 37.101$

2 164.0811

136.100

$\begin{array}{llll}0 & 237 & .23 & 6\end{array}$

0183.0820

$\begin{array}{rrrl}.7 & .5 & 29 & \text { MER } \\ 1.3 & 2.1 & 32 & \text { DIS F } \\ .5 & .4 & 29 & \text { UER } \\ 1.0 & 1.5 & 32 & \text { DIS } \\ .5 & .5 & 30 & \text { UER }\end{array}$

$1.3 .517 \mathrm{KON}$

.4 .433 POL F

.5

.5

.434 UER

. 435 UER

. 430 UER F

.3 .629 SPC F

3.384 .021 DIS

.5 .730 MER

6.34 .120 DIS

$1.62 .632 \mathrm{PPL}$

.6 .433 UER F

.6 .525 MER

.4 .628 UKF

$.5 \quad .629$ UER

4.940 .926 DIS

.3 .231 SPC F

.4 .533 SPC F

$.4 \quad .429$ UER

$.4 \quad .629$ MER

.8 .430 LER

.3

.533 SPC F

2. 034 HEA

.937 UKF F

.528 POL F

$.526 \mathrm{KON}$

$1.2 \quad 1.6 \quad 31 \mathrm{KKU}$

.5 .433 UER

.5 .432 UER F

.4 .633 UKF

.3 .527 KOA

.4 .433 UKF

$.5 \quad .433$ UER

$2.131 .835 \mathrm{PPL}$

.4 .434 UER

.4 .733 UKF

.3 .532 KOA F

.4 .738 UKF F

$.5 \quad .730$ SWR

$.3 .427 \mathrm{KOA}$

$1.01 .919 \mathrm{LSW} \mathrm{L}$

.3 .331 SPC

2.21 .122 HEA $1.33 .035 \mathrm{KOH}$ 

$\begin{array}{lll}\text { ORIGIN TIME } & \text { LAT } N \\ \text { YEAR MON DA HRMN SEC DEG MIN }\end{array}$ $\begin{array}{lllllll}1978 & \text { OCT } 11 \quad 734 & 58.48 & 19 & 13.11\end{array}$ $\begin{array}{llllll}12 & 2227 & 36.90 & 19 & 20.56\end{array}$ $\begin{array}{lllll}13 & 1230 & 17.56 & 19 & 13.54\end{array}$ $131240 \quad 19.301915 .26$ $131250 \quad 16.091915 .43$

$\begin{array}{llllll}13 & 13 & 0 & 21.62 & 19 & 14.97\end{array}$ $13131018.84 \quad 19 \quad 15.44$ $\begin{array}{llllll}13 & 1320 & 20.56 & 19 & 16.92\end{array}$

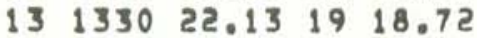
1553548.651922 .93

$\begin{array}{lllll}17 & 516 & 24.23 & 19 & 23.28\end{array}$ 17224244.651919 .89 $18 \quad 616 \quad 55.55 \quad 1921.32$ $\begin{array}{lllll}20 & 239 & 53.77 & 19 & 26.37\end{array}$ $\begin{array}{llllll}22 & 1513 & 1.48 & 19 & 12.47\end{array}$

$24 \quad 1433 \quad 13.99 \quad 19 \quad 19.47$ $\begin{array}{llllll}28 & 1237 & 34.70 & 21 & 34.97\end{array}$ $\begin{array}{llllll}28 & 1644 & 13.37 & 19 & 12.08\end{array}$ $2905128.19 \quad 1925.18$ $\begin{array}{lllll}31 & 2312 & 1.42 & 19 & 20.23\end{array}$

$145757.8319 \quad 19.33$

$3346 \quad 53.851927 .69$

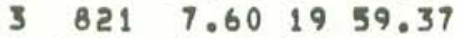

$\begin{array}{lllll}5 & 1625 & 1.60 & 19 & 19.67\end{array}$

604816.661919 .50

$\begin{array}{lllll}7 & 1256 & 57.79 & 19 & 48.04\end{array}$

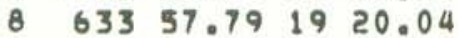

$\begin{array}{llllll}8 & 2146 & 39.00 \quad 19 & 20.73\end{array}$

$\begin{array}{llllll}10 & 20 & 5 & 23.36 & 19 & 21.80\end{array}$

121582.471922 .08

$\begin{array}{llllll}12 & 1526 & 5.15 & 19 & 22.39\end{array}$

$\begin{array}{llllll}13 & 232 & 12.93 & 19 & 19.17\end{array}$

$\begin{array}{lllll}15 & 337 & 17.74 .19 & 25.43\end{array}$

$15105318.98 \quad 19 \quad 8.40$

15132929.951920 .09

$151649 \quad 46.751924 .24$ $17 \quad 1124 \quad 40.40 \quad 1940.67$

$1983520.17 \quad 1920.04$

$\begin{array}{llllll}22 & 526 & 36.46 & 19 & 22.40\end{array}$

$\begin{array}{llllll}22 & 820 & 42.87 & 19 & 12.68\end{array}$

$\begin{array}{llllll}22 & 1323 & 14.81 & 19 & 20.97\end{array}$

$\begin{array}{llllll}23 & 117 & 36.15 & 19 & 21.90\end{array}$

$23 \quad 316 \quad 15.88 \quad 19 \quad 14.16$

$\begin{array}{llllll}24 & 148 & 8.43 & 19 & 21.12\end{array}$

$\begin{array}{llll}2510 & 2 & 8.9419 & 190.07\end{array}$

$26 \quad 12 \quad 147.91 \quad 1923.04$

$27161950.1419 \quad 20.44$

$\begin{array}{llllll}28 & 131 & 36.75 & 19 & 18.74\end{array}$
LON W DEG MIN

$155 \quad 34.47$

15513.13

$156 \quad 39.01$

15639.21

$156 \quad 39.21$

$\begin{array}{lll}156 & 18.74\end{array}$

$156 \quad 20.29$

$156 \quad 14.22$

$156 \quad 12.91$

1554.32

15516.86

1557.80

$155 \quad 15.35$

$155 \quad 23.99$

15519.93

15512.04

$157 \quad 44.49$

15520.81

$155 \quad 15.99$

$155 \quad 6.39$

15511.55

15534.81

1558.71

15511.20

1558.84

$156 \quad 8.42$

1558.43

1558.64

$155 \quad 4.99$

1554.94

1556.20

13514.13

15524.31

$155 \quad 36.33$

1557.44

$155 \quad 24.74$

$155 \quad 2.40$

$155 \quad 5.14$

1554.05

$155 \quad 27.98$

$155 \quad 3.13$

$155 \quad 6.67$

15532.81

$155 \quad 13.30$

1558.40

1556.23

15511.69

$155 \quad 13.19$

DEPTH AMP DUR

GAP RMS MIN ERH

ERZ NO KM MAG MAG NR NS DEG SEC DIS KM KM FM REMK

$3.52 \quad 3.2 \quad 3.3 \quad 35$

$9.27 \quad 2.4 \quad 3.134$

.103 .23 .213

$.103 . ? 2.812$

.103 .23 .213

.103 .03 .115

$.103 .0 \quad 3.016$

$.10 \quad 2.9 \quad 3.0 \quad 17$

$.102 .5 \quad 3.222$

8.413 .64 .036

$3.18 \quad 2.9 \quad 3.7 \quad 33$

9.573 .23 .736

9.50 दे. 33.036

$8.80 \quad 3.3 \quad 3.4 \quad 36$

46.842 .93 .240

$9.862 .9 \quad 3.336$

$1.22 \quad 4.14 .538$

$48.40 \quad 3.2 \quad 3.735$

$1.97 \quad 2.5 \quad 3.232$

9.672 .83 .234

10.002 .93 .536

$\begin{array}{lllll}37.31 & 2.7 & 3.3 & 24\end{array}$

$6.662 .13 .1 \quad 19$

8.993 .13 .335

$7.932 .6 \quad 3.0 \quad 12$

$12.35 \quad 3.0 \quad 2.9 \quad 17$

$8.75 \quad 2.8 \quad 3.0 \quad 22$

B. 18 2.R 3.326

9.043 .33 .730

.003 .23 .723

$1.18 \quad 2.7 \quad 3.1 \quad 18$

$\begin{array}{llll}8.78 & 2.9 & 3.2 & 28\end{array}$

$9.86 \quad 3.2 \quad 3.434$

6.332 .03 .125

8.872 .63 .130

$11.34 \quad 3.3 \quad 3.4 \quad 37$

$10.16 \quad 2.9 \quad 3.2 \quad 23$

$9.992 .6 \quad 3.4 \quad 19$

$9.532 .6 \quad 3.5 \quad 34$

$5.243 .1 \quad 3.431$

$6.06 \quad 3.4 \quad 3.8 \quad 30$

9.252 .63 .232

$11.404 . ? 4.233$

$9.022 .6 \quad 3.033$

9.772 .13 .231

.592 .43 .110

$10.36 \quad 3.3 \quad 3.6 \quad 35$

$10.133 .6 \quad 3.9 \quad 37$

59

$\begin{array}{llllllllll}1 & 81 & .23 & 13 & .7 & 3.2 & 29 & \text { HEA * }\end{array}$

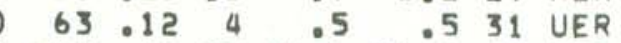

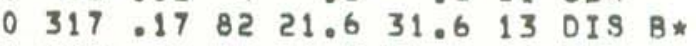

$\begin{array}{lllllll}0 & 325.20 & 81 & 34.8 & 31.6 & 11 & \text { DIS } B *\end{array}$

$\begin{array}{llllllll}0 & 325.25 & 81 & 35.7 & 31.6 & 13 & \text { DIS } & \text { B* }\end{array}$

$\begin{array}{llll}0 & 276 & .31 & 46\end{array}$

278.3949
0.397

$\begin{array}{llll}0 & 267.29 & 41\end{array}$

0264.4336

189.093

138.113

95.115

166.092

144.147

$3169.12 \quad 10$

$191.10 \quad 5$

$\begin{array}{lllll}6 & 242 & 30 & 33\end{array}$

$\begin{array}{llll}0 & 168.10 \quad 12\end{array}$

140.102

$0 \quad 113.106$

0
1

134.093

$\begin{array}{llll}0 & 282 & .2123\end{array}$

193.115

0207.145

1200.0934

1199.105

1170.11

179.115

1150.25$$
68.10
$$$$
139.128
$$$$
0 \quad 118.17 \quad 15
$$$$
99.09
$$

$131.12 \quad 6$

$\begin{array}{llll}0 & 197 \quad .17 & 19\end{array}$

$1132.12 \quad 4$

96.074

0101.156

1121.12 2

077.10

0122.09

57.10

80.09

94.10

176.08

84.10
5.431 .613 DIS B* 6.331 .615 DIS $B$ * 4.131 .617 DIS B* 5.131 .621 DIS $B$ * .5 .435 MER F

$.3 \quad .7 \quad 30 \quad S P C$

$.6 \quad .434$ UER

.4 .432 KOA

.4 .931 UKF

.81 .338 HLP

.4 .434 UER

6.31 .827 DIS F*

1.02 .231 HLP

.2 .328 SPC F

.6 .433 UER

.5 .4 35 UER

.71 .718 MOK

$6.21 .614 \mathrm{KKU}$

.5 .6 31 UER

1.91 .010 UER

$1.7 .915 \mathrm{KON}$

.9

.717 UER

.623 UER

.627 MER

$1.0 \quad 1.2$ 20 MER

.61 .014 UER

.5 .626 UER F

$.4 \quad .630$ UKF

.71 .321 HEA

$.5 \quad .6 \quad 27$ UER

.4 .534 UKF

$1.11 .322 \mathrm{HIL} B$

.9 .515 MER

$.5 \quad .430$ MER

$.61 .628 \mathrm{LSW}$

$.7 \quad .927$ MER

$.5 \quad .528$ UER

.5

$.633 \mathrm{LSWF}$

.628 UER

.529 UER

.62 .110 GLN

.4 .430 UER

$.5 .436 \mathrm{POL}$ 
HVO EARTHQUAKE SUMMARY LIST $(M>3)$

PAGE 7

ORIGIN TIME LAT N DA HRMN SEC

YEAR MON

$1978 \mathrm{NOV}$

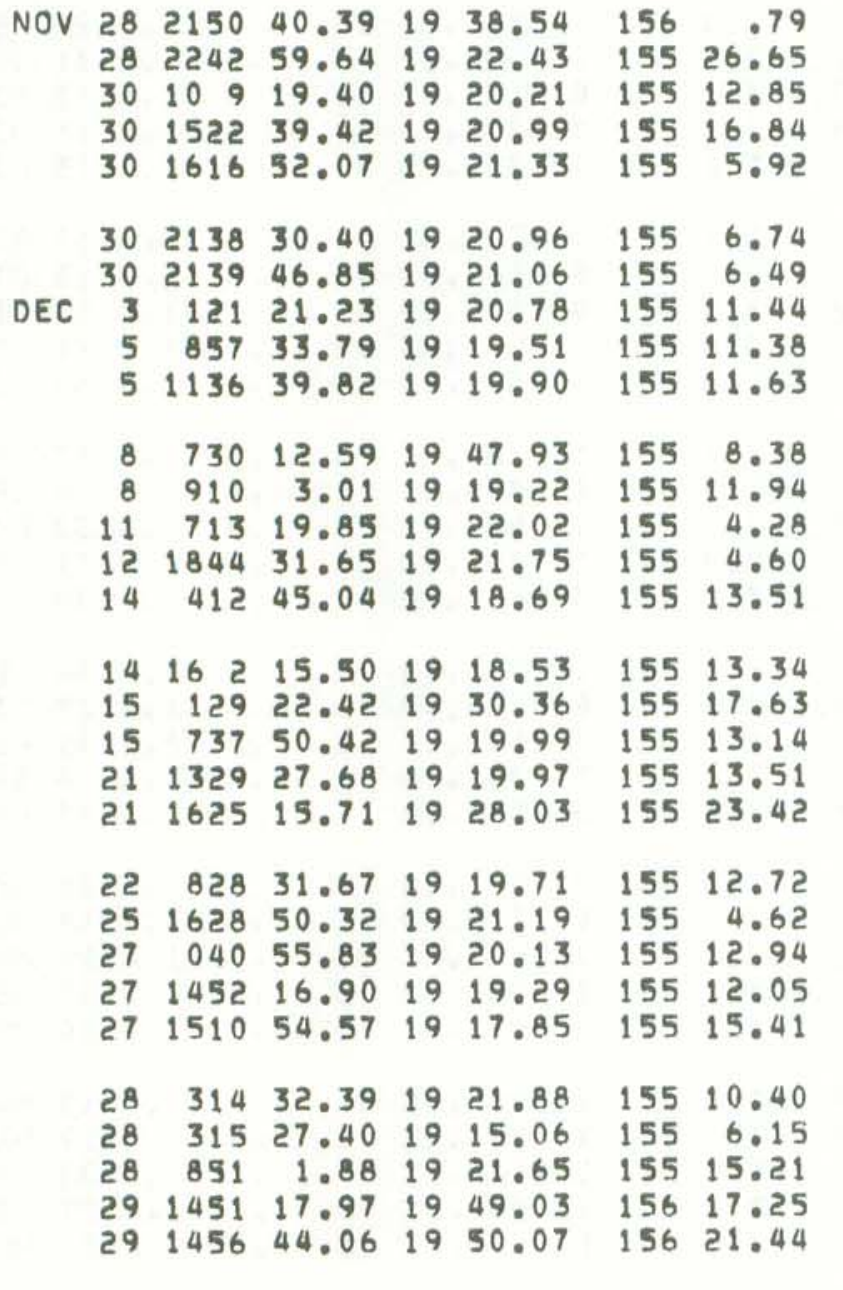

$\begin{array}{lllllll}31 & 248 & 39.41 & 19 & 31.92 & 155 & 41.90\end{array}$
DEPTH AMP NIIR GAP RMS MIN ERH KM MAG MAG NR NS DEG SEC DIS KM

$\begin{array}{lllllllll}10.68 & 3.3 & 3.4 & 34 & 2 & 224 & .11 & 20 & 1.1\end{array}$

$11.133 .5 \quad 3.635$

$10.352 .7 \quad 2.3 \quad 34$

$29.47 \quad 3 . ? 3.5 \quad 37$

9.723 .33 .635

$8.77 \quad 2.7 \quad 3.0 \quad 31$

7.013 .03 .233

$9.88 \quad 3.0 \quad 3.3 \quad 34$

$9.38 \quad 2.9 \quad 3.2 \quad 37$

9.743 .24 .037

41.452 .93 .241

$9.793 .2 \times .435$

9.442 .93 .233

$8.72 \quad 3.94 .237$

$10.49 \quad 4.14 .237$

$10.29 \quad 2.9 \quad 3.230$

$11.18 \quad 2.4 \quad 3.135$

$10.073 .3 \quad 3.636$

$10.20 \quad 3.5 \quad 3.935$

$8.493 .3 \times .135$

$9.272 .6 \quad 3.129$

$8.983 .2 \quad 3.434$

9.934 .74 .635

9.602 .03 .234

8.602 .63 .132

$33.773 .9 \quad 3.236$

$47.52 \quad 3.4 \quad 3.8 \quad 36$

$9.413 .4 \quad 3.434$

$6.36 \quad 3.4 \times .928$

$\begin{array}{llll}2.29 & 2.7 \quad 3.129\end{array}$

$9.483 .0 \geq .932$
ERZ NO

KM FM REMK

$.833 \mathrm{KON} F$ .534 UKF F .329 UER 1.130 DEP .331 MER

.629 UER .828 UER . $\triangle 32$ UER .427 UER .525 UER

$193.11 \quad 10$
98

$\begin{array}{llll}.4 & .5 & 21 & \text { UER } \\ .6 & .4 & 32 & \text { MER } \\ .5 & .4 & 34 & \text { UER } \\ .5 & .4 & 28 & \text { UER } \\ .6 & .7 & 32 & \text { KOA }\end{array}$
$98.10 \quad 5$

$89.08 \quad 5$

81.09

74.10

83.10

57.11

68.09

62.11

77.15

77.153

78.105

92.11

70.08

95.12

142.10

.5

6

.6

.5

$\because 4$

$.5 \quad .429 \mathrm{POL}$

.4 .630 GLN

.5

.5 . 430 UER .435 UER .835 UKF

.71 .230 UER $.91 .630 \mathrm{POL}$ $.4 .528 \mathrm{KOA}$ $\begin{array}{rrrrrrrr}0 & 322 & .11 & 49 & 15.7 & 5.5 & 26 & \text { DIS } \\ 0 & 322 & .11 & 57 & 11.2 & 11.4 & 22 & \text { DIS }\end{array}$ $\begin{array}{llllllll}62 & .12 & 7 & .5 & .7 & 28 & & \end{array}$ 